Um esquema "upwind" para leis de conservação e sua aplicação na simulação de escoamentos incompressíveis 2D e 3D laminares e turbulentos com superfícies livres 

SERVIÇO DE PÓS-GRADUAÇÃO DO ICMC-USP

Data de Depósito:

Assinatura:

\title{
Um esquema "upwind" para leis de conservação e sua aplicação na simulação de escoamentos incompressíveis 2D e 3D laminares e turbulentos com superfícies livres ${ }^{1}$
}

\author{
Fernando Akira Kurokawa
}

\section{Orientador: Prof. Dr. Valdemir Garcia Ferreira}

Co-orientador: Prof. Dr. Murilo Francisco Tomé

Tese apresentada ao Instituto de Ciências Matemáticas e de Computação - ICMC-USP, como parte dos requisitos para obtenção do título de Doutor em Ciências - Ciências de Computação e Matemática Computacional.

\section{USP - São Carlos}

Fevereiro de 2009

\footnotetext{
${ }^{1}$ Projeto financiado pela FAPESP $(n \bullet 05 / 51458-0)$
} 

Dedico às pessoas que mais amo na vida:

minha família. 


\section{Agradecimentos}

A minha família que sempre está presente em minha vida.

Ao professor Valdemir Garcia Ferreira, pela orientação, apoio e disponibilidade ao longo destes anos.

Aos professores do LCAD: Poti, Murilo, Castelo, Fabrício, Leandro, Nonato e Buscaglia.

Aos professores Fernando Tavares Pinho e Manuel Alves, da Universidade do Porto, e ao professor Miguel Nóbrega, da Universidade do Minho, pelo suporte e acolhimento durante a minha estadia em Portugal.

A professora Eliana X. L. de Andrade e ao professor Alagacone Sri Ranga pela amizade, apoio e incentivo desde o período da graduação.

Aos meus amigos, amigas e colegas que acompanharam as alegrias e dificuldades neste período, sempre me apoiando.

Aos funcionários do ICMC que sempre foram prestativos ao longo destes anos.

A todos que direta ou indiretamente, contribuíram para o sucesso deste trabalho.

À FAPESP, pelo apoio financeiro no desenvolvimento deste trabalho.

Finalmente, e acima de tudo, a Deus por estar presente em todos os momentos da minha vida. 


\section{Resumo}

Apesar de as EDPs que modelam leis de conservação e problemas em dinâmica dos fluidos serem bem estabelecidas, suas soluções numéricas continuam ainda desafiadoras. Em particular, há dois desafios associados à computação e ao entendimento desses problemas: um deles é a formação de descontinuidades (choques) e o outro é o fenômeno turbulência. Ambos os desafios podem ser atribuídos ao tratamento dos termos advectivos não lineares nessas equações de transporte. Dentro deste cenário, esta tese apresenta o estudo do desenvolvimento de um novo esquema "upwind" de alta resolução e sua associação com modelagem da turbulência. O desempenho do esquema é investigado nas soluções da equação de advecção 1D com dados iniciais descontínuos e de problemas de Riemann 1D para as equações de Burgers, Euler e águas rasas. Além disso, são apresentados resultados numéricos de escoamentos incompressíveis 2D e 3D no regime laminar a altos números de Reynolds. O novo esquema é então associado à modelagem $\kappa-\varepsilon$ da turbulência para a simulação numérica de escoamentos incompressíveis turbulentos 2D e 3D com superfícies livres móveis. Aplicação, verificação e validação dos métodos numéricos são também fornecidas.

Palavras-chave: Esquema "upwind" de alta resolução, solução numérica de leis de conservação, método de diferenças finitas, modelagem de $\kappa-\varepsilon$ da turbulência, escoamentos com superfícies livres, equações de Navier-Stokes, equações médias de Reynolds. 


\section{Abstract}

Although the PDEs that model conservation laws and fluid dynamics problems are well established, their numerical solutions have presented a continuing challenge. In particular, there are two challenges associated with the computation and the understanding of these problems, namely, formation of shocks and turbulence. Both challenges can be attributed to the nonlinear advection terms of these transport equations. In this scenario, this thesis presents the study of the development of a new high-resolution upwind scheme and its association with turbulence modelling. The performance of the scheme is investigated by solving the 1D advection equation with discontinuous initial data and 1D Riemann problems for Burgers, Euler and shallow water equations. Besides, numerical results for 2D and 3D incompressible laminar flows at high Reynolds number are presented.

The new scheme is then associated with the $\kappa-\varepsilon$ turbulence model for the simulation of $2 \mathrm{D}$ and $3 \mathrm{D}$ incompressible turbulent flows with moving free surfaces. Application, verification and validation of the numerical methods are also provided.

Key words: High-resolution upwind scheme, conservation laws, numerical simulation, finite difference method, turbulence modelling, free surface flows, Navier-Stokes equations, averaged Navier-Stokes equations. 



\section{Lista de Figuras}

2.1 A decomposição de Reynolds para o campo de velocidade $u(\mathbf{x}, t)$.

2.2 Regiões diferentes de um escoamento próximo à uma parede. Adaptado de MacDonough (2004). . . . . . . . . . . . . . . . . . . 26

3.1 Posições dos nós $\mathbf{D}$, U e $\mathbf{R}$ conforme o sinal da variável convectada $\phi$ na face $f$,

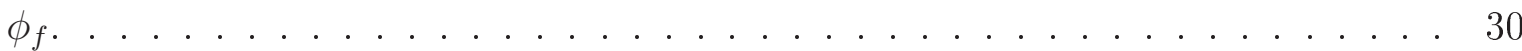

3.2 Representação esquemática para se obter aproximações dos termos advectivos, mostrando: $\mathrm{O}$ ponto $\mathcal{P}$ de discretização e seus vizinhos, as faces envolvidas $f$ e $g$ para a aproximação e a direção das velocidades $\mathbf{V}_{\mathbf{f}}$ e $\mathbf{V}_{\mathbf{g}}$ de advecção nas faces

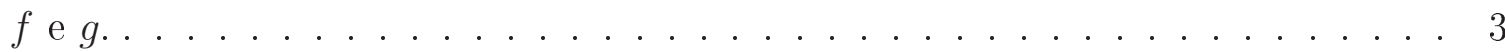

3.3 Representação esquemática do comportamento monotônico local da variável ge-

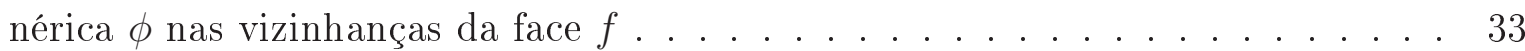

3.4 Região CBC no plano $\hat{\phi}_{f}-\hat{\phi}_{U} \ldots \ldots \ldots \ldots \ldots \ldots$

3.5 Comparação entre a solução numérica obtida com o esquema Upwind (símbolo azul) e a solução exata (linha vermelha), para a equação de transporte de um escalar. . . . . . . . . . . . . . . . . . . 36

3.6 Comparação entre a solução numérica obtida com o esquema Lax-Wendroff (símbolo azul) e a solução exata (linha vermelha), para a equação de transporte de um escalar.

3.7 Comparação entre a solução numérica obtida com o esquema QUICKEST (símbolo azul) e a solução exata (linha vermelha), para a equação de transporte de um escalar. . . . . . . . . . . . . . . . . . . . . . . 37

3.8 Região TVD de Sweby. . . . . . . . . . . . . . . . . . . . . . 38

3.9 Gráfico da variável normalizada de $\hat{u}_{f}$ para o esquema QUICKEST (vermelho) e o esquema ADBQUICKEST $\operatorname{com} \theta=0.5$ (azul) . . . . . . . . . . . . 39

3.10 Limitador de fluxo do esquema ADBQUICKEST na região TVD de Sweby para vários valores do número de Courant $\theta \ldots \ldots \ldots$. . . . . . . . . 42 
4.1 Célula computacional típica que mostra onde as variáveis são discretizadas. A variável genérica $\phi$ representa $p_{e}, \kappa, \varepsilon$ ou $\nu_{t} \ldots \ldots \ldots \ldots$. . . . . . . 44

4.2 Tipos de células no domínio computacional. . . . . . . . . . . . . . . . . . 45

4.3 Exemplo de uma superfície planar paralela ao plano formado pelos eixos $x y$ com

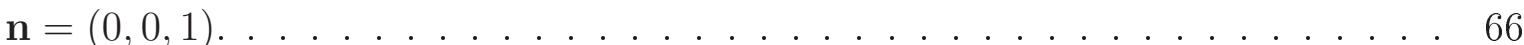

4.4 Exemplo de uma superfície planar inclinada de $45^{\circ} \operatorname{com} \mathbf{n}=\left(\frac{\sqrt{2}}{2}, 0, \frac{\sqrt{2}}{2}\right) \ldots$. . . 68

4.5 Exemplo de uma superfície planar inclinada de $60^{\circ}$ com $\mathbf{n}=\left(\frac{\sqrt{3}}{3},-\frac{\sqrt{3}}{3}, \frac{\sqrt{3}}{3}\right)$. . . 70

4.6 Representação esquemática de células adjacentes ao contorno rígido, mostrando a camada interna adjacente às paredes e as células fictícias. . . . . . . . . . . . . 72

5.1 Soluções exata e numérica da equação linear de advecção usando as condições iniciais (5.1) $\operatorname{com} \theta=0.3$.

5.2 Soluções exata e numérica da equação linear de advecção usando as condições iniciais (5.1) $\operatorname{com} \theta=0.8$.

5.3 Erro na solução numérica da equação linear de advecção (2.1) com a condição inicial (5.1) . . . . . . . . . . . . . . . . . . . . . .

5.4 Soluções exata e numérica da equação linear de advecção usando as condições iniciais (5.2) $\operatorname{com} \theta=0.3$.

5.5 Soluções exata e numérica da equação linear de advecção usando as condições iniciais (5.2) $\operatorname{com} \theta=0.8$.

5.6 Soluções exata e numérica da equação linear de advecção usando as condições iniciais (5.3) $\operatorname{com} \theta=0.3$.

5.7 Soluções exata e numérica da equação linear de advecção usando as condições iniciais (5.3) $\operatorname{com} \theta=0.8$.

5.8 Resultados numéricos para a equação de Burgers com as condições (5.5) e (5.4)

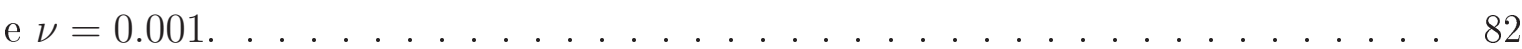

5.9 Resultados numéricos para a equação de Burgers com as condições (5.6)-(5.7). . 84

5.10 Soluções de referência e numérica para a profundidade $h$ no problema de águas

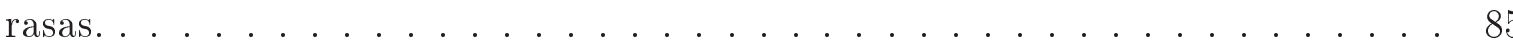

5.11 Soluções de referência e numérica da quantidade $h u$ para o problema de águas rasas. . . . . . . . . . . . . . . . . . . . 85

5.12 Perfil da densidade do problema teste $9 \operatorname{com} \theta=0.2 \ldots$. . . . . . . . . . . 86

5.13 Perfil da densidade do problema teste $9 \operatorname{com} \theta=0.6 \ldots$. . . . . . . . . . 86

5.14 Perfil da densidade do problema teste $10 \operatorname{com} \theta=0.2 \ldots \ldots$. . . . . . . 87

5.15 Perfil da densidade do problema teste $10 \operatorname{com} \theta=0.6 \ldots \ldots$. . . . . . . . 87

5.16 Perfil da densidade do problema teste $11 \operatorname{com} \theta=0.2 \ldots \ldots$. . . . . . . 88

5.17 Perfil da densidade do problema teste $11 \operatorname{com} \theta=0.6 \ldots \ldots$. . . . . . . . 88

5.18 Perfil da densidade do problema teste $12 \operatorname{com} \theta=0.2 \ldots \ldots$. . . . . . 89 
5.19 Perfil da densidade do problema teste $12 \operatorname{com} \theta=0.6 \ldots$. . . . . . . . . 89

5.20 Ampliação das soluções numérica e de referência da Figura 5.18 na região [1.6, 2.7]. 90

5.21 Ampliação das soluções numérica e de referência da Figura 5.19 na região [1.6, 2.7]. 90

5.22 Perfil da densidade do problema teste $13 \operatorname{com} \theta=0.2$ e 12500 células. . . . . . . 91

5.23 Perfil da densidade do problema teste $13 \operatorname{com} \theta=0.2$ e 18500 células. . . . . . . 91

5.24 Ampliação das soluções numérica e de referência da Figura 5.22 na região $[-2.2,1.2]$. 92

5.25 Ampliação das soluções numérica e de referência da Figura 5.22 na região [2.0, 3.2]. 92

5.26 Ampliação das soluções numérica e de referência da Figura 5.23 na região $[-2.2,1.2]$. 93

5.27 Ampliação das soluções numérica e de referência da Figura 5.23 na região [2.0, 3.2]. 93

5.28 Perfil da densidade do problema teste $13 \operatorname{com} \theta=0.6$ e 12500 células. . . . . . . 94

5.29 Perfil da densidade do problema teste $13 \operatorname{com} \theta=0.6$ e 18500 células. . . . . . . 94

5.30 Ampliação das soluções numérica e de referência da Figura 5.28 na região $[-0.5,0.5] .95$

5.31 Ampliação das soluções numérica e de referência da Figura 5.28 na região [2.0, 3.2]. 95

5.32 Ampliação das soluções numérica e de referência da Figura 5.29 na região $[-0.5,0.5]$. 96

5.33 Ampliação das soluções numérica e de referência da Figura 5.29 na região [2.0,3.2]. 96

5.34 Geometria do problema da expansão brusca laminar. . . . . . . . . . . . . . . 99

5.35 Problema da expansão brusca sem modelagem de turbulência para $R e=100$. . 100

5.36 Problema da expansão brusca sem modelagem de turbulenta para $R e=400$. . 100

5.37 Problema da expansão brusca sem modelagem de turbulenta para $R e=800$. . . 101

5.38 Comparação dos resultados experimentais e numéricos usando o esquema ADBQUICKEST. . . . . . . . . . . . . . . . . . . . 102

5.39 Perfis da componente $u$ da velocidade do problema da expansão brusca, mostrando a convergência da solução numérica nas quatro malhas usando o esquema ADBQUICKEST. . . . . . . . . . . . . . . . . 103

5.40 Ilustração da superfície livre de um jato de fluido incidindo sobre uma superfície rígida. . . . . . . . . . . . . . . . . . . . 103

5.41 Domínio do problema do jato livre sobre uma superfície rígida. . . . . . . . . . 105

5.42 Evolução do perfil da superfície livre e contorno da pressão para o problema do

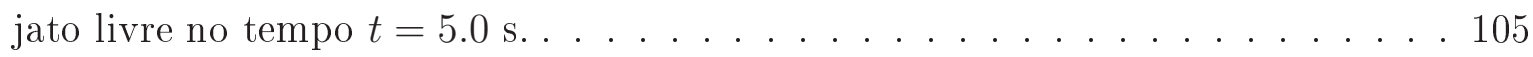

5.43 Evolução do perfil da superfície livre e contorno da velocidade na direção $x$ para

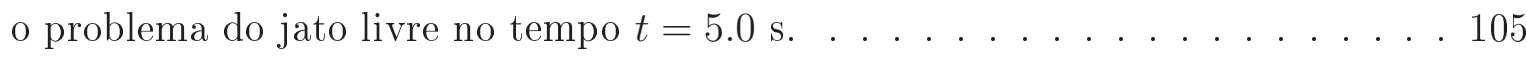

5.44 Comparação entre as soluções numéricas nas quatro malhas utilizando o esquema ADBQUICKEST e a solução analítica de Watson. . . . . . . . . . . . . . 106

5.45 Ampliação das soluções numéricas e da solução analítica de Watson. . . . . . . . 106

5.46 Diagrama esquemático do problema "Broken Dam". . . . . . . . . . . . . . 107

5.47 Comparação entre as soluções numéricas 2D nas três malhas e os dados experimentais para a posição frontal do movimento do fluido $\left(X_{\max }\right) \ldots$. . . . . . . 108 
5.48 Comparação entre os resultados numéricos 2D e 3D com os dados experimentais para a posição frontal do movimento do fluido $\left(X_{\max }\right)$. . . . . . . . . . . . . 109

5.49 Simulação numérica 3D utilizando o esquema ADBQUICKEST ilustrando a evolução da superfície livre em diferentes tempos.

5.50 Simulação numérica 2D do colapso da coluna de fluido utilizando o esquema ADBQUICKEST com $R e=99.045,44$.

5.51 Simulação numérica 3D do colapso da coluna de fluido utilizando o esquema ADBQUICKEST com $R e=99.045,44$.

5.52 Visualização de um exemplo muito comum de ressalto hidráulico circular.

5.53 Visualização da estrutura do ressalto hidráulico circular a número de Reynolds:(a) moderado; (b) elevado. . . . . . . . . . . . . . . . . . . . . . 114

5.54 Simulação numérica 3D obtida pelo código Freeflow 3D utilizando-se o esquema ADBQUICKEST, ilustrando o ressalto hidráulico circular em $t=2.7 \mathrm{~s}$. . . . . . 114

5.55 Comparação entre a solução numérica obtida pelo esquema ADBQUICKEST, e o experimento realizado por Taylor com $R e=200$ mostrando: (a) a simulação numérica 3D no tempo $t=40 \mathrm{~s}$; (b) a visualização do experimento de Taylor no tempo $t=40 \mathrm{~s}$; (c) simulação numérica 3D no tempo $t=100 \mathrm{~s}$; e (d) a visualização do experimento de Taylor no tempo $t=100 \mathrm{~s}$. . . . . . . . . . . 115

5.56 Simulação numérica 3D do jato circular oscilante em diferentes tempos. . . . . . 117

5.57 Diagrama esquemático do problema do colapso de uma bloco de fluido com obstáculo. . . . . . . . . . . . . . . . . . . . 118

5.58 Simulação numérica 3D do colapso de uma bloco de fluido com obstáculo em diferentes tempos. . . . . . . . . . . . . . . . . . . . . 119

5.59 Geometria do problema da expansão brusca turbulento. . . . . . . . . . . . . 120

5.60 Perfis da componente $u$ de velocidade do problema da expansão brusca no regime turbulento, mostrando a convergência da solução numérica nas três malhas usando o esquema ADBQUICKEST.

5.61 Problema da expansão brusca ilustrando o perfil da componente $u$ de velocidade com $R e=1.32 \times 10^{5} \ldots \ldots \ldots \ldots \ldots$. . . . . . . . . . . 122

5.62 Problema da expansão brusca ilustrando o perfil da componente $v$ de velocidade com $R e=1.32 \times 10^{5}$.

5.63 Comparação entre as soluções numéricas nas três malhas utilizando o esquema ADBQUICKEST e a solução analítica de Watson. . . . . . . . . . . . . . . . . . 124

5.64 Ampliação das soluções numéricas e da solução analítica de Watson. . . . . . . . 124

5.65 Jato livre 2D no regime turbulento utilizando $R e=5.0 \times 10^{4}$.

5.66 Geometria e parâmetros para o problema de um jato horizontal turbulento penetrando em uma porção de fluido estacionário: $U=2.0 \mathrm{~ms}^{-1}$ e $D=0.05$ m. 
5.67 Evolução dos contornos de pressão de um jato penetrando em uma porção de fluido.

5.68 Evolução dos contornos de velocidade $u$ de um jato penetrando em uma porção de fluido. . . . . . . . . . . . . . . . . . . . . . 128

5.69 Evolução dos contornos de velocidade $v$ de um jato penetrando em uma porção de fluido. . . . . . . . . . . . . . . . . . . . . . 129

5.70 Desenvolvimento do campo de pressão e a evolução do perfil da superfície livre da simulação numérica 2D do problema "sluice gate". . . . . . . . . . . . . . . . 130

5.71 Geometria do problema do colapso de um bloco de fluido. . . . . . . . . . . . 131

5.72 Comparação entre os dados experimentais de Martin e Moyce e Koshizuka e Oka e as soluções numéricas 3D nas quatro malhas da posição frontal do movimento

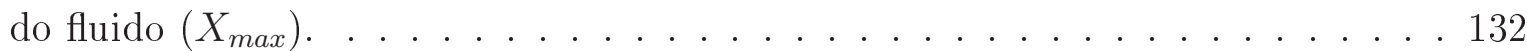

5.73 Comparação entre os os dados experimentais e os resultados numéricos obtidos sem modelagem de turbulência e com o modelo $\kappa-\varepsilon$. . . . . . . . . . . . . . . 133

5.74 Simulação numérica 3D mostrando a evolução da superfície livre em diferentes tempos.

5.75 Simulação numérica 3D mostrando o contorno da pressão em diferentes tempos. 134

5.76 Simulação numérica 3D mostrando o contorno da velocidade na direção $x \mathrm{em}$ diferentes tempos. . . . . . . . . . . . . . . . . . . 135

5.77 Simulação numérica 3D mostrando o contorno da velocidade na direção $z$ em diferentes tempos. . . . . . . . . . . . . . . . . . 136

5.78 Configuração esquemática 2D mostrando a geometria e os parâmetros: $U=2.0$

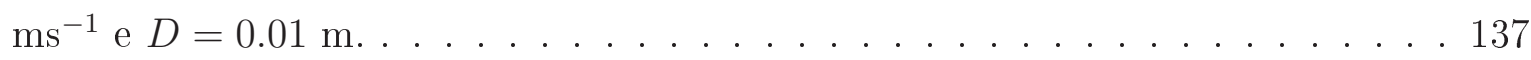

5.79 Soluções numéricas (corte do plano $x z$ ) obtida pelo código Freeflow $3 D$ com modelo $\kappa-\varepsilon$ mostrando: (a) comparação na malha grossa; (b) comparação na malha intermediária; (c) comparação na malha fina; (d) comparação nas três malhas.

5.80 Soluções numéricas (corte do plano yz) obtida pelo código Freeflow 3D com modelo $\kappa-\varepsilon$ mostrando: (a) comparação na malha grossa; (b) comparação na malha intermediária; (c) comparação na malha fina; (d) comparação nas três malhas. . . . . . . . . . . . . . . . . . . . . . . 140

5.81 Simulação numérica 3D de um jato incidindo sobre uma superfície rígida. . . . . 141

5.82 Visualizações do corte do plano $x z$ na posição $y=0.05$ da simulação numérica 3D do jato incidindo sobre uma superfície rígida. . . . . . . . . . . . . . 141

5.83 Visualizações do corte do plano $y z$ na posição $x=0.05$ da simulação numérica 3D do jato incidindo sobre uma superfície rígida no tempo. . . . . . . . . . . . . 142 
5.84 Comparação da simulação numérica obtida pelo código Freeflow $3 D$ equipado com as modelagens ADBQUICKEST e $\kappa-\varepsilon$ e o experimento realizado por Storr e Behnia, para o caso em que $R e=3000$, mostrando: (a) visualização experimental; (b) a visão frontal da simulação numérica; (c) visão 3D da simulação numérica. . . . . . . . . . . . . . . . . . . . 144

5.85 Geometria do problema do colapso de fluido com obstáculo localizado no centro. 146

5.86 Geometria do problema do colapso de fluido com obstáculo localizado junto a parede. . . . . . . . . . . . . . . . . . 146

5.87 Geometria do problema do colapso de fluido com dois obstáculos. . . . . . . . 146

5.88 Simulação numérica 3D usando o esquema ADBQUICKEST e o modelo $\kappa$ $\varepsilon$ do problema interação fluido-estrutura com o obstáculo localizado no centro (Configuração I) . . . . . . . . . . . . . . . . . . . . . . . . . 147

5.89 Simulação numérica 3D usando o esquema ADBQUICKEST e o modelo $\kappa-\varepsilon$ do problema interação fluido-estrutura com o obstáculo localizado junto a parede (Configuração II) . . . . . . . . . . . . . . . . . . . . . . . . . . . . . . . . 148

5.90 Simulação numérica 3D usando o esquema ADBQUICKEST e o modelo $\kappa-\varepsilon$ do problema interação fluido-estrutura com dois obstáculos (Configuração III). 149 


\section{Lista de Tabelas}

5.1 Teste de convergência: erros nas normas $L_{1}, L_{2}$ e $L_{\infty}$ e estimativa da ordem de convergência para os quatros esquemas com $u_{0}=\sin (x),-\pi \leq x \leq \pi$. . . . . 80

5.2 Teste de convergência: erros nas normas $L_{1}, L_{2}$ e $L_{\infty}$ e estimativa da ordem de convergência para os quatros esquemas com $u_{0}(x)=\sin ^{4}(\pi x),-2 \leq x \leq 2$. . . . 81

5.3 Valores do comprimento da recirculação $x_{1}$ dos dados experimentais e numéricos para diferentes números de Reynolds. . . . . . . . . . . . . . . . . . . . . . . 101

5.4 Valores do comprimento da recirculação, $x_{1} \ldots \ldots \ldots$. . . . . . . . . . 121

5.5 Outros valores do comprimento da recirculação. . . . . . . . . . . . . . . . . 121 


\section{Sumário}

1 Introdução $\quad 1$

1.1 Organização da tese . . . . . . . . . . . . . . . . . . . . 3

1.2 Equipamentos utilizados neste trabalho . . . . . . . . . . . . . 5

2 Formulação Matemática $\quad 7$

2.1 Equação linear de advecção $1 \mathrm{D} \ldots \ldots$. . . . . . . . . . . . . . 7

2.2 Equação não linear de Burgers 1D . . . . . . . . . . . . . . . . . . . . 8

2.3 Equações de águas rasas $1 \mathrm{D} \ldots \ldots \ldots$. . . . . . . . . . . . . . . . 8

2.4 Equações de Euler 1D . . . . . . . . . . . . . . . . . . . . . . 8

2.5 Equações de Navier-Stokes . . . . . . . . . . . . . . . . . . . . . . . . 9

2.5.1 Adimensionalização das equações de Navier-Stokes . . . . . . . . . . . . . 10

2.5.2 Modelagem da turbulência . . . . . . . . . . . . . . . . . . 11

2.5.3 Equações de Navier-Stokes com média de Reynolds . . . . . . . . . . . . 12

2.5.4 Condições Iniciais e de Contorno . . . . . . . . . . . . . . . . . . 23

3 Estratégia "Upwind" $\quad 29$

3.1 Aproximações para os termos advectivos . . . . . . . . . . . . . . . . 29

3.2 Restrições TVD . . . . . . . . . . . . . . . . . . . . . . . 31

3.3 Variáveis Normalizadas . . . . . . . . . . . . . . . . . . . . 32

3.4 Critério de limitação CBC . . . . . . . . . . . . . . . . . . . . 33

3.5 O esquema ADBQUICKEST . . . . . . . . . . . . . . . . 35

4 Formulação Numérica $\quad 43$

4.1 A metodologia MAC . . . . . . . . . . . . . . . . . 43

4.1.1 Malha computacional . . . . . . . . . . . . . . . . . 44

4.1 .2 Classificação das células . . . . . . . . . . . . . . . . 45

4.2 Método de projeção . . . . . . . . . . . . . . . . . . 46

4.2.1 Procedimento de solução . . . . . . . . . . . . . . . . . . 46 
4.2.2 Algoritmo computacional . . . . . . . . . . . . . . . . . . 48

4.2 .3 Restrição no valor do passo temporal . . . . . . . . . . . . . . . . . . . 49

4.3 Discretização das Equações . . . . . . . . . . . . . . . . . . . . . . . 50

4.3.1 Discretização dos Termos Advectivos . . . . . . . . . . . . . . . 52

4.3.2 Discretizações das condições de contorno . . . . . . . . . . . . . . 66

5 Resultados Numéricos $\quad 75$

5.1 Simulação de leis de conservação 1D . . . . . . . . . . . . . . . . . . . 75

5.1 .1 Equação linear de advecção . . . . . . . . . . . . . . . . . 75

5.1 .2 Equação de Burgers . . . . . . . . . . . . . . . . . . . . . 82

5.1 .3 Sistemas de equações hiperbólicas . . . . . . . . . . . . . . . . . . . 84

5.1 .4 Comentários . . . . . . . . . . . . . . . . . . . . 97

5.2 Simulação de escoamentos laminares $2 \mathrm{D}$ e 3D $\ldots \ldots \ldots$. . . . . . . . . . 98

$5.2 .1 \quad$ Expansão brusca 2D . . . . . . . . . . . . . . . . . . . . . . . . . 98

5.2.2 Jato livre 2D sobre uma superfície rígida impermeável . . . . . . . . . . . 103

5.2 .3 Colapso de fluido: casos 2D e 3D . . . . . . . . . . . . . 107

5.2.4 Aplicações: escoamentos 3D com superfícies livres móveis . . . . . . . . . 112

5.2 .5 Comentários . . . . . . . . . . . . . . . . . . . . . . 119

5.3 Simulação de escoamentos turbulentos 2D e 3D . . . . . . . . . . . . 120

5.3.1 Expansão brusca 2D . . . . . . . . . . . . . . . . . . . 120

5.3.2 Jato livre 2D sobre uma superfície rígida impermeável . . . . . . . . . . . 123

5.3 .3 Aplicações 2D . . . . . . . . . . . . . . . . . . . . . . . 126

5.3 .4 Colapso de um bloco de fluido . . . . . . . . . . . . . . . . 131

5.3.5 Jato livre sobre uma superfície rígida impermeável . . . . . . . . . . . . 137

5.3 .6 Aplicações 3D . . . . . . . . . . . . . . . . . . . . . . . . 143

5.3 .7 Comentários . . . . . . . . . . . . . . . . . . 150

6 Considerações Finais e Trabalhos Futuros $\quad 151$

$\begin{array}{lr}\text { Referências Bibliográficas } & 167\end{array}$ 
CAPÍTUlo

1

\section{Introdução}

Apesar das equações diferenciais parciais (EDPs) que modelam problemas em dinâmica dos fluidos (Euler e Navier-Stokes) serem bem conhecidas, suas soluções numéricas continuam ainda desafiadoras. Em particular, há dois desafios associados à computação e ao entendimento desses problemas: um deles é a formação de descontinuidades (choques) e o outro é fenômeno turbulência. Ambos os desafios são atribuídos, principalmente, ao tratamento dos termos advectivos não lineares nessas equações de transporte. Dentro deste cenário, propõe-se na presente tese de doutorado apresentar o estudo do desenvolvimento de um novo esquema "upwind" de alta resolução para leis de conservação gerais e sua associação com a modelagem da turbulência a duas equações para a solução de problemas de dinâmica dos fluidos. Dessa forma, o tema do presente trabalho de pesquisa é sobre a solução numérica de leis de conservação gerais e problemas de escoamentos incompressíveis em condições severas de advecção.

Conseguir soluções numéricas representativas para essa classe de problemas é difícil, em virtude da forte influência dos termos não lineares advectivos nas equações de transporte. Consequentemente, a escolha do método numérico que leva em conta a direção do escoamento (vide Li [85] ou Zingg et al. [166] - usualmente conhecido como esquema de advecção "upwind") tem sido uma das principais preocupações da comunidade científica moderna em dinâmica dos fluidos computacional (veja, por exemplo, Hussaini [64]). O problema maior é que, por um lado, ao se usar esquemas "upwind" de primeira ordem para os fluxos numéricos, a solução computada sofre suavização devido à viscosidade numérica adicional (também conhecida por dissipação numérica) e, por outro lado, ao se aplicar esquemas clássicos de alta ordem para esses fluxos, tais como QUICK ("Quadratic Upstream Interpolation for Convective Kinematics") (Leonard [79]), "upwind" de segunda ordem (Warming e Beam [153]), Lax-Wendroff (Lax e Wendroff[77]) ou diferenças centradas, a solução numérica apresenta oscilações não físicas (dispersão numérica), 
principalmente em regiões de altos gradientes, que na maioria das vezes ocasionam instabilidade numérica.

Esquemas computacionais "upwind" limitados de alta resolução (maior ou igual a 2) são técnicas especializadas para aproximar termos advectivos (lineares ou não lineares) de leis de conservação, tais como as equações de Euler da dinâmica dos gases ou as equações de Navier-Stokes para o movimento dos fluidos (ver, por exemplo, White [158]). A idéia básica por trás desses esquemas é usar um esquema numérico tão preciso quanto possível em regiões suaves e, ao mesmo tempo, adicionar dissipação numérica controlada em regiões de gradientes elevados. Seus objetivos principais são manter estabilidade da solução numérica, capturar descontinuidades ou choques (sem a presença de oscilações numéricas ou, em casos extremos, com oscilações limitadas), ser computacionalmente simples e econômico e conseguir convergência.

Para se obter estabilidade com pouca dissipação numérica, várias estratégias "upwind" têm sido desenvolvidas nos últimos anos. O esquema VONOS ("Variable-Order Non-Oscillatory Scheme") (Varonos e Bergeles [149]), por exemplo, introduz pouca viscosidade numérica para o regime laminar confinado, e é capaz de resolver regiões do escoamento onde os gradientes desempenham papel de extrema importância (ver, por exemplo, Castelo et al. [18], Tomé et al. [142], Ferreira et al. [44] e Ferreira et al. [42]). Muitos esquemas "upwind" de alta resolução modernos baseiam-se na restrição TVD ("Total Variation Diminishing") de Harten [55], no critério CBC ("Convection Boundedness Criterion") de Gaskell e Lau [47] e no contexto NVD ("Normalized Variable Diagram") de Leonard [80]. Esquemas representativos desta classe são os esquemas WACEB ("Weighted Average Coefficients Ensuring Boundedness") (Song et al. [126]), CUBISTA ("Convergent and Universally Bounded Interpolation Scheme for the Treatment of Advection") (Alves et al. [2]) e, mais recentemente, o esquema QUICKEST ("Quadratic Upstream Interpolation for Convective Kinematics with Estimated Streaming Terms") adaptativo, também denominado de ADBQUICKEST ("Adaptative Bounded QUICKEST") (Kaibara et al. [66]).

Vê-se, portanto, que a dissipação numérica e a dispersão numérica - ou difusão numérica como um todo (Sheu et al. [119]) - são as principais fontes causadoras de erros na solução computacional de leis de conservação e problemas de escoamentos de fluido. Complicações adicionais aparecem quando se pretende simular numericamente a turbulência nos fluidos com superfícies livres via modelagem da $\kappa-\varepsilon$, pois, além de se exigir uma estratégia de avanço de fronteira, deve-se introduzir duas equações transientes de transporte não lineares (uma para a energia cinética e outra para a taxa de dissipação de energia cinética). Entre os modelos de duas equações encontrados na literatura está o modelo $\kappa-\varepsilon$ padrão (Launder e Spalding [74]), que, quando associado a um bom esquema "upwind" de alta resolução, constitui uma ferramenta poderosa na previsão de escoamentos 3D altamente complexos.

O risco em se obter soluções numéricas de leis de conservação não convergentes, utilizando-se estratégias não limitadas para os termos advectivos, e a necessidade de se resolver escoamentos 
turbulentos 3D com superfícies livres móveis, empregando-se uma modelagem com baixo custo computacional, tem sido a motivação para este trabalho.

Este trabalho tem como objetivo a simulação numérica de leis de conservação 1D e escoamentos incompressíveis com superfícies livres móveis $2 \mathrm{D}$ e 3D, e as principais contribuições são: (i) descrição de um novo esquema "upwind" de alta resolução (o ADBQUICKEST) para resolver problemas transientes envolvendo descontinuidades e a implementação no ambiente de simulação Freeflow $2 D$ e $3 D$ de Castelo et al. [19]; (ii) incorporação da combinação desse novo esquema e a modelagem de turbulência $\kappa-\varepsilon$ padrão de Launder e Spalding [74] no código Freeflow $3 D$ para resolver problemas de escoamentos com superfícies livres; e (iii) apresentação de uma gama bastante significativa de dados de simulações numéricas de leis de conservação 1D e de escoamentos incompressíveis (laminares e turbulentos) com superfícies livres 2D e 3D. Essas contribuições são inovadoras na área de dinâmica dos fluidos computacional, pois visa a análise, a implementação de um novo método numérico e a incorporação de um modelo de turbulência no ambiente de simulação Freeflow 3D.

Alguns dos resultados desta tese já foram publicados na literatura e estão listados a seguir.

- Simulação de Escoamentos de Fluidos Incompressíveis a Altos Reynolds. TEMA -Tendências em Matemática Aplicada e Computacional, v. 7, p. 219-228, 2006;

- A combination of implicit and adaptative upwind tools for the numerical solution of incompressible free surface flows, Communications in Numerical Methods in Engineering, v. 23, p. 419-445, 2007 (DOI: 10.1002/cnm.900);

- Incompressible Turbulent Flow Simulation using the $\kappa-\varepsilon$ Model and Upwind Schemes, Mathematical Problems in Engineering, 2007, Article ID 12741 (DOI:10.1155/2007/12741);

- Evaluation of a bounded high order upwind scheme for 3D incompressible free surface flow computations, Mathematics and Computers in Simulation, v. 79, p. 1895-1914, 2009 (DOI:10.1016/j.matcom.2007.04.018);

- Assessment of a high-order finite difference upwind scheme for the simulation of convectiondiffusion problems, International Journal for Numerical Methods in Fluids, 2008, aceito para publicação (DOI: 10.1002/fld.1875).

\subsection{Organização da tese}

A presente tese está organizada da seguinte maneira:

\section{- Capítulo 2: Formulação Matemática}

Nesse capítulo são apresentadas as EDPs básicas, em particular, a equação linear de adveç̧ão 1D, a equação não linrear de Burgers 1D, sistemas hiperbólicos 1D (águas rasas e 
Euler), e as equações de Navier-Stokes na formulação incompressível. São apresentadas também o processo de adimensionalização das equações de Navier-Stokes e uma breve introdução sobre a modelagem da turbulência a duas equações. Além disso, são apresentadas as condições iniciais e de contorno para os problemas de escoamento de fluidos.

\section{- Capítulo 3: Estratégia "Upwind"}

Neste capítulo, é apresentado a estratégia "upwind" ADBQUICKEST adotada neste trabalho. Discutem-se as propriedades físicas e os aspectos teóricos fundamentais dos termos advectivos utilizando-se os conceitos de variáveis normalizadas (NV) de Leonard e o critério de limitação (CBC) de Gaskell e Lau. Discute-se, também, as restrições TVD de Harten.

\section{- Capítulo 4: Formulação Numérica}

Neste capítulo, é apresentada uma breve descrição do método MAC para a solução numérica de escoamentos incompressíveis transientes com superfícies livres móveis. São discutidos, também, o método projeção de Chorin, o procedimento computacional de solução das equações discretas e a discretização das equações de conservação. Para finalizar, são apresentadas as discretizações dos termos advectivos, das condições de contorno na superfície livre e das variáveis turbulentas adjacentes aos contornos rígidos.

\section{- Capítulo 5: Resultados Numéricos}

Nesta capítulo são apresentados resultados de simulações numéricas das leis de conservação 1D utilizando-se o esquema ADBQUICKEST. Em particular são considerados os seguintes problemas transientes: advecção de escalares, problemas de Riemann para as equações de Burgers, de Euler e águas rasas. Os resultados numéricos de simulações de escoamentos laminares 2D e 3D utilizando-se o esquema ADBQUICKEST implementado nos ambientes de simulações Freeflow 2D e 3D também são apresentados neste capítulo. Em particular, seu desempenho é examinado na solução dos seguintes problemas: expansão brusca 2D, jato livre 2D incidindo sobre uma superfície rígida e colapso de fluido em repouso (casos 2D e 3D). Além desses problemas, algumas aplicações também são apresentadas, como por exemplo, o ressalto hidráulico circular, experimento de Taylor, jato oscilante e o colapso de um bloco de fluido com obstáculo. Também são apresentados os resultados obtidos com os códigos Freeflow $2 D$ e $3 D$ atualizados com o esquema ADBQUICKEST e o modelo de turbulência $\kappa-\varepsilon$ das simulações de problemas de escoamentos incompressíveis 2D e 3D em regime turbulento envolvendo superfícies livres. Em particular, são considerados expansão brusca 2D, jato livre 2D e 3D e colapso de um bloco de fluido. Para finalizar são consideradas algumas aplicações como experimento de Storr e Behnia e o colapso de um bloco de fluido com obstáculos. 
- Capítulo 6: Considerações Finais e Trabalhos Futuros

Neste último capítulo são apresentadas as considerações finais e algumas sugestões para trabalhos futuros.

\subsection{Equipamentos utilizados neste trabalho}

Todas as simulações numéricas apresentadas neste trabalho foram realizadas nas seguintes máquinas do Instituto de Ciências Matemáticas e de Computação (ICMC) da Universidade de São Paulo (USP):

- Um microcomputador Core 2 Duo E8400 3.0 GHz, 4 Gb de memória RAM, 320 Gb de disco rígido e sistema operacional linux 2.6.24.

- Uma estação de trabalho com dois processadores Quad-Core Intel(R) Xeon(R) E5345 2.33GHz, $32 \mathrm{~Gb}$ de RAM, 2 HD de 1Tb em RAID0 e sistema operacional linux 2.6.24.

- Um cluster constituído de: 16 nós dual core AMD Opteron 242-1.6 GHz e 2Gb de RAM, 2 nós Quad Core AMD Opteron 844-1.8 GHz e 8Gb de RAM e 16 nós com dois processadores Quad-Core Intel(R) Xeon(R) E5345 2.33GHz e 16 Gb de RAM. 


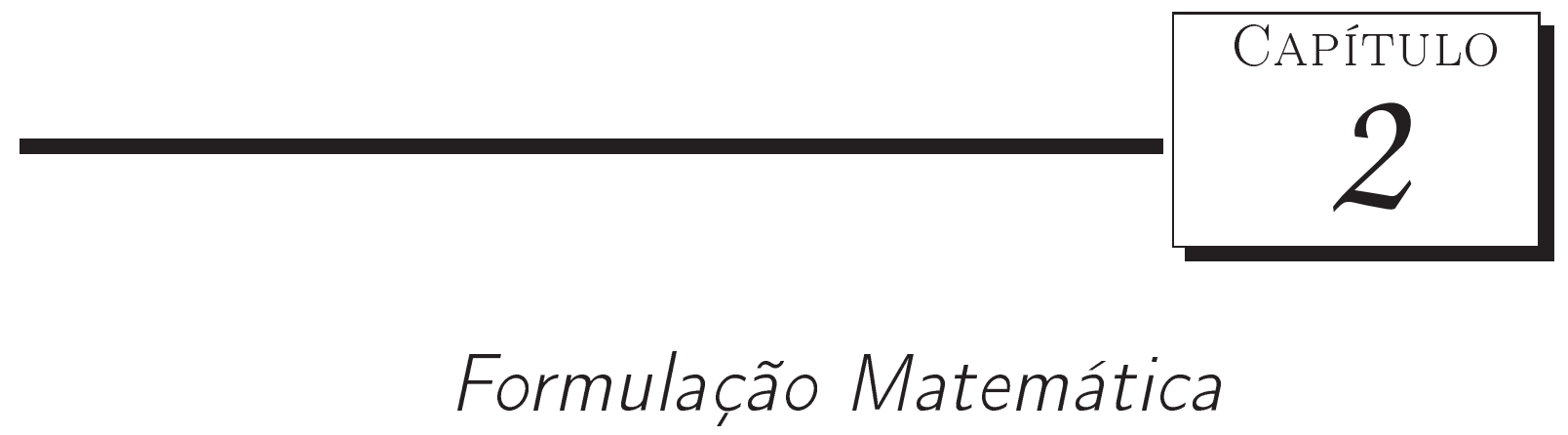

Nesse capítulo são apresentadas as EDPs básicas consideradas neste trabalho, em particular, a equação linear de advecção 1D, a equação não linear de Burgers 1D, sistemas hiperbólicos 1D (águas rasas e Euler), e as equações de Navier-Stokes na formulação incompressível. São apresentadas também o processo de adimensionalização das equações de Navier-Stokes e uma breve introdução sobre a modelagem da turbulência a duas equações. Além disso, são apresentadas as condições iniciais e de contorno para os problemas de escoamento de fluidos.

\subsection{Equação linear de advecção 1D}

Um dos problemas mais simples decorrentes das leis de conservação é equação linear de advecção. Para a simulação do transporte de escalares, o seguinte modelo é adotado

$$
\frac{\partial u}{\partial t}+a \frac{\partial u}{\partial x}=0, \quad a=\text { constante }>0
$$

com as condições iniciais e de contorno dadas, respectivamente, por

$$
\begin{gathered}
u(x, 0)=u_{0}(x), \quad x \in\left[x_{L}, x_{R}\right], \\
u\left(x_{L}, t\right)=0, \quad u\left(x_{R}, t\right)=0,
\end{gathered}
$$

em que $t$ é o tempo e $u=u(x, t)$ é a variável transportada. A solução exata da equação (2.1) é dada por $u(x, t)=u_{0}(x-a t)$. 


\subsection{Equação não linear de Burgers 1D}

A equação não linear de Burgers 1D com viscosidade constante $\nu>0$ é dada por

$$
\frac{\partial u}{\partial t}+\frac{\partial}{\partial x}\left(\frac{u^{2}}{2}\right)=\nu \frac{\partial^{2} u}{\partial x^{2}}, \quad t>0
$$

em que $u=u(x, t)$ é a variável dependente, semelhante à velocidade num escoamento incompressível. As condições iniciais e de contorno são dadas, respectivamente, por

$$
\begin{gathered}
u(x, 0)=u_{0}(x), \quad x \in\left[x_{L}, x_{R}\right], \\
u\left(x_{L}, t\right)=f\left(x_{L}, t\right), \quad u\left(x_{R}, t\right)=g\left(x_{R}, t\right) .
\end{gathered}
$$

É bem conhecido na literatura que, para $\nu>0$, o termo da viscosidade produz soluções suaves e a energia do sistema dissipa suavemente. Entretanto, para $\nu \rightarrow 0$, descontinuidades (choques) podem se desenvolver na solução, até mesmo, para condições iniciais suaves.

A equação de Burgers é um modelo interessante que inclui uma combinação de efeitos de advecção (não lineares) e efeitos viscosos (lineares), sendo, portanto, essencial no estudo da dinâmica dos fluidos. Ela serve também como um modelo simplificado para o entendimento da formação do choque e da turbulência nos fluidos (ver, por exemplo, Karpman [67]).

\subsection{Equações de águas rasas 1D}

O sistema hiperbólico de equações de águas rasas (LeVeque [82]) é dado por

$$
\frac{\partial}{\partial t}\left(\begin{array}{c}
h \\
h u
\end{array}\right)+\frac{\partial}{\partial x}\left(\begin{array}{c}
h u \\
h u^{2}+\frac{1}{2} g h^{2}
\end{array}\right)=0
$$

em que $h=h(x, t)$ é a profundidade do fluido no canal, $u=u(x, t)$ é a velocidade na direção horizontal e $g$ é a gravidade. As condições iniciais são dadas por

$$
u(x, 0)=u_{0}(x) \quad \text { e } \quad h(x, 0)=h_{0}(x), \quad x \in\left[x_{L}, x_{R}\right]
$$

e com condições homogêneas de Neumann no contorno.

\subsection{Equações de Euler 1D}

A lei de conservação hiperbólica que modela, por exemplo, o problema do tubo de choque é o sistema de equações não lineares de Euler 1D da dinâmica dos gases dada por

$$
\frac{\partial \mathbf{u}}{\partial t}+\frac{\partial \mathbf{F}(\mathbf{u})}{\partial x}=0
$$


Nessas equações, $\mathbf{u}=(\rho, \rho u, E)^{T}$ é o vetor das variáveis conservadas e $\mathbf{F}(\mathbf{u})=\left(\rho u, \rho u^{2}+\right.$ $p, u(E+p))^{T}$ é o vetor de fluxo. As variáveis $\rho, u, \rho u, E, p$ são, respectivamente, a densidade, a velocidade, a quantidade de movimento, a energia total e a pressão. Além disso, para fechar o sistema, é considerada a equação do gás ideal $p=(\gamma-1)\left(E-\frac{1}{2} \rho u^{2}\right)$, na qual $\gamma=1.4$ é a razão do calor específico a pressão constante pelo calor específico a volume constante (Harten [56]). As condições inicias para essas equações são dadas por

$$
\mathbf{u}(x, 0)=\mathbf{u}_{0}(x)=\left\{\begin{array}{l}
\left(\rho_{L}^{0}, u_{L}^{0}, p_{L}^{0}\right)^{T}, \quad x \leq x_{0}, \\
\left(\rho_{R}^{0}, u_{R}^{0}, p_{R}^{0}\right)^{T}, x>x_{0},
\end{array} \quad x \in\left[x_{L}, x_{R}\right],\right.
$$

e as condições de contorno por (ver detalhes em Toro [145])

$$
\mathbf{u}\left(x_{L}, t\right)=\mathbf{u}_{L}(t) \quad \text { e } \quad \mathbf{u}\left(x_{R}, t\right)=\mathbf{u}_{R}(t)
$$

\subsection{Equações de Navier-Stokes}

O movimento de fluidos newtonianos incompressíveis é regido pelas equações de Navier-Stokes incompressíveis, que, na forma conservativa podem ser escritas como

$$
\begin{gathered}
\nabla \cdot \mathbf{u}=0 \\
\frac{\partial \mathbf{u}}{\partial t}+\nabla \cdot(\mathbf{u u})=-\frac{1}{\rho} \nabla p+\nu \nabla^{2} \mathbf{u}+\mathbf{g},
\end{gathered}
$$

em que $\mathbf{u}=\mathbf{u}(\mathbf{x}, t)$ denota o vetor velocidade de uma partícula idealizada de fluido localizada na posição $\mathbf{x}$ e no instante de tempo $t, p=p(\mathbf{x}, t)$ é a pressão, g é o campo gravitacional e $\nu$ é o coeficiente de viscosidade cinemática do fluido dado por $\nu=\mu / \rho$ ( $\rho$ é a densidade e $\mu$ é a viscosidade do fluido).

Outra forma para escrever as equações de conservação (2.5) e (2.6) é utilizando a notação de Einstein, que no caso 3D tornam-se

$$
\begin{gathered}
\frac{\partial u_{i}}{\partial x_{i}}=0 \\
\frac{\partial u_{i}}{\partial t}+\frac{\partial\left(u_{i} u_{j}\right)}{\partial x_{j}}=-\frac{1}{\rho} \frac{\partial p}{\partial x_{i}}+\nu \frac{\partial}{\partial x_{j}}\left(\frac{\partial u_{i}}{\partial x_{j}}\right)+g_{i}, \quad i=1,2,3 .
\end{gathered}
$$

Em coordenadas cartesianas 3D, as equações (2.5) e (2.6) podem ser escritas como

$$
\frac{\partial u}{\partial x}+\frac{\partial v}{\partial y}+\frac{\partial w}{\partial z}=0
$$




$$
\begin{gathered}
\frac{\partial u}{\partial t}+\frac{\partial(u u)}{\partial x}+\frac{\partial(u v)}{\partial y}+\frac{\partial(u w)}{\partial z}=-\frac{1}{\rho} \frac{\partial p}{\partial x}+\nu\left(\frac{\partial^{2} u}{\partial x^{2}}+\frac{\partial^{2} u}{\partial y^{2}}+\frac{\partial^{2} u}{\partial z^{2}}\right)+g_{x}, \\
\frac{\partial v}{\partial t}+\frac{\partial(v u)}{\partial x}+\frac{\partial(v v)}{\partial y}+\frac{\partial(v w)}{\partial z}=-\frac{1}{\rho} \frac{\partial p}{\partial y}+\nu\left(\frac{\partial^{2} v}{\partial x^{2}}+\frac{\partial^{2} v}{\partial y^{2}}+\frac{\partial^{2} v}{\partial z^{2}}\right)+g_{y}, \\
\frac{\partial w}{\partial t}+\frac{\partial(w u)}{\partial x}+\frac{\partial(w v)}{\partial y}+\frac{\partial(w w)}{\partial z}=-\frac{1}{\rho} \frac{\partial p}{\partial z}+\nu\left(\frac{\partial^{2} w}{\partial x^{2}}+\frac{\partial^{2} w}{\partial y^{2}}+\frac{\partial^{2} w}{\partial z^{2}}\right)+g_{z},
\end{gathered}
$$

em que $g_{x}, g_{y}$ e $g_{z}$ são as componentes do campo gravitacional nas direções $x, y$ e $z$ respectivamente.

\subsubsection{Adimensionalização das equações de Navier-Stokes}

Em geral, considera-se uma mudança de variáveis, surgindo alguns grupos adimensionais como por exemplo, os números de Reynolds e Froude.

O número de Reynolds representa a razão entre as forças inerciais e as forças viscosas do escoamento na qual é definido por

$$
R e=\frac{\rho L U}{\mu}=\frac{L U}{\nu}
$$

em que $L$ é uma escala de comprimento e $U$ uma escala de velocidade.

O número de Froude representa a razão entre as forças inerciais e as forças gravitacionais e é definido por

$$
F r=\frac{U}{\sqrt{g L}}
$$

Esses dois números adimensionais são os parâmetros fundamentais em escoamentos incompressíveis envolvendo superfícies livres.

Neste trabalho, para adimensionalizar as equações de quantidade de movimento e continuidade define-se as variáveis adimensionais como

$$
\overline{\mathbf{u}}=\frac{\mathbf{u}}{U}, \quad \overline{\mathbf{x}}=\frac{\mathbf{x}}{L}, \quad \bar{t}=\frac{U t}{L}, \quad \bar{p}=\frac{p}{\rho U^{2}}, \quad \overline{\mathbf{g}}=\frac{\mathbf{g}}{g} .
$$

Utilizando-se as transformações (2.13) e eliminando-se as "barras" para simplificar, as equações de conservação de massa e conservação de movimento na forma adimensional e conservativa são dadas por

$$
\begin{aligned}
\nabla \cdot \mathbf{u} & =0 \\
\frac{\partial \mathbf{u}}{\partial t}+\nabla \cdot(\mathbf{u u}) & =-\nabla p+\frac{1}{R e} \nabla^{2} \mathbf{u}+\frac{1}{F r^{2}} \mathbf{g} .
\end{aligned}
$$

As equações (2.14) e (2.15) podem ser formuladas na forma adimensional e cartesiana 3D 
como

$$
\begin{gathered}
\frac{\partial u}{\partial x}+\frac{\partial v}{\partial y}+\frac{\partial w}{\partial z}=0 \\
\frac{\partial u}{\partial t}+\frac{\partial(u u)}{\partial x}+\frac{\partial(u v)}{\partial y}+\frac{\partial(u w)}{\partial z}=-\frac{\partial p}{\partial x}+\frac{1}{R e}\left(\frac{\partial^{2} u}{\partial x^{2}}+\frac{\partial^{2} u}{\partial y^{2}}+\frac{\partial^{2} u}{\partial z^{2}}\right)+\frac{1}{F r^{2}} g_{x} \\
\frac{\partial v}{\partial t}+\frac{\partial(v u)}{\partial x}+\frac{\partial(v v)}{\partial y}+\frac{\partial(v w)}{\partial z}=-\frac{\partial p}{\partial y}+\frac{1}{R e}\left(\frac{\partial^{2} v}{\partial x^{2}}+\frac{\partial^{2} v}{\partial y^{2}}+\frac{\partial^{2} v}{\partial z^{2}}\right)+\frac{1}{F r^{2}} g_{y} \\
\frac{\partial w}{\partial t}+\frac{\partial(w u)}{\partial x}+\frac{\partial(w v)}{\partial y}+\frac{\partial(w w)}{\partial z}=-\frac{\partial p}{\partial z}+\frac{1}{R e}\left(\frac{\partial^{2} w}{\partial x^{2}}+\frac{\partial^{2} w}{\partial y^{2}}+\frac{\partial^{2} w}{\partial z^{2}}\right)+\frac{1}{F r^{2}} g_{z}
\end{gathered}
$$

As equações (2.16), (2.17), (2.18) e (2.19) constituem um sistema de equações, sem modelagem de turbulência, que serão usadas para simular escoamentos em regime laminar.

\subsubsection{Modelagem da turbulência}

Vários escoamentos encontrados na natureza e indústria, tais como jatos, esteiras, escoamentos em dutos, camada-limite sobre superfícies sólidas e escoamentos 3D complexos, tornam-se instáveis para o número de Reynolds maior que um valor crítico determinado (ver Ames [3]). Abaixo desse valor, os escoamentos são bem comportados, e acima do valor crítico eles tornam-se desordenados. Nesse último caso, se os escoamentos realizam eficiente mistura e transporte de propriedades físicas e tem vorticidade distribuída irregularmente em todas as direções, eles são "definidos" como escoamentos turbulentos. Os escoamentos tipicamente turbulentos são transientes, 3D e contêm flutuações na velocidade aparentemente aleatórias com uma ampla faixa de escalas de comprimento e de tempo. O intervalo de escalas no regime é muito extenso contendo desde as estruturas turbilhonares menores, com dimensões da ordem das microescalas dissipativas de Kolmogorov (Tennekes e Lumley [137]), até as estruturas turbilhonares maiores (coerentes), caracterizadas pela geometria do problema.

Na literatura destacam-se três principais abordagens para simulações de escoamentos incompressíveis no regime turbulento (Mellor e Herring [96]): a DNS ("Direct Numerical Simulation"); a LES ("Large-Eddy Simulation") e a RANS ("Reynolds-Averaged Navier-Stokes"). Na metodologia DNS, todas as escalas da turbulência são computadas numericamente, a partir das equações de Navier-Stokes e continuidade, sem levar em consideração o fato de que as pequenas e as grandes escalas de turbulência possuem características físicas diferentes. A metodologia LES é formulada com base num processo de filtragem das equações de conservação em que as escalas maiores ("grid-scale") de turbulência são calculadas diretamente e os efeitos das estruturas de pequenas escalas ("subgrid-scale") são modelados. A abordagem RANS, em que a média de Reynolds é um caso particular de filtro, é utilizada para simplificar a modelagem "submalha", ou seja, informações sobre flutuações turbulentas são obtidas do tensor de Reynolds.

Neste trabalho, para a simulação de escoamentos incompressíveis turbulentos será empre- 
gada a metodologia RANS. Em particular, adota-se a família de modelos baseados no conceito de viscosidade turbulenta por Boussinesq [13].

\subsubsection{Equações de Navier-Stokes com média de Reynolds}

Nos escoamentos incompressíveis em regime turbulento, as variáveis de campo $\mathbf{u}$ e $p$ são funções aleatórias do tempo e espaço, e até o presente momento não se conhecem tais funções que satisfaçam às equações (2.16)-(2.19).

A aleatoriedade da dinâmica turbulenta, juntamente com seu número praticamente infinito de graus de liberdade, levou a idéia de que somente tratamentos estatísticos seriam capazes de formular modelos preditivos de escoamentos. Esta visão começou a se estabelecer na comunidade científica primordialmente a partir de um artigo de Osborne Reynolds [111], publicado ainda no século XIX. Neste trabalho, Reynolds concluiu que fenômenos turbulentos eram demasiadamente complexos para que houvesse qualquer esperança de um entendimento detalhado de sua dinâmica. Por esse motivo, Reynolds reformulou o modelo em termos de variáveis médias em um intervalo de tempo, introduzindo uma decomposição dos parâmetros, denominada de decomposição de escalas de Reynolds, que caracterizam o escoamento (velocidade, pressão, temperatura, etc) em componentes "médias" e "flutuantes" (estocásticas)

$$
\phi(\mathbf{x}, t)=\bar{\phi}(\mathbf{x}, t)+\phi^{\prime}(\mathbf{x}, t)
$$

em que $\bar{\phi}(\mathbf{x}, t)$ é a componente média (estatisticamente estacionária) e $\phi^{\prime}(\mathbf{x}, t)$ a componente flutuante. A razão para tal decomposição é abrir a possibilidade de tratar as propriedades dos escoamentos turbulentos em termos de soluções bem comportadas $\bar{\phi}(\mathbf{x}, t)$, e de contribuições flutuantes de caráter complexo, as quais são tratadas separadamente.
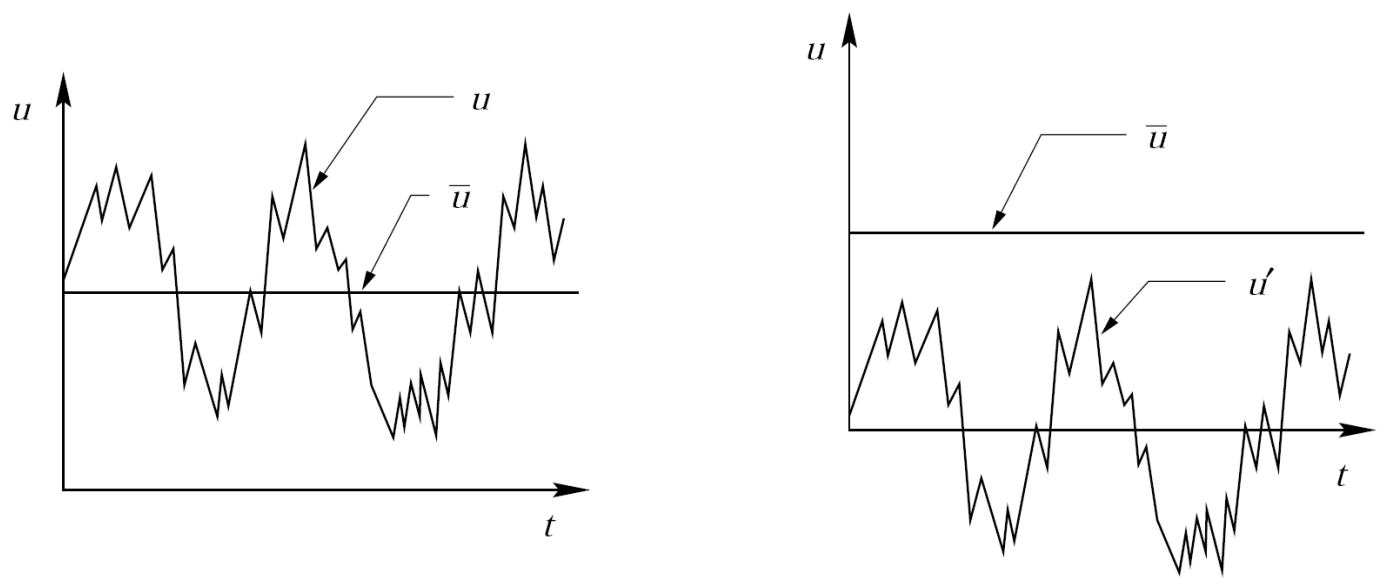

Figura 2.1: A decomposição de Reynolds para o campo de velocidade $u(\mathbf{x}, t)$.

Na decomposição de escalas de Reynolds, pode-se observar que, é a componente flutuante que essencialmente carrega toda a dinâmica do escoamento via sua dependência temporal, 
ou seja, esta componente é a responsável pela modelagem das flutuações características dos escoamentos turbulentos.

Reynolds propôs a existência de uma escala de tempo característica $T$ sobre a qual a média temporal deveria ser tomada. Dessa forma, a decomposição de Reynolds (2.20) é usualmente baseada em médias temporais, sendo definidas por

$$
\bar{\phi}(\mathbf{x}, t)=\frac{1}{T} \int_{t}^{t+T} \phi(\mathbf{x}, \tau) d \tau, \quad T_{0}<<T<<T_{1},
$$

em que o intervalo de tempo $T$ é suficientemente grande com respeito à escala de tempo $T_{0}$ das flutuações turbulentas, porém pequeno em relação à escala de tempo $T_{1}$ dos outros efeitos com respeito ao valor médio. Como consequência desta definição, tomando-se a média temporal da decomposição (2.20), segue que a média das flutuações $\phi^{\prime}(\mathbf{x}, t)$ é zero, ou seja, $\overline{\phi^{\prime}(\mathbf{x}, t)}=0$.

A decomposição de escalas de Reynolds representa uma maneira de introduzir uma formulação estatística para a descrição da dinâmica dos escoamentos turbulentos, possibilitando, assim, sua modelagem numérica. Os detalhes sobre essa decomposição estão bem explicadas em Wilcox [159] e Dubois et al. [31].

As equações de Navier-Stokes escritas em termos da decomposição de escalas de Reynolds, para o campo das velocidades instantâneas $\left(u=\bar{u}+u^{\prime}\right)$, são dadas por

$$
\begin{gathered}
\frac{\partial \bar{u}_{i}}{\partial x_{i}}=0 \\
\frac{\partial \bar{u}_{i}}{\partial t}+\frac{\partial\left(\bar{u}_{i} \bar{u}_{j}\right)}{\partial x_{j}}=-\frac{1}{\rho} \frac{\partial \bar{p}}{\partial x_{i}}+\frac{\partial}{\partial x_{j}}\left(\nu \frac{\partial \bar{u}_{i}}{\partial x_{j}}-\overline{u_{i}^{\prime} u_{j}^{\prime}}\right)+g_{i}, \quad i=1,2,3,
\end{gathered}
$$

em que $\overline{u_{i}^{\prime} u_{j}^{\prime}}$ representa os efeitos da turbulência sobre o escoamento médio $\bar{u}_{i}$ e é chamado de tensões de Reynolds ou tensões turbulentas, que resulta das não-linearidades presentes nas equações do movimento. As equações (2.21) e (2.22) são usualmente chamadas de equações médias de Reynolds.

As tensões de Reynolds são cruciais para a modelagem dos escoamentos turbulentos, afinal elas representam as flutuações estocásticas nas equações de Reynolds. O tensor de tensões de Reynolds consiste em um tensor de segunda ordem simétrico, isto é, $\overline{u_{i}^{\prime} u_{j}^{\prime}}=\overline{u_{j}^{\prime} u_{i}^{\prime}}$. Logo, a obtenção da média temporal das equações médias resulta na introdução de seis incógnitas adicionais: $\overline{u^{\prime} u^{\prime}}, \overline{v^{\prime} v^{\prime}}, \overline{w^{\prime} w^{\prime}}, \overline{u^{\prime} v^{\prime}}, \overline{u^{\prime} w^{\prime}}$ e $\overline{v^{\prime} w^{\prime}}$, o que configura um problema matematicamente indeterminado, ou de fechamento, ao se dispor de um número de equações menor do que o número de incógnitas. Consequentemente, as tensões de Reynolds precisam ser modeladas por equações adicionais de modo a se obter o fechamento do problema. Embora existam na literatura uma variedade de modelos prescrevendo relações não-lineares (Speziale [128]) para o fechamento das equações governantes do escoamento turbulento, o modelo mais popular utiliza a aproximação de Boussinesq, que relaciona, empiricamente, o tensor de Reynolds com o campo 
médio de velocidade.

\subsubsection{Aproximação de Boussinesq}

O primeiro modelo para a descrição matemática dos termos de tensões de Reynolds foi apresentado por Boussinesq [13]. Boussinesq propôs que o tensor de tensões de Reynolds é proporcional ao tensor médio de deformações mais a contribuição média da energia cinética devida ao movimento turbulento, em que a constante de proporcionalidade é conhecida como viscosidade turbulenta ("eddy-viscosity"). Além disso, tal relação constitutiva deve ser consistente com leis físicas bem estabelecidas.

Comparando-se as equações médias e instantâneas do movimento (Ferreira [38]), o tensor de tensões de Reynolds pode ser interpretado como tensões sobre as partículas materiais em adição às tensões determinadas pela pressão e tensões viscosas. Logo, o tensor médio de tensões em um escoamento turbulento pode ser aproximado por

$$
\bar{\sigma}_{i j} \approx-\bar{p} \delta_{i j}+\nu \bar{D}_{i j}-\overline{u_{i}^{\prime} u_{j}^{\prime}}
$$

em que $\bar{D}_{i j}$ é o tensor médio de deformações definido por

$$
\bar{D}_{i j}=\frac{\partial \bar{u}_{i}}{\partial x_{j}}+\frac{\partial \bar{u}_{j}}{\partial x_{i}}
$$

Num escoamento incompressível e em regime turbulento, Boussinesq assumiu que o tensor médio de tensões pode ser representado como

$$
\bar{\sigma}_{i j}=\left(-\bar{p} \delta_{i j}+\nu \bar{D}_{i j}\right)+\left(-\bar{p}_{t} \delta_{i j}+\nu_{t} \bar{D}_{i j}\right)
$$

na qual $\bar{p}_{t}$ é a pressão turbulenta e $\nu_{t}$ a viscosidade turbulenta.

Ao contrário da viscosidade molecular, $\nu$, a viscosidade turbulenta, $\nu_{t}$, não é uma propriedade do fluido, mas do escoamento, devendo portanto, embutir em sua formulação parâmetros que caracterizem adequadamente as tensões turbulentas. Além disso, a viscosidade turbulenta não se origina da viscosidade molecular, mas sim das estruturas turbilhonares produzidas pela turbulência, na qual depende das características do campo de escoamento.

Ao longo dos últimos anos, a hipótese de Boussinesq, em uma forma generalizada proposta por Kolmogorov em 1942, tem sido amplamente utilizada para modelagem de escoamentos turbulentos. Segundo Kolmogorov, o tensor de Reynolds é expresso por

$$
-\overline{u_{i}^{\prime} u_{j}^{\prime}}=\nu_{t} \bar{D}_{i j}-\frac{2}{3} \kappa \delta_{i j}=\nu_{t}\left(\frac{\partial \bar{u}_{i}}{\partial x_{j}}+\frac{\partial \bar{u}_{j}}{\partial x_{i}}\right)-\frac{2}{3} \kappa \delta_{i j},
$$

em que $\delta_{i j}$ representa o delta de Kronecker e $\kappa$ uma grandeza instantânea, relacionada às flutuações turbulentas e que mede a intensidade das flutuações de velocidade. A função escalar $\kappa$ 
foi introduzida por Boussinesq, em analogia com a pressão produzida por flutuações moleculares, dada por

$$
\kappa=\frac{1}{2} \overline{u_{i}^{\prime} u_{i}^{\prime}}
$$

A relação (2.26) também é conhecida na literatura como aproximação de Boussinesq.

Os modelos de turbulência baseados na hipótese de Boussinesq requerem, essencialmente, a avaliação do valor da viscosidade turbulenta, $\nu_{t}$. Em termos dimensionais, a viscosidade turbulenta pode ser calculada como um produto entre a escala de velocidade, $u_{t}$, e a escala de comprimento, $l_{t}$, ou seja,

$$
\nu_{t} \approx u_{t} l_{t}
$$

A análise do espectro de energia cinética turbulenta demonstra que a maior parcela de energia está contida nas grandes escalas. Dessa forma, o comprimento da turbulência, $l_{t}$, dever ser capaz de representar adequadamente a relação entre as estruturas presentes nas grandes escalas e o campo médio do escoamento. O problema, naturalmente, é encontrar meios adequados para a medição de $u_{t}$ e $l_{t}$ (Tannehill et al. [133]).

A viscosidade turbulenta na forma adimensional é dada por

$$
\overline{\nu_{t}}=\frac{\nu_{t}}{\nu}
$$

Substituindo-se a aproximação de Boussinesq (2.26) em (2.22) e utilizando-se as transformações (2.13) e (2.28) obtêm-se as equações adimensionais de Navier-Stokes com média de Reynolds, ou, simplesmente, equações médias de Navier-Stokes,

$$
\begin{gathered}
\frac{\partial u_{i}}{\partial x_{i}}=0 \\
\frac{\partial u_{i}}{\partial t}+\frac{\partial\left(u_{i} u_{j}\right)}{\partial x_{j}}=-\frac{\partial p_{e}}{\partial x_{i}}+\frac{1}{R e} \frac{\partial}{\partial x_{j}}\left(\frac{\partial u_{i}}{\partial x_{j}}\right)+\frac{1}{F r^{2}} g_{i}+\frac{1}{R e} \frac{\partial}{\partial x_{j}}\left(\nu_{t} D_{i j}\right), \quad i=1,2,3,
\end{gathered}
$$

em que $p_{e}$ é a pressão efetiva média, calculada por

$$
p_{e}=p+p_{t}=p+\frac{2}{3} \frac{1}{R e} \kappa .
$$

Nas equações (2.29) e (2.30), o símbolo para média ("a barra") foi eliminado por simplicidade. Essas equações constituem um sistema de EDPs que contêm três equações e quatro incógnitas e, portanto, necessitam de mais uma hipótese adicional para o fechamento. Assim, no contexto da hipótese de Boussinesq, o problema de fechamento da dinâmica média é encontrar uma equação para $\nu_{t}$ em função da posição e do tempo.

Nos modelos de turbulência em que se assume a existência de uma viscosidade adicional, $\nu_{t}$ é prescrita algebricamente em função de outras grandezas derivadas de equações diferenciais. Dependendo do número e do tipo das equações empregadas, os modelos de fechamento podem 
ser normalmente, classificados como (Wilcox [159]):

- Modelos algébricos: Esses modelos são baseados na hipótese de Boussinesq, que estabelece o conceito de viscosidade turbulenta, $\nu_{t}$. Nesses modelos, uma equação algébrica baseada em escalas turbulentas características, é empregada para a determinação do valor da viscosidade turbulenta.

- Modelos a uma equação: Nesses modelos, uma equação diferencial de transporte é resolvida para uma determinada propriedade turbulenta. Em geral, a energia cinética turbulenta $\kappa$ é adotada como a propriedade turbulenta de referência. Uma segunda propriedade, normalmente uma escala de comprimento, é então fornecida por meio de uma expressão algébrica. Os modelos a uma equação empregam, igualmente, a hipótese de viscosidade turbulenta.

- Modelos a duas equações: Os modelos a duas equações utilizam duas equações diferenciais de transporte de propriedades turbulentas, configurando-se, como modelos de fechamento completos. Esses modelos adotam, tipicamente, uma equação de transporte para a energia cinética turbulenta, $\kappa$, juntamente com uma equação de transporte para a taxa de dissipação de energia cinética turbulenta por unidade de massa, $\varepsilon$, ou mesmo, em alguns casos, frequência de passagem de grandes estruturas turbulentas, $\omega$. Tais modelos, em conjunto com os modelos algébricos e os modelos a uma equação, formam os chamados modelos de viscosidade turbulenta.

- Modelos para as tensões de Reynolds: Também chamados de modelos de fechamento de segunda ordem, esses modelos utilizam equações de transporte explícitas para os componentes do tensor de Reynolds. Entretanto, mesmos nesses modelos, ainda se faz necessária a adoção de uma equação de transporte complementar para $\varepsilon$ ou $\omega$ de modo a se obter o fechamento completo do problema.

No presente trabalho foram adotados os modelos a duas equações, em particular, o modelo $\kappa-\varepsilon$, pois segundo a literatura especializada, eles simulam escoamentos complexos muito melhor que os modelos algébricos e a uma equação. Além disso, os modelos a duas equações são significativamente mais simples do que os modelos de alta ordem. Entretanto, a grande dificuldade na sua implementação é o tratamento das regiões próximas aos contornos rígidos, onde a viscosidade molecular exerce influência decisiva na estrutura do movimento.

Uma grande variedade de modelos de turbulência do tipo $\kappa-\varepsilon$ estão disponíveis na literatura e os mais populares em aplicações práticas são os modelos de Launder e Spalding [74] e os modelos de Yakhot e Orszag [160] e Yakhot et al. [161].

\subsubsection{Modelo $\kappa-\varepsilon$ de turbulência}

O modelo $\kappa-\varepsilon$ representa o modelo a duas equações amplamente difundido, sendo considerado o modelo de turbulência padrão em simulações industriais. Este modelo tem sido 
extensivamente validado para diversas aplicações de interesse prático, tendo mostrado um bom equilíbrio entre precisão e robustez. A idéia central para a derivação do modelo $\kappa-\varepsilon$ é acoplar junto às equações médias de Navier-Stokes duas equações diferenciais de transporte, para descrever a evolução da viscosidade adicional. Uma dessas equações governa a distribuição da energia cinética turbulenta, $\kappa$; a outra governa uma característica turbulenta de dimensão diferente

$$
\varepsilon=\nu\left(\overline{\frac{\partial u_{i}^{\prime}}{\partial x_{j}}}\right)^{2},
$$

a taxa de dissipação (ou de destruição) de energia cinética turbulenta. As duas equações diferenciais que governam essas duas variáveis turbulentas podem ser obtidas, levando-se em conta argumentos empíricos (McComb [94]), a partir das equações transientes de Navier-Stokes (Tennekes e Lumley [137], Harlow e Nakayama [52]). O conhecimento das variáveis $\kappa$ e $\varepsilon$ permite a avaliação da viscosidade turbulenta local $\nu_{t}$, a partir da qual as tensões turbulentas são estimadas.

O trabalho mais extensivo desenvolvido sobre o modelo $\kappa-\varepsilon$ foi realizado por Launder e Spalding [74, 75], cujo modelo proposto é usualmente referenciado na literatura como o modelo $\kappa-\varepsilon$ padrão ou modelo $\kappa-\varepsilon$ para número de Reynolds elevados.

No modelo $\kappa-\varepsilon$ padrão, as escalas características de comprimento e de velocidade da turbulência são, respectivamente, estimadas em função das grandezas médias $\kappa$ e $\varepsilon$ como

$$
l_{t} \approx \frac{\kappa^{3 / 2}}{\varepsilon} \quad \text { e } \quad u_{t} \approx \kappa^{1 / 2}
$$

A escala de tempo do movimento turbulento, $T_{t}$, é dada por

$$
T_{t}=\frac{l_{t}}{u_{t}}=\frac{\kappa}{\varepsilon}
$$

A viscosidade turbulenta, $\nu_{t}$, dada em (2.27) é obtida de (2.32) segundo a proposta de Prandtl-Komolgorov

$$
\nu_{t} \approx l_{t} u_{t}=C_{\mu} \kappa T_{t}
$$

em que $C_{\mu}$ é uma constante empírica.

As variáveis turbulentas $\kappa$ e $\varepsilon$ adimensionais são definidas pela transformação

$$
\bar{\kappa}=\frac{L \kappa}{\nu U} \quad \text { e } \quad \bar{\varepsilon}=\frac{L^{2} \varepsilon}{\nu U^{2}}
$$

Aplicando-se as hipóteses, as aproximações e adimensionalizações anteriormente discutidas, a formulação do modelo $\kappa-\varepsilon$ padrão na forma adimensional conservativa é modelado pelas 
equações

$$
\begin{gathered}
\frac{\partial u_{i}}{\partial x_{i}}=0 \\
\frac{\partial u_{i}}{\partial t}+\frac{\partial\left(u_{i} u_{j}\right)}{\partial x_{j}}=-\frac{\partial p_{e}}{\partial x_{i}}+\frac{1}{R e} \frac{\partial}{\partial x_{j}}\left(\frac{\partial u_{i}}{\partial x_{j}}\right)+\frac{1}{F r^{2}} g_{i}+\frac{1}{R e} \frac{\partial}{\partial x_{j}}\left(\nu_{t} D_{i j}\right), i=1,2,3, \\
\frac{\partial \kappa}{\partial t}+\frac{\partial\left(\kappa u_{j}\right)}{\partial x_{j}}=\frac{1}{R e} \frac{\partial}{\partial x_{j}}\left(\left(1+\frac{\nu_{t}}{\sigma_{\kappa}}\right) \frac{\partial \kappa}{\partial x_{j}}\right)+\nu_{t} D_{i j} \frac{\partial u_{i}}{\partial x_{j}}-\varepsilon \\
\frac{\partial \varepsilon}{\partial t}+\frac{\partial\left(\varepsilon u_{j}\right)}{\partial x_{j}}=\frac{1}{R e} \frac{\partial}{\partial x_{j}}\left(\left(1+\frac{\nu_{t}}{\sigma_{\varepsilon}}\right) \frac{\partial \varepsilon}{\partial x_{j}}\right)+\left(C_{1 \varepsilon} \nu_{t} D_{i j} \frac{\partial u_{i}}{\partial x_{j}}-C_{2 \varepsilon} \varepsilon\right) / T_{t} \\
T_{t}=\frac{\kappa}{\varepsilon} \\
\nu_{t}=C_{\mu} \kappa T_{t},
\end{gathered}
$$

em que $\sigma_{\kappa}$ e $\sigma_{\varepsilon}$, que aparecem em (2.38) e (2.39), são coeficientes de difusão turbulentos, e $C_{1 \varepsilon}$ e $C_{2 \varepsilon}$ em (2.39) são constantes empíricas. Essas constantes foram obtidas a partir da correlação de dados experimentais de diversos escoamentos turbulentos (Jones e Launder [65]), sendo dadas por

$$
C_{\mu}=0.09, \quad C_{1 \varepsilon}=1.44, \quad C_{2 \varepsilon}=1.92, \quad \sigma_{\kappa}=1.0, \quad \sigma_{\varepsilon}=1.3
$$

O modelo $\kappa-\varepsilon$, na forma geral adimensional e conservativa, também pode ser escrito em notação de Gibbs como

$$
\begin{gathered}
\nabla \cdot \mathbf{u}=0 \\
\frac{\partial \mathbf{u}}{\partial t}+\nabla \cdot(\mathbf{u u})=-\nabla p_{e}+\frac{1}{R e} \nabla^{2} \mathbf{u}+\frac{1}{F r^{2}} \mathbf{g}+\frac{1}{R e} \nabla \cdot\left(\nu_{t} \mathbf{D}\right) \\
\frac{\partial \kappa}{\partial t}+\nabla \cdot(\kappa \mathbf{u})=\frac{1}{R e} \nabla \cdot\left(\left(1+\frac{\nu_{t}}{\sigma_{\kappa}}\right) \nabla \kappa\right)+\mathbf{P}-\varepsilon \\
\frac{\partial \varepsilon}{\partial t}+\nabla \cdot(\varepsilon \mathbf{u})=\frac{1}{R e} \nabla \cdot\left(\left(1+\frac{\nu_{t}}{\sigma_{\varepsilon}}\right) \nabla \varepsilon\right)+\left(C_{1 \varepsilon} \mathbf{P}-C_{2 \varepsilon} \varepsilon\right) / T_{t} \\
T_{t}=\frac{\kappa}{\varepsilon} \\
\nu_{t}=C_{\mu} \kappa T_{t}
\end{gathered}
$$

em que

- $\mathbf{D}=(\nabla \mathbf{u})+(\nabla \mathbf{u})^{T} \quad$ é o tensor de deformações médio,

- $\mathbf{P}=\nu_{t}(\mathbf{D}: \nabla \mathbf{u}) \quad$ é o termo de produção de energia cinética turbulenta média.

No caso 3D, a forma expandida das equações do modelo $\kappa-\varepsilon$ padrão, utilizando-se coor- 
denadas cartesianas, tornam-se

$$
\begin{aligned}
& \frac{\partial u}{\partial x}+\frac{\partial v}{\partial y}+\frac{\partial w}{\partial z}=0 \\
& \frac{\partial u}{\partial t}=C O N V(u)-\frac{\partial p_{e}}{\partial x}+\frac{1}{R e}\left(\frac{\partial^{2} u}{\partial x^{2}}+\frac{\partial^{2} u}{\partial y^{2}}+\frac{\partial^{2} u}{\partial z^{2}}\right)+\frac{1}{F r^{2}} g_{x} \\
& +\frac{1}{R e}\left[2 \frac{\partial}{\partial x}\left(\nu_{t} \frac{\partial u}{\partial x}\right)+\frac{\partial}{\partial y}\left(\nu_{t}\left(\frac{\partial u}{\partial y}+\frac{\partial v}{\partial x}\right)\right)+\frac{\partial}{\partial z}\left(\nu_{t}\left(\frac{\partial u}{\partial z}+\frac{\partial w}{\partial x}\right)\right)\right], \\
& \frac{\partial v}{\partial t}=C O N V(v)-\frac{\partial p_{e}}{\partial y}+\frac{1}{R e}\left(\frac{\partial^{2} v}{\partial x^{2}}+\frac{\partial^{2} v}{\partial y^{2}}+\frac{\partial^{2} v}{\partial z^{2}}\right)+\frac{1}{F r^{2}} g_{y} \\
& +\frac{1}{R e}\left[2 \frac{\partial}{\partial y}\left(\nu_{t} \frac{\partial v}{\partial y}\right)+\frac{\partial}{\partial x}\left(\nu_{t}\left(\frac{\partial u}{\partial y}+\frac{\partial v}{\partial x}\right)\right)+\frac{\partial}{\partial z}\left(\nu_{t}\left(\frac{\partial v}{\partial z}+\frac{\partial w}{\partial y}\right)\right)\right], \\
& \frac{\partial w}{\partial t}=C O N V(w)-\frac{\partial p_{e}}{\partial z}+\frac{1}{R e}\left(\frac{\partial^{2} w}{\partial x^{2}}+\frac{\partial^{2} w}{\partial y^{2}}+\frac{\partial^{2} w}{\partial z^{2}}\right)+\frac{1}{F r^{2}} g_{z} \\
& +\frac{1}{R e}\left[2 \frac{\partial}{\partial z}\left(\nu_{t} \frac{\partial w}{\partial z}\right)+\frac{\partial}{\partial x}\left(\nu_{t}\left(\frac{\partial u}{\partial z}+\frac{\partial w}{\partial x}\right)\right)+\frac{\partial}{\partial y}\left(\nu_{t}\left(\frac{\partial v}{\partial z}+\frac{\partial w}{\partial y}\right)\right)\right], \\
& \frac{\partial \kappa}{\partial t}=C O N V(\kappa)+\frac{1}{R e}\left[\frac{\partial}{\partial x}\left(\left(1+\nu_{t} / \sigma_{\kappa}\right) \frac{\partial \kappa}{\partial x}\right)+\frac{\partial}{\partial y}\left(\left(1+\nu_{t} / \sigma_{\kappa}\right) \frac{\partial \kappa}{\partial y}\right)\right. \\
& \left.+\frac{\partial}{\partial z}\left(\left(1+\nu_{t} / \sigma_{\kappa}\right) \frac{\partial \kappa}{\partial z}\right)\right]+P-\varepsilon \\
& \frac{\partial \varepsilon}{\partial t}=C O N V(\varepsilon)+\frac{1}{R e}\left[\frac{\partial}{\partial x}\left(\left(1+\nu_{t} / \sigma_{\varepsilon}\right) \frac{\partial \varepsilon}{\partial x}\right)+\frac{\partial}{\partial y}\left(\left(1+\nu_{t} / \sigma_{\varepsilon}\right) \frac{\partial \varepsilon}{\partial y}\right)\right. \\
& \left.+\frac{\partial}{\partial z}\left(\left(1+\nu_{t} / \sigma_{\varepsilon}\right) \frac{\partial \kappa}{\partial z}\right)\right]+\left(C_{1 \varepsilon} P-C_{2 \varepsilon} \varepsilon\right) / T_{t} \\
& T_{t}=\frac{\kappa}{\varepsilon}, \\
& \nu_{t}=C_{\mu} \kappa T_{t} \\
& P=\nu_{t}\left[2\left(\frac{\partial u}{\partial x}\right)^{2}+2\left(\frac{\partial v}{\partial y}\right)^{2}+\left(\frac{\partial w}{\partial z}\right)^{2}+\left(\frac{\partial u}{\partial y}+\frac{\partial v}{\partial x}\right)^{2}\right. \\
& \left.+\left(\frac{\partial u}{\partial z}+\frac{\partial w}{\partial x}\right)^{2}+\left(\frac{\partial v}{\partial z}+\frac{\partial w}{\partial y}\right)^{2}\right] \text {. }
\end{aligned}
$$

Os termos advectivos $C O N V(\phi),(\phi=u, v, w, \kappa$ ou $\varepsilon)$, definidos em (2.49)-(2.53) são, respectivamente, representados como

$$
C O N V(u)=-\left(\frac{\partial(u u)}{\partial x}+\frac{\partial(u v)}{\partial y}+\frac{\partial(u w)}{\partial z}\right)
$$




$$
\begin{aligned}
& \operatorname{CONV}(v)=-\left(\frac{\partial(v u)}{\partial x}+\frac{\partial(v v)}{\partial y}+\frac{\partial(v w)}{\partial z}\right), \\
& \operatorname{CONV}(w)=-\left(\frac{\partial(w u)}{\partial x}+\frac{\partial(w v)}{\partial y}+\frac{\partial(w w)}{\partial z}\right), \\
& \operatorname{CONV}(\kappa)=-\left(\frac{\partial(\kappa u)}{\partial x}+\frac{\partial(\kappa v)}{\partial y}+\frac{\partial(\kappa w)}{\partial z}\right), \\
& \operatorname{CONV}(\varepsilon)=-\left(\frac{\partial(\varepsilon u)}{\partial x}+\frac{\partial(\varepsilon v)}{\partial y}+\frac{\partial(\varepsilon w)}{\partial z}\right) .
\end{aligned}
$$

Na seção 3.5 é apresentado o esquema "upwind" limitado de alta ordem, utilizado para discretizar os termos advectivos das equações (2.57)-(2.61).

Uma forma alternativa das equações do movimento que evita problemas numéricos nas vizinhas de descontinuidade, conhecida como completamente conservativa (Deville [30]), pode ser obtida introduzindo-se o vetor de vorticidade

$$
\omega=\nabla \times \mathbf{u}
$$

de maneira que

$$
\nabla^{2} \mathbf{u}=-\nabla \times \omega
$$

Utilizando o vetor vorticidade (2.62), as equações de conservação dadas por (2.49)-(2.53), são expressas na forma completamente conservativa por

$$
\begin{gathered}
\frac{\partial u}{\partial x}+\frac{\partial v}{\partial y}+\frac{\partial w}{\partial z}=0 \\
\frac{\partial u}{\partial t}=C O N V(u)-\frac{\partial p_{e}}{\partial x}+\frac{1}{R e}\left[\frac{\partial}{\partial y}\left(\frac{\partial u}{\partial y}-\frac{\partial v}{\partial x}\right)+\frac{\partial}{\partial z}\left(\frac{\partial u}{\partial z}-\frac{\partial w}{\partial x}\right)\right. \\
\left.+2 \frac{\partial}{\partial x}\left(\nu_{t} \frac{\partial u}{\partial x}\right)+\frac{\partial}{\partial y}\left(\nu_{t}\left(\frac{\partial u}{\partial y}+\frac{\partial v}{\partial x}\right)\right)+\frac{\partial}{\partial z}\left(\nu_{t}\left(\frac{\partial u}{\partial z}+\frac{\partial w}{\partial x}\right)\right)\right]+\frac{1}{F r^{2}} g_{x}, \\
\frac{\partial v}{\partial t}=C O N V(v)-\frac{\partial p_{e}}{\partial y}-\frac{1}{R e}\left[\frac{\partial}{\partial x}\left(\frac{\partial u}{\partial y}-\frac{\partial v}{\partial x}\right)-\frac{\partial}{\partial z}\left(\frac{\partial v}{\partial z}-\frac{\partial w}{\partial y}\right)\right. \\
\left.-2 \frac{\partial}{\partial y}\left(\nu_{t} \frac{\partial v}{\partial y}\right)-\frac{\partial}{\partial x}\left(\nu_{t}\left(\frac{\partial u}{\partial y}+\frac{\partial v}{\partial x}\right)\right)-\frac{\partial}{\partial z}\left(\nu_{t}\left(\frac{\partial v}{\partial z}+\frac{\partial w}{\partial y}\right)\right)\right]+\frac{1}{F r^{2}} g_{y},
\end{gathered}
$$




$$
\begin{aligned}
\frac{\partial w}{\partial t}= & C O N V(w)-\frac{\partial p_{e}}{\partial z}-\frac{1}{R e}\left[\frac{\partial}{\partial x}\left(\frac{\partial u}{\partial z}-\frac{\partial w}{\partial x}\right)+\frac{\partial}{\partial y}\left(\frac{\partial v}{\partial z}-\frac{\partial w}{\partial y}\right)\right. \\
& \left.-2 \frac{\partial}{\partial z}\left(\nu_{t} \frac{\partial w}{\partial z}\right)-\frac{\partial}{\partial x}\left(\nu_{t}\left(\frac{\partial u}{\partial z}+\frac{\partial w}{\partial x}\right)\right)-\frac{\partial}{\partial y}\left(\nu_{t}\left(\frac{\partial v}{\partial z}+\frac{\partial w}{\partial y}\right)\right)\right]+\frac{1}{F r^{2}} g_{z} \\
\frac{\partial \kappa}{\partial t}= & C O N V(\kappa)+\frac{1}{R e}\left[\frac{\partial}{\partial x}\left(\left(1+\nu_{t} / \sigma_{\kappa}\right) \frac{\partial \kappa}{\partial x}\right)+\frac{\partial}{\partial y}\left(\left(1+\nu_{t} / \sigma_{\kappa}\right) \frac{\partial \kappa}{\partial y}\right)\right. \\
& \left.+\frac{\partial}{\partial z}\left(\left(1+\nu_{t} / \sigma_{\kappa}\right) \frac{\partial \kappa}{\partial z}\right)\right]+P-\varepsilon \\
\frac{\partial \varepsilon}{\partial t}= & C O N V(\varepsilon)+\frac{1}{R e}\left[\frac{\partial}{\partial x}\left(\left(1+\nu_{t} / \sigma_{\varepsilon}\right) \frac{\partial \varepsilon}{\partial x}\right)+\frac{\partial}{\partial y}\left(\left(1+\nu_{t} / \sigma_{\varepsilon}\right) \frac{\partial \varepsilon}{\partial y}\right)\right. \\
& \left.+\frac{\partial}{\partial z}\left(\left(1+\nu_{t} / \sigma_{\varepsilon}\right) \frac{\partial \kappa}{\partial z}\right)\right]+\left(C_{1 \varepsilon} P-C_{2 \varepsilon} \varepsilon\right) / T_{t}
\end{aligned}
$$

Além do modelo $\kappa-\varepsilon$ padrão existem na literatura outras variações do modelo $\kappa-\varepsilon$ como por exemplo, o modelo $\kappa-\varepsilon$ KaLa ("Kato-Launder) proposta por Kato e Launder [68] e o modelo $\kappa-\varepsilon$ RNG ("Renormalization Group") baseado na teoria de renormalização (Yakhot e Orszag [160] e Yakhot et al. [161]).

O modelo $\kappa-\varepsilon$ KaLa é semelhante ao $\kappa-\varepsilon$ padrão, em que as equações de transporte para $\kappa$ e $\varepsilon$ são as mesmas, exceto o termo de produção de energia cinética turbulenta que é dado por

$$
P=C_{\mu} \varepsilon S \Omega
$$

em que

$$
S=\frac{\kappa}{\varepsilon} \sqrt{\frac{1}{2}\left(\frac{\partial u_{i}}{\partial x_{j}}+\frac{\partial u_{j}}{\partial x_{i}}\right)^{2}} \quad \text { e } \quad \Omega=\frac{\kappa}{\varepsilon} \sqrt{\frac{1}{2}\left(\frac{\partial u_{i}}{\partial x_{j}}-\frac{\partial u_{j}}{\partial x_{i}}\right)^{2}} .
$$

A quantidade $\Omega$ está relacionada com a rotação média do fluido. A viscosidade turbulenta, $\nu_{t}$, para o modelo $\kappa-\varepsilon$ KaLa é calculada da mesma forma do modelo $\kappa-\varepsilon$ padrão.

Yakhot e Orszag [160] prôpos uma outra variação do modelo $\kappa-\varepsilon$ utilizando a teoria de renormalização. O modelo $\kappa-\varepsilon$ RNG descreve a dinâmica do escoamento médio $u_{i}$, da energia cinética turbulenta $\kappa$ e da dissipação de energia $\varepsilon$. A teoria RNG também propõe expressões gerais para tratar escoamentos junto ao contorno rígido. Neste modelo, diferentemente da versão tradicional, é adotada uma abordagem numérica para a determinação das constantes e funções presentes nas equações de transporte.

Smith e Reynolds [123] identificaram vários problemas na derivação da equação de transporte $\varepsilon$ usando a teoria RNG. Várias melhorias foram realizadas originando uma nova versão do modelo $\kappa-\varepsilon$ RNG. Esse modelo tem se mostrado adequado à previsão de escoamentos em regiões internas de camada-limite (baixos números de Reynolds) sem a inclusão de termos de correção e em situações de linhas de correntes curvas, separação e estagnação. 
A formulação do modelo $\kappa-\varepsilon$ RNG, na forma adimensional e conservativa, é dada pelas seguintes equações de transporte

$$
\begin{gathered}
\frac{\partial \kappa}{\partial t}+\frac{\partial\left(\kappa u_{j}\right)}{\partial x_{j}}=\frac{1}{R e} \frac{\partial}{\partial x_{j}}\left(\left(1+\frac{\nu_{t}}{\sigma_{\kappa}}\right) \frac{\partial \kappa}{\partial x_{j}}\right)+P-\varepsilon \\
\frac{\partial \varepsilon}{\partial t}+\frac{\partial\left(\varepsilon u_{j}\right)}{\partial x_{j}}=\frac{1}{R e} \frac{\partial}{\partial x_{j}}\left(\left(1+\frac{\nu_{t}}{\sigma_{\varepsilon}}\right) \frac{\partial \varepsilon}{\partial x_{j}}\right)+\left(C_{1 \varepsilon} P-C_{2 \varepsilon} \varepsilon\right) / T_{t}-R \\
\nu_{t}=C_{\mu} \frac{\kappa^{2}}{\varepsilon}
\end{gathered}
$$

em que $R$ é termo da taxa de tensão dado por

$$
R=\frac{C_{\mu} s^{3}\left(1-\eta / \eta_{0}\right)}{1+\beta \eta^{3}}
$$

na qual

$$
\eta=s \frac{\kappa}{\varepsilon}
$$

$\operatorname{com} \eta_{0} \approx 4.38$ e $\beta \approx 0.015$ (derivado de forma que a constante de von Kármán $K$ se torna 0,41 ). O termo $R$ introduz um mecanismo único para descrever de uma forma geral, tão bem quanto balanços modificados temporariamente entre os termos de produção e dissipação na equação de $\varepsilon$.

A magnitude da taxa média de tensão $s$ é expressa por

$$
s=\sqrt{2 s_{i j} s_{i j}}
$$

em que o tensor da taxa média de tensão $s_{i j}$ é dado por

$$
s_{i j}=\frac{1}{2}\left(\frac{\partial u_{i}}{\partial x_{j}}+\frac{\partial u_{j}}{\partial x_{i}}\right)
$$

O termo de produção de energia cinética turbulenta, $P$, é calculado da mesma forma como no modelo $\kappa-\varepsilon$ padrão, ou seja,

$$
P=\nu_{t}\left(\frac{\partial u_{i}}{\partial x_{j}}+\frac{\partial u_{j}}{\partial x_{i}}\right) \frac{\partial u_{i}}{\partial x_{j}}
$$

Da teoria RNG de Yakhot e Orszag [160], as constantes são dadas agora por

$$
C_{\mu}=0.085, \quad C_{1 \varepsilon}=1.42-\frac{\eta\left(1-\eta / \eta_{0}\right)}{1+\beta \eta^{3}}, \quad C_{2 \varepsilon}=1.68, \quad \sigma_{\kappa}=\sigma_{\varepsilon}=0.7179
$$




\subsubsection{Condições Iniciais e de Contorno}

Para resolver as equações (2.63)-(2.68) é necessário impor condições iniciais e de contorno adequadas. A escolha dessas condições é muito importante para a formulação dos problemas, pois o comportamento físico da solução depende da escolha dessas condições.

As condições iniciais para as variáveis médias de velocidade e pressão são prescritas no início dos cálculos (Tomé e McKee [143]). No caso do modelo $\kappa-\varepsilon$ é necessário especificar valores para as variáveis turbulentas $\kappa$ e $\varepsilon$ nas entradas. É difícil especificar as condições iniciais para essas variáveis, uma vez que elas devem estar em concordância com a física do problema. Nos problemas envolvendo superfícies livres, esse tipo de informação nem sempre está disponível. Sendo assim, torna-se necessário fazer uma suposição baseada em uma certa experiência sobre a turbulência na entrada, da mesma forma como está feito em diversos trabalhos na literatura, como por exemplo, nos estudos realizados por Manouzi e Fortin [92], Robinson e Hassan [113], entre muitos outros. Neste trabalho, as condições iniciais para as variáveis turbulentas são definidas em função de uma intensidade $I$ de turbulência e da velocidade característica $U$ associada às grandes escalas. Estas propriedades são mais intuitivas de compreender e podem ser relacionadas mais facilmente às características físicas do problema.

Uma vez que uma intensidade apropriada de turbulência e uma velocidade característica são estimadas, as variáveis do modelo de turbulência podem ser calculadas. Dessa forma, para a energia cinética turbulenta, $\kappa$, assume-se que

$$
\kappa=\kappa(I, U)=I U^{2}
$$

e a dissipação de energia, $\varepsilon$, pode ser calculada como

$$
\varepsilon=\varepsilon\left(\kappa, l_{t}\right)=\frac{\kappa^{3 / 2}}{l_{t}}
$$

em que $l_{t}$ é a escala de comprimento, que geralmente, é definida por $l_{t}=\beta L$ (Elkaim et al. [34], Shuja e Yilbas [121]), na qual $L$ é uma escala de comprimento turbulenta e $\beta$ uma constante, usualmente, da ordem de $10^{-2}$, dependendo do problema. Neste estudo, o valor de $\beta$ utilizado nas implementações foi de $3 \times 10^{-2}$. A escala de comprimento turbulento, $L$, é uma quantidade física representativa das grandes escalas que contém os maiores níveis de energia em um escoamento turbulento.

No código Freeflow 3D, utilizando-se as transformações (2.13) e (2.35), tem-se que as condições iniciais adimensionais para as variáveis turbulentas são implementadas explicitamente como

$$
\kappa=I R e \quad \text { e } \quad \varepsilon=\frac{1}{\beta}\left(\frac{\kappa^{3}}{R e}\right)^{1 / 2} .
$$

A viscosidade turbulenta, $\nu_{t}$, é então, dada pela representação de Prandtl-Komolgorov (2.34) e 
a intensidade de turbulência é fixada como sendo $I=0.080$.

As condições de contorno são requeridas para completar o problema que se deseja resolver. Logo, é necessário ter alguma informação física das variáveis dependentes na fronteira da região limitando o escoamento. Nos escoamentos incompressíveis de interesse nesse trabalho, os seguintes tipos de condições de contorno foram adotados:

(1) Condições na entrada e saída de fluido do domínio: É necessário o conhecimento do escoamento nos contornos da entrada e saída do domínio.

- Entrada de fluido ou injetor ("Inflow"): Condições de contorno prescritas, ou seja,

$$
\mathbf{u} \cdot \mathbf{n}=U, \quad \text { e } \quad \mathbf{u} \cdot \mathbf{m}_{1}=\mathbf{u} \cdot \mathbf{m}_{\mathbf{2}}=0,
$$

na qual $\mathbf{n}$ é vetor normal unitário e $\mathbf{m}_{\mathbf{1}}$ e $\mathbf{m}_{\mathbf{2}}$ são vetores tangenciais ao contorno.

- Saída de fluido ou ejetor ("Outflow"): Condições de contorno do tipo Neumann homogênea.

As condições de contorno na entrada para a energia cinética turbulenta, $\kappa$, e para a dissipação de energia, $\varepsilon$, são impostas, consistentemente com as condições iniciais definidas em (2.77). Na saída de fluido, essas variáveis turbulentas são calculadas pela aplicação da condição de Neumann homogênea, ou seja,

$$
\frac{\partial \kappa}{\partial n}=0 \quad \text { e } \quad \frac{\partial \varepsilon}{\partial n}=0
$$

\section{(2) Condição sobre uma superfície rígida:}

- Condição de contorno sem deslizamento ("no-slip"): Esta condição significa que o fluido deve ser fixado à superfície, ou seja, define-se

$$
\mathbf{u}=0 .
$$

- Condição de contorno sem aderência ou de simetria ("free-slip"): É utilizada quando há fronteiras de simetria, ou quando os efeitos da condição de contorno sem deslizamento não são desejáveis. Neste caso define-se

$$
\mathbf{u} \cdot \mathbf{n}=0
$$

e condições de contorno do tipo Neumann homogênea para a velocidade tangencial.

Próximo ao contorno rígido aplicam-se, neste trabalho, as equações de quantidade de movimento normalmente, e para as variáveis turbulentas, $\kappa \mathrm{e} \varepsilon$ aplica-se uma modificação das leis de parede (ver Sondak e Pletcher [125] e Durbin [32]). Em resumo, a viscosidade turbulenta é calculada usando-se a função de amortecimento de van Driest [147] dada por

$$
\nu_{t}=K u^{*} y\left(1-\exp \left(\frac{-y^{+}}{A}\right)\right)^{2}
$$


e

$$
y^{+}=\frac{u^{*} y}{\nu},
$$

na qual $A \approx 26, K=0.41$ é a constante de von Kármán (ver Patel [107]), $y^{+}$é o número de Reynolds local e $u^{*}$ é a velocidade de atrito (escala de velocidade). As variáveis turbulentas nessa região são calculadas de duas maneiras (nas subcamadas turbulenta e viscosa).

Na subcamada turbulenta, as condições de contorno para as variáveis turbulentas $\kappa$ e $\varepsilon$ na forma adimensional são implementadas como (ver detalhes em Anderson et al [5], Ferziger e Perić [45])

$$
\kappa=R e \frac{u^{* 2}}{\sqrt{C_{\mu}}} \quad \text { e } \quad \varepsilon=R e \frac{u^{* 3}}{K y} .
$$

Na subcamada viscosa, a energia cinética turbulenta $\kappa$ adimensionalizada é implementada no código Freeflow 3D utilizando-se a correção de Sondak e Pletcher [125]

$$
\kappa=R e \frac{u^{* 2}}{\sqrt{C_{\mu}}}\left(\frac{y^{+}}{y_{c}^{+}}\right)^{2},
$$

em que $y^{+}$é dado por (2.83) e $y_{c}^{+}$, número de Reynolds local crítico, é a solução da equação não linear definida pela intersecção das leis

$$
u^{+}=R e y u^{*}
$$

e

$$
\ln \left(\text { EReyu }^{*}\right)-K \frac{u}{u^{*}}=0,
$$

isto é,

$$
\ln \left(E y_{c}^{+}\right)-K y_{c}^{+}=0
$$

em que $E=\exp (K B)$ e $u$ é a magnitude da velocidade tangencial resultante à distância $y$ da parede. Para escoamentos newtonianos sobre superfícies suaves, dados experimentais permitem concluir que $B$ está entre 5.0-5.2 (Bradshaw [14]).

As relações (2.86) e (2.87) são conhecidas na literatura como leis de paredes clássicas. É importante ressaltar que essas leis são restritas a determinados valores do número de Reynolds local $y^{+}$. De acordo com Rodi [114], McComb [94], MacDonough [89] e outros, a lei (2.86) é válida até $y^{+} \approx 5.0$ e (2.87) é aplicável somente na região onde $30<y^{+}<300$ (ver Figura 2.2).

O valor da dissipação de energia $\varepsilon$, em forma adimensional, na subcamada viscosa é implementado impondo-se a escala de comprimento $l^{*}$ proposta por Norris e Reynolds [100]

$$
l^{*}=\frac{K C_{\mu}^{-\frac{3}{4}} y}{1+5.3 / R e_{t}},
$$




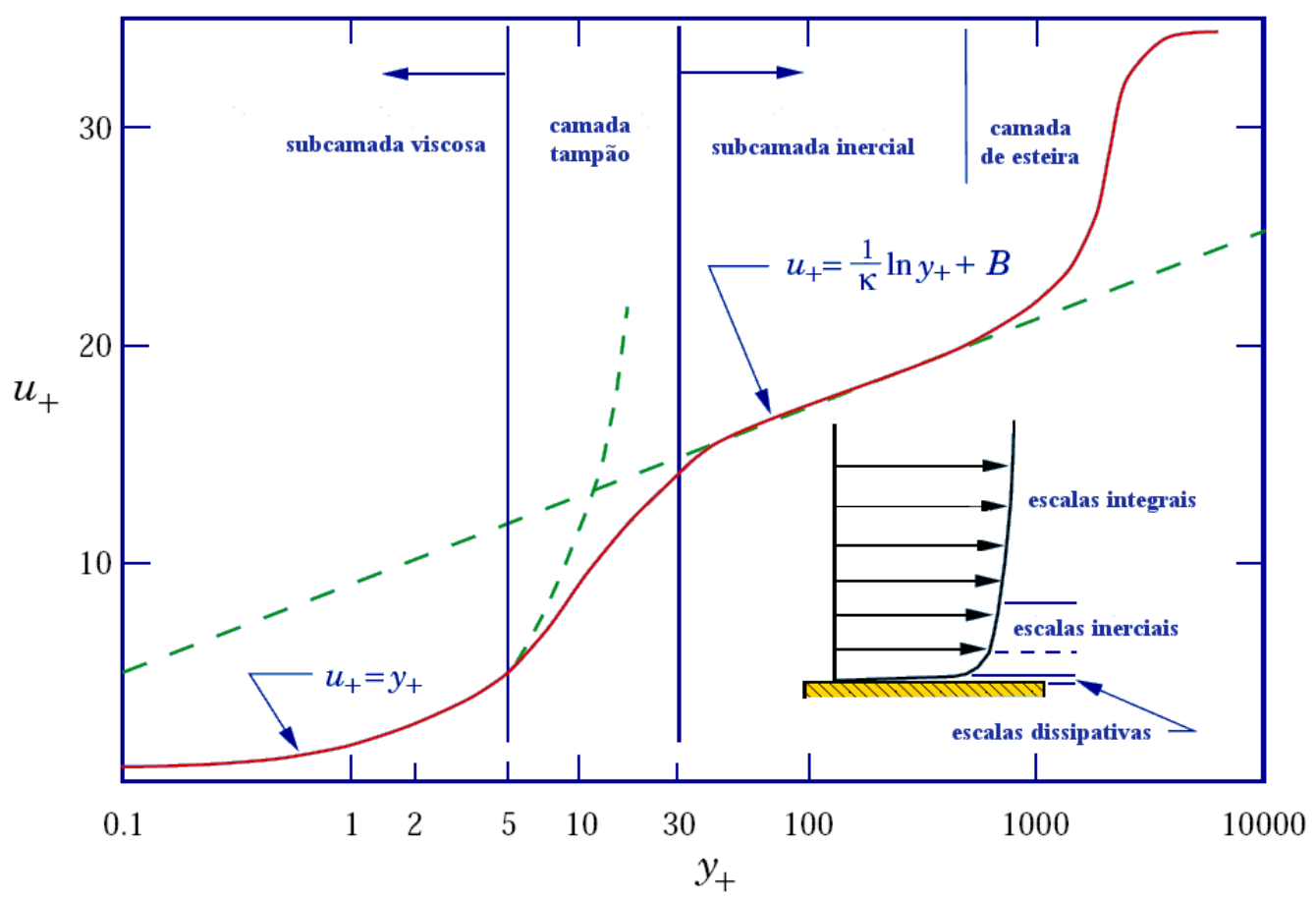

Figura 2.2: Regiões diferentes de um escoamento próximo à uma parede. Adaptado de MacDonough (2004).

em que

$$
R e_{t}=y \sqrt{R e \kappa}
$$

Logo, a variável $\varepsilon$ é calculada pela expressão

$$
\varepsilon=\sqrt{\frac{1}{R e}} \frac{\kappa^{\frac{3}{2}}}{l^{*}}
$$

As velocidades fictícias são implementadas forçando-se, nas equações médias do movimento, a tensão cisalhante adimensional total $\tau_{\omega}$ dada por (ver Wilcox [159])

$$
\left.\left(\frac{1}{R e}\left(1+\nu_{t}\right)\left|\frac{\partial V}{\partial n}\right|\right)\right|_{\text {parede }} \approx u^{* 2}=\tau_{\omega},
$$

em que $V$ e $n$ são, respectivamente, as velocidade tangenciais e a direção normal ao contorno rígido. O lado esquerdo da equação (2.92) é avaliado no contorno rígido, e os valores de $V$ fora do domínio de solução são obtidos discretizando-se a derivada por diferenças centrais, levando-se em conta que a tensão efetiva possui sinal oposto ao da velocidade tangencial (mais detalhes podem ser encontrados em Ferreira et al. [42]).

(3) Condição sobre uma superfície livre: Na superfície livre é necessário impor condições nos campos de velocidade e pressão. Para o caso 3D, essas condições, na ausência de tensão 
superficial são escritas como (ver detalhes em Batchelor [11])

$$
\begin{aligned}
\mathbf{n} \cdot(\boldsymbol{\sigma} \cdot \mathbf{n}) & =p_{\text {ext }} \\
\mathbf{m}_{\mathbf{1}} \cdot(\boldsymbol{\sigma} \cdot \mathbf{n}) & =0 \\
\mathbf{m}_{\mathbf{2}} \cdot(\boldsymbol{\sigma} \cdot \mathbf{n}) & =0
\end{aligned}
$$

em que $p_{\text {ext }}$ é a pressão (atmosférica) externa, $\mathbf{m}_{\mathbf{1}}=\left(m_{1 x}, m_{1 y}, m_{1 z}\right)$ e $\mathbf{m}_{\mathbf{2}}=\left(m_{2 x}, m_{2 y}, m_{2 z}\right)$ são os vetores tangentes à superfície livre, $\mathbf{n}=\left(n_{x}, n_{y}, n_{z}\right)$ é o vetor unitário normal externo à superfície e $\boldsymbol{\sigma}$ é o tensor de tensões total dado por

$$
\boldsymbol{\sigma}=-p \mathbf{I}+\mu \mathbf{D}
$$

na qual $p$ é a pressão, I é o tensor identidade, e $\mu$ o coeficiente de viscosidade dinâmica.

Assumindo-se que $p_{\text {ext }}=0$ na equação (2.93) e substituindo-se o tensor de tensões dado por (2.96) nas equações (2.93)-(2.95) e utilizando-se as mudanças de variáveis (2.13), para adimensionalização, obtêm-se as seguintes equações

$$
\begin{gathered}
p-\frac{2}{R e}\left[\frac{\partial u}{\partial x} n_{x}^{2}+\frac{\partial v}{\partial y} n_{y}^{2}+\frac{\partial w}{\partial z} n_{z}^{2}+\left(\frac{\partial u}{\partial y}+\frac{\partial v}{\partial x}\right) n_{x} n_{y}\right. \\
\left.+\left(\frac{\partial u}{\partial z}+\frac{\partial w}{\partial x}\right) n_{x} n_{z}+\left(\frac{\partial v}{\partial z}+\frac{\partial w}{\partial y}\right) n_{y} n_{z}\right]=0 \\
\frac{1}{R e}\left[2 \frac{\partial u}{\partial x} m_{1 x} n_{x}+2 \frac{\partial v}{\partial y} m_{1 y} n_{y}+2 \frac{\partial w}{\partial z} m_{1 z} n_{z}+\left(\frac{\partial u}{\partial y}+\frac{\partial v}{\partial x}\right)\left(m_{1 x} n_{y}+m_{1 y} n_{x}\right)\right. \\
\left.+\left(\frac{\partial u}{\partial z}+\frac{\partial w}{\partial x}\right)\left(m_{1 x} n_{z}+m_{1 z} n_{x}\right)+\left(\frac{\partial v}{\partial z}+\frac{\partial w}{\partial y}\right)\left(m_{1 y} n_{z}+m_{1 z} n_{y}\right)\right]=0
\end{gathered}
$$

e

$$
\begin{aligned}
\frac{1}{R e} & {\left[2 \frac{\partial u}{\partial x} m_{2 x} n_{x}+2 \frac{\partial v}{\partial y} m_{2 y} n_{y}+2 \frac{\partial w}{\partial z} m_{2 z} n_{z}+\left(\frac{\partial u}{\partial y}+\frac{\partial v}{\partial x}\right)\left(m_{2 x} n_{y}+m_{2 y} n_{x}\right)\right.} \\
& \left.+\left(\frac{\partial u}{\partial z}+\frac{\partial w}{\partial x}\right)\left(m_{2 x} n_{z}+m_{2 z} n_{x}\right)+\left(\frac{\partial v}{\partial z}+\frac{\partial w}{\partial y}\right)\left(m_{2 y} n_{z}+m_{2 z} n_{y}\right)\right]=0 .
\end{aligned}
$$

Para o caso turbulento, substituindo-se o tensor de tensões dado por

$$
\boldsymbol{\sigma}=(-p \delta+\nu \mathbf{D})+\left(-p_{t} \delta+\nu_{t} \mathbf{D}\right)
$$

nas equações (2.93)-(2.95) e utilizando-se as mudanças de variáveis (2.13) e (2.28), obtêm-se as 
seguintes condições de contorno na superfície livre

$$
\begin{gathered}
p_{e}-\frac{2}{R e}\left(1+\nu_{t}\right)\left[\frac{\partial u}{\partial x} n_{x}^{2}+\frac{\partial v}{\partial y} n_{y}^{2}+\frac{\partial w}{\partial x} n_{z}^{2}+\left(\frac{\partial u}{\partial y}+\frac{\partial v}{\partial x}\right) n_{x} n_{y}\right. \\
\left.+\left(\frac{\partial u}{\partial z}+\frac{\partial w}{\partial x}\right) n_{x} n_{z}+\left(\frac{\partial v}{\partial z}+\frac{\partial w}{\partial y}\right) n_{y} n_{z}\right]=0 \\
\frac{1}{R e}\left(1+\nu_{t}\right)\left[2 \frac{\partial u}{\partial x} m_{1 x} n_{x}+2 \frac{\partial v}{\partial y} m_{1 y} n_{y}+2 \frac{\partial w}{\partial z} m_{1 z} n_{z}+\left(\frac{\partial u}{\partial y}+\frac{\partial v}{\partial x}\right)\left(m_{1 x} n_{y}+m_{1 y} n_{x}\right)\right. \\
\left.+\left(\frac{\partial u}{\partial z}+\frac{\partial w}{\partial x}\right)\left(m_{1 x} n_{z}+m_{1 z} n_{x}\right)+\left(\frac{\partial v}{\partial z}+\frac{\partial w}{\partial y}\right)\left(m_{1 y} n_{z}+m_{1 z} n_{y}\right)\right]=0
\end{gathered}
$$

$\mathrm{e}$

$$
\begin{aligned}
\frac{1}{R e}(1 & \left.+\nu_{t}\right)\left[2 \frac{\partial u}{\partial x} m_{2 x} n_{x}+2 \frac{\partial v}{\partial y} m_{2 y} n_{y}+2 \frac{\partial w}{\partial z} m_{2 z} n_{z}+\left(\frac{\partial u}{\partial y}+\frac{\partial v}{\partial x}\right)\left(m_{2 x} n_{y}+m_{2 y} n_{x}\right)\right. \\
& \left.+\left(\frac{\partial u}{\partial z}+\frac{\partial w}{\partial x}\right)\left(m_{2 x} n_{z}+m_{2 z} n_{x}\right)+\left(\frac{\partial v}{\partial z}+\frac{\partial w}{\partial y}\right)\left(m_{2 y} n_{z}+m_{2 z} n_{y}\right)\right]=0 .
\end{aligned}
$$

A pressão efetiva na superfície livre é determinada pela equação (2.101) e, desde que $\nu_{t}>0$, a velocidade na interface é dada por (2.102) e (2.103). As equações (2.101)-(2.103) são aproximadas localmente por diferenças finitas, considerando-se as diferentes orientações da superfície livre definidas em Tomé e McKee [143] e Tomé et al. [140]. As versões discretas dessas equações são obtidas aproximando-se ambas as equações em pontos específicos da superfície e exigindo-se conservação de massa.

As condições de contorno para as variáveis turbulentas $\kappa$ e $\varepsilon$ na superfície livre do fluido são as mais difíceis de serem especificadas, devido à complexidade da dinâmica da turbulência próximo à interface. Não se sabe ainda, por exemplo, como a turbulência interage com a tensão superficial e, por conseguinte, é difícil estimar a distribuição de $\kappa$ sobre uma superfície irregular em movimento. Assim, como uma primeira aproximação, admite-se que a superfície livre é localmente planar e que o movimento ao acaso do fluido não causa descontinuidades na fronteira. Em resumo, as variáveis turbulentas na superfície livre são determinadas por

$$
\frac{\partial \kappa}{\partial n}=0 \quad \text { e } \quad \frac{\partial \varepsilon}{\partial n}=0
$$

em que as derivadas parciais em (2.104) são aproximadas localmente por diferenças avançadas ou atrasadas de primeira ordem. 


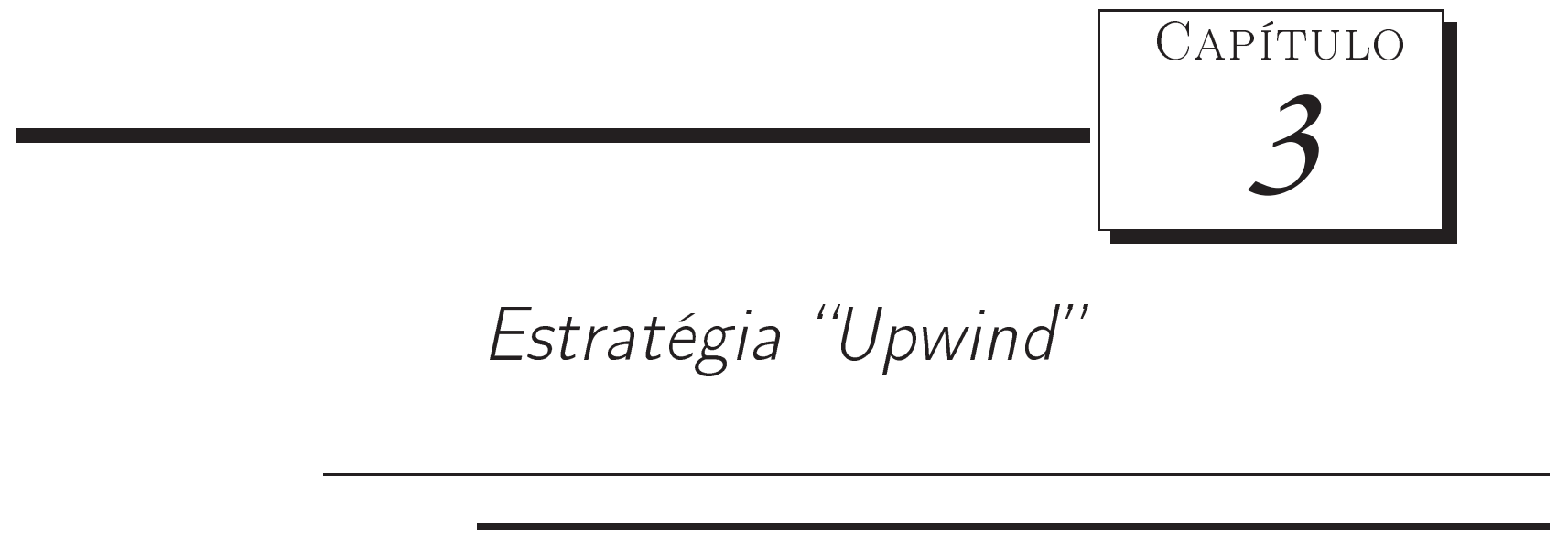

Neste capítulo, é apresentado a estratégia "upwind" ADBQUICKEST (de ordem maior ou igual a 2) adotada neste trabalho para aproximar os termos advectivos lineares e não lineares. Discutem-se as propriedades físicas e os aspectos teóricos fundamentais dos termos advectivos utilizando-se os conceitos de variáveis normalizadas (NV) de Leonard [80] e o critério de limitação (CBC) de Gaskell e Lau [47]. Discute-se, também, as restrições TVD de Harten $[54,55]$.

\subsection{Aproximações para os termos advectivos}

Nesta seção apresentam-se as aproximações para os termos advectivos que estão implementadas nos códigos Freeflow 2D e 3D utilizando um novo esquema "upwind" de alta ordem proposto por Kaibara et al. [66].

Os termos advectivos das equações (2.64)-(2.68) são os principais causadores de dificuldades numéricas nas simulações e responsáveis por fenômenos complexos no escoamento. Dessa forma, a aproximação desses termos merece atenção especial. Vale ressaltar que a estabilidade, exatidão e a limitação da solução obtida na simulação numérica depende do esquema numérico adotado para aproximar os termos advectivos.

Durante a apresentação dos termos advectivos e do esquema advectivo serão utilizadas as seguintes notações para as posições dos nós computacionais, com respeito a face $f$ e o sinal da velocidade advectiva $\mathbf{V}_{\mathbf{f}}$ na face $f$ : o a jusante $\mathbf{D}$ ("Downstream"), o a montante $\mathbf{U}$ ("Upstream") e o remoto a montante $\mathbf{R}$ ("Remote-upstream"), que estão ilustrados na Figura 3.1. De maneira análoga, usa-se as mesmas notações quando se tratar de uma outra face $g$ (ver Figura 3.2).

A variável convectada a ser aproximada na face $f$ da célula computacional é denotada por $\phi_{f}$ e na face $g$ por $\phi_{g}$, em que $\phi$ representa, no caso de problemas de escoamentos de fluidos, 
uma das variáveis $u, v, w, \kappa$ ou $\varepsilon$.
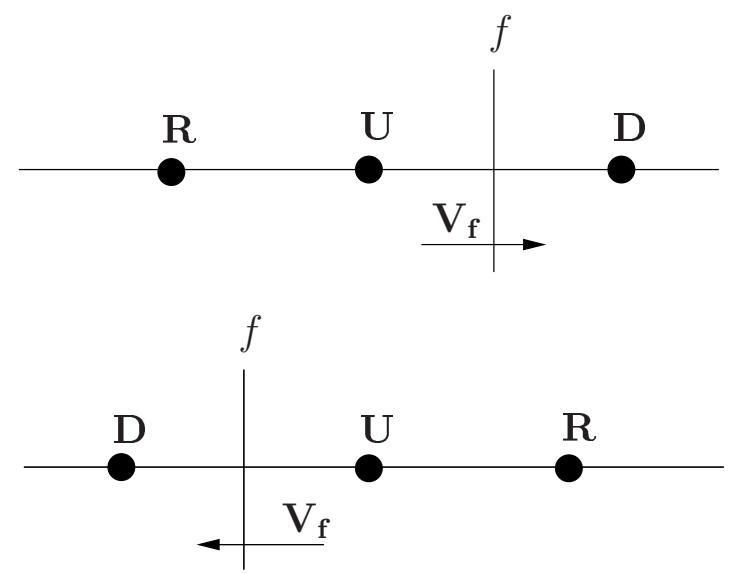

Figura 3.1: Posições dos nós $\mathbf{D}, \mathbf{U}$ e $\mathbf{R}$ conforme o sinal da variável convectada $\phi$ na face $f$, $\phi_{f}$.

Considere os termos advectivos dados pelas expressões (2.57)-(2.61) definidos em um ponto particular $\mathcal{P}$ do volume de controle (ver Figura 3.2), dado por

$$
\left.\operatorname{CONV}(\phi)\right|_{\mathcal{P}}=-\left(\left.\frac{\partial(\phi u)}{\partial x}\right|_{\mathcal{P}}+\left.\frac{\partial(\phi v)}{\partial y}\right|_{\mathcal{P}}+\left.\frac{\partial(\phi w)}{\partial z}\right|_{\mathcal{P}}\right)
$$

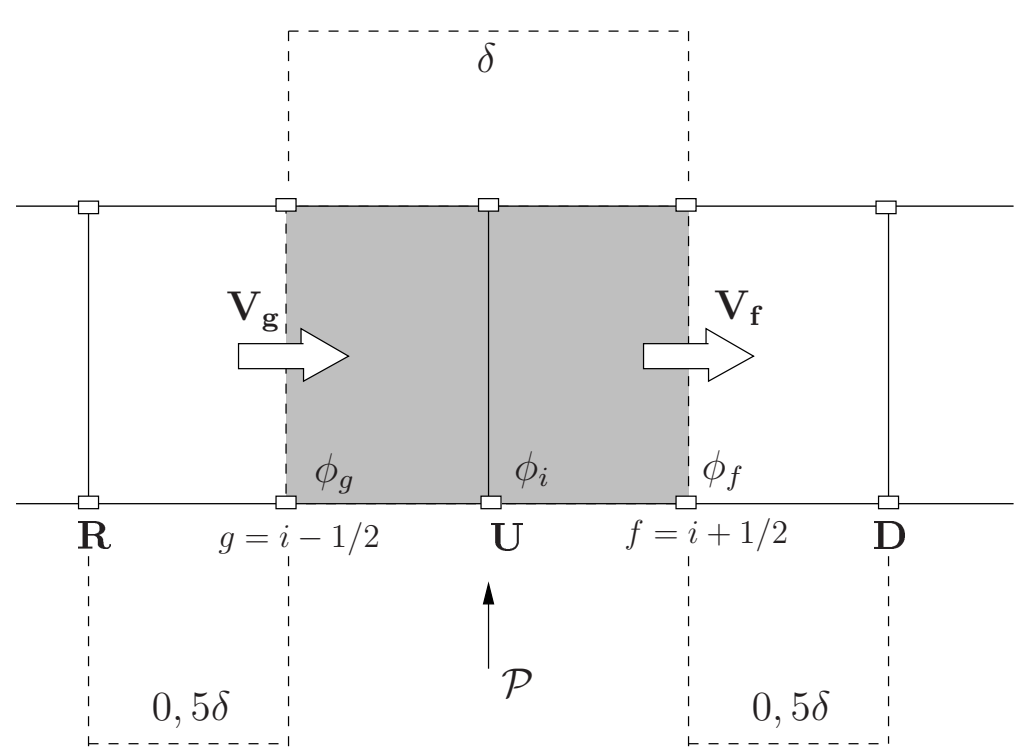

Figura 3.2: Representação esquemática para se obter aproximações dos termos advectivos, mostrando: $\mathrm{O}$ ponto $\mathcal{P}$ de discretização e seus vizinhos, as faces envolvidas $f$ e $g$ para a aproximação e a direção das velocidades $\mathbf{V}_{\mathbf{f}}$ e $\mathbf{V}_{\mathbf{g}}$ de advecção nas faces $f$ e $g$.

Por simplicidade, considera-se apenas a última derivada em (3.1), sem levar em conta o 
sinal. Assim, essa derivada é aproximada em $\mathcal{P}$ (ver Figura 3.2) pela diferença

$$
\left.\frac{\partial(\phi w)}{\partial z}\right|_{\mathcal{P}} \approx \frac{\left.(\phi w)\right|_{f}-\left.(\phi w)\right|_{g}}{\delta z}=\frac{\phi_{f} w_{f}-\phi_{g} w_{g}}{\delta z}
$$

em que $w_{f}$ e $w_{g}$ são estimados usando-se média e os valores vizinhos $\mathbf{U}$ e $\mathbf{D}$ para $f$, e $\mathbf{U}$ e $\mathbf{R}$ para $g$, ou seja,

$$
w_{f}=\frac{w_{D}+w_{U}}{2} \quad \text { e } \quad w_{g}=\frac{w_{R}+w_{U}}{2} .
$$

A aproximação para as demais derivadas é feita de forma análoga.

A maneira de como aproximar os valores de $\phi_{f}$ e $\phi_{g}$ em (3.2) é de fundamental importância para a estabilidade e a precisão das soluções numéricas. Por exemplo, a técnica de diferenças centrais aproxima $\phi_{f}$ pela média de $\phi_{U}$ e $\phi_{D}$, obtendo segunda ordem de precisão. Nesse caso, o fenômeno de dispersão associado a essa aproximação pode proporcionar oscilações na solução e instabilidade numérica, ocasionando uma solução não-limitada (Patankar [106], Hirsch [59]). Para se obter soluções limitadas, é necessário embutir informações físicas do escoamento nas aproximações numéricas. O tratamento "upwind", por exemplo, determina $\phi_{f}$ utilizando a direção de velocidade de advecção $\mathbf{V}_{\mathbf{f}}$ na face $f$. Nesse caso, soluções limitadas são garantidas pelo critério de estabilidade para sistemas de equações lineares (Patankar [106]). Mas se a aproximação "upwind" envolve esquemas de primeira ordem, a introdução implícita de viscosidade numérica (dissipação numérica) pode violar a ordem de precisão global da discretização e distorcer severamente a solução numérica.

\subsection{Restrições TVD}

A literatura é bastante ampla no tratamento desse tipo de método. Segue uma apresentação simplificada conforme, Darwish [27], tratando apenas de alguns aspectos básicos dos esquemas TVD. Considera-se o modelo escalar mais simples dado por

$$
\frac{\partial \phi}{\partial t}+a \frac{\partial \phi}{\partial x}=0
$$

com $a$ constante, cuja solução é aproximada pelo esquema conservativo

$$
\phi_{i}^{n+1}=\phi_{i}^{n}-\theta\left(\phi_{i+1 / 2}^{n}-\phi_{i-1 / 2}^{n}\right)
$$

em que $\theta=a \frac{\delta t}{\delta x}$ é o número de Courant e $\phi_{i+1 / 2}^{n}$ é uma aproximação da propriedade $\phi$ na face da célula $\left[x_{i-1 / 2}, x_{i+1 / 2}\right]$ cujo centro é $x_{i}$.

Dada uma sequência de aproximações discreta $\phi(t)=\left\{\phi_{i}(t)\right\}_{i \in \mathbb{Z}}$, uma função TV ("Total 
Variation") da solução discreta no tempo $t$ é definida por

$$
T V(\phi(t))=\sum_{i \in \mathbb{Z}}\left|\phi_{i+1}(t)-\phi_{i}(t)\right|
$$

Formalmente, considera-se o esquema de diferença explícito envolvendo $(2 k+1)$ pontos da forma

$$
\phi_{i}^{n+1}=H\left(\phi_{i-k}^{n}, \cdots, \phi_{i+k}^{n}\right), \quad \forall n \geq 0 \quad i \in \mathbb{Z},
$$

na qual $H: \mathbb{R}^{2 k+1} \longrightarrow \mathbb{R}$ é uma função contínua e $\phi_{i}^{n}$ denota uma aproximação para a solução exata $\phi$ num ponto da malha uniforme $\left(x_{i}, t_{n}\right)$, sendo $x_{i}=i \delta x, t_{n}=n \delta t$, em que $\delta x$ e $\delta t$ são os passos espaciais e temporais, respectivamente. O esquema é chamado de TVD ("Total Variation Diminishing") se satisfaz a seguinte desigualdade

$$
T V\left(\phi^{n+1}\right) \leq T V\left(\phi^{n}\right)
$$

Considere, agora, o esquema na forma

$$
\phi_{i}^{n+1}=\phi_{i}^{n}+C_{i+1 / 2}\left(\phi_{i+1}^{n}-\phi_{i}^{n}\right)-D_{i-1 / 2}\left(\phi_{i}^{n}-\phi_{i-1}^{n}\right)
$$

em que os coeficientes $C_{i+1 / 2}$ e $D_{i-1 / 2}$ dependem das $2 k$ variáveis $\phi_{i-k+1}, \ldots, \phi_{i+k}$. Harten [54] mostrou que se esses coeficientes satisfazem a seguintes restrições para todo valor de $i$,

$$
\begin{aligned}
C_{i+1 / 2} & \geq 0 \\
D_{i-1 / 2} & \geq 0 \\
C_{i+1 / 2}+D_{i-1 / 2} & \leq 1
\end{aligned}
$$

então o esquema é TVD.

Esquemas TVD, isto é, esquemas que satisfazem a restrições TVD, têm características atrativas. As soluções obtidas por esses esquemas são bem resolvidas, convergentes e não apresentam oscilações espúrias na solução numérica.

\subsection{Variáveis Normalizadas}

Para se obter esquemas capazes de resolver gradientes elevados e, ao mesmo tempo, manter estabilidade nas soluções numéricas, Leonard [80] e Gaskell e Lau [47] propuseram o conceito de variáveis normalizadas, também conhecida por NVF e o critério CBC, os quais, diferentemente do tratamento TVD, não garantem convergência, mas tratam o problema de estabilidade de uma maneira mais adequada.

Considere $\phi$ a variação de um escalar na direção normal a umas das faces $f$ ou $g$. A variável 
normalizada de Leonard é, então, definida por

$$
\hat{\phi}_{()}=\frac{\phi_{()}-\phi_{R}}{\phi_{D}-\phi_{R}}
$$

em que $\phi_{D}$ e $\phi_{R}$ são, respectivamente, os valores não-normalizados da propriedade $\phi$ nos pontos D e R. A partir da definição de (3.6), qualquer esquema de advecção que utilize somente os valores de $\phi$ nos pontos $\mathbf{D}, \mathbf{U}$ e $\mathbf{R}$ para avaliar $\phi_{f}$ pode ser representado na forma funcional

$$
\hat{\phi}_{f}=\hat{\phi}_{f}\left(\hat{\phi}_{U}\right)
$$

em que

$$
\hat{\phi}_{f}=\frac{\phi_{f}-\phi_{R}}{\phi_{D}-\phi_{R}} \quad \text { e } \quad \hat{\phi}_{U}=\frac{\phi_{U}-\phi_{R}}{\phi_{D}-\phi_{R}}
$$

Sem perda de generalidade, admite-se que o fluxo na face $f$ esteja na direção e no sentido do eixo coordenado $z$, isto é, $\mathbf{V}_{\mathbf{f}}>\mathbf{0}$. A Figura 3.3 ilustra, no caso unidimensional, a variação da variável transportada nas vizinhanças da face $f$.

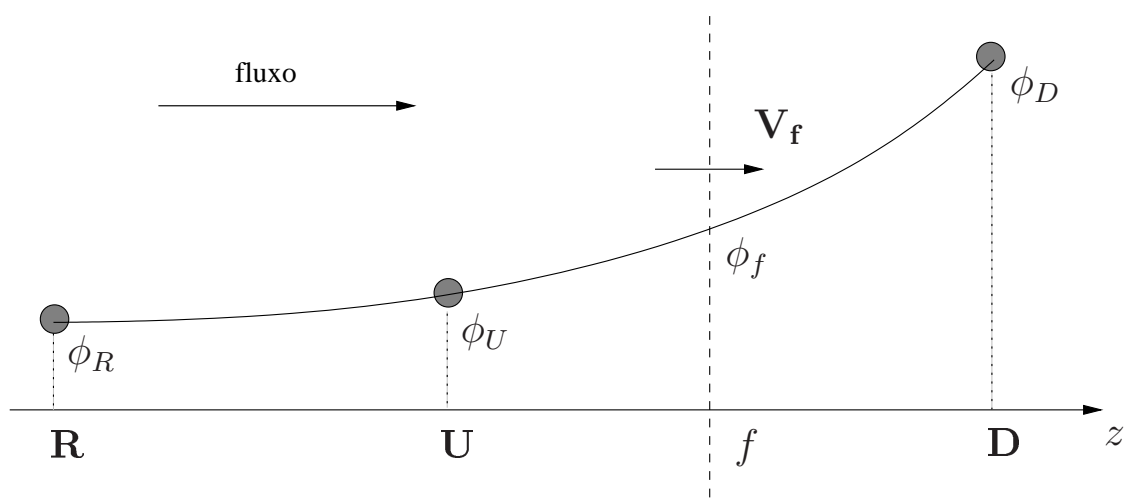

Figura 3.3: Representação esquemática do comportamento monotônico local da variável genérica $\phi$ nas vizinhanças da face $f$

É importante notar que, a partir dessa figura e da definição (3.6), tem-se que $\hat{\phi}_{R}=0$ e $\hat{\phi}_{D}=1$. E, ainda, se $\hat{\phi}_{U}=0$ então $\phi_{U}=\phi_{R}$ e se $\hat{\phi}_{U}=1$ então $\phi_{U}=\phi_{D}$.

\subsection{Critério de limitação CBC}

Num problema de escoamento de fluidos, independentemente da distribuição do campo de velocidade no domínio de solução, as não-linearidades nas equações de transporte não devem violar os valores limite da propriedade transportada $\phi$, dados a partir de uma distribuição conhecida.

Se a variável $\phi$ varia, inicialmente, entre $\alpha_{1}$ e $\alpha_{2}$ então os efeitos devidos aos termos advectivos nunca deverão produzir valores de $\phi$ além desses limites. A importância de soluções limitadas no transporte de propriedades físicas é preservar essa propriedade na forma discre- 
tizada dos termos advectivos. Essencialmente, uma solução é limitada se o seu valor em um ponto computacional é limitado pelos valores da solução nos pontos vizinhos.

Para evitar oscilações na solução numérica exige-se que $\phi_{U}$ e, consequentemente, $\phi_{f}$ sejam localmente limitados entre $\phi_{D}$ e $\phi_{R}$, significando que $\phi_{R} \leqslant \phi_{U} \leqslant \phi_{D}$, ou que $\phi_{R} \geqslant \phi_{U} \geqslant \phi_{D}$. Se esse critério está satisfeito para todo ponto no domínio de solução, a solução global estará ausente de oscilações não-físicas. Esta é a essência do tratamento dos termos advectivos em variáveis normalizadas.

Gaskell e Lau [47] formularam o critério CBC, a qual está ilustrado geometricamente na Figura 3.4. O critério CBC para $\phi_{U}$ é dado por

$$
0 \leqslant \hat{\phi}_{U} \leqslant 1
$$

Esse critério afirma que se a função $\hat{\phi}_{f}$ em (3.7) é crescente e contínua (ou crescente e contínua por partes), então uma aproximação por diferenças finitas para $\hat{\phi}_{f}$ é limitada se, e somente se, satisfaz as seguintes condições (Gaskell e Lau [47])

- $\hat{\phi}_{f}\left(\hat{\phi}_{U}\right) \leqslant 1, \quad \hat{\phi}_{f}\left(\hat{\phi}_{U}\right) \geqslant \hat{\phi}_{U}, \quad \hat{\phi}_{f}(0)=0 \quad$ e $\quad \hat{\phi}_{f}(1)=1, \quad$ se $\quad \hat{\phi}_{U} \in[0,1]$,

- $\hat{\phi}_{f}\left(\hat{\phi}_{U}\right)=\hat{\phi}_{U}, \quad$ se $\quad \hat{\phi}_{U} \notin[0,1]$.

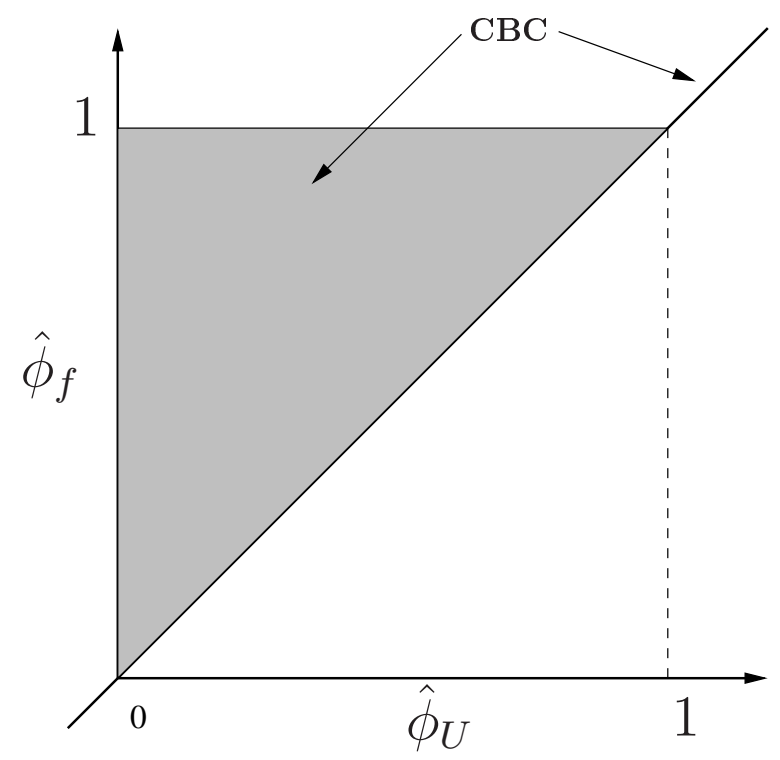

Figura 3.4: Região CBC no plano $\hat{\phi}_{f}-\hat{\phi}_{U}$

A importância do critério CBC (ver Choi et al. [20]) é fornecer uma condição necessária e suficiente para garantir que a solução a ser obtida por um esquema advectivo seja limitada.

Nas últimas décadas muitas tentativas têm sido feitas para derivar o esquema advectivo "upwind" de alta resolução perfeito, isto é, aquele que satisfaz as seguintes propriedades: (i) manter estabilidade da solução numérica; (ii) capturar descontinuidades sem a presença de 
oscilações ou, em casos extremos, com oscilações limitadas; (iii) ser computacionalmente simples e econômico; (iv) conseguir convergência; e (v) ser efetivo em uma variedade de situações. Dentre essas tentativas destacam-se a proposta do esquema HLPA ("Hybrid-Linear Parabolic Approximation") de Zhu [165], do SMART ("Sharp and Monotonic Algorithm for Realistic Transport") por Gaskell e Lau [47], do WACEB por Song et al. [126], do VONOS de Varonos e Bergeles [149] e a proposta do CUBISTA de Alves et al. [2]. Por satisfazerem o critério de estabilidade CBC, tais esquemas vêm sendo utilizados com sucesso numa variedade de aplicações práticas (veja os trabalhos Alhuimaizi [1], Burguete et al. [15], Gross et al. [50], Le Roy et al. [116], Lei et al. [78], Mudryk e Murray [98], Oldenburg e Pruess [104], Ouahsine e Smaoui [105], Silva [122], Trac e Pen [146], Zhao et al. [164], entre muitos outros). Entretanto, a ausência do número de Courant (veja LeVeque [83]) em suas formulações, além da falta de robustez em resolver problemas de evolução, e o desejo de simular problemas de escoamento de fluidos em condições adversas motivaram a derivação do ADBQUICKEST (uma versão limitada do esquema QUICKEST de Leonard [79]).

Vale mencionar que há também outra classe de esquemas de alta resolução sofisticados chamados ENO ("Essentially Non-Oscillatory") e seus relacionados WENO ("Weighted" ENO), que são apropriados para capturar descontinuidades e pontos extremos com alta ordem de precisão (ver Harten et al. [57]). Nesses esquemas, alta ordem é alcançada usando polinômios interpoladores de graus elevados que não garantem resultados não oscilatórios. Seus compromissos são relaxar as condições TVD/CBC e, ao invés de considerar moléculas computacionais fixas, escolher moléculas computacionais variáveis. O preço a ser pago por esse procedimento é, além da dificuldade de implementação, o número muito elevado de operações aritméticas requeridas a cada passo no tempo. A diferença entre ENO/WENO e esquemas TVD/CBC é que os esquemas ENO/WENO têm a propriedade de reter a mesma ordem de precisão espacial em todo o domínio, inclusive nas vizinhanças de descontinuidades e pontos extremos, enquanto que os esquemas TVD/CBC oferecem primeira ordem de precisão nessas regiões críticas. Em outras palavras, a dissipação numérica contida nos esquemas ENO/WENO é intrinsicamente menor que aquela nos esquemas TVD/CBC. Por outro lado, em muitas aplicações - como os escoamentos incompressíveis - segunda ordem de precisão é suficiente (ver Kupferman e Tadmor [71]). Também, esquemas ENO/WENO são usados na simulação da turbulência com LES, em que se conclui frequentemente que estes esquemas são muitos dissipativos.

\subsection{O esquema ADBQUICKEST}

Outra motivação para desenvolvimento do esquema ADBQUICKEST foi os resultados numéricos para a equação (2.1), para $a=1$, obtidos com os esquemas Upwind de Spalding [127], 
Lax-Wendroff de Lax e Wendroff [77] e QUICKEST de Leonard [79] usando a condição inicial

$$
u_{0}(x)= \begin{cases}e^{-\log (50)\left(\frac{x-0.2}{0.15}\right)^{2}}, & 0<x<0.3 \\ 1, & 0.5<x<0.7 \\ 0, & \text { caso contrário. }\end{cases}
$$

As Figuras 3.5, 3.6 e 3.7 mostram as comparações entre as soluções numéricas obtidas com os esquemas Upwind, Lax-Wendroff e QUICKEST, respectivamente, e a solução exata. Para este exemplo linear (simples), fica evidente a baixa qualidade das soluções numéricas obtidas com tais esquemas.

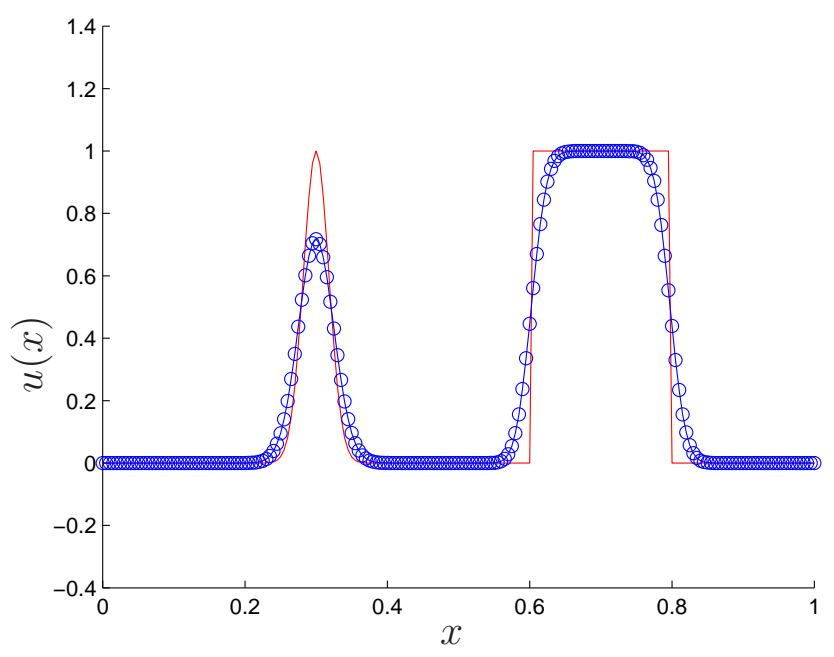

Figura 3.5: Comparação entre a solução numérica obtida com o esquema Upwind (símbolo azul) e a solução exata (linha vermelha), para a equação de transporte de um escalar.

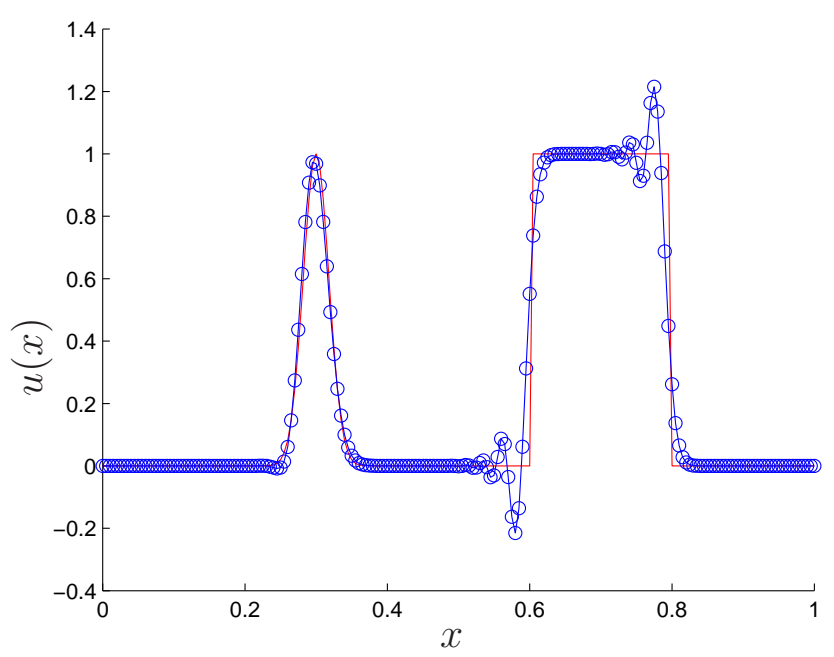

Figura 3.6: Comparação entre a solução numérica obtida com o esquema Lax-Wendroff (símbolo azul) e a solução exata (linha vermelha), para a equação de transporte de um escalar. 


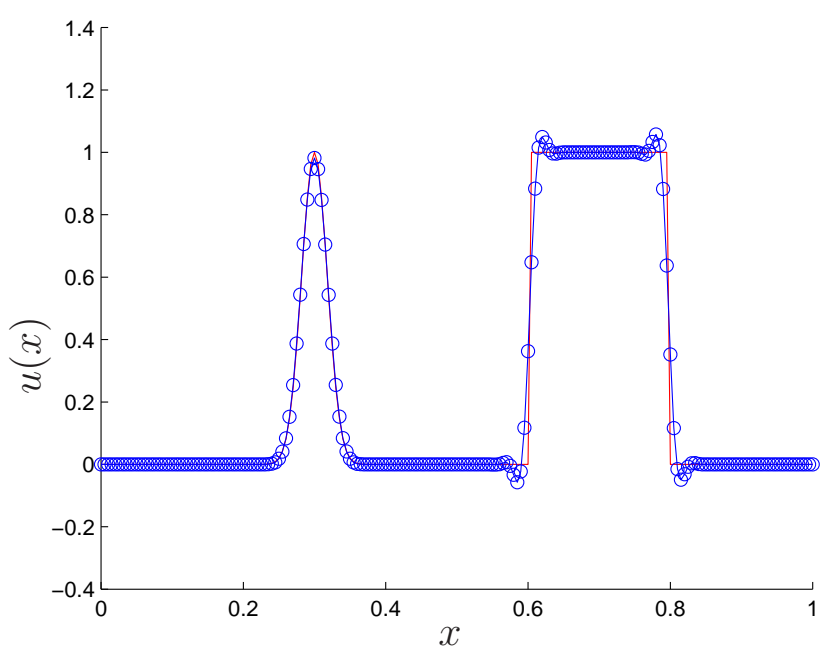

Figura 3.7: Comparação entre a solução numérica obtida com o esquema QUICKEST (símbolo azul) e a solução exata (linha vermelha), para a equação de transporte de um escalar.

O esquema ADBQUICKEST é derivado definindo, por exemplo, o fluxo numérico em $i+1 / 2$ (ver Figura 3.2) em termos de fluxos numéricos conhecidos, isto é,

$$
u_{i+1 / 2}^{n+1}=u_{L_{i+1 / 2}}^{n}+\varphi_{i+1 / 2}^{n}\left(u_{H_{i+1 / 2}}^{n}-u_{L_{i+1 / 2}}^{n}\right),
$$

em que $u_{L_{i+1 / 2}}^{n}$ e $u_{H_{i+1 / 2}}^{n}$ são fluxos numéricos de baixa e alta ordem, respectivamente, e $\varphi_{i+1 / 2}^{n}$ é uma função limitadora do fluxo, uma espécie de "sensor" que detecta regiões suaves e regiões de altos gradientes. A idéia básica do esquema ADBQUICKEST é usar fluxos numéricos de baixa ordem quando necessário e de alta precisão quando possível. Assim, o limitador de fluxo, $\varphi_{i+1 / 2}^{n}$, no tempo $t=t_{n}$, que a partir de agora, omitindo por simplicidade a dependência temporal, deve ser calculado de maneira que em regiões suaves ele valha 1 e 0 em regiões de gradientes elevados ou descontinuidades.

Objetivando minimizar a difusão numérica evidenciada pelo uso dos esquemas Upwind, Lax-Wendroff e QUICKEST, como pode ser visto nas Figuras 3.5, 3.6 e 3.7, propõe-se, em analogia à proposta de Sweby (ver Sweby [131]), o fluxo numérico

$$
u_{f}=u_{i+1 / 2}=u_{U}+\varphi\left(r_{i+1 / 2}\right) \frac{1}{2}(1-|\theta|)\left(u_{D}-u_{U}\right),
$$

em que $r_{i+1 / 2}=r_{f}$ é um parâmetro de suavização, definido como a razão de dois gradientes consectivos, isto é,

$$
r_{f}=\frac{\left(\frac{\partial u}{\partial x}\right)_{g}}{\left(\frac{\partial u}{\partial x}\right)_{f}}
$$


o qual para malhas uniformes reduz-se a

$$
r_{f}=\frac{u_{U}-u_{R}}{u_{D}-u_{U}}
$$

$\mathrm{Ou}$

$$
r_{f}= \begin{cases}\frac{u_{i}-u_{i-1}}{u_{i+1}-u_{i}}, & \text { se } a>0, \\ \frac{u_{i+1}-u_{i+2}}{u_{i}-u_{i+1}}, & \text { se } a<0 .\end{cases}
$$

O parâmetro $r_{i+1 / 2}$ é usado para medir suavidade na solução, pois quando a solução não varia muito, ele se aproxima de 1 , e quando a solução varia de maneira significativa, $r_{i+1 / 2}$ é diferente de 1. E com escolhas diferentes de $\varphi$, conseguem-se esquemas diferentes. Por exemplo, se $\varphi \equiv 0$ obtém-se o esquema Upwind de primeira ordem e quando $\varphi \equiv 1$ e $a>0$ deriva-se o esquema de Lax-Wendroff.

A estratégia para a derivação do esquema ADBQUICKEST é escolher $\varphi$ de maneira que o esquema resultante seja TVD e de terceira ordem de precisão em regiões suaves, ou seja, adota-se o esquema QUICKEST de Leonard para aproximar a solução em regiões suaves.

Da aplicação do critério de Harten [56] no esquema (3.4) (em que $\phi=u$ ) com função fluxo numérico dada por (3.11), obtém-se que o esquema é TVD, sob a condição CFL $|\theta| \leq 1$, se o limitador de fluxo $\varphi$ satisfaz as restrições TVD de Sweby [131] (ou princípio de monotonicidade)

$$
\begin{cases}\varphi(r)=0, & \text { para } r \leq 0 \\ 0 \leq \varphi(r) \leq \min \{2,2 r\}, & \text { para } r>0\end{cases}
$$

As condições (3.15) significam que o gráfico do limitador no plano $(\varphi, r)$ deve estar contido na região hachurada da Figura 3.8.

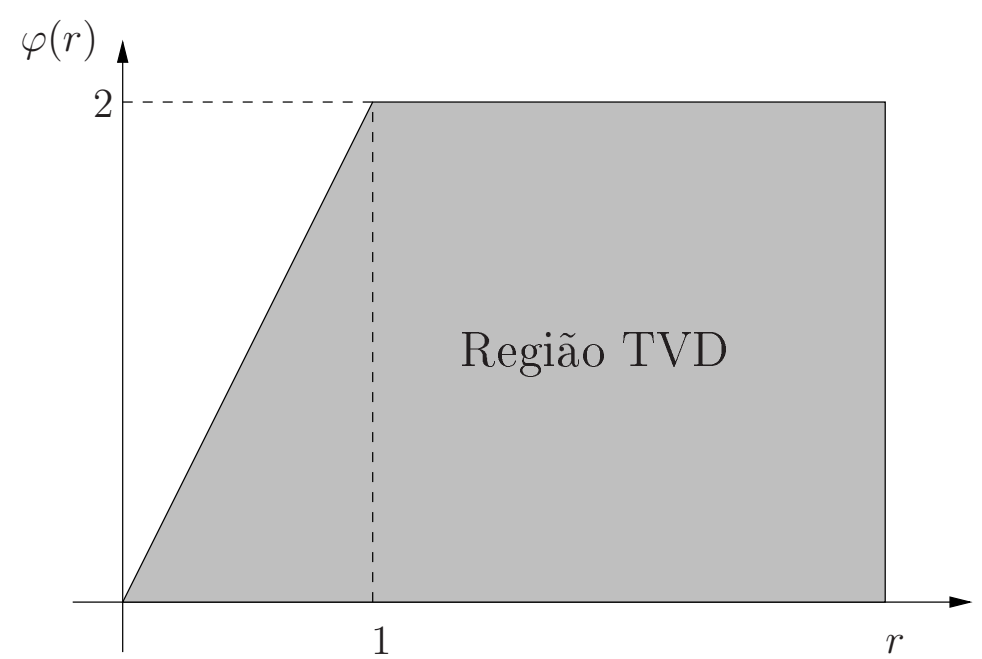

Figura 3.8: Região TVD de Sweby. 
É bem conhecido (ver Sweby [131]) que se $\varphi$ satisfaz $\varphi(1)=1$ e admite derivadas laterais (à esquerda e à direita) no ponto $r=1$, então, longe de pontos extremos, o esquema dado por (3.4) e (3.11) é de segunda ordem de precisão no domínio de regularidade da solução; e para alcançar terceira ordem a sua inclinação no ponto $(r, \varphi(r))=(1,1)$ deve ser $(1+\theta) / 3$ (ver Arora e Roe [7]). Essas são as condições adotadas no desenvolvimento do limitador $\varphi$ para o esquema ADBQUICKEST.

Em variáveis normalizadas de Leonard, o esquema (3.4) com fluxo numérico dado por (3.11) pode ser escrito como

$$
\begin{aligned}
\hat{u}_{U}^{n+1} & =\hat{u}_{U}-\theta\left(\hat{u}_{f}-\hat{u}_{g}\right), \\
\hat{u}_{f} & =\hat{u}_{U}+\varphi_{f} \frac{1}{2}(1-|\theta|)\left(1-\hat{u}_{U}\right),
\end{aligned}
$$

em que $\varphi_{f}=\varphi\left(r_{f}\right)$ com

$$
r_{f}=\frac{u_{U}-u_{R}}{u_{D}-u_{U}}=\frac{\hat{u}_{U}}{1-\hat{u}_{U}} .
$$

A partir das restrições (3.15), a relação funcional em (3.17) envolvendo $\hat{u}_{f}$ e $\hat{u}_{U}$ que satisfaz o princípio TVD pode ser reescrita como

$$
\begin{cases}\hat{u}_{U} \leq \hat{u}_{f} \leq \min \left\{1-|\theta|+|\theta| \hat{\phi}_{U},(2-|\theta|) \hat{u}_{U}\right\}, & \text { para } 0<\hat{\phi}_{U}<1 \\ \hat{u}_{f}=\hat{u}_{U}, & \text { para } \quad \hat{u}_{U} \leq 0 \quad \text { ou } \quad \hat{u}_{U} \geq 1 .\end{cases}
$$

Esta região está mostrada na Figura 3.9 (parte verde) quando $a>0$ a Courant $\theta=0.5$.

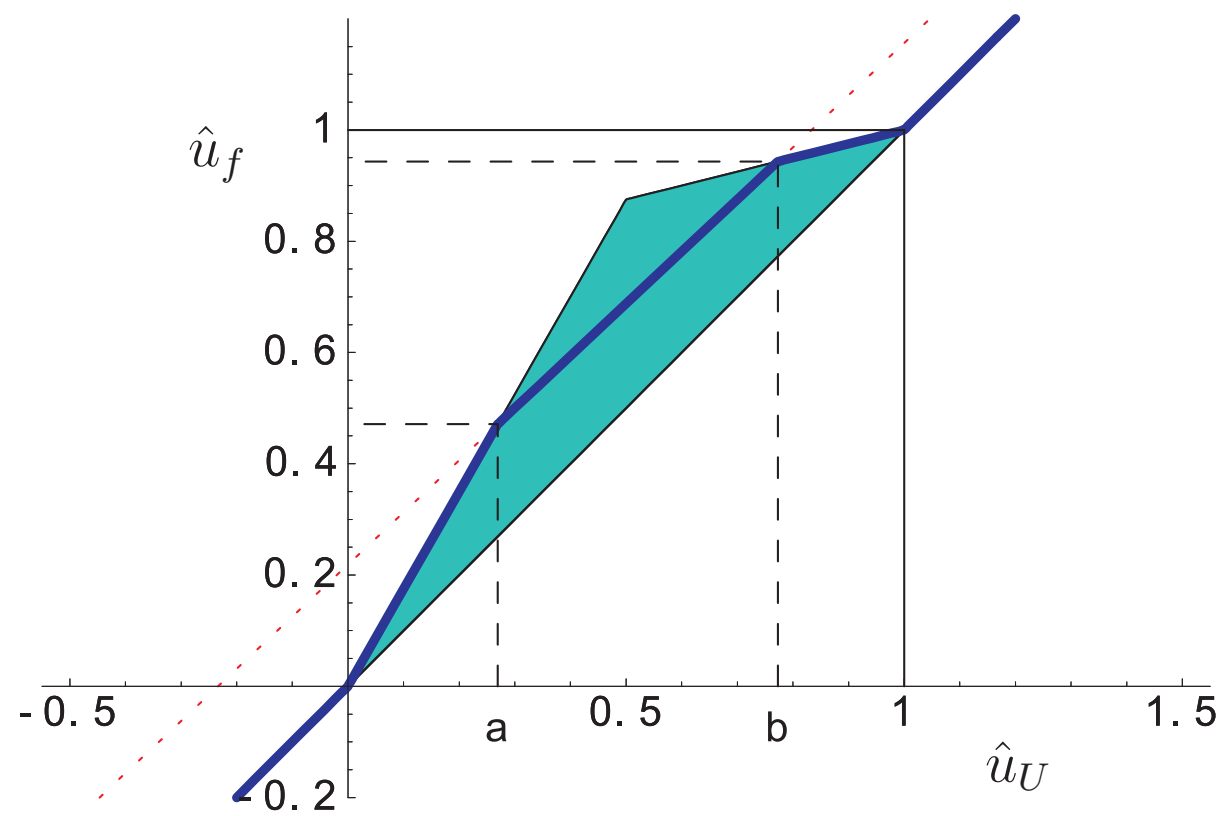

Figura 3.9: Gráfico da variável normalizada de $\hat{u}_{f}$ para o esquema QUICKEST (vermelho) e o esquema ADBQUICKEST $\operatorname{com} \theta=0.5$ (azul). 
Das equações (3.17) e (3.18), segue que a relação correspondente a $\varphi(1)=1$ (segunda ordem de precisão) em variáveis normalizadas é

$$
\hat{u}_{f}\left(\frac{1}{2}\right)=\frac{3}{4}-\frac{1}{4}|\theta| \text {. }
$$

Para terceira ordem, segue da equação (3.17) a condição equivalente

$$
\left.\frac{d \hat{u}_{f}}{d \hat{u}_{U}}\right|_{\frac{1}{2}}=1+\frac{1}{2}(1-|\theta|)\left(\frac{2 \theta-1}{3}\right) .
$$

O esquema ADBQUICKEST é obtido impondo-se que o fluxo numérico na interface (normalizado) seja monotônico e satisfaz as equações (3.19), (3.20) e (3.21). Em outras palavras, o esquema ADBQUICKEST leva o esquema QUICKEST de Leonard em sua formulação, por este último satisfazer às relações (3.20) e (3.21), e adota idéias similares àquelas de Alves et al. [2], Leonard [81] e Song et al. [126]. Em suma, o ADBQUICKEST - um melhoramento no esquema QUICKEST de Leonard [79] - é definido por

$$
\hat{u}_{f}= \begin{cases}(2-|\theta|) \hat{u}_{U}, & 0<\hat{u}_{U}<a, \\ \hat{u}_{U}+\frac{1}{2}(1-|\theta|)\left(1-\hat{u}_{U}\right)-\frac{1}{6}\left(1-\theta^{2}\right)\left(1-2 \hat{u}_{U}\right), & a \leq \hat{u}_{U} \leq b, \\ 1-|\theta|+|\theta| \hat{u}_{U}, & b<\hat{u}_{U}<1, \\ \hat{u}_{U}, & \hat{u}_{U} \notin[0,1],\end{cases}
$$

em que $a$ e $b$ são, respectivamente, os pontos de intersecção entre as curvas $\hat{u}_{f}$ do QUICKEST e $\hat{u}_{f}=(2-|\theta|) \hat{u}_{U}$, e $\hat{u}_{f}$ do QUICKEST e $\hat{u}_{f}=1-|\theta|+|\theta| \hat{u}_{U}$, isto é,

$$
a=\frac{2-3|\theta|+\theta^{2}}{7-9|\theta|+2 \theta^{2}} \quad \text { e } \quad b=\frac{-4+3|\theta|+\theta^{2}}{-5+3|\theta|+2 \theta^{2}} .
$$

A Figura 3.9 mostra ambos os esquemas QUICKEST e ADBQUICKEST (QUICKEST adaptativo) no plano $\left(\hat{u}_{f}, \hat{u}_{U}\right)$.

Utilizando-se a definição (3.8), o esquema (3.22) em variáveis não-normalizadas pode ser representado como

$$
u_{f}=\left\{\begin{array}{lll}
(2-|\theta|) u_{U}-(1-|\theta|) u_{R}, & \text { se } & 0<\hat{u}_{U}<a, \\
\alpha_{D} u_{D}+\alpha_{U} u_{U}-\alpha_{R} u_{R}, & \text { se } \quad a \leq \hat{u}_{U} \leq b, \\
(1-|\theta|) u_{D}+|\theta| u_{U}, & \text { se } \quad b<\hat{u}_{U}<1, \\
u_{U}, & \text { se } \quad \hat{u}_{U} \notin[0,1],
\end{array}\right.
$$


no qual

$$
\begin{aligned}
\alpha_{D} & =\frac{1}{6}\left(2-3|\theta|+\theta^{2}\right) \\
\alpha_{U} & =\frac{1}{6}\left(5+3|\theta|-2 \theta^{2}\right) \\
\alpha_{R} & =\frac{1}{6}\left(1-\theta^{2}\right) .
\end{aligned}
$$

O limitador de fluxo correspondente para o esquema ADBQUICKEST é obtido das equações (3.17), (3.18) e (3.22), cujo resultado é

$$
\varphi\left(r_{f}\right)= \begin{cases}2 r_{f}, & 0<r_{f}<p \\ \frac{2+\theta^{2}-3|\theta|+\left(1-\theta^{2}\right) r_{f}}{3-3|\theta|}, & p \leq r_{f} \leq q \\ 2, & r_{f}>q \\ 0, & r_{f}<0,\end{cases}
$$

em que

$$
p=\frac{-2-\theta^{2}+3|\theta|}{-5-\theta^{2}+6|\theta|} \quad \text { e } \quad q=\frac{-4+\theta^{2}+3|\theta|}{\theta^{2}-1} .
$$

Das equações (3.22) e (3.23), pode-se dizer que o esquema ADBQUICKEST é adaptativo no sentido de que a aproximação para o fluxo numérico é definida dependendo da suavidade da solução.

O limitador em (3.23) pode ser escrito também na forma mais utilizada na literatura (veja, por exemplo, Waterson e Deconinck [154]) como

$$
\varphi\left(r_{f}\right)=\max \left\{0, \min \left[2 r_{f}, \frac{2+\theta^{2}-3|\theta|+\left(1-\theta^{2}\right) r_{f}}{3-3|\theta|}, 2\right]\right\} .
$$

Dentro da região TVD de Sweby, o gráfico do limitador de fluxo do ADBQUICKEST para vários valores de $\theta$ é apresentado na Figura 3.10, onde pode-se notar que, para todos os valores do parâmetro $\theta$, o limitador de fluxo passa pelo ponto $(1,1)$ na região TVD a qual é uma condição necessária e suficiente para segunda ordem de precisão longe de extremos (ver Sweby [131]), e corresponde ao ponto $(0.5,0.75-0.25|\theta|)$ no diagrama de variáveis normalizadas de Leonard [80] (veja a região CBC em Gaskell e Lau [47] ou Waterson e Deconinck [154]). Pode-se observar também que, quando o parâmetro $\theta$ tende a 0 , o fluxo numérico (3.22) passa pelo ponto $(0.5,0.75)$ no diagrama de variáveis normalizadas a qual é uma condição necessária e suficiente para segunda ordem e corresponde ao ponto $(1,1)$ na região TVD de Sweby. 


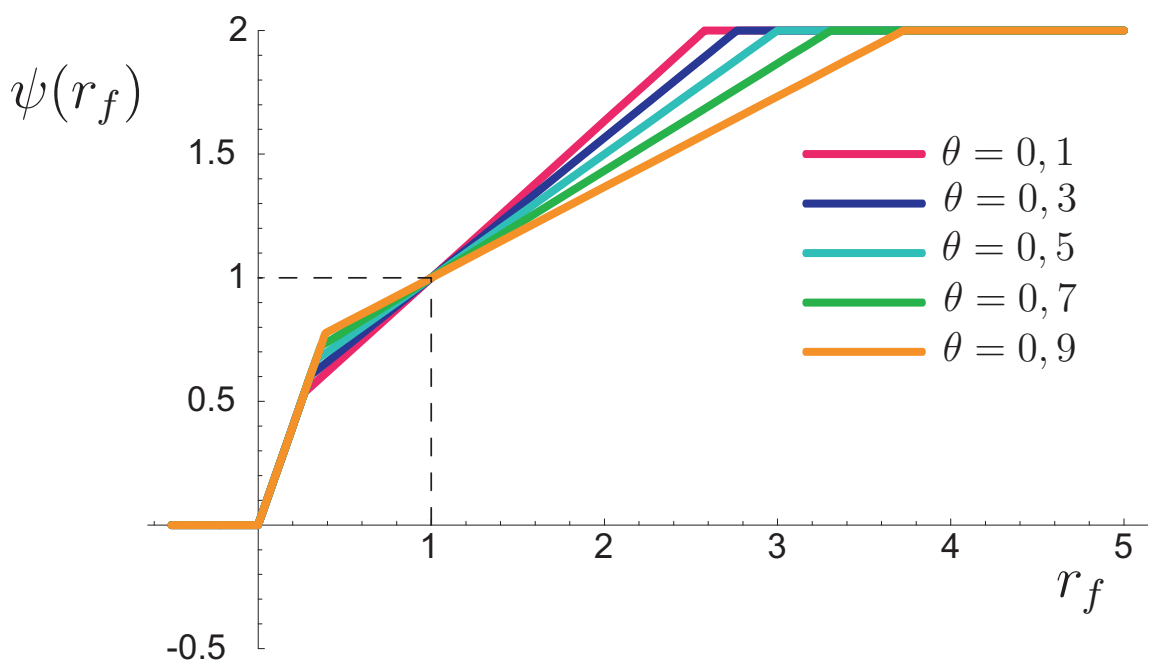

Figura 3.10: Limitador de fluxo do esquema ADBQUICKEST na região TVD de Sweby para vários valores do número de Courant $\theta$. 


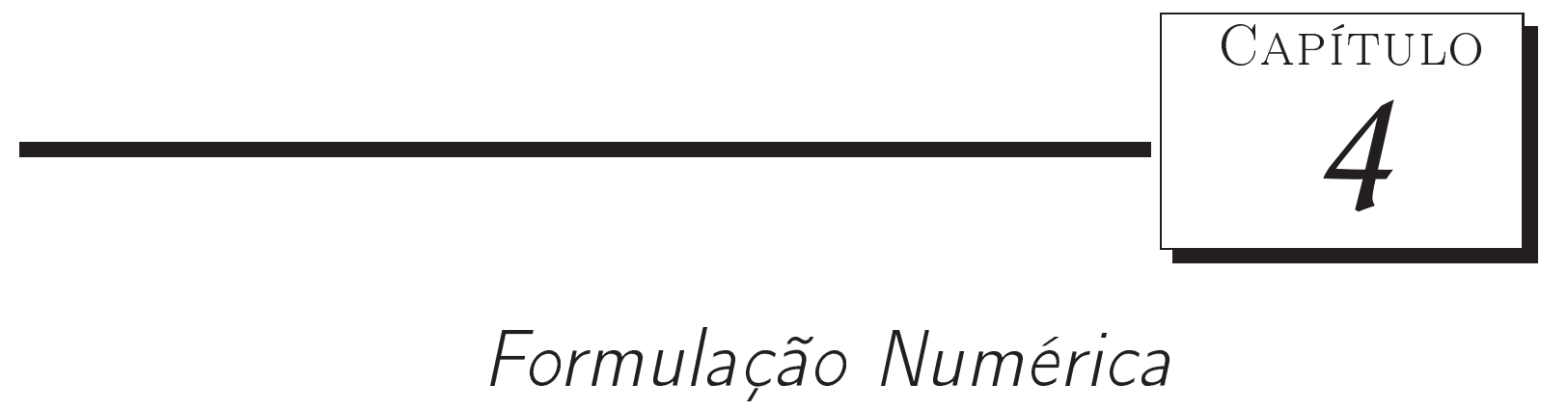

Neste capítulo, é apresentada uma breve descrição do método MAC para a solução numérica de escoamentos incompressíveis transientes com superfícies livres móveis. São discutidos, também, o método numérico (projeção de Chorin [21]), o procedimento computacional de solução das equações discretas, e a discretização das equações pelo método das diferenças finitas.

\subsection{A metodologia MAC}

O método MAC ("Marker-and-Cell"), desenvolvido por Harlow e Welch [53], foi uma dos primeiros passos bem sucedidos a ser utilizado em escoamentos incompressíveis com superfícies livres. Ela emprega uma malha euleriana, em que a posição do fluido no interior da malha é determinado por um conjunto de partículas marcadoras que movem-se com o fluido. As localizações dessas partículas são obtidas de acordo com o campo de velocidade computado. Detalhes sobre as vantagens e limitações do método MAC estão apresentados no trabalho de Nichols e Hirt [99].

Vários pesquisadores apresentaram variações, modificações e generalizações da metodologia MAC. Dentre alguns deles, podem-se citar os trabalhos de Hirt e Shannon [61] os quais aplicaram as condições de contorno corretas na fronteira livre do fluido. Hirt e Cook [60] desenvolveram uma variante do método MAC para descrever escoamentos transientes tridimensionais com geometria irregulares. Amsden e Harlow [4] introduziram o método SMAC ("Simplified Marker-and-Cell") para eliminar as dificuldades associadas com a aplicação das condições de fronteira do método MAC. Baseado na metodologia SMAC, Tomé e McKee [143] desenvolveram o método GENSMAC ("Generalized-Simplified-Marker-and-Cell") para a simulação de escoamentos newtonianos com superfícies livres em domínios 2D arbitrários. Visando resolver problemas 3D com superfícies livres, Tomé et al. [140] desenvolveram a metodologia 
GENSMAC3D, que é uma extensão do método GENSMAC. Atualmente a metodologia MAC continua em uso por vários pesquisadores (ver, por exemplo, os trabalhos atuais de Oishi [102], Oishi et al. [103] e McKee et al. [95]).

Neste trabalho, as partículas marcadoras são baseadas na metodologia GENSMAC, ou seja, as partículas marcadoras são mantidas apenas nas superfícies e não em todo domínio como no método MAC. Esse método visa economizar tempo de processamento e memória nas simulações numéricas.

\subsubsection{Malha computacional}

Existem várias maneiras de se distribuir as variáveis $u, v, w, p_{e}, \kappa \mathrm{e} \varepsilon$ (caso tridimensional) quando não se exige que elas estejam armazenadas na mesma posição. Uma delas é utilizando a malha deslocada ("staggered grid"), na qual as variáveis estão armazenadas em posições diferentes. Esse tipo de malha foi introduzida inicialmente por Harlow e Welch [53] e se tornou padrão no cálculo de escoamentos incompressíveis.

A malha deslocada é muito utilizada no contexto da metodologia MAC por apresentar algumas propriedades interessantes, tais como: é computacionalmente simples, garantem localmente a conservação de massa, quantidade de movimento, energia cinética e vorticidade (Perot e Nallapati [108]); e, é favorável para o uso de métodos de projeção no cálculo da pressão.

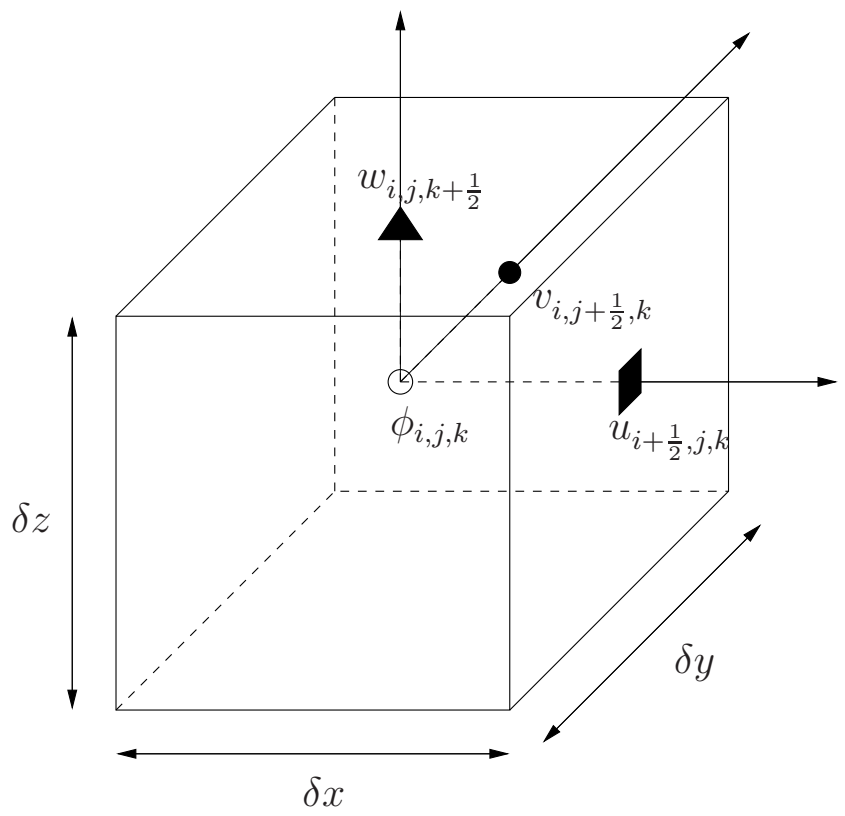

Figura 4.1: Célula computacional típica que mostra onde as variáveis são discretizadas. A variável genérica $\phi$ representa $p_{e}, \kappa, \varepsilon$ ou $\nu_{t}$.

Nesse tipo de malha, para escoamentos 3D, as velocidades são definidas nas faces da célula, enquanto que a pressão no centro da célula, como está ilustrado na Figura 4.1. 


\subsubsection{Classificação das células}

Por se tratar de escoamentos em que o fluido está continuamente em movimento, torna-se necessário fazer uma classificação das células da malha para que se identifique a região do fluido. Essa classificação permite identificar se a célula contém ou não fluido, se faz parte da entrada ou saída, se é uma célula do contorno rígido ou estão na superfície livre. Dessa forma, as propriedades do escoamento são representadas em uma malha de células retangulares. Para tanto, adota-se a seguinte classificação de células:

- Células vazias - "Empty" (E): São células que não contêm fluido;

- Células cheias - "Full" (F): São células cheias de fluido;

- Células de superfície livre - "Surface" (S): São células que contêm fluido e que possuem pelo menos uma face em contato com célula vazia;

- Células de contorno - "Boundary" (B): São células que definem um contorno rígido de maneira que as condições de contorno possam ser impostas;

- Células do injetor - "Inflow" (I): São células que definem a entrada de fluido no domínio;

- Células do ejetor - "Outflow" (O): São células que definem a saída de fluido no domínio.

A Figura 4.2 ilustra um exemplo de domínio e a estrutura de células na malha em um dado instante.

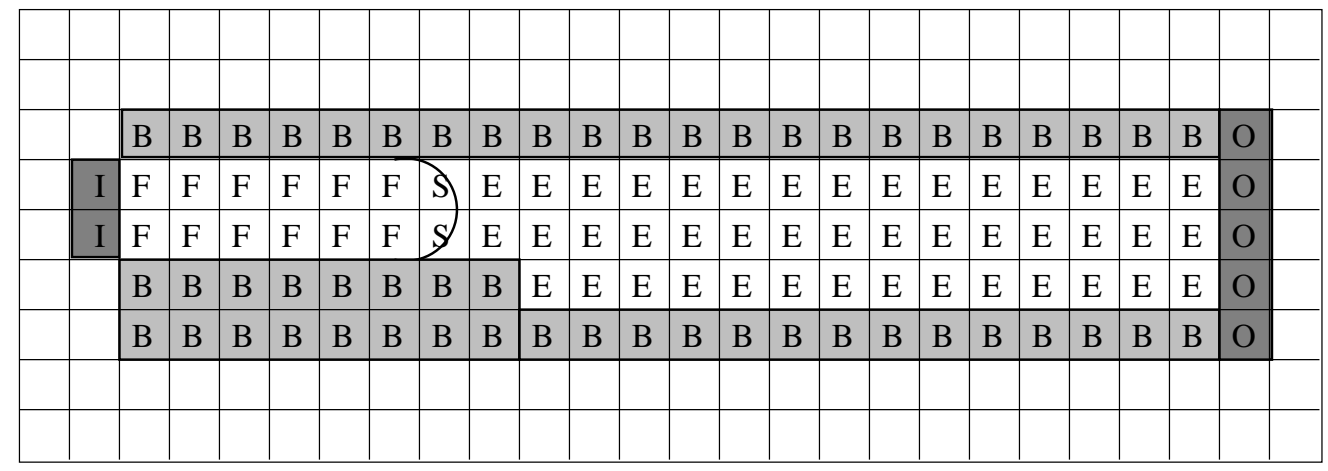

Figura 4.2: Tipos de células no domínio computacional. 


\subsection{Método de projeção}

Na literatura existem muitos métodos que resolvem problemas de escoamentos viscosos, incompressíveis, newtonianos transientes com superfícies livres modelados pelas equações (2.42) e (2.43). Essas equações são resolvidas utilizando-se a formulação de variáveis primitivas (Dennis et al. [29]) de forma acoplada ou desacoplada.

Os métodos que resolvem simultaneamente o sistema de equações (2.42) e (2.43) em cada passo do tempo, são chamados de métodos de solução acoplados, e os métodos que resolvem as equações separadamente são chamados de métodos segregados.

Os métodos de solução acoplados são muitas vezes descartados em escoamentos realísticos pois apresentam dificuldades na sua implementação e um custo computacional alto. Essa ineficiência é devido a dificuldade na solução do sistema (2.42) e (2.43) que é extenso e por receber fortes influências das não-linearidades dos termos advectivos. Desta forma, desacoplar as equações é uma metodologia muito utilizada no contexto de resolução das equações de conservação.

Os métodos que utilizam a estratégia do desacoplamento dos campos de pressão e velocidade das equações (2.42) e (2.43) foram propostos originalmente por Chorin [21] e Temam [136] e conhecidos como métodos de passo fracionário ou métodos de projeção (ver mais detalhes em Oishi [102]). A vantagem da classe de métodos tipo projeção é que os cálculos dos campos de velocidade e pressão estão desacoplados, ou seja, assume-se que os termos não lineares são tratados explicitamente e resolve-se a cada passo no tempo um conjunto de equações para a velocidade e uma equação escalar do tipo Poisson para a pressão. Esta metodologia utilizada pelos métodos de projeção é computacionalmente eficiente quando comparados com os métodos de solução do tipo acoplado. Um método de solução que utiliza uma variante do método de projeção é a metodologia GENSMAC.

Neste trabalho, adota-se o método projeção na forma desacoplada. A seguir é apresentada uma adaptação desse procedimento de solução nos códigos GENSMAC e GENSMAC3D para a simulação de escoamentos laminares e turbulentos 3D na presença de superfícies livres.

\subsubsection{Procedimento de solução}

Inicialmente, considera-se a discretização temporal das equações (2.42) e (2.43) dada por

$$
\begin{gathered}
\nabla \cdot \mathbf{u}^{(n+1)}=0 \\
\mathbf{u}^{n+1}=\mathbf{u}^{n}+\delta t\left\{-\nabla \cdot(\mathbf{u} \mathbf{u})^{n}-\nabla p_{e}^{n+1}+R e^{-1} \nabla^{2} \mathbf{u}^{n}+\left(F r^{2}\right)^{-1} \mathbf{g}^{n}+R e^{-1} \nabla \cdot\left(\nu_{t} \mathbf{D}\right)^{n}\right\}
\end{gathered}
$$

A idéia básica dos métodos de projeção é utilizar a equação da quantidade de movimento (4.2) discretizada no tempo e resolver para um campo de velocidade intermediário. Assim, a equação 
(4.2) é reescrita como

$$
\tilde{\mathbf{u}}=\mathbf{u}+\delta t\left\{-\nabla \cdot(\mathbf{u u})-\nabla \tilde{p}_{e}+R e^{-1} \nabla^{2} \mathbf{u}+\left(F r^{2}\right)^{-1} \mathbf{g}+\alpha R e^{-1} \nabla \cdot\left(\nu_{t} \mathbf{D}\right)\right\}^{n},
$$

em que $\tilde{p}_{e}^{n}$ é pressão tentativa, ou seja, uma aproximação para a pressão $p_{e}^{n+1}$. Na equação (4.3) foi introduzido o parâmetro $\alpha$ (constante) com o objetivo de "ligar e desligar a turbulência", isto é, no regime laminar $\alpha=0$ e no turbulento $\alpha=1$. Na maioria das vezes, a velocidade $\tilde{\mathbf{u}}$ não é senoidal, isto é, não satisfaz a equação da continuidade (2.42) pois $p_{e} \neq \tilde{p}_{e}$. Em escoamentos incompressíveis, a função da pressão é fazer com que o campo de velocidade satisfaça a equação da continuidade (2.42). Dessa forma, faz-se necessário o cálculo da pressão num próximo passo.

As condições de contorno em paredes rígidas para ũ na equação (4.3) são impostas como

$$
\begin{aligned}
\tilde{\mathbf{u}} \cdot \mathbf{n} & =\mathbf{u}^{n+1} \cdot \mathbf{n}, \\
\tilde{\mathbf{u}} \cdot \mathbf{m}_{\mathbf{1}} & =\mathbf{u}^{n+1} \cdot \mathbf{m}_{\mathbf{1}}, \\
\tilde{\mathbf{u}} \cdot \mathbf{m}_{\mathbf{2}} & =\mathbf{u}^{n+1} \cdot \mathbf{m}_{\mathbf{2}},
\end{aligned}
$$

ou seja, as condições de contorno impostas para ũ são as mesmas para $\mathbf{u}^{n+1}$ como em (2.80) para problemas de contorno sem deslizamento e, (2.81) para contorno sem aderência. Impõem-se ainda, as mesmas condições de contorno de $\mathbf{u}^{n+1}$, do tipo entrada e saída, para $\tilde{\mathbf{u}}$.

Pelo teorema de Helmholtz-Hodge (ver Hodge [62], Ladyzhenskaja [73], Denaro [28]), o campo de velocidade pode ser decomposto da seguinte forma

$$
\tilde{\mathbf{u}}=\mathbf{u}^{n+1}+\nabla \psi^{n+1}
$$

Aplicando o divergente na equação (4.5) e impondo (4.1) para $\mathbf{u}^{n+1}$ obtém-se uma equação de Poisson para uma pressão tentativa $\psi^{n+1}$ dada por

$$
\nabla^{2} \psi^{n+1}=\nabla \cdot \tilde{\mathbf{u}}
$$

A importância dessa equação elíptica para pressão é que ela faz a ligação entre as equações de quantidade de movimento e continuidade.

As condições de contorno impostas para $\psi$ na equação (4.6) são condições homogêneas tipo Neumann na entrada e contorno rígido e condições homogêneas tipo Dirichlet na superficíe livre e saída.

Substituindo-se (4.5) em (4.3), obtém-se

$$
\frac{\mathbf{u}^{n+1}+\nabla \psi^{n+1}}{\delta t}=\left\{\frac{\mathbf{u}}{\delta t}-\nabla \cdot(\mathbf{u u})-\nabla \tilde{p}_{e}+R e^{-1} \nabla^{2} \mathbf{u}+\left(F r^{2}\right)^{-1} \mathbf{g}+\alpha R e^{-1} \nabla \cdot\left(\nu_{t} \mathbf{D}\right)\right\}^{n} .
$$


Comparando (4.7) com (4.2) tém-se a pressão efetiva atual, isto é,

$$
p_{e}^{n+1}=p_{e}^{n}+\frac{\psi^{n+1}}{\delta t}
$$

em que $\delta t$ é a marcha no tempo.

\subsubsection{Algoritmo computacional}

Para simular escoamentos de fluidos sem a modelagem $\kappa-\varepsilon$, admite-se que, num dado instante de tempo $t_{n}$, as variáveis dependentes são conhecidas e as condições de contorno associadas estão especificadas. As equações de Navier-Stokes instantâneas são resolvidas de maneira segregada. Mais especificamente, um ciclo computacional consiste em atualizar as variáveis discretas, a partir do tempo inicial $t_{0}$ (ou um tempo anterior $t_{n}$ ) no tempo $t_{n+1}=$ $t_{n}+\delta t$, utilizando-se uma série de passos interrelacionados. A sequência desses passos é descrita a seguir.

- Passo 1: Seja $p^{n}=\tilde{p}$ um campo de pressão que satisfaça as condições de contorno na superfície livre. Esse campo de pressão é calculado pela equação (2.97) e as velocidades nas superfícies livres por (2.98) e (2.99).

- Passo 2: Conhecido o campo de pressão, calcular um campo de velocidade intermediário $\tilde{\mathbf{u}}$ em $t_{n+1}$, satisfazendo a equação (4.3) $\operatorname{com} \alpha=0$.

- Passo 3: Resolver a equação de Poisson (4.6) para o potencial $\psi$ utilizando-se a condição homogênea de Dirichlet na superfície livre e saída $(\psi=0)$, e condição homogênea de Neumann em contornos rígidos e entrada $\left(\frac{\partial \psi}{\partial n}=0\right)$.

- Passo 4: Atualizar o campo de velocidade pela equação (4.5).

- Passo 5: Atualizar o campo de pressão a partir de (4.8).

- Passo 6: Determinar as novas posições das partículas marcadoras que representam o fluido. Essas partículas permitem a visualização do escoamento e a orientação da superfície livre. As posições são atualizadas resolvendo

$$
\dot{\mathbf{x}}=\mathbf{u}^{(n+1)}
$$

pelo método de Euler explícito.

- Passo 7: Atualizar as condições de contorno necessárias para o próximo ciclo.

Desta forma, os passos acima formam um ciclo computacional, a partir de um tempo inicial $t_{n}$, para o cálculo das variáveis primitivas $u, v$ e $w$ num tempo posterior $t_{n+1}=t_{n}+\delta t$. 
Para simular os efeitos da turbulência usando a modelagem $\kappa-\varepsilon$, assume-se $\alpha=1$ na equação (4.3), no Passo 1 calcula-se o campo de pressão pela equação (2.101) e as velocidades nas superfícies livres por (2.102) e (2.103) e acrescentam-se os seguintes passos entre os Passo 5 e Passo 6 do algoritmo computacional descrito anteriormente:

- Calcular a energia cinética turbulenta $\kappa$ a partir da discretização de (2.44).

- Calcular a dissipação de energia $\varepsilon$ a partir da discretização de (2.45).

- Atualizar a viscosidade turbulenta $\nu_{t}$ a partir de (2.47).

Como feito anteriormente, calculam-se as variáveis primitivas $u, v, w, \kappa, \varepsilon$ e $\nu_{t}$ no tempo posterior $t_{n+1}$.

\subsubsection{Restrição no valor do passo temporal}

As consequências da escolha do passo temporal são cruciais para a simulação dos regimes laminar e turbulento, e o seu valor deve ser estimado de maneira que os erros de arredondamento tenham um crescimento controlado.

Durante a execução do ciclo computacional descrito na seção anterior (tanto para o caso $\alpha=0$ como para $\alpha=1$ ), as restrições no passo temporal são as mesmas discutidas em Tomé et al. [140]. Mais especificamente, quando o método Euler explícito é utilizado para a marcha no tempo na equação de quantidade de movimento, uma condição necessária é a restrição de estabilidade parabólica. Essa restrição, no caso 3D, é dada pela seguinte expressão

$$
\delta t_{v i s c} \leq \frac{R e}{2}\left(\frac{1}{\delta x^{2}}+\frac{1}{\delta y^{2}}+\frac{1}{\delta z^{2}}\right)^{-1}
$$

em que $\delta t_{v i s c}$ é o passo temporal adimensional resultante da condição de estabilidade nos termos viscosos, e $\delta x, \delta y$ e $\delta z$ são os espaçamentos adimensionais da malha nas direções $x, y$ e $z$ respectivamente.

Do fato de que uma partícula de fluido não pode percorrer, em cada passo de tempo, uma distância maior do que o incremento espacial, as seguintes restrições são derivadas

$$
\delta t_{c f l} \leq \min \left\{\delta t_{u}, \delta t_{v}, \delta t_{w}\right\}
$$

na qual

$$
\delta t_{u} \leq\left(\frac{\delta x}{|u|_{\max }}\right), \quad \delta t_{v} \leq\left(\frac{\delta y}{|v|_{\max }}\right) \quad \text { e } \quad \delta t_{w} \leq\left(\frac{\delta x}{|w|_{\max }}\right)
$$

em que $|u|_{\max },|v|_{\max }$ e $|w|_{\max }$ são, respectivamente, os valores máximos em modulo das velocidades $\tilde{u}, \tilde{v}$ e $\tilde{w}$ em cada inicio do ciclo computacional. Essas restrições estão relacionadas à condição Courant-Friedrichs-Lewy (CFL) (Courant et al. [22]). 
O valor de $\delta t$ escolhido deve satisfazer, simultaneamente, as condições (4.10) e (4.11), ou seja

$$
\delta t=\tau \min \left\{\delta t_{v i s c}, \delta t_{c f l}\right\}
$$

na qual $0<\tau \leq 1$.

Vale ressaltar que esses critérios representam apenas condições necessárias para a estabilidade das equações, mas não, obrigatoriamente, suficiente.

\subsection{Discretização das Equações}

Nesta seção são apresentadas as discretizaçãoes das equações de conservação e condições de contorno necessárias para resolver numericamente as equações apresentada na subseção 4.2.1.

As derivadas temporais são aproximadas por diferenças avançadas (Euler explícito), enquanto que, os termos difusivos e os gradientes de pressão são discretizados por diferenças centrais de segunda ordem. Os termos advectivos nos modelos são discretizados utilizando-se o esquema "upwind" de alta resolução ADBQUICKEST, discutidos na seção 3.5. A equação de Poisson, para o potencial $\psi$, é aproximada pelo operador laplaciano de sete pontos (caso 3D), em que o sistema de equações lineares correspondente é resolvido pelo método dos gradientes conjugados.

Para resolver numericamente as equações (2.63)-(2.68), o sistema de malha deslocada de Harlow e Welch [53] é adotado, como mostra a Figura 4.1. As equações são discretizadas localmente em cada célula computacional. Nesse tipo de malha, as pressões $\tilde{p}, \tilde{p}_{e}$ e as variáveis turbulentas $\kappa, \varepsilon$ e $\nu_{t}$ são calculadas no centro das células computacionais e as componentes de velocidade $u, v$ e $w$ nas faces $\left(i+\frac{1}{2}, j, k\right),\left(i, j+\frac{1}{2}, k\right)$ e $\left(i, j, k+\frac{1}{2}\right)$ dessas células, respectivamente. A título de exemplo, a seguir são apresentadas as discretizações, em diferentes posições da malha, de três termos difusivos que aparecem nas equações (2.64)-(2.68).

$$
\begin{aligned}
\left.\frac{\partial}{\partial x}\left(\frac{\partial w}{\partial x}-\frac{\partial u}{\partial z}\right)\right|_{i, j, k+\frac{1}{2}} ^{n} \approx & \frac{1}{\delta x}\left[\left.\left(\frac{\partial w}{\partial x}-\frac{\partial u}{\partial z}\right)\right|_{i+\frac{1}{2}, j, k+\frac{1}{2}} ^{n}-\left.\left(\frac{\partial w}{\partial x}-\frac{\partial u}{\partial z}\right)\right|_{i-\frac{1}{2}, j, k+\frac{1}{2}} ^{n}\right] \\
\approx & \frac{1}{\delta x}\left[\left(\frac{w_{i+1, j, k+\frac{1}{2}}^{n}-w_{i, j, k+\frac{1}{2}}^{n}}{\delta x}-\frac{u_{i+\frac{1}{2}, j, k+1}^{n}-u_{i+\frac{1}{2}, j, k}^{n}}{\delta z}\right)\right. \\
& \left.-\left(\frac{w_{i, j, k+\frac{1}{2}}^{n}-w_{i-1, j, k+\frac{1}{2}}^{n}}{\delta x}-\frac{u_{i-\frac{1}{2}, j, k+1}^{n}-u_{i-\frac{1}{2}, j, k}^{n}}{\delta z}\right)\right] \\
= & \frac{w_{i+1, j, k+\frac{1}{2}}^{n}-2 w_{i, j, k+\frac{1}{2}}^{n}+w_{i-1, j, k+\frac{1}{2}}^{n}}{\delta x^{2}}-u_{i-\frac{1}{2}, j, k}^{n}+u_{i-\frac{1}{2}, j, k+1}^{n} \\
& +\frac{u_{i+\frac{1}{2}, j, k}^{n}-u_{i+\frac{1}{2}, j, k+1}^{n}}{\delta x \delta z}
\end{aligned}
$$




$$
\begin{aligned}
\left.\frac{\partial}{\partial z}\left(\nu_{t}\left(\frac{\partial u}{\partial z}+\frac{\partial w}{\partial x}\right)\right)\right|_{i+\frac{1}{2}, j, k} ^{n} \approx & \frac{1}{\delta z}\left[\left.\left(\nu_{t}\left(\frac{\partial u}{\partial z}+\frac{\partial w}{\partial x}\right)\right)\right|_{i+\frac{1}{2}, j, k+\frac{1}{2}} ^{n}-\left.\left(\nu_{t}\left(\frac{\partial u}{\partial z}+\frac{\partial w}{\partial x}\right)\right)\right|_{i+\frac{1}{2}, j, k-\frac{1}{2}} ^{n}\right] \\
\approx & \frac{1}{\delta z}\left[\nu_{t+\frac{1}{2}, j, k+\frac{1}{2}}^{n}\left(\frac{u_{i+\frac{1}{2}, j, k+1}^{n}-u_{i+\frac{1}{2}, j, k}^{n}}{\delta z}+\frac{w_{i+1, j, k+\frac{1}{2}}^{n}-w_{i+1, j, k+\frac{1}{2}}^{n}}{\delta x}\right)\right. \\
& \left.-\nu_{t+\frac{1}{2}, j, k-\frac{1}{2}}^{n}\left(\frac{u_{i+\frac{1}{2}, j, k}^{n}-u_{i+\frac{1}{2}, j, k-1}^{n}}{\delta z}+\frac{w_{i+1, j, k-\frac{1}{2}}^{n}-w_{i, j, k-\frac{1}{2}}^{n}}{\delta x}\right)\right],
\end{aligned}
$$

em que

$$
\nu_{t i+\frac{1}{2}, j, k+\frac{1}{2}}^{n} \approx 0.25\left(\nu_{t i, j, k}^{n}+\nu_{t i, j, k+1}^{n}+\nu_{t i+1, j, k}^{n}+\nu_{t i+1, j, k+1}^{n}\right)
$$

e

$$
\begin{aligned}
\nu_{t_{i+\frac{1}{2}, j, k-\frac{1}{2}}}^{n} \approx & 0.25\left(\nu_{t i, j, k-1}^{n}+\nu_{t i, j, k}^{n}+\nu_{t i+1, j, k-1}^{n}+\nu_{t i+1, j, k}^{n}\right) \\
\left.\frac{\partial}{\partial x}\left(\left(1+\frac{\nu_{t}}{\sigma_{\kappa}}\right) \frac{\partial \kappa}{\partial x}\right)\right|_{i, j, k} ^{n} \approx & \frac{1}{\delta x}\left[\left.\left(1+\frac{\nu_{t}}{\sigma_{\kappa}}\right)\left(\frac{\partial \kappa}{\partial x}\right)\right|_{i+\frac{1}{2}, j, k} ^{n}-\left.\left(1+\frac{\nu_{t}}{\sigma_{\kappa}}\right)\left(\frac{\partial \kappa}{\partial x}\right)\right|_{i-\frac{1}{2}, j, k} ^{n}\right] \\
\approx & \frac{1}{\delta x}\left[\left(1+\frac{\nu_{t i+\frac{1}{2}, j, k}^{n}}{\sigma_{\kappa}}\right)\left(\frac{\kappa_{i+1, j, k}^{n}-\kappa_{i, j, k}^{n}}{\delta x}\right)\right. \\
& \left.-\left(1+\frac{\nu_{t i-\frac{1}{2}, j, k}^{n}}{\sigma_{\kappa}}\right)\left(\frac{\kappa_{i, j, k}^{n}-\kappa_{i-1, j, k}^{n}}{\delta x}\right)\right]
\end{aligned}
$$

em que

$$
\nu_{t i+\frac{1}{2}, j, k}^{n} \approx 0.5\left(\nu_{t i+1, j, k}^{n}+\nu_{t i, j, k}^{n}\right)
$$

e

$$
\nu_{t_{i-\frac{1}{2}, j, k}}^{n} \approx 0.5\left(\nu_{t i, j, k}^{n}+\nu_{t i-1, j, k}^{n}\right) .
$$

Outros termos difusivos são aproximados de maneira análogo.

Os termos de produção de energia cinética turbulenta dados em (2.56) são aproximados também por diferenças centrais. Por exemplo, o último termo desta equação é aproximados no ponto $\mathcal{P}=(i, j, k)$ por

$$
\begin{aligned}
\left.\left(\frac{\partial v}{\partial z}+\frac{\partial w}{\partial y}\right)^{2}\right|_{i, j, k} ^{n} \approx & \left(\frac{v_{i, j, k+\frac{1}{2}}^{n}-v_{i, j, k-\frac{1}{2}}^{n}}{\delta z}+\frac{w_{i, j+\frac{1}{2}, k}^{n}-w_{i, j-\frac{1}{2}, k}^{n}}{\delta y}\right)^{2} \\
\approx & {\left[\left(\frac{v_{i, j+\frac{1}{2}, k+1}^{n}+v_{i, j-\frac{1}{2}, k+1}^{n}-v_{i, j+\frac{1}{2}, k-1}^{n}-v_{i, j-\frac{1}{2}, k-1}^{n}}{4 \delta z}\right)\right.} \\
& \left.+\left(\frac{w_{i, j+1, k+\frac{1}{2}}^{n}+w_{i, j+1, k-\frac{1}{2}}^{n}-w_{i, j-1, k+\frac{1}{2}}^{n}-w_{i, j-1, k-\frac{1}{2}}^{n}}{4 \delta y}\right)\right]^{2}
\end{aligned}
$$


A escala de tempo $T_{t}$ e a viscosidade turbulenta $\nu_{t}$ dados por (2.54) e (2.55) são aproximados no ponto $\mathcal{P}=(i, j, k)$ como

$$
T_{t i, j, k}^{n}=\frac{\kappa_{i, j, k}^{n}}{\varepsilon_{i, j, k}^{n}} .
$$

e

$$
\nu_{t i, j, k}^{n}=C_{\mu} \kappa_{i, j, k}^{n} T_{t i, j, k}^{n}
$$

\subsubsection{Discretização dos Termos Advectivos}

Nesta subseção, apresentam-se as discretizações dos termos advectivos não lineares que aparecem nas equações de conservação (2.64)-(2.68). A partir de agora, as propriedades $u, v$ e $w$ (casos instantâneo e médio), $\kappa$ e $\varepsilon$ avaliadas nas faces $f$ e $g$ (como visto na Figura 3.2) são calculadas utilizando-se o esquema "upwind" de alta resolução ADBQUICKEST estudado na seção 3.5.

Por simplicidade e sem perda de generalidade, somente os termos advectivos da equação (2.64) serão discretizados e apresentados aqui.

Considera-se a variável $\phi$ na aproximação (3.1) como sendo a componente $u$ da velocidade $(\phi=u)$ e a posição $\mathcal{P}=\left(i+\frac{1}{2}, j, k\right)$ em que o termo $-C O N V(u)$ é avaliado, isto é,

$$
\begin{aligned}
\left.\left(\frac{\partial(u u)}{\partial x}+\frac{\partial(u v)}{\partial y}+\frac{\partial(u w)}{\partial z}\right)\right|_{i+\frac{1}{2}, j, k} \approx & \frac{\bar{u}_{1} u_{i+1, j, k}-\bar{u}_{2} u_{i, j, k}}{\delta x}+\frac{\bar{v}_{1} u_{i+\frac{1}{2}, j+\frac{1}{2}, k}-\bar{v}_{2} u_{i+\frac{1}{2}, j-\frac{1}{2}, k}}{\delta y} \\
& +\frac{\bar{w}_{1} u_{i+\frac{1}{2}, j, k+\frac{1}{2}}-\bar{w}_{2} u_{i+\frac{1}{2}, j, k-\frac{1}{2}}}{\delta z}
\end{aligned}
$$

Os valores das velocidades advectivas $\bar{u}_{1}, \bar{u}_{2}, \bar{v}_{1}, \bar{v}_{2}, \bar{w}_{1}$ e $\bar{w}_{2}$ em (4.20) são obtidos utilizando-se médias. Por exemplo, os valores $\bar{v}_{1}$ e $\bar{v}_{2}$ (as outras velocidades advectivas são calculadas de forma análoga) são aproximados por

$$
\bar{v}_{1}=\bar{v}_{i+\frac{1}{2}, j+\frac{1}{2}, k} \approx 0.5\left(v_{i+1, j+\frac{1}{2}, k}+v_{i, j+\frac{1}{2}, k}\right)
$$

$\mathrm{e}$

$$
\bar{v}_{2}=\bar{v}_{i+\frac{1}{2}, j-\frac{1}{2}, k} \approx 0.5\left(v_{i+1, j-\frac{1}{2}, k}+v_{i, j-\frac{1}{2}, k}\right) .
$$

Na equação (4.20), os valores da propriedade convectada $u$ nos pontos $(i, j, k),(i+1, j, k)$, $\left(i+\frac{1}{2}, j+\frac{1}{2}, k\right),\left(i+\frac{1}{2}, j-\frac{1}{2}, k\right),\left(i+\frac{1}{2}, j, k+\frac{1}{2}\right)$ e $\left(i+\frac{1}{2}, j, k-\frac{1}{2}\right)$ são determinados utilizando-se a estratégia "upwind" de alta resolução ADBQUICKEST dado pela equação (3.22).

Uma vez conhecidas as direções das velocidades advectivas $\bar{u}_{1}, \bar{u}_{2}, \bar{v}_{1}, \bar{v}_{2}, \bar{w}_{1}$ e $\bar{w}_{2}$ nas faces $(i, j, k),(i+1, j, k),\left(i+\frac{1}{2}, j+\frac{1}{2}, k\right),\left(i+\frac{1}{2}, j-\frac{1}{2}, k\right),\left(i+\frac{1}{2}, j, k+\frac{1}{2}\right)$ e $\left(i+\frac{1}{2}, j, k-\frac{1}{2}\right)$, respectivamente, os pontos vizinhos a jusante $\mathbf{D}$, a montante $\mathbf{U}$ e remoto a montante $\mathbf{R}$, em cada caso, ficam automaticamente especificados. Dessa forma, usando-se as definições de Leonard em (3.8), as aproximações para as velocidades convectadas nessas faces são determinadas e implementadas 
no código Freeflow 3D como segue:

(1) Aproximações para $u_{i+1, j, k}$ :

- Quando $\bar{u}_{1}=u_{i+1, j, k}>0$ e $\hat{u}_{i+\frac{1}{2}, j, k}=\frac{u_{i+\frac{1}{2}, j, k}-u_{i-\frac{1}{2}, j, k}}{u_{i+\frac{3}{2}, j, k}-u_{i-\frac{1}{2}, j, k}}$ tem-se que

$$
u_{i+1, j, k}=\left\{\begin{array}{lll}
u_{i+\frac{1}{2}, j, k}, & \text { se } & \hat{u}_{i+\frac{1}{2}, j, k} \notin[0,1] \\
(2-\theta) u_{i+\frac{1}{2}, j, k}-(1-\theta) u_{i-\frac{1}{2}, j, k}, & \text { se } 0<\hat{u}_{i+\frac{1}{2}, j, k}<a \\
\alpha_{D} u_{i+\frac{3}{2}, j, k}+\alpha_{U} u_{i+\frac{1}{2}, j, k}-\alpha_{R} u_{i-\frac{1}{2}, j, k}, & \text { se } a \leqslant \hat{u}_{i+\frac{1}{2}, j, k} \leqslant b \\
(1-\theta) u_{i+\frac{3}{2}, j, k}+\theta u_{i+\frac{1}{2}, j, k}, & \text { se } b<\hat{u}_{i+\frac{1}{2}, j, k}<1
\end{array}\right.
$$

na qual

$$
\mathbf{D}=\left(i+\frac{3}{2}, j, k\right), \quad \mathbf{U}=\left(i+\frac{1}{2}, j, k\right), \quad \mathbf{R}=\left(i-\frac{1}{2}, j, k\right)
$$

- Quando $\bar{u}_{1}=u_{i+1, j, k}<0$ e $\hat{u}_{i+\frac{3}{2}, j, k}=\frac{u_{i+\frac{3}{2}, j, k}-u_{i+\frac{5}{2}, j, k}}{u_{i+\frac{1}{2}, j, k}-u_{i+\frac{5}{2}, j, k}}$ tem-se que

$$
u_{i+1, j, k}=\left\{\begin{array}{lll}
u_{i+\frac{3}{2}, j, k}, & \text { se } \hat{u}_{i+\frac{3}{2}, j, k} \notin[0,1], \\
(2-\theta) u_{i+\frac{3}{2}, j, k}-(1-\theta) u_{i+\frac{5}{2}, j, k}, & \text { se } 0<\hat{u}_{i+\frac{3}{2}, j, k}<a, \\
\alpha_{D} u_{i+\frac{1}{2}, j, k}+\alpha_{U} u_{i+\frac{3}{2}, j, k}-\alpha_{R} u_{i+\frac{5}{2}, j, k}, & \text { se } a \leqslant \hat{u}_{i+\frac{3}{2}, j, k} \leqslant b, \\
(1-\theta) u_{i+\frac{1}{2}, j, k}+\theta u_{i+\frac{3}{2}, j, k}, & \text { se } b<\hat{u}_{i+\frac{3}{2}, j, k}<1
\end{array}\right.
$$

na qual

$$
\mathbf{D}=\left(i+\frac{1}{2}, j, k\right), \quad \mathbf{U}=\left(i+\frac{3}{2}, j, k\right), \quad \mathbf{R}=\left(i+\frac{5}{2}, j, k\right)
$$

(2) Aproximações para $u_{i, j, k}$ :

- Quando $\bar{u}_{2}=u_{i, j, k}>0$ e $\hat{u}_{i-\frac{1}{2}, j, k}=\frac{u_{i-\frac{1}{2}, j, k}-u_{i-\frac{3}{2}, j, k}}{u_{i+\frac{1}{2}, j, k}-u_{i-\frac{3}{2}, j, k}}$ tem-se que

$$
u_{i, j, k}= \begin{cases}u_{i-\frac{1}{2}, j, k}, & \text { se } \hat{u}_{i-\frac{1}{2}, j, k} \notin[0,1] \\ (2-\theta) u_{i-\frac{1}{2}, j, k}-(1-\theta) u_{i-\frac{3}{2}, j, k}, & \text { se } 0<\hat{u}_{i-\frac{1}{2}, j, k}<a \\ \alpha_{D} u_{i+\frac{1}{2}, j, k}+\alpha_{U} u_{i-\frac{1}{2}, j, k}-\alpha_{R} u_{i-\frac{3}{2}, j, k}, & \text { se } a \leqslant \hat{u}_{i-\frac{1}{2}, j, k} \leqslant b \\ (1-\theta) u_{i+\frac{1}{2}, j, k}+\theta u_{i-\frac{1}{2}, j, k}, & \text { se } b<\hat{u}_{i-\frac{1}{2}, j, k}<1\end{cases}
$$

na qual

$$
\mathbf{D}=\left(i+\frac{1}{2}, j, k\right), \quad \mathbf{U}=\left(i-\frac{1}{2}, j, k\right), \quad \mathbf{R}=\left(i-\frac{3}{2}, j, k\right)
$$


- Quando $\bar{u}_{2}=u_{i, j, k}<0$ e $\hat{u}_{i+\frac{1}{2}, j, k}=\frac{u_{i+\frac{1}{2}, j, k}-u_{i+\frac{3}{2}, j, k}}{u_{i-\frac{1}{2}, j, k}-u_{i+\frac{3}{2}, j, k}}$ tem-se que

$$
u_{i, j, k}= \begin{cases}u_{i-\frac{1}{2}, j, k}, & \text { se } \hat{u}_{i+\frac{1}{2}, j, k} \notin[0,1], \\ (2-\theta) u_{i+\frac{1}{2}, j, k}-(1-\theta) u_{i+\frac{3}{2}, j, k}, & \text { se } 0<\hat{u}_{i+\frac{1}{2}, j, k}<a, \\ \alpha_{D} u_{i-\frac{1}{2}, j, k}+\alpha_{U} u_{i+\frac{1}{2}, j, k}-\alpha_{R} u_{i+\frac{3}{2}, j, k}, & \text { se } a \leqslant \hat{u}_{i+\frac{1}{2}, j, k} \leqslant b \\ (1-\theta) u_{i-\frac{1}{2}, j, k}+\theta u_{i+\frac{1}{2}, j, k}, & \text { se } b<\hat{u}_{i+\frac{1}{2}, j, k}<1\end{cases}
$$

na qual

$$
\mathbf{D}=\left(i-\frac{1}{2}, j, k\right), \quad \mathbf{U}=\left(i+\frac{1}{2}, j, k\right), \quad \mathbf{R}=\left(i+\frac{3}{2}, j, k\right) ;
$$

(3) Aproximações para $u_{i+\frac{1}{2}, j+\frac{1}{2}, k}$ :

- Quando $\bar{v}_{1}=v_{i+\frac{1}{2}, j+\frac{1}{2}, k}>0$ e $\hat{u}_{i+\frac{1}{2}, j, k}=\frac{u_{i+\frac{1}{2}, j, k}-u_{i+\frac{1}{2}, j-1, k}}{u_{i+\frac{1}{2}, j+1, k}-u_{i+\frac{1}{2}, j-1, k}}$ tem-se que

$$
u_{i+\frac{1}{2}, j+\frac{1}{2}, k}= \begin{cases}u_{i+\frac{1}{2}, j, k}, & \text { se } \hat{u}_{i+\frac{1}{2}, j, k} \notin[0,1], \\ (2-\theta) u_{i+\frac{1}{2}, j, k}-(1-\theta) u_{i+\frac{1}{2}, j-1, k}, & \text { se } 0<\hat{u}_{i+\frac{1}{2}, j, k}<a \\ \alpha_{D} u_{i+\frac{1}{2}, j+1, k}+\alpha_{U} u_{i+\frac{1}{2}, j, k}-\alpha_{R} u_{i+\frac{1}{2}, j-1, k}, & \text { se } a \leqslant \hat{u}_{i+\frac{1}{2}, j, k} \leqslant b \\ (1-\theta) u_{i+\frac{1}{2}, j+1, k}+\theta u_{i+\frac{1}{2}, j, k}, & \text { se } b<\hat{u}_{i+\frac{1}{2}, j, k}<1,\end{cases}
$$

na qual

$$
\mathbf{D}=\left(i+\frac{1}{2}, j+1, k\right), \quad \mathbf{U}=\left(i+\frac{1}{2}, j, k\right), \quad \mathbf{R}=\left(i+\frac{1}{2}, j-1, k\right) ;
$$

- Quando $\bar{v}_{1}=v_{i+\frac{1}{2}, j+\frac{1}{2}, k}<0$ e $\hat{u}_{i+\frac{1}{2}, j+1, k}=\frac{u_{i+\frac{1}{2}, j+1, k}-u_{i+\frac{1}{2}, j+2, k}}{u_{i+\frac{1}{2}, j, k}-u_{i+\frac{1}{2}, j+2, k}}$ tem-se que

$$
u_{i+\frac{1}{2}, j+\frac{1}{2}, k}= \begin{cases}u_{i+\frac{1}{2}, j+1, k}, & \text { se } \hat{u}_{i+\frac{1}{2}, j+1, k} \notin[0,1], \\ (2-\theta) u_{i+\frac{1}{2}, j+1, k}-(1-\theta) u_{i+\frac{1}{2}, j+2, k}, & \text { se } 0<\hat{u}_{i+\frac{1}{2}, j+1, k}<a, \\ \alpha_{D} u_{i+\frac{1}{2}, j, k}+\alpha_{U} u_{i+\frac{1}{2}, j+1, k}-\alpha_{R} u_{i+\frac{1}{2}, j+2, k}, & \text { se } a \leqslant \hat{u}_{i+\frac{1}{2}, j+1, k} \leqslant b, \\ (1-\theta) u_{i+\frac{1}{2}, j, k}+\theta u_{i+\frac{1}{2}, j+1, k}, & \text { se } b<\hat{u}_{i+\frac{1}{2}, j+1, k}<1,\end{cases}
$$

na qual

$$
\mathbf{D}=\left(i+\frac{1}{2}, j, k\right), \quad \mathbf{U}=\left(i+\frac{1}{2}, j+1, k\right), \quad \mathbf{R}=\left(i+\frac{1}{2}, j+2, k\right) ;
$$

(4) Aproximações para $u_{i+\frac{1}{2}, j-\frac{1}{2}, k}$ : 
- Quando $\bar{v}_{2}=v_{i+\frac{1}{2}, j-\frac{1}{2}, k}>0$ e $\hat{u}_{i+\frac{1}{2}, j-1, k}=\frac{u_{i+\frac{1}{2}, j-1, k}-u_{i+\frac{1}{2}, j-2, k}}{u_{i+\frac{1}{2}, j, k}-u_{i+\frac{1}{2}, j-2, k}}$ tem-se que

$$
u_{i+\frac{1}{2}, j-\frac{1}{2}, k}= \begin{cases}u_{i+\frac{1}{2}, j-1, k}, & \text { se } \hat{u}_{i+\frac{1}{2}, j-1, k} \notin[0,1], \\ (2-\theta) u_{i+\frac{1}{2}, j-1, k}-(1-\theta) u_{i+\frac{1}{2}, j-2, k}, & \text { se } 0<\hat{u}_{i+\frac{1}{2}, j+1, k}<a, \\ \alpha_{D} u_{i+\frac{1}{2}, j, k}+\alpha_{U} u_{i+\frac{1}{2}, j-1, k}-\alpha_{R} u_{i+\frac{1}{2}, j-2, k}, & \text { se } a \leqslant \hat{u}_{i+\frac{1}{2}, j-1, k} \leqslant b, \\ (1-\theta) u_{i+\frac{1}{2}, j, k}+\theta u_{i+\frac{1}{2}, j-1, k}, & \text { se } b<\hat{u}_{i+\frac{1}{2}, j-1, k}<1,\end{cases}
$$

na qual

$$
\mathbf{D}=\left(i+\frac{1}{2}, j, k\right), \quad \mathbf{U}=\left(i+\frac{1}{2}, j-1, k\right), \quad \mathbf{R}=\left(i+\frac{1}{2}, j-2, k\right)
$$

- Quando $\bar{v}_{2}=v_{i+\frac{1}{2}, j-\frac{1}{2}, k}<0$ e $\hat{u}_{i+\frac{1}{2}, j, k}=\frac{u_{i+\frac{1}{2}, j, k}-u_{i+\frac{1}{2}, j+1, k}}{u_{i+\frac{1}{2}, j-1, k}-u_{i+\frac{1}{2}, j+1, k}}$ tem-se que

$$
u_{i+\frac{1}{2}, j-\frac{1}{2}, k}= \begin{cases}u_{i+\frac{1}{2}, j, k}, & \text { se } \quad \hat{u}_{i+\frac{1}{2}, j, k} \notin[0,1], \\ (2-\theta) u_{i+\frac{1}{2}, j, k}-(1-\theta) u_{i+\frac{1}{2}, j+1, k}, & \text { se } 0<\hat{u}_{i+\frac{1}{2}, j, k}<a, \\ \alpha_{D} u_{i+\frac{1}{2}, j-1, k}+\alpha_{U} u_{i+\frac{1}{2}, j, k}-\alpha_{R} u_{i+\frac{1}{2}, j+1, k}, & \text { se } a \leqslant \hat{u}_{i+\frac{1}{2}, j, k} \leqslant b, \\ (1-\theta) u_{i+\frac{1}{2}, j-1, k}+\theta u_{i+\frac{1}{2}, j, k}, & \text { se } b<\hat{u}_{i+\frac{1}{2}, j, k}<1,\end{cases}
$$

na qual

$$
\mathbf{D}=\left(i+\frac{1}{2}, j-1, k\right), \quad \mathbf{U}=\left(i+\frac{1}{2}, j, k\right), \quad \mathbf{R}=\left(i+\frac{1}{2}, j+1, k\right)
$$

(5) Aproximações para $u_{i+\frac{1}{2}, j, k+\frac{1}{2}}$ :

- Quando $\bar{w}_{1}=w_{i+\frac{1}{2}, j, k+\frac{1}{2}}>0$ e $\hat{u}_{i+\frac{1}{2}, j, k}=\frac{u_{i+\frac{1}{2}, j, k}-u_{i+\frac{1}{2}, j, k-1}}{u_{i+\frac{1}{2}, j, k+1}-u_{i+\frac{1}{2}, j, k-1}}$ tem-se que

$$
u_{i+\frac{1}{2}, j, k+\frac{1}{2}}= \begin{cases}u_{i+\frac{1}{2}, j, k}, & \text { se } \quad \hat{u}_{i+\frac{1}{2}, j, k} \notin[0,1], \\ (2-\theta) u_{i+\frac{1}{2}, j, k}-(1-\theta) u_{i+\frac{1}{2}, j, k-1}, & \text { se } 0<\hat{u}_{i+\frac{1}{2}, j, k}<a \\ \alpha_{D} u_{i+\frac{1}{2}, j, k+1}+\alpha_{U} u_{i+\frac{1}{2}, j, k}-\alpha_{R} u_{i+\frac{1}{2}, j, k-1}, & \text { se } a \leqslant \hat{u}_{i+\frac{1}{2}, j, k} \leqslant b \\ (1-\theta) u_{i+\frac{1}{2}, j, k+1}+\theta u_{i+\frac{1}{2}, j, k}, & \text { se } b<\hat{u}_{i+\frac{1}{2}, j, k}<1\end{cases}
$$

na qual

$$
\mathbf{D}=\left(i+\frac{1}{2}, j, k+1\right), \quad \mathbf{U}=\left(i+\frac{1}{2}, j, k\right), \quad \mathbf{R}=\left(i+\frac{1}{2}, j, k-1\right)
$$


- Quando $\bar{w}_{1}=w_{i+\frac{1}{2}, j, k+\frac{1}{2}}<0$ e $\hat{u}_{i+\frac{1}{2}, j, k+1}=\frac{u_{i+\frac{1}{2}, j, k+1}-u_{i+\frac{1}{2}, j, k+2}}{u_{i+\frac{1}{2}, j, k}-u_{i+\frac{1}{2}, j, k+2}}$ tem-se que

$$
u_{i+\frac{1}{2}, j, k+\frac{1}{2}}= \begin{cases}u_{i+\frac{1}{2}, j, k+1}, & \text { se } \hat{u}_{i+\frac{1}{2}, j, k+1} \notin[0,1], \\ (2-\theta) u_{i+\frac{1}{2}, j, k+1}-(1-\theta) u_{i+\frac{1}{2}, j, k+2}, & \text { se } 0<\hat{u}_{i+\frac{1}{2}, j, k+1}<a, \\ \alpha_{D} u_{i+\frac{1}{2}, j, k}+\alpha_{U} u_{i+\frac{1}{2}, j, k+1}-\alpha_{R} u_{i+\frac{1}{2}, j, k+2}, & \text { se } a \leqslant \hat{u}_{i+\frac{1}{2}, j, k+1} \leqslant b, \\ (1-\theta) u_{i+\frac{1}{2}, j, k}+\theta u_{i+\frac{1}{2}, j, k+1}, & \text { se } b<\hat{u}_{i+\frac{1}{2}, j, k+1}<1,\end{cases}
$$

na qual

$$
\mathbf{D}=\left(i+\frac{1}{2}, j, k\right), \quad \mathbf{U}=\left(i+\frac{1}{2}, j, k+1\right), \quad \mathbf{R}=\left(i+\frac{1}{2}, j, k+2\right)
$$

(6) Aproximações para $u_{i+\frac{1}{2}, j, k-\frac{1}{2}}$ :

- Quando $\bar{w}_{2}=w_{i+\frac{1}{2}, j, k-\frac{1}{2}}>0$ e $\hat{u}_{i+\frac{1}{2}, j, k-1}=\frac{u_{i+\frac{1}{2}, j, k-1}-u_{i+\frac{1}{2}, j, k-2}}{u_{i+\frac{1}{2}, j, k}-u_{i+\frac{1}{2}, j, k-2}}$ tem-se que

$$
u_{i+\frac{1}{2}, j, k-\frac{1}{2}}= \begin{cases}u_{i+\frac{1}{2}, j, k-1}, & \text { se } \hat{u}_{i+\frac{1}{2}, j, k-1} \notin[0,1], \\ (2-\theta) u_{i+\frac{1}{2}, j, k-1}-(1-\theta) u_{i+\frac{1}{2}, j, k-2}, & \text { se } 0<\hat{u}_{i+\frac{1}{2}, j, k-1}<a, \\ \alpha_{D} u_{i+\frac{1}{2}, j, k}+\alpha_{U} u_{i+\frac{1}{2}, j, k-1}-\alpha_{R} u_{i+\frac{1}{2}, j, k-2}, & \text { se } a \leqslant \hat{u}_{i+\frac{1}{2}, j, k-1} \leqslant b, \\ (1-\theta) u_{i+\frac{1}{2}, j, k}+\theta u_{i+\frac{1}{2}, j, k-1}, & \text { se } b<\hat{u}_{i+\frac{1}{2}, j, k-1}<1,\end{cases}
$$

na qual

$$
\mathbf{D}=\left(i+\frac{1}{2}, j, k\right), \quad \mathbf{U}=\left(i+\frac{1}{2}, j, k-1\right), \quad \mathbf{R}=\left(i+\frac{1}{2}, j, k-2\right) ;
$$

- Quando $\bar{w}_{2}=w_{i+\frac{1}{2}, j, k-\frac{1}{2}}<0$ e $\hat{u}_{i+\frac{1}{2}, j, k}=\frac{u_{i+\frac{1}{2}, j, k}-u_{i+\frac{1}{2}, j, k+1}}{u_{i+\frac{1}{2}, j, k-1}-u_{i+\frac{1}{2}, j, k+1}}$ tem-se que

$$
u_{i+\frac{1}{2}, j, k-\frac{1}{2}}= \begin{cases}u_{i+\frac{1}{2}, j, k}, & \text { se } \hat{u}_{i+\frac{1}{2}, j, k} \notin[0,1], \\ (2-\theta) u_{i+\frac{1}{2}, j, k}-(1-\theta) u_{i+\frac{1}{2}, j, k+1}, & \text { se } 0<\hat{u}_{i+\frac{1}{2}, j, k}<a, \\ \alpha_{D} u_{i+\frac{1}{2}, j, k-1}+\alpha_{U} u_{i+\frac{1}{2}, j, k}-\alpha_{R} u_{i+\frac{1}{2}, j, k+1}, & \text { se } a \leqslant \hat{u}_{i+\frac{1}{2}, j, k} \leqslant b, \\ (1-\theta) u_{i+\frac{1}{2}, j, k-1}+\theta u_{i+\frac{1}{2}, j, k}, & \text { se } b<\hat{u}_{i+\frac{1}{2}, j, k}<1,\end{cases}
$$

na qual

$$
\mathbf{D}=\left(i+\frac{1}{2}, j, k-1\right), \quad \mathbf{U}=\left(i+\frac{1}{2}, j, k\right), \quad \mathbf{R}=\left(i+\frac{1}{2}, j, k+1\right)
$$

A discretização dos outros termos advectivos das equações de conservação (2.65) e (2.66) é feita de forma análoga. 
Considerando agora, a variável transportada como sendo a variável turbulenta $\kappa$, a aproximação para o termo advectivo da equação de conservação $(2.67)$ aplicada no ponto $\mathcal{P}=(i, j, k)$ é dada por

$$
\begin{aligned}
\left.\left(\frac{\partial(\kappa u)}{\partial x}+\frac{\partial(\kappa v)}{\partial y}+\frac{\partial(\kappa w)}{\partial z}\right)\right|_{i, j, k} \approx & \frac{\bar{u}_{1} \kappa_{i+\frac{1}{2}, j, k}-\bar{u}_{2} \kappa_{i-\frac{1}{2}, j, k}}{\delta x}+\frac{\bar{v}_{1} \kappa_{i, j+\frac{1}{2}, k}-\bar{v}_{2} \kappa_{i, j-\frac{1}{2}, k}}{\delta y} \\
& +\frac{\bar{w}_{1} \kappa_{i, j, k+\frac{1}{2}}-\bar{w}_{2} \kappa_{i, j, k-\frac{1}{2}}}{\delta z}
\end{aligned}
$$

em que os valores $\bar{u}_{1}, \bar{u}_{2}, \bar{v}_{1}, \bar{v}_{2}, \bar{w}_{1}$ e $\bar{w}_{2}$ são dados, respectivamente, por

$$
\begin{array}{ccc}
\bar{u}_{1}=u_{i+\frac{1}{2}, j, k} & \text { e } & \bar{u}_{2}=u_{i-\frac{1}{2}, j, k} \\
\bar{v}_{1}=u_{i, j+\frac{1}{2}, k} & \text { e } & \bar{v}_{2}=u_{i, j-\frac{1}{2}, k} \\
\bar{w}_{1}=u_{i, j, k+\frac{1}{2}} & \text { e } & \bar{w}_{2}=u_{i, j, k-\frac{1}{2}}
\end{array}
$$

As aproximações para as propriedades $\kappa_{i+\frac{1}{2}, j, k}, \kappa_{i-\frac{1}{2}, j, k}, \kappa_{i, j+\frac{1}{2}, k}, \kappa_{i, j-\frac{1}{2}, k}, \kappa_{i, j, k+\frac{1}{2}}$ e $\kappa_{i, j, k-\frac{1}{2}}$ dadas em (4.21) são implementadas como segue:

(1) Aproximações para $\kappa_{i+\frac{1}{2}, j, k}$ :

- Quando $\bar{u}_{1}=u_{i+\frac{1}{2}, j, k}>0$ e $\hat{\kappa}_{i, j, k}=\frac{\kappa_{i, j, k}-\kappa_{i-1, j, k}}{\kappa_{i+1, j, k}-\kappa_{i-1, j, k}}$ tem-se que

$$
\kappa_{i+\frac{1}{2}, j, k}=\left\{\begin{array}{lll}
\kappa_{i, j, k}, & \text { se } \quad \hat{\kappa}_{i, j, k} \notin[0,1], \\
(2-\theta) \kappa_{i, j, k}-(1-\theta) \kappa_{i-1, j, k}, & \text { se } 0<\hat{\kappa}_{i, j, k}<a, \\
\alpha_{D} \kappa_{i+1, j, k}+\alpha_{U} \kappa_{i, j, k}-\alpha_{R} \kappa_{i-1, j, k}, & \text { se } a \leqslant \hat{\kappa}_{i, j, k} \leqslant b, \\
(1-\theta) \kappa_{i+1, j, k}+\theta \kappa_{i, j, k}, & \text { se } b<\hat{\kappa}_{i, j, k}<1,
\end{array}\right.
$$

na qual

$$
\mathbf{D}=(i+1, j, k), \quad \mathbf{U}=(i, j, k), \quad \mathbf{R}=(i-1, j, k)
$$

- Quando $\bar{u}_{1}=u_{i+\frac{1}{2}, j, k}<0$ e $\hat{\kappa}_{i+1, j, k}=\frac{\kappa_{i+1, j, k}-\kappa_{i+2, j, k}}{\kappa_{i, j, k}-\kappa_{i+2, j, k}}$ tem-se que

$$
\kappa_{i+\frac{1}{2}, j, k}=\left\{\begin{array}{lll}
\kappa_{i+1, j, k}, & \text { se } & \hat{\kappa}_{i+1, j, k} \notin[0,1], \\
(2-\theta) \kappa_{i+1, j, k}-(1-\theta) \kappa_{i+2, j, k}, & \text { se } 0<\hat{\kappa}_{i+1, j, k}<a, \\
\alpha_{D} \kappa_{i, j, k}+\alpha_{U} \kappa_{i+1, j, k}-\alpha_{R} \kappa_{i+2, j, k}, & \text { se } a \leqslant \hat{\kappa}_{i+1, j, k} \leqslant b, \\
(1-\theta) \kappa_{i, j, k}+\theta \kappa_{i+1, j, k}, & \text { se } b<\hat{\kappa}_{i+1, j, k}<1
\end{array}\right.
$$

na qual

$$
\mathbf{D}=(i, j, k), \quad \mathbf{U}=(i+1, j, k), \quad \mathbf{R}=(i+2, j, k)
$$


(2) Aproximações para $\kappa_{i-\frac{1}{2}, j, k}$ :

- Quando $\bar{u}_{2}=u_{i-\frac{1}{2}, j, k}>0$ e $\hat{\kappa}_{i-1, j, k}=\frac{\kappa_{i-1, j, k}-\kappa_{i-2, j, k}}{\kappa_{i, j, k}-\kappa_{i-2, j, k}}$ tem-se que

$$
\kappa_{i-\frac{1}{2}, j, k}= \begin{cases}\kappa_{i-1, j, k}, & \text { se } \hat{\kappa}_{i-1, j, k} \notin[0,1] \\ (2-\theta) \kappa_{i-1, j, k}-(1-\theta) \kappa_{i-2, j, k}, & \text { se } 0<\hat{\kappa}_{i-1, j, k}<a \\ \alpha_{D} \kappa_{i, j, k}+\alpha_{U} \kappa_{i-1, j, k}-\alpha_{R} \kappa_{i-2, j, k}, & \text { se } a \leqslant \hat{\kappa}_{i-1, j, k} \leqslant b \\ (1-\theta) \kappa_{i, j, k}+\theta \kappa_{i-1, j, k}, & \text { se } b<\hat{\kappa}_{i-1, j, k}<1\end{cases}
$$

na qual

$$
\mathbf{D}=(i, j, k), \quad \mathbf{U}=(i-1, j, k), \quad \mathbf{R}=(i-2, j, k)
$$

- Quando $\bar{u}_{2}=u_{i-\frac{1}{2}, j, k}<0$ e $\hat{\kappa}_{i, j, k}=\frac{\kappa_{i, j, k}-\kappa_{i+1, j, k}}{\kappa_{i-1, j, k}-\kappa_{i+1, j, k}}$ tem-se que

$$
\kappa_{i-\frac{1}{2}, j, k}= \begin{cases}\kappa_{i, j, k}, & \text { se } \hat{\kappa}_{i, j, k} \notin[0,1], \\ (2-\theta) \kappa_{i, j, k}-(1-\theta) \kappa_{i+1, j, k}, & \text { se } 0<\hat{\kappa}_{i, j, k}<a, \\ \alpha_{D} \kappa_{i-1, j, k}+\alpha_{U} \kappa_{i, j, k}-\alpha_{R} \kappa_{i+1, j, k}, & \text { se } a \leqslant \hat{\kappa}_{i, j, k} \leqslant b, \\ (1-\theta) \kappa_{i-1, j, k}+\theta \kappa_{i, j, k}, & \text { se } b<\hat{\kappa}_{i, j, k}<1,\end{cases}
$$

na qual

$$
\mathbf{D}=(i-1, j, k), \quad \mathbf{U}=(i, j, k), \quad \mathbf{R}=(i+1, j, k)
$$

(3) Aproximações para $\kappa_{i, j+\frac{1}{2}, k}$ :

- Quando $\bar{v}_{1}=v_{i, j+\frac{1}{2}, k}>0$ e $\hat{\kappa}_{i, j, k}=\frac{\kappa_{i, j, k}-\kappa_{i, j-1, k}}{\kappa_{i, j+1, k}-\kappa_{i, j-1, k}}$ tem-se que

$$
\kappa_{i, j+\frac{1}{2}, k}= \begin{cases}\kappa_{i, j, k}, & \text { se } \hat{\kappa}_{i, j, k} \notin[0,1], \\ (2-\theta) \kappa_{i, j, k}-(1-\theta) \kappa_{i, j-1, k}, & \text { se } 0<\hat{\kappa}_{i, j, k}<a, \\ \alpha_{D} \kappa_{i, j+1, k}+\alpha_{U} \kappa_{i, j, k}-\alpha_{R} \kappa_{i, j-1, k}, & \text { se } a \leqslant \hat{\kappa}_{i, j, k} \leqslant b, \\ (1-\theta) \kappa_{i, j+1, k}+\theta \kappa_{i, j, k}, & \text { se } b<\hat{\kappa}_{i, j, k}<1,\end{cases}
$$

na qual

$$
\mathbf{D}=(i, j+1, k), \quad \mathbf{U}=(i, j, k), \quad \mathbf{R}=(i, j-1, k)
$$


- Quando $\bar{v}_{1}=v_{i, j+\frac{1}{2}, k}<0$ e $\hat{\kappa}_{i, j+1, k}=\frac{\kappa_{i, j+1, k}-\kappa_{i, j+2, k}}{\kappa_{i, j, k}-\kappa_{i, j+2, k}}$ tem-se que

$$
\kappa_{i, j+\frac{1}{2}, k}=\left\{\begin{array}{lll}
\kappa_{i, j+1, k}, & \text { se } \quad \hat{\kappa}_{i, j+1, k} \notin[0,1] \\
(2-\theta) \kappa_{i, j+1, k}-(1-\theta) \kappa_{i, j+2, k}, & \text { se } 0<\hat{\kappa}_{i, j+1, k}<a \\
\alpha_{D} \kappa_{i, j, k}+\alpha_{U} \kappa_{i, j+1, k}-\alpha_{R} \kappa_{i, j+2, k}, & \text { se } a \leqslant \hat{\kappa}_{i, j+1, k} \leqslant b \\
(1-\theta) \kappa_{i, j, k}+\theta \kappa_{i, j+1, k}, & \text { se } b<\hat{\kappa}_{i, j+1, k}<1
\end{array}\right.
$$

na qual

$$
\mathbf{D}=(i, j, k), \quad \mathbf{U}=(i, j+1, k), \quad \mathbf{R}=(i, j+2, k) ;
$$

(4) Aproximações para $\kappa_{i, j-\frac{1}{2}, k}$ :

- Quando $\bar{v}_{2}=v_{i, j-\frac{1}{2}, k}>0$ e $\hat{\kappa}_{i, j-1, k}=\frac{\kappa_{i, j-1, k}-\kappa_{i, j-2, k}}{\kappa_{i, j, k}-\kappa_{i, j-2, k}}$ tem-se que

$$
\kappa_{i, j-\frac{1}{2}, k}= \begin{cases}\kappa_{i, j-1, k}, & \text { se } \quad \hat{\kappa}_{i, j-1, k} \notin[0,1] \\ (2-\theta) \kappa_{i, j-1, k}-(1-\theta) \kappa_{i, j-2, k}, & \text { se } 0<\hat{\kappa}_{i, j-1, k}<a \\ \alpha_{D} \kappa_{i, j, k}+\alpha_{U} \kappa_{i, j-1, k}-\alpha_{R} \kappa_{i, j-2, k}, & \text { se } a \leqslant \hat{\kappa}_{i, j-1, k} \leqslant b \\ (1-\theta) \kappa_{i, j, k}+\theta \kappa_{i, j-1, k}, & \text { se } b<\hat{\kappa}_{i, j-1, k}<1\end{cases}
$$

na qual

$$
\mathbf{D}=(i, j, k), \quad \mathbf{U}=(i, j-1, k), \quad \mathbf{R}=(i, j-2, k) ;
$$

- Quando $\bar{v}_{2}=v_{i, j-\frac{1}{2}, k}<0$ e $\hat{\kappa}_{i, j, k}=\frac{\kappa_{i, j, k}-\kappa_{i, j+1, k}}{\kappa_{i, j-1, k}-\kappa_{i, j+1, k}}$ tem-se que

$$
\kappa_{i, j-\frac{1}{2}, k}= \begin{cases}\kappa_{i, j, k}, & \text { se } \hat{\kappa}_{i, j, k} \notin[0,1], \\ (2-\theta) \kappa_{i, j, k}-(1-\theta) \kappa_{i, j+1, k}, & \text { se } 0<\hat{\kappa}_{i, j, k}<a, \\ \alpha_{D} \kappa_{i, j-1, k}+\alpha_{U} \kappa_{i, j, k}-\alpha_{R} \kappa_{i, j+1, k}, & \text { se } a \leqslant \hat{\kappa}_{i, j, k} \leqslant b, \\ (1-\theta) \kappa_{i, j-1, k}+\theta \kappa_{i, j, k}, & \text { se } b<\hat{\kappa}_{i, j, k}<1,\end{cases}
$$

na qual

$$
\mathbf{D}=(i, j-1, k), \quad \mathbf{U}=(i, j, k), \quad \mathbf{R}=(i, j+1, k) ;
$$

(5) Aproximações para $\kappa_{i, j, k+\frac{1}{2}}$ : 
- Quando $\bar{w}_{1}=w_{i, j, k+\frac{1}{2}}>0$ e $\hat{\kappa}_{i, j, k}=\frac{\kappa_{i, j, k}-\kappa_{i, j, k-1}}{\kappa_{i, j, k+1}-\kappa_{i, j, k-1}}$ tem-se que

$$
\kappa_{i, j, k+\frac{1}{2}}= \begin{cases}\kappa_{i, j, k}, & \text { se } \hat{\kappa}_{i, j, k} \notin[0,1], \\ (2-\theta) \kappa_{i, j, k}-(1-\theta) \kappa_{i, j, k-1}, & \text { se } 0<\hat{\kappa}_{i, j, k}<a, \\ \alpha_{D} \kappa_{i, j, k+1}+\alpha_{U} \kappa_{i, j, k}-\alpha_{R} \kappa_{i, j, k-1}, & \text { se } a \leqslant \hat{\kappa}_{i, j, k} \leqslant b, \\ (1-\theta) \kappa_{i, j, k+1}+\theta \kappa_{i, j, k}, & \text { se } b<\hat{\kappa}_{i, j, k}<1,\end{cases}
$$

na qual

$$
\mathbf{D}=(i, j, k+1), \quad \mathbf{U}=(i, j, k), \quad \mathbf{R}=(i, j, k-1)
$$

- Quando $\bar{w}_{1}=w_{i, j, k+\frac{1}{2}}<0$ e $\hat{\kappa}_{i, j, k+1}=\frac{\kappa_{i, j, k+1}-\kappa_{i, j, k+2}}{\kappa_{i, j, k}-\kappa_{i, j, k+2}}$ tem-se que

$$
\kappa_{i, j, k+\frac{1}{2}}=\left\{\begin{array}{lll}
\kappa_{i, j, k+1}, & \text { se } & \hat{\kappa}_{i, j, k+1} \notin[0,1] \\
(2-\theta) \kappa_{i, j, k+1}-(1-\theta) \kappa_{i, j, k+2}, & \text { se } 0<\hat{\kappa}_{i, j, k+1}<a \\
\alpha_{D} \kappa_{i, j, k}+\alpha_{U} \kappa_{i, j, k+1}-\alpha_{R} \kappa_{i, j, k+2}, & \text { se } a \leqslant \hat{\kappa}_{i, j, k+1} \leqslant b \\
(1-\theta) \kappa_{i, j, k}+\theta \kappa_{i, j, k+2}, & \text { se } b<\hat{\kappa}_{i, j, k+1}<1
\end{array}\right.
$$

na qual

$$
\mathbf{D}=(i, j, k), \quad \mathbf{U}=(i, j, k+1), \quad \mathbf{R}=(i, j, k+2)
$$

(6) Aproximações para $\kappa_{i, j, k-\frac{1}{2}}$ :

- Quando $\bar{w}_{2}=w_{i, j, k-\frac{1}{2}}>0$ e $\hat{\kappa}_{i, j, k-1}=\frac{\kappa_{i, j, k-1}-\kappa_{i, j, k-2}}{\kappa_{i, j, k}-\kappa_{i, j, k-2}}$ tem-se que

$$
\kappa_{i, j, k-\frac{1}{2}}=\left\{\begin{array}{lll}
\kappa_{i, j, k-1}, & \text { se } & \hat{\kappa}_{i, j, k-1} \notin[0,1] \\
(2-\theta) \kappa_{i, j, k-1}-(1-\theta) \kappa_{i, j, k-2}, & \text { se } 0<\hat{\kappa}_{i, j, k-1}<a \\
\alpha_{D} \kappa_{i, j, k}+\alpha_{U} \kappa_{i, j, k-1}-\alpha_{R} \kappa_{i, j, k-2}, & \text { se } a \leqslant \hat{\kappa}_{i, j, k-1} \leqslant b \\
(1-\theta) \kappa_{i, j, k}+\theta \kappa_{i, j, k-1}, & \text { se } b<\hat{\kappa}_{i, j, k-1}<1
\end{array}\right.
$$

na qual

$$
\mathbf{D}=(i, j, k), \quad \mathbf{U}=(i, j, k-1), \quad \mathbf{R}=(i, j, k-2)
$$

- Quando $\bar{w}_{2}=w_{i, j, k-\frac{1}{2}}<0$ e $\hat{\kappa}_{i, j, k}=\frac{\kappa_{i, j, k}-\kappa_{i, j, k+1}}{\kappa_{i, j, k-1}-\kappa_{i, j, k+1}}$ tem-se que

$$
\kappa_{i, j, k-\frac{1}{2}}= \begin{cases}\kappa_{i, j, k}, & \text { se } \hat{\kappa}_{i, j, k} \notin[0,1], \\ (2-\theta) \kappa_{i, j, k}-(1-\theta) \kappa_{i, j, k+1}, & \text { se } 0<\hat{\kappa}_{i, j, k}<a, \\ \alpha_{D} \kappa_{i, j, k-1}+\alpha_{U} \kappa_{i, j, k}-\alpha_{R} \kappa_{i, j, k+1}, & \text { se } a \leqslant \hat{\kappa}_{i, j, k} \leqslant b, \\ (1-\theta) \kappa_{i, j, k-1}+\theta \kappa_{i, j, k}, & \text { se } b<\hat{\kappa}_{i, j, k}<1,\end{cases}
$$


na qual

$$
\mathbf{D}=(i, j, k-1), \quad \mathbf{U}=(i, j, k), \quad \mathbf{R}=(i, j, k+1)
$$

As outras derivadas do termo advectivo na equação de conservação (2.68) são determinadas e implementadas de maneira análoga.

É importante observar que, para aproximar as derivadas, o esquema ADBQUICKEST faz uso de três pontos vizinhos ( $\mathbf{D}, \mathbf{U}$ e $\mathbf{R})$. Dessa forma, podem ocorrer valores fora do domínio de solução para as células computacionais que estão próximas às fronteiras. Por exemplo, em (4.20), quando se aproxima $u_{i+\frac{1}{2}, j+\frac{1}{2}, k}$ para o caso de $\bar{v}_{1}<0$, o valor da propriedade transportada $u$ no ponto $\mathbf{R}=\left(i+\frac{1}{2}, j+2, k\right)$ deve estar definido. Analogamente, na aproximação (4.21), para aproximar $\kappa_{i-\frac{1}{2}, j, k}$ quando $\bar{u}_{2}>0$, o valor da propriedade transportada $\kappa$ no ponto $\mathbf{R}=$ $(i-2, j, k)$ é requerido. Em ambos os casos, o esquema híbrido (uma combinação do esquema "upwind" de primeira ordem e diferenças centradas) é utilizado.

Em resumo, as equações discretizadas e implementadas no ambiente de simulação Freeflow $3 D$ são como segue:

\section{- Continuidade}

A equação da continuidade (2.63) avaliada no ponto $(i, j, k)$ é dada por

$$
\frac{u_{i+\frac{1}{2}, j, k}-u_{i-\frac{1}{2}, j, k}}{\delta x}+\frac{v_{i, j+\frac{1}{2}, k}-v_{i, j-\frac{1}{2}, k}}{\delta y}+\frac{w_{i, j, k+\frac{1}{2}}-w_{i, j, k-\frac{1}{2}}}{\delta z}=0 .
$$

\section{- Quantidade de Movimento}

As equações (2.64), (2.65) e (2.66) avaliadas nos pontos $\left(i+\frac{1}{2}, j, k\right),\left(i, j+\frac{1}{2}, k\right)$ e $\left(i, j, k+\frac{1}{2}\right)$ respectivamente, são aproximadas por

$$
\begin{aligned}
\tilde{u}_{i+\frac{1}{2}, j, k}^{n+1}= & u_{i+\frac{1}{2}, j, k}^{n}+\delta t\left\{\left.\operatorname{CONV}(u)\right|_{i+\frac{1}{2}, j, k}-\frac{\tilde{p}_{e_{i+1, j, k}}-\tilde{p}_{e_{i, j, k}}}{\delta x}\right. \\
& +\frac{1}{\operatorname{Re} \delta y}\left[\left(\frac{u_{i+\frac{1}{2}, j+1, k}-u_{i+\frac{1}{2}, j, k}}{\delta y}-\frac{v_{i+1, j+\frac{1}{2}, k}-v_{i, j+\frac{1}{2}, k}}{\delta x}\right)\right. \\
& \left.-\left(\frac{u_{i+\frac{1}{2}, j, k}-u_{i+\frac{1}{2}, j-1, k}}{\delta y}-\frac{v_{i+1, j-\frac{1}{2}, k}-v_{i, j-\frac{1}{2}, k}}{\delta x}\right)\right] \\
& +\frac{1}{\operatorname{Re} \delta z}\left[\left(\frac{u_{i+\frac{1}{2}, j, k+1}-u_{i+\frac{1}{2}, j, k}}{\delta z}-\frac{w_{i+1, j, k+\frac{1}{2}}-w_{i, j, k+\frac{1}{2}}}{\delta x}\right)\right. \\
& \left.-\left(\frac{u_{i+\frac{1}{2}, j, k}-u_{i+\frac{1}{2}, j, k-1}}{\delta z}-\frac{w_{i+1, j, k-\frac{1}{2}}-w_{i, j, k-\frac{1}{2}}}{\delta x}\right)\right] \\
& +\alpha\left(\frac{2}{R e \delta x^{2}}\left[\nu_{t i+1, j, k}\left(u_{i+\frac{3}{2}, j, k}-u_{i+\frac{1}{2}, j, k}\right)-\nu_{t i, j, k}\left(u_{i+\frac{1}{2}, j, k}-u_{i-\frac{1}{2}, j, k}\right)\right]\right.
\end{aligned}
$$




$$
\begin{aligned}
& +\frac{1}{R e \delta y}\left[\nu_{t i+\frac{1}{2}, j+\frac{1}{2}, k}\left(\frac{u_{i+\frac{1}{2}, j+1, k}-u_{i+\frac{1}{2}, j, k}}{\delta y}+\frac{v_{i+1, j+\frac{1}{2}, k}-v_{i, j+\frac{1}{2}, k}}{\delta x}\right)\right. \\
& \left.-\nu_{t i+\frac{1}{2}, j-\frac{1}{2}, k}\left(\frac{u_{i+\frac{1}{2}, j, k}-u_{i+\frac{1}{2}, j-1, k}}{\delta y}+\frac{v_{i+1, j-\frac{1}{2}, k}-v_{i, j-\frac{1}{2}, k}}{\delta x}\right)\right] \\
& +\frac{1}{\operatorname{Re} \delta z}\left[\nu_{t i+\frac{1}{2}, j, k+\frac{1}{2}}\left(\frac{u_{i+\frac{1}{2}, j, k+1}-u_{i+\frac{1}{2}, j, k}}{\delta z}+\frac{w_{i+1, j, k+\frac{1}{2}}-w_{i, j, k+\frac{1}{2}}}{\delta x}\right)\right. \\
& \left.\left.\left.-\nu_{t i+\frac{1}{2}, j, k-\frac{1}{2}}\left(\frac{u_{i+\frac{1}{2}, j, k}-u_{i+\frac{1}{2}, j, k-1}}{\delta z}+\frac{w_{i+1, j, k-\frac{1}{2}}-w_{i, j, k-\frac{1}{2}}}{\delta x}\right)\right]\right)+\frac{1}{F r^{2}} g_{x}\right\}^{n} \text {, } \\
& \tilde{v}_{i, j+\frac{1}{2}, k}^{n+1}=v_{i, j+\frac{1}{2}, k}^{n}+\delta t\left\{\left.C O N V(v)\right|_{i, j+\frac{1}{2}, k}-\frac{\tilde{p}_{e_{i, j+1, k}}-\tilde{p}_{e_{i, j, k}}}{\delta y}\right. \\
& -\frac{1}{\operatorname{Re} \delta x}\left[\left(\frac{u_{i+\frac{1}{2}, j+1, k}-u_{i+\frac{1}{2}, j, k}}{\delta y}-\frac{v_{i+1, j+\frac{1}{2}, k}-v_{i, j+\frac{1}{2}, k}}{\delta x}\right)\right. \\
& \left.-\left(\frac{u_{i-\frac{1}{2}, j+1, k}-u_{i-\frac{1}{2}, j, k}}{\delta y}-\frac{v_{i, j+\frac{1}{2}, k}-v_{i-1, j+\frac{1}{2}, k}}{\delta x}\right)\right] \\
& +\frac{1}{\operatorname{Re} \delta z}\left[\left(\frac{v_{i, j+\frac{1}{2}, k+1}-v_{i, j+\frac{1}{2}, k}}{\delta z}-\frac{w_{i, j+1, k+\frac{1}{2}}-w_{i, j, k+\frac{1}{2}}}{\delta y}\right)\right. \\
& \left.-\left(\frac{v_{i, j+\frac{1}{2}, k}-v_{i, j+\frac{1}{2}, k-1}}{\delta z}-\frac{w_{i, j+1, k-\frac{1}{2}}-w_{i, j, k-\frac{1}{2}}}{\delta y}\right)\right] \\
& +\alpha\left(\frac{2}{\operatorname{Re} \delta y^{2}}\left(\nu_{t i, j+1, k}\left(v_{i, j+\frac{3}{2}, k}-v_{i, j+\frac{1}{2}, k}\right)-\nu_{t i, j, k}\left(v_{i, j+\frac{1}{2}, k}-v_{i, j-\frac{1}{2}, k}\right)\right)\right. \\
& +\frac{1}{\operatorname{Re} \delta x}\left[\nu_{t i+\frac{1}{2}, j+\frac{1}{2}, k}\left(\frac{u_{i+\frac{1}{2}, j+1, k}-u_{i+\frac{1}{2}, j, k}}{\delta y}+\frac{v_{i+1, j+\frac{1}{2}, k}-v_{i, j+\frac{1}{2}, k}}{\delta x}\right)\right. \\
& \left.-\nu_{t i-\frac{1}{2}, j+\frac{1}{2}, k}\left(\frac{u_{i-\frac{1}{2}, j+1, k}-u_{i-\frac{1}{2}, j, k}}{\delta y}+\frac{v_{i, j+\frac{1}{2}, k}-v_{i-1, j+\frac{1}{2}, k}}{\delta x}\right)\right] \text {. } \\
& +\frac{1}{\operatorname{Re} \delta z}\left[\nu_{t i, j+\frac{1}{2}, k+\frac{1}{2}}\left(\frac{v_{i, j+\frac{1}{2}, k+1}-v_{i, j+\frac{1}{2}, k}}{\delta z}+\frac{w_{i, j+1, k+\frac{1}{2}}-w_{i, j, k+\frac{1}{2}}}{\delta y}\right)\right. \\
& \left.\left.\left.-\nu_{t i, j+\frac{1}{2}, k-\frac{1}{2}}\left(\frac{v_{i, j+\frac{1}{2}, k}-v_{i, j+\frac{1}{2}, k-1}}{\delta z}+\frac{w_{i, j+1, k-\frac{1}{2}}-w_{i, j, k-\frac{1}{2}}}{\delta y}\right)\right]\right)+\frac{1}{F r^{2}} g_{y}\right\}^{n} \text {, }
\end{aligned}
$$




$$
\begin{aligned}
\tilde{w}_{i, j, k+\frac{1}{2}}^{n+1}= & w_{i, j, k+\frac{1}{2}}^{n}+\delta t\left\{\left.C O N V(w)\right|_{i, j, k+\frac{1}{2}}-\frac{\tilde{p}_{e_{i, j, k+1}}-\tilde{p}_{e_{i, j, k}}}{\delta z}\right. \\
& -\frac{1}{R e \delta x}\left[\left(\frac{u_{i+\frac{1}{2}, j, k+1}-u_{i+\frac{1}{2}, j, k}}{\delta z}-\frac{w_{i+1, j, k+\frac{1}{2}}-w_{i, j, k+\frac{1}{2}}}{\delta x}\right)\right. \\
& \left.-\left(\frac{u_{i-\frac{1}{2}, j, k+1}-u_{i-\frac{1}{2}, j, k}}{\delta z}-\frac{w_{i, j, k+\frac{1}{2}}-w_{i-1, j, k+\frac{1}{2}}}{\delta x}\right)\right] \\
& -\frac{1}{\operatorname{Re} \delta y}\left[\left(\frac{v_{i, j+\frac{1}{2}, k+1}-v_{i, j+\frac{1}{2}, k}}{\delta z}-\frac{w_{i, j+1, k+\frac{1}{2}}-w_{i, j, k+\frac{1}{2}}}{\delta y}\right)\right. \\
& -\left(\frac{\left.\left.v_{i, j-\frac{1}{2}, k+1}-v_{i, j-\frac{1}{2}, k}-\frac{w_{i, j, k+\frac{1}{2}}-w_{i, j-1, k+\frac{1}{2}}}{\delta y}\right)\right]}{\delta z}\right) \\
& +\alpha\left(\frac{2}{R e \delta z^{2}}\left(\nu_{t i, j, k+1}\left(w_{i, j, k+\frac{3}{2}}-w_{i, j, k+\frac{1}{2}}\right)-\nu_{t i, j, k}\left(w_{i, j, k+\frac{1}{2}}-w_{i, j, k-\frac{1}{2}}\right)\right)\right. \\
& +\frac{1}{R e \delta x}\left[\nu_{t i+\frac{1}{2}, j, k+\frac{1}{2}}\left(\frac{u_{i+\frac{1}{2}, j, k+1}-u_{i+\frac{1}{2}, j, k}}{\delta z}+\frac{w_{i+1, j, k+\frac{1}{2}}-w_{i, j, k+\frac{1}{2}}}{\delta x}\right)\right. \\
& -\nu_{t i-\frac{1}{2}, j, k+\frac{1}{2}}\left(\frac{\left.\left.u_{i-\frac{1}{2}, j, k+1}-u_{i-\frac{1}{2}, j, k}+\frac{w_{i, j, k+\frac{1}{2}}-w_{i-1, j, k+\frac{1}{2}}}{\delta z}\right)\right] .}{\delta x}\right) \\
& +\frac{1}{R e \delta y}\left[\nu_{t i, j+\frac{1}{2}, k+\frac{1}{2}}\left(\frac{v_{i, j+\frac{1}{2}, k+1}-v_{i, j+\frac{1}{2}, k}+\frac{w_{i, j+1, k+\frac{1}{2}}-w_{i, j, k+\frac{1}{2}}}{\delta y}}{\delta z}\right)\right. \\
& \left.\left.-\nu_{t i, j-\frac{1}{2}, k+\frac{1}{2}}\left(\frac{v_{i, j+\frac{1}{2}, k+1}-v_{i, j+\frac{1}{2}, k}}{\delta z}+\frac{w_{i, j, k+\frac{1}{2}}-w_{i, j-1, k+\frac{1}{2}}}{\delta y}\right)\right]+\frac{1}{F r^{2}} g_{z}\right\}^{n} .
\end{aligned}
$$

\section{- Equação de Poisson para $\psi$}

A equação de Poisson dada por (4.6) pode ser escrita como

$$
\frac{\partial^{2} \psi}{\partial x^{2}}+\frac{\partial^{2} \psi}{\partial y^{2}}+\frac{\partial^{2} \psi}{\partial z^{2}}=\frac{\partial \tilde{u}}{\partial x}+\frac{\partial \tilde{v}}{\partial y}+\frac{\partial \tilde{w}}{\partial z}
$$

Aproximando-se as derivadas por diferenças centrais obtêm-se

$$
\begin{aligned}
\tilde{D}_{i, j, k}= & \frac{\psi_{i+1, j, k}-2 \psi_{i, j, k}+\psi_{i-1, j, k}}{\delta x^{2}}+\frac{\psi_{i, j+1, k}-2 \psi_{i, j, k}+\psi_{i, j-1, k}}{\delta y^{2}} \\
& +\frac{\psi_{i, j, k+1}-2 \psi_{i, j, k}+\psi_{i, j, k-1}}{\delta z^{2}}
\end{aligned}
$$

em que

$$
\tilde{D}_{i, j, k}=\frac{\tilde{u}_{i+\frac{1}{2}, j, k}-\tilde{u}_{i-\frac{1}{2}, j, k}}{\delta x}+\frac{\tilde{v}_{i, j+\frac{1}{2}, k}-\tilde{v}_{i, j-\frac{1}{2}, k}}{\delta y}+\frac{\tilde{w}_{i, j, k+\frac{1}{2}}-\tilde{v}_{i, j, k-\frac{1}{2}}}{\delta z} .
$$

A equação (4.22) quando aplicada em todos os pontos do domínio gera um sistema de equações lineares, cuja matriz dos coeficientes é simétrica, definida positiva e esparsa, e os termos independentes é o vetor divergente $\tilde{D}_{i, j, k}$. 


\section{- Energia Cinética}

A equação (2.67) avaliada no ponto $(i, j, k)$ é aproximada por

$$
\begin{aligned}
\kappa_{i, j, k}^{n+1}= & \kappa_{i, j, k}^{n}+\delta t\left\{\left.\operatorname{CONV}(\kappa)\right|_{i, j, k}\right. \\
& +\frac{1}{R e}\left[\frac { 1 } { \delta x ^ { 2 } } \left(\left(1+\frac{\nu_{t i+1, j, k}+\nu_{t i, j, k}}{2 \sigma_{\kappa}}\right)\left(\kappa_{i+1, j, k}-\kappa_{i, j, k}\right)\right.\right. \\
& \left.-\left(1+\frac{\nu_{t i, j, k}+\nu_{t i-1, j, k}}{2 \sigma_{\kappa}}\right)\left(\kappa_{i, j, k}-\kappa_{i-1, j, k}\right)\right) \\
& +\frac{1}{\delta y^{2}}\left(\left(1+\frac{\nu_{t i, j+1, k}+\nu_{t i, j, k}}{2 \sigma_{\kappa}}\right)\left(\kappa_{i, j+1, k}-\kappa_{i, j, k}\right)\right. \\
& \left.-\left(1+\frac{\nu_{t i, j, k}+\nu_{t i, j-1, k}}{2 \sigma_{\kappa}}\right)\left(\kappa_{i, j, k}-\kappa_{i, j-1, k}\right)\right) \\
& +\frac{1}{\delta z^{2}}\left(\left(1+\frac{\nu_{t i, j, k+1}+\nu_{t i, j, k}}{2 \sigma_{\kappa}}\right)\left(\kappa_{i, j, k+1}-\kappa_{i, j, k}\right)\right. \\
& \left.\left.\left.-\left(1+\frac{\nu_{t i, j, k}+\nu_{t i, j, k-1}}{2 \sigma_{\kappa}}\right)\left(\kappa_{i, j, k}-\kappa_{i, j, k-1}\right)\right)\right]+P_{i, j, k}-\varepsilon_{i, j, k}\right\}^{n} .
\end{aligned}
$$

\section{- Dissipação de Energia}

A equação (2.68) avaliada no ponto $(i, j, k)$ é aproximada por

$$
\begin{aligned}
\varepsilon_{i, j, k}^{n+1}= & \varepsilon_{i, j, k}^{n}+\delta t\left\{\left.\operatorname{CONV}(\varepsilon)\right|_{i, j, k}\right. \\
& +\frac{1}{R e}\left[\frac { 1 } { \delta x ^ { 2 } } \left(\left(1+\frac{\nu_{t i+1, j, k}+\nu_{t i, j, k}}{2 \sigma_{\varepsilon}}\right)\left(\varepsilon_{i+1, j, k}-\varepsilon_{i, j, k}\right)\right.\right. \\
& \left.-\left(1+\frac{\nu_{t i, j, k}+\nu_{t i-1, j, k}}{2 \sigma_{\varepsilon}}\right)\left(\varepsilon_{i, j, k}-\varepsilon_{i-1, j, k}\right)\right) \\
& +\frac{1}{\delta y^{2}}\left(\left(1+\frac{\nu_{t i, j+1, k}+\nu_{t i, j, k}}{2 \sigma_{\varepsilon}}\right)\left(\varepsilon_{i, j+1, k}-\varepsilon_{i, j, k}\right)\right. \\
& \left.-\left(1+\frac{\nu_{t i, j, k}+\nu_{t i, j-1, k}}{2 \sigma_{\varepsilon}}\right)\left(\varepsilon_{i, j, k}-\varepsilon_{i, j-1, k}\right)\right) \\
& +\frac{1}{\delta z^{2}}\left(\left(1+\frac{\nu_{t i, j, k+1}+\nu_{t i, j, k}}{2 \sigma_{\varepsilon}}\right)\left(\varepsilon_{i, j, k+1}-\varepsilon_{i, j, k}\right)\right. \\
& \left.\left.-\left(1+\frac{\nu_{t i, j, k}+\nu_{t i, j, k-1}}{2 \sigma_{\varepsilon}}\right)\left(\varepsilon_{i, j, k}-\varepsilon_{i, j, k-1}\right)\right)\right] \\
& \left.+\left(C_{1 \varepsilon} P_{i, j, k}-C_{2 \varepsilon} \varepsilon_{i, j, k}\right) / T_{i, j, k}\right\}^{n}
\end{aligned}
$$




\section{- Produção de Turbulência}

A equação (2.56) avaliada no ponto $(i, j, k)$ é aproximada por

$$
\begin{aligned}
P_{i, j, k}^{n}=\nu_{t i, j, k}^{n}\left\{\left[\frac{2}{\delta x^{2}}\left(u_{i+\frac{1}{2}, j, k}-u_{i-\frac{1}{2}, j, k}\right)^{2}\right.\right. \\
\left.+\frac{2}{\delta y^{2}}\left(v_{i, j+\frac{1}{2}, k}-v_{i, j-\frac{1}{2}, k}\right)^{2}+\frac{2}{\delta z^{2}}\left(w_{i, j, k+\frac{1}{2}}-w_{i, j, k-\frac{1}{2}}\right)^{2}\right] \\
+\left[\frac{1}{4 \delta y}\left(u_{i+\frac{1}{2}, j+1, k}+u_{i-\frac{1}{2}, j+1, k}-u_{i+\frac{1}{2}, j-1, k}-u_{i-\frac{1}{2}, j-1, k}\right)\right. \\
\left.+\frac{1}{4 \delta x}\left(v_{i+1, j+\frac{1}{2}, k}+v_{i+1, j-\frac{1}{2}, k}-v_{i-1, j+\frac{1}{2}, k}-v_{i-1, j-\frac{1}{2}, k}\right)\right]^{2} \\
+\left[\frac{1}{4 \delta z}\left(u_{i+1 / 2, j, k+1}+u_{i-1 / 2, j, k+1}-u_{i+1 / 2, j, k-1}-u_{i-1 / 2, j, k-1}\right)\right. \\
\left.+\frac{1}{4 \delta x}\left(w_{i+1, j, k+1 / 2}+w_{i+1, j, k-1 / 2}-w_{i-1, j, k+1 / 2}-w_{i-1, j, k-1 / 2}\right)\right]^{2} \\
+\left[\frac{1}{4 \delta z}\left(v_{i, j+1 / 2, k+1}+v_{i, j-1 / 2, k+1}-v_{i, j+1 / 2, k-1}-v_{i, j-1 / 2, k-1}\right)\right. \\
\left.\left.+\frac{1}{4 \delta y}\left(w_{i, j+1, k+1 / 2}+w_{i, j+1, k-1 / 2}-w_{i, j-1, k+1 / 2}-w_{i, j-1, k-1 / 2}\right)\right]^{2}\right\}^{n} .
\end{aligned}
$$

\section{- Velocidade Final}

Considera-se a equação (4.5) que pode ser escrita como

$$
\begin{aligned}
& u^{n+1}=\tilde{u}^{n+1}-\frac{\partial \psi^{n+1}}{\partial x}, \\
& v^{n+1}=\tilde{v}^{n+1}-\frac{\partial \psi^{n+1}}{\partial y}, \\
& w^{n+1}=\tilde{w}^{n+1}-\frac{\partial \psi^{n+1}}{\partial z} .
\end{aligned}
$$

Avaliando-se as três expressões anteriores nos pontos $\left(i+\frac{1}{2}, j, k\right),\left(i, j+\frac{1}{2}, k\right)$ e $\left(i, j, k+\frac{1}{2}\right)$, respectivamente, obtêm-se

$$
\begin{aligned}
& u_{i+\frac{1}{2}, j, k}^{n+1}=\tilde{u}_{i+\frac{1}{2}, j, k}^{n+1}-\frac{\psi_{i+1, j, k}^{n+1}-\psi_{i, j, k}^{n+1}}{\delta x}, \\
& v_{i, j+\frac{1}{2}, k}^{n+1}=\tilde{v}_{i, j+\frac{1}{2}, k}^{n+1}-\frac{\psi_{i, j+1, k}^{n+1}-\psi_{i, j, k}^{n+1}}{\delta y}, \\
& w_{i, j, k+\frac{1}{2}}^{n+1}=\tilde{w}_{i, j, k+\frac{1}{2}}^{n+1}-\frac{\psi_{i, j, k+1}^{n+1}-\psi_{i, j, k}^{n+1}}{\delta z} .
\end{aligned}
$$

\section{- Pressão}


A equação (4.8) avaliada no ponto $(i, j, k)$ é aproximada por

$$
p_{e_{i, j, k}^{n+1}}^{n+1} \tilde{p}_{e_{i, j, k}}+\frac{\psi_{i, j, k}^{n+1}}{\delta t}
$$

\subsubsection{Discretizações das condições de contorno}

Nas implementações, as condições de contorno nas superfícies livre e rígida são aproximadas localmente por diferenças finitas utilizando-se as mesmas idéias descritas em Tomé et al. [140] (caso 3D).

As condições de contorno aplicadas na superfície livre do fluido dadas pelas equações (2.97)-(2.99) (caso laminar) e (2.101)-(2.103) (caso turbulento) são descritas na sequência. No caso laminar essas condições estão bem explicadas em Tomé et al. [140] e Oishi [102], e para o caso turbulento essas condições são discutidas agora. Para tanto, assume-se que a malha computacional é suficientemente fina de modo que esta superfície livre seja aproximada por superfícies planares. As condições (2.101), (2.102) e (2.103) são, então, aplicadas para os seguintes casos, a saber:

(a) Superfícies 1D: Essas superfícies são definidas por planos perpendiculares a um dos eixos coordenados e são identificadas por células (S) que tem apenas uma face em contato com a célula (E). Neste caso, assume-se que o vetor normal aponta para a direção de um dos eixos coordenados e tome uma das formas $\mathbf{n}=( \pm 1,0,0), \mathbf{n}=(0, \pm 1,0)$ ou $\mathbf{n}=(0,0, \pm 1)$.

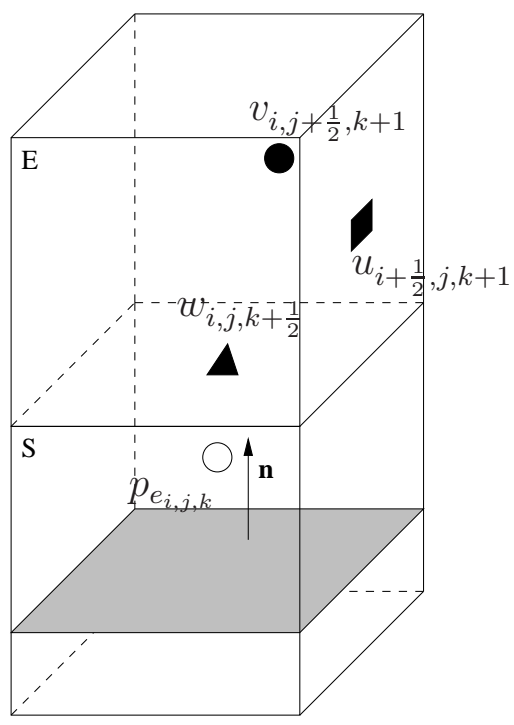

Figura 4.3: Exemplo de uma superfície planar paralela ao plano formado pelos eixos $x y$ com $\mathbf{n}=(0,0,1)$.

A Figura 4.3 ilustra um exemplo para o caso particular de uma célula $(\mathbf{S})$ com a face $\left(k+\frac{1}{2}\right)$ em contato com uma célula $(\mathbf{E})$. Neste caso tem-se $\mathbf{n}=(0,0,1), \mathbf{m}_{\mathbf{1}}=(0,1,0)$ e $\mathbf{m}_{\mathbf{2}}=(1,0,0)$. 
Logo, as equações (2.101), (2.102) e (2.103) são reduzidas, respectivamente, a

$$
\begin{gathered}
p_{e}=\frac{2}{R e}\left(1+\nu_{t}\right)\left(\frac{\partial w}{\partial z}\right) \\
\frac{1}{R e}\left(1+\nu_{t}\right)\left[\left(\frac{\partial v}{\partial z}+\frac{\partial w}{\partial y}\right)\right]=0
\end{gathered}
$$

e

$$
\frac{1}{R e}\left(1+\nu_{t}\right)\left[\left(\frac{\partial u}{\partial z}+\frac{\partial w}{\partial x}\right)\right]=0
$$

Observa-se que para o cálculo das velocidades intermediárias usando as equações de quantidade de movimento (2.64), (2.65) e (2.66), os valores $u_{i+\frac{1}{2}, j, k+1}, v_{i, j+\frac{1}{2}, k+1}, w_{i, j, k+\frac{1}{2}}$ e $p_{e_{i, j, k}}$ devem estar disponíveis (ver Figura 4.3). Para tanto, as equações (4.23), (4.24) e (4.25) são avaliadas nos pontos $(i, j, k),\left(i, j+\frac{1}{2}, k+\frac{1}{2}\right)$ e $\left(i+\frac{1}{2}, j, k+\frac{1}{2}\right)$, respectivamente, e aproximadas por diferenças finitas. Em suma, esses valores são obtidos como segue.

Em primeiro lugar, discretiza-se a equação da continuidade $(2.63)$ no ponto $(i, j, k)$ para obter

$$
\frac{u_{i+\frac{1}{2}, j, k}-u_{i-\frac{1}{2}, j, k}}{\delta x}+\frac{v_{i, j+\frac{1}{2}, k}-v_{i, j-\frac{1}{2}, k}}{\delta y}+\frac{w_{i, j, k+\frac{1}{2}}-w_{i, j, k-\frac{1}{2}}}{\delta z}=0,
$$

donde obtém-se

$$
w_{i, j, k+\frac{1}{2}}=w_{i, j, k-\frac{1}{2}}-\frac{\delta z}{\delta x}\left(u_{i+\frac{1}{2}, j, k}-u_{i-\frac{1}{2}, j, k}\right)-\frac{\delta z}{\delta y}\left(v_{i, j+\frac{1}{2}, k}-v_{i, j-\frac{1}{2}, k}\right) .
$$

Em seguida, discretiza-se (4.25) no ponto $\left(i+\frac{1}{2}, j, k+\frac{1}{2}\right)$ para obter

$$
\frac{1}{R e}\left(1+\nu_{t_{i}+\frac{1}{2}, j, k+\frac{1}{2}}\right)\left(\frac{u_{i+\frac{1}{2}, j, k+1}-u_{i+\frac{1}{2}, j, k}}{\delta z}+\frac{w_{i+1, j, k+\frac{1}{2}}-w_{i, j, k+\frac{1}{2}}}{\delta x}\right)=0,
$$

na qual, isolando-se o termo $u_{i+\frac{1}{2}, j, k}$, vem

$$
u_{i+\frac{1}{2}, j, k+1}=u_{i+\frac{1}{2}, j, k}-\frac{\delta z}{\delta x}\left(w_{i+1, j, k+\frac{1}{2}}-w_{i, j, k+\frac{1}{2}}\right)
$$

Da mesma forma, discretiza-se (4.24) no ponto $\left(i, j+\frac{1}{2}, k+\frac{1}{2}\right)$ para obter

$$
\frac{1}{R e}\left(1+\nu_{t_{i}, j+\frac{1}{2}, k+\frac{1}{2}}\right)\left(\frac{v_{i, j+\frac{1}{2}, k+1}-v_{i, j+\frac{1}{2}, k}}{\delta z}+\frac{w_{i, j+1, k+\frac{1}{2}}-w_{i, j, k+\frac{1}{2}}}{\delta y}\right)=0,
$$

em que, o termo $v_{i, j+\frac{1}{2}, k+1}$ é calculado por

$$
v_{i, j+\frac{1}{2}, k+1}=v_{i, j+\frac{1}{2}, k}-\frac{\delta z}{\delta y}\left(w_{i, j+1, k+\frac{1}{2}}-w_{i, j, k+\frac{1}{2}}\right) .
$$


Por fim, uma vez obtidas as velocidades $u_{i+\frac{1}{2}, j, k+1}, v_{i, j+\frac{1}{2}, k+1}, w_{i, j, k+\frac{1}{2}}$, a pressão $p_{e_{i, j, k}}$ é calculada por meio da equação (4.23) avaliada no ponto $(i, j, k)$, cujo resultado é dado por

$$
p_{e_{i, j, k}}=\frac{2}{R e}\left(1+\nu_{t i, j, k}\right)\left(\frac{w_{i, j, k+\frac{1}{2}}-w_{i, j, k-\frac{1}{2}}}{\delta z}\right)
$$

Os outros casos para esse tipo de superfícies com $\mathbf{n}=(0, \pm 1,0)$ e $\mathbf{n}=( \pm 1,0,0)$ são tratados de forma análoga.

(b) Superfícies 2D: São superfície definidas por planos inclinados de $45^{\circ}$ e são identificadas por células (S) que possuem duas faces adjacente em contato com faces de células (E). Nessas superfícies, admite-se que o vetor normal unitário pode ser tomado como $\mathbf{n}=\left( \pm \frac{\sqrt{2}}{2}, \pm \frac{\sqrt{2}}{2}, 0\right)$, $\mathbf{n}=\left( \pm \frac{\sqrt{2}}{2}, 0, \pm \frac{\sqrt{2}}{2}\right)$ ou $\mathbf{n}=\left(0, \pm \frac{\sqrt{2}}{2}, \pm \frac{\sqrt{2}}{2}\right)$.

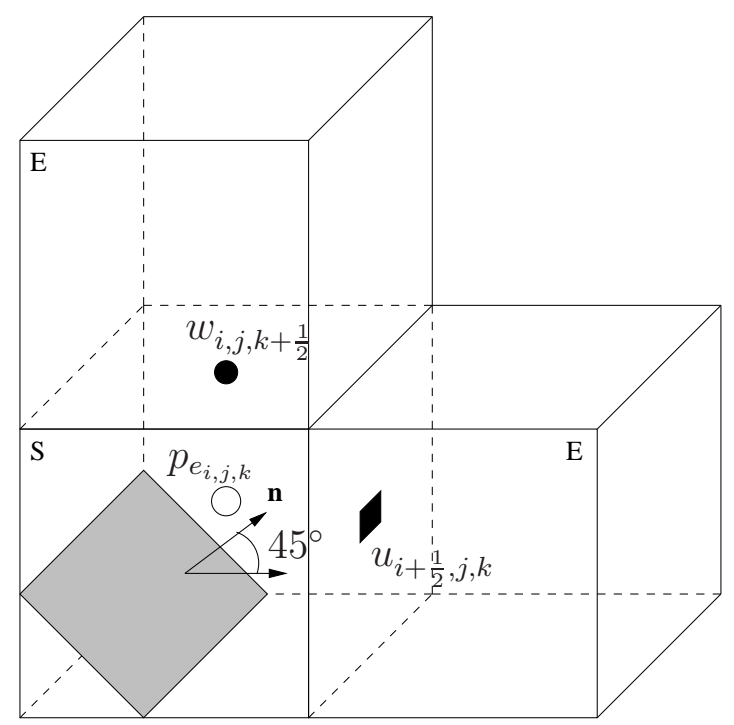

Figura 4.4: Exemplo de uma superfície planar inclinada de $45^{\circ} \operatorname{com} \mathbf{n}=\left(\frac{\sqrt{2}}{2}, 0, \frac{\sqrt{2}}{2}\right)$.

A Figura 4.4 ilustra o caso de uma célula $(\mathbf{S})$ com as faces $\left(k+\frac{1}{2}\right)$ e $\left(i+\frac{1}{2}\right)$ em contato com uma célula $(\mathbf{E})$. Neste caso, admitem-se que o vetor normal unitário é dado por $\mathbf{n}=\left(\frac{\sqrt{2}}{2}, 0, \frac{\sqrt{2}}{2}\right)$ e os vetores tangenciais por $\mathbf{m}_{1}=\left(\frac{\sqrt{2}}{2}, 0,-\frac{\sqrt{2}}{2}\right)$ e $\mathbf{m}_{\mathbf{2}}=(0,1,0)$. Logo, as equações $(2.101)$, (2.102) e (2.103) reduzem-se a

$$
p_{e}=\frac{2}{R e}\left(1+\nu_{t}\right)\left(\frac{\partial u}{\partial x}+\frac{\partial w}{\partial z}+\frac{\partial u}{\partial z}+\frac{\partial w}{\partial x}\right)
$$

$\mathrm{e}$

$$
\frac{1}{R e}\left(1+\nu_{t}\right)\left[\left(\frac{\partial u}{\partial x}-\frac{\partial w}{\partial z}\right)\right]=0
$$

Como pode-se observar na Figura 4.4, as velocidades $u_{i+\frac{1}{2}, j, k}$ e $w_{i, j, k+\frac{1}{2}}$ são requeridas e 
devem ser calculadas aplicando-se a equação da continuidade (2.42) e a equação (4.31) no ponto $(i, j, k)$. Dessa maneira, tem-se

$$
\frac{u_{i+\frac{1}{2}, j, k}-u_{i-\frac{1}{2}, j, k}}{\delta x}+\frac{w_{i, j, k+\frac{1}{2}}-w_{i, j, k-\frac{1}{2}}}{\delta z}=-\left(\frac{v_{i, j+\frac{1}{2}, k}-v_{i, j-\frac{1}{2}, k}}{\delta y}\right)
$$

e

$$
\frac{1}{R e}\left(1+\nu_{t_{i, j, k}}\right)\left(\frac{u_{i+\frac{1}{2}, j, k}-u_{i-\frac{1}{2}, j, k}}{\delta x}-\frac{w_{i, j, k+\frac{1}{2}}-w_{i, j, k-\frac{1}{2}}}{\delta z}\right)=0 .
$$

Resolvendo (4.32) e (4.33) para $u_{i+\frac{1}{2}, j, k}$ e $w_{i, j, k+\frac{1}{2}}$ obtém-se

$$
u_{i+\frac{1}{2}, j, k}=u_{i-\frac{1}{2}, j, k}-\frac{1}{2} \frac{\delta x}{\delta y}\left(v_{i, j+\frac{1}{2}, k}-v_{i, j-\frac{1}{2}, k}\right)
$$

e

$$
w_{i, j, k+\frac{1}{2}}=w_{i, j, k-\frac{1}{2}}-\frac{1}{2} \frac{\delta z}{\delta y}\left(v_{i, j+\frac{1}{2}, k}-v_{i, j-\frac{1}{2}, k}\right)
$$

Calculado os valores de $u_{i+\frac{1}{2}, j, k}$ e $w_{i, j, k+\frac{1}{2}}$, a pressão é obtida por meio da equação (4.30) aplicada no centro da célula, isto é,

$$
\begin{aligned}
p_{e_{i, j, k}}= & \frac{1}{R e}\left(1+\nu_{t i, j, k}\right)\left[\frac{u_{i+\frac{1}{2}, j, k}-u_{i-\frac{1}{2}, j, k}}{\delta x}+\frac{w_{i, j, k+\frac{1}{2}}-w_{i, j, k-\frac{1}{2}}}{\delta z}\right. \\
& +\frac{1}{2}\left(\frac{u_{i+\frac{1}{2}, j, k}+u_{i-\frac{1}{2}, j, k}-u_{i+\frac{1}{2}, j, k-1}-u_{i-\frac{1}{2}, j, k-1}}{\delta z}\right. \\
& \left.\left.+\frac{w_{i, j, k+\frac{1}{2}}+w_{i, j, k-\frac{1}{2}}-w_{i-1, j, k+\frac{1}{2}}-w_{i-1, j, k-\frac{1}{2}}}{\delta x}\right)\right] .
\end{aligned}
$$

As equações correspondentes para os outros casos são obtidas de forma semelhante.

(c) Superfícies 3D: Essas superfícies são definidas por planos inclinada de $60^{\circ}$ e são identificadas por células (S) que possuem três faces adjacentes em contato com faces de células (E). Nestas configurações, assume-se o vetor normal unitário faz um ângulo de $60^{\circ}$ com os eixos coordenados e tem a forma $\mathbf{n}=\left( \pm \frac{\sqrt{3}}{3}, \pm \frac{\sqrt{3}}{3}, \pm \frac{\sqrt{3}}{3}\right)$.

A Figura 4.5 ilustra o caso particular de uma célula $(\mathbf{S})$ com as faces $\left(i+\frac{1}{2}\right),\left(j-\frac{1}{2}\right)$ e $\left(k+\frac{1}{2}\right)$ em contato com as faces de células $(\mathbf{E})$. Neste caso, admite-se que o vetor normal é da forma $\mathbf{n}=\left(\frac{\sqrt{3}}{3},-\frac{\sqrt{3}}{3}, \frac{\sqrt{3}}{3}\right)$ e os vetores tangencias dados por $\mathbf{m}_{\mathbf{1}}=\left(\frac{\sqrt{2}}{2}, 0,-\frac{\sqrt{2}}{2}\right)$ e $\mathbf{m}_{\mathbf{2}}=$ $\left(\frac{\sqrt{6}}{6}, \frac{2 \sqrt{6}}{6}, \frac{\sqrt{6}}{6}\right)$. Com estas configurações, as equações (2.101), (2.102) e (2.103) reduzem-se a

$$
p_{e}-\frac{2}{3 R e}\left(1+\nu_{t}\right)\left[-\left(\frac{\partial u}{\partial y}+\frac{\partial v}{\partial x}\right)+\left(\frac{\partial u}{\partial z}+\frac{\partial w}{\partial x}\right)-\left(\frac{\partial v}{\partial z}+\frac{\partial w}{\partial y}\right)\right]=0
$$




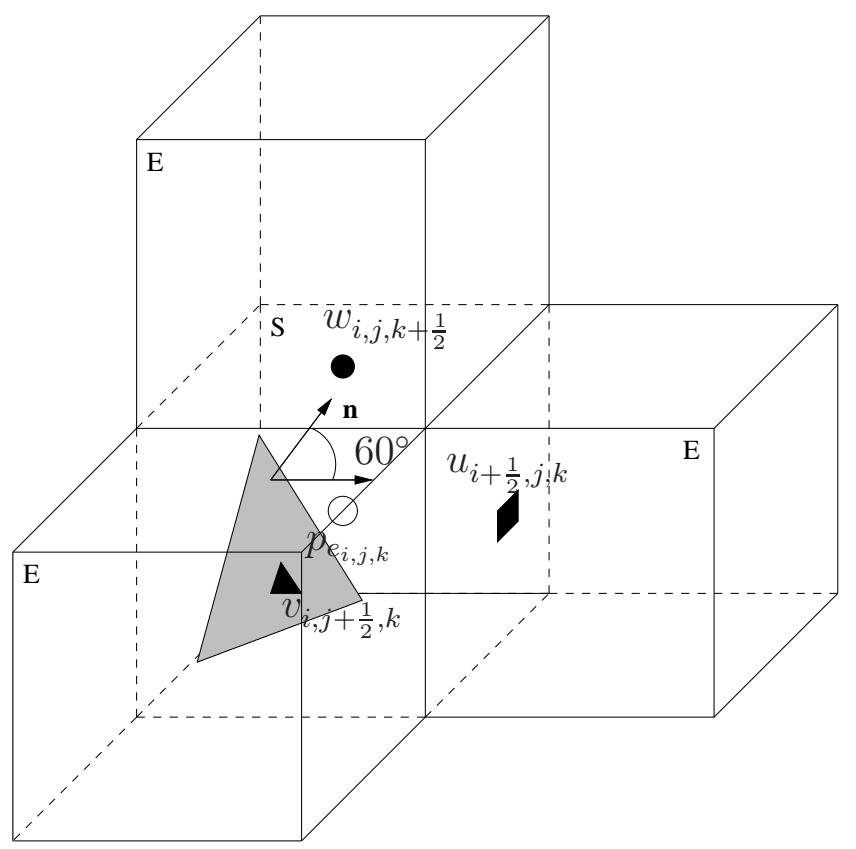

Figura 4.5: Exemplo de uma superfície planar inclinada de $60^{\circ} \operatorname{com} \mathbf{n}=\left(\frac{\sqrt{3}}{3},-\frac{\sqrt{3}}{3}, \frac{\sqrt{3}}{3}\right)$.

$$
\frac{1}{R e}\left(1+\nu_{t}\right)\left[2 \frac{\partial u}{\partial x}-2 \frac{\partial w}{\partial z}-\left(\frac{\partial u}{\partial y}+\frac{\partial v}{\partial x}\right)+\left(\frac{\partial v}{\partial z}+\frac{\partial w}{\partial y}\right)\right]=0
$$

e

$$
\frac{1}{R e}\left(1+\nu_{t}\right)\left[2 \frac{\partial u}{\partial x}-4 \frac{\partial v}{\partial y}+2 \frac{\partial w}{\partial z}+\left(\frac{\partial u}{\partial y}+\frac{\partial v}{\partial x}\right)+2\left(\frac{\partial u}{\partial z}+\frac{\partial w}{\partial x}\right)+\left(\frac{\partial v}{\partial z}+\frac{\partial w}{\partial y}\right)\right]=0 .
$$

Observa-se na Figura 4.5 que é necessário determinar as velocidades $u_{i+\frac{1}{2}, j, k}, v_{i, j+\frac{1}{2}, k}$ e $w_{i, j, k+\frac{1}{2}}$ de modo que a equação da continuidade seja utilizada. Assim, somando-se as equações (4.38) e (4.39) obtém-se

$$
\frac{1}{R e}\left(1+\nu_{t}\right)\left[4 \frac{\partial u}{\partial x}-4 \frac{\partial v}{\partial y}+2\left(\frac{\partial u}{\partial z}+\frac{\partial w}{\partial x}\right)+2\left(\frac{\partial v}{\partial z}+\frac{\partial w}{\partial y}\right)\right]=0
$$

Para obter os valores de $u_{i+\frac{1}{2}, j, k}, v_{i, j+\frac{1}{2}, k}$ e $w_{i, j, k+\frac{1}{2}}$ é necessário resolver um sistema linear $3 \times 3$ obtido pelas discretizações das equações (4.38), (4.39), (4.40) e da equação da continuidade (2.42) aplicadas no ponto $(i, j, k)$ da célula $(\mathbf{S})$. Esse sistema é então resolvido pelo método de eliminação de Gauss. Mais detalhes sobre a construção desse sistema linear podem ser encontrados em Tomé et al. [140]. Obtidas as velocidades $u_{i+\frac{1}{2}, j, k}, v_{i, j+\frac{1}{2}, k}$ e $w_{i, j, k+\frac{1}{2}}$, a pressão 
é calculada no centro da célula através da equação (4.37), isto é,

$$
\begin{aligned}
p_{e_{i, j, k}}= & \frac{2}{3 R e}\left(1+\nu_{t i, j, k}\right)\left[-\left(\frac{u_{i+\frac{1}{2}, j+1, k}+u_{i-\frac{1}{2}, j+1, k}-u_{i+\frac{1}{2}, j, k}-u_{i-\frac{1}{2}, j, k}}{\delta y}\right.\right. \\
& \left.+\frac{v_{i, j+\frac{1}{2}, k}+v_{i, j-\frac{1}{2}, k}-v_{i-1, j+\frac{1}{2}, k}-v_{i-1, j-\frac{1}{2}, k}}{\delta x}\right) \\
& +\left(\frac{u_{i+\frac{1}{2}, j, k}+u_{i-\frac{1}{2}, j, k}-u_{i+\frac{1}{2}, j, k-1}-u_{i-\frac{1}{2}, j, k-1}}{\delta z}\right. \\
& \left.+\frac{w_{i, j, k+\frac{1}{2}}+w_{i, j, k-\frac{1}{2}}-w_{i-1, j, k+\frac{1}{2}}-w_{i-1, j, k-\frac{1}{2}}}{\delta x}\right) \\
& -\left(\frac{v_{i, j+\frac{1}{2}, k}+v_{i, j-\frac{1}{2}, k}-v_{i, j+\frac{1}{2}, k-1}-v_{i, j-\frac{1}{2}, k-1}}{\delta z}\right. \\
& \left.\left.+\frac{w_{i, j+1, k+\frac{1}{2}}+w_{i, j+1, k-\frac{1}{2}}-w_{i, j, k+\frac{1}{2}}-w_{i, j, k+\frac{1}{2}}-w_{i, j, k-\frac{1}{2}}}{\delta y}\right)\right] .
\end{aligned}
$$

As equações correspondentes aos demais casos neste tipo de superfícies são construídas de forma análoga.

Para a aplicação das condições de contorno no contorno rígido, considera-se, sem perda de generalidade, o caso particular ilustrado na Figura 4.6 (os outros casos são feitos de maneira análoga). Nessa figura, as velocidades tangenciais correspondem a $u$ e $w$, e a variável $\phi$ representa uma das variáveis turbulentas $\kappa, \varepsilon$ ou $\nu_{t}$. Neste trabalho, diferentemente de como é feito na literatura, as condições de contorno no caso de escoamentos turbulentos são aplicadas de uma maneira particular, isto é, nas células adjacentes ao contorno rígido aplica-se normalmente as equações médias de Reynolds (2.64)-(2.66) para a determinação das velocidades, e para a obtenção das variáveis turbulentas aplicam-se as leis de parede discutidas na subseção 2.5.4.

Para o cálculo das variáveis turbulentas $\kappa$ e $\varepsilon$ adjacentes ao contorno rígido, primeiramente faz-se necessário conhecer o valor da velocidade de atrito $u^{*}$. Assim, dependendo do valor do número de Reynolds local $y^{+}$dado por (2.83), a velocidade de atrito $u^{*}$ é obtida a partir das relações $(2.86)$ ou (2.87). Admitindo-se que $y^{+}$está na camada viscosa, os cálculos são inicializados determinando-se o número Reynolds crítico $y_{c}^{+}$. Se $y^{+}$for menor que $y_{c}^{+}$, emprega-se (2.86); caso contrário, utiliza-se a relação (2.87). No caso em que $u^{*}$ é obtida por (2.87), o método de Newton-Raphson é aplicado, na qual impõe-se $u^{*}=11.60$ como aproximação inicial e $1.0 \times 10^{-14}$ como critério de parada.

Uma vez calculada a velocidade de atrito $u^{*}$, as variáveis turbulentas $\kappa$ e $\varepsilon$ na subcamada inercial (turbulenta), aplicadas no ponto $(i, j, k)$, são calculadas por

$$
\kappa_{i, j, k}=\operatorname{Re} \frac{\tau_{\omega}}{\sqrt{C_{\mu}}} \quad \text { e } \quad \varepsilon_{i, j, k}=R e \frac{\tau_{\omega} u^{*}}{K y} .
$$




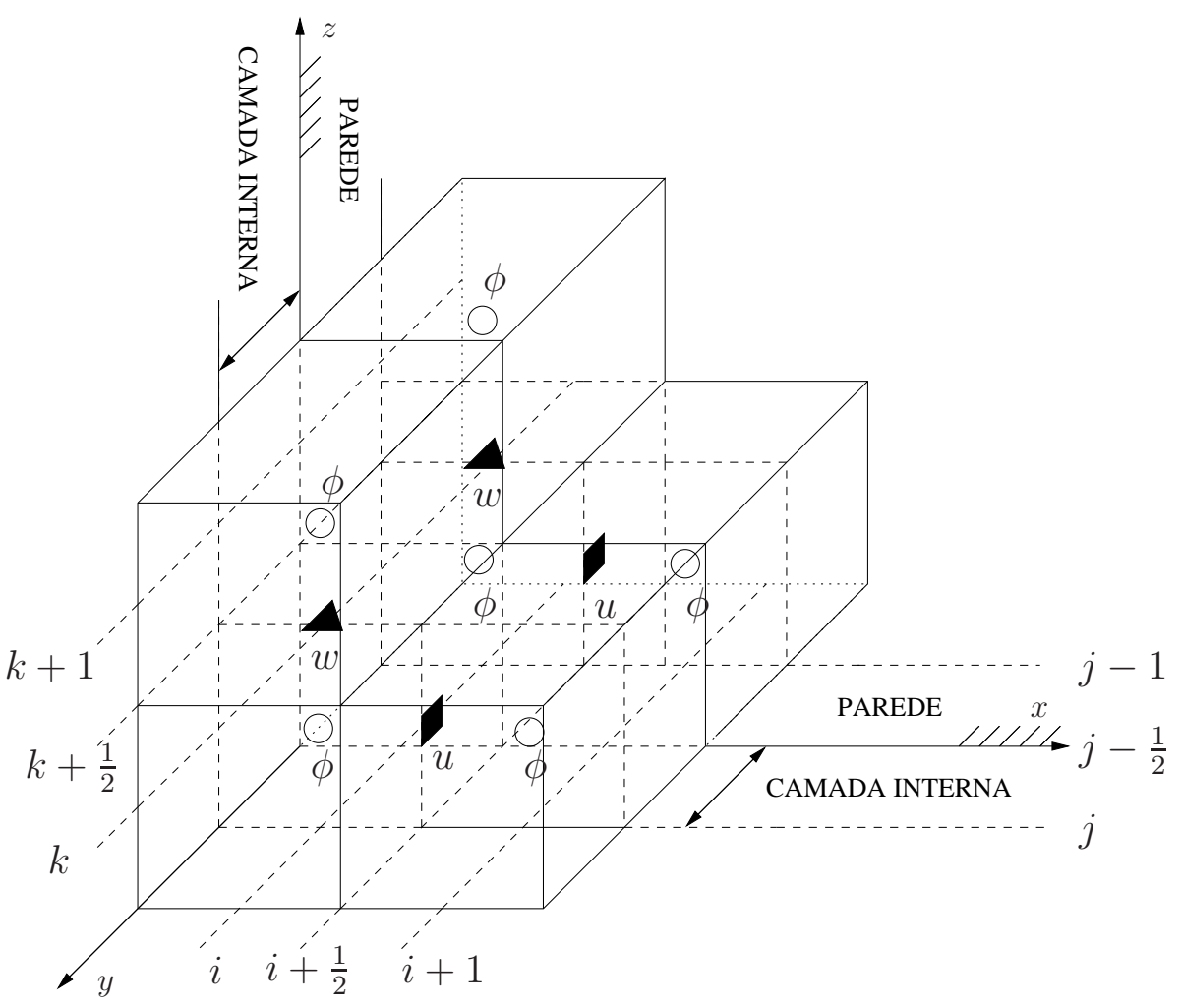

Figura 4.6: Representação esquemática de células adjacentes ao contorno rígido, mostrando a camada interna adjacente às paredes e as células fictícias.

e, na subcamada viscosa, pelas expressões

$$
\kappa_{i, j, k}=R e \frac{\tau_{\omega}}{\sqrt{C_{\mu}}}\left(\frac{y^{+}}{y_{c}^{+}}\right)^{2} \quad \text { e } \quad \varepsilon_{i, j, k}=\sqrt{\frac{1}{R e}} \frac{\left(\kappa_{i, j, k}\right)^{\frac{3}{2}}}{l^{*}},
$$

em que $l^{*}$ é a escala de comprimento dado por (2.89).

A viscosidade turbulenta, $\nu_{t}$, avaliada no ponto $(i, j, k)$, é calculada por

$$
\nu_{t i, j, k}=C_{\mu} \kappa_{i, j, k} T_{i, j, k}=C_{\mu} \frac{\kappa_{i, j, k}^{2}}{\varepsilon_{i, j, k}} .
$$

As velocidades que estão fora do domínio computacional, $u_{i+\frac{1}{2}, j-1, k}$ e $w_{i, j-1, k+\frac{1}{2}}$, são calculadas aproximando-se (2.92) por diferenças centrais nos pontos $\left(i+\frac{1}{2}, j-\frac{1}{2}, k\right)$ e $\left(i, j-\frac{1}{2}, k+\frac{1}{2}\right)$, respectivamente. A velocidade tangencial $u$ avaliada no ponto $\left(i+\frac{1}{2}, j-1, k\right)$ é dada por

$$
u_{i+\frac{1}{2}, j-1, k}=u_{i+\frac{1}{2}, j, k}-\operatorname{sinal}\left(u_{i+\frac{1}{2}, j, k}\right) \frac{\operatorname{Re}_{\omega} \delta y}{\left(1+\nu_{t i+\frac{1}{2}, j-\frac{1}{2}, k}\right)},
$$


em que $\operatorname{sinal}\left(u_{i+\frac{1}{2}, j, k}\right)$ é o sinal da velocidade tangencial $u_{i+\frac{1}{2}, j, k} \mathrm{e}$

$$
\nu_{t i+\frac{1}{2}, j-\frac{1}{2}, k}=0.25\left(\nu_{t i, j-1, k}+\nu_{t i, j, k}+\nu_{t i+1, j-1, k}+\nu_{t i+1, j, k}\right) .
$$

A outra velocidade tangencial, $w$, avaliada no ponto $\left(i, j-\frac{1}{2}, k+\frac{1}{2}\right)$ é dada por

$$
w_{i, j-1, k+\frac{1}{2}}=w_{i, j, k+\frac{1}{2}}-\operatorname{sinal}\left(w_{i, j, k+\frac{1}{2}}\right) \frac{\operatorname{Re} \tau_{\omega} \delta y}{\left(1+\nu_{t i, j-\frac{1}{2}, k+\frac{1}{2}}\right)}
$$

em que $\operatorname{sinal}\left(w_{i, j, k+\frac{1}{2}}\right)$ é o sinal da velocidade tangencial $w_{i, j, k+\frac{1}{2}} \mathrm{e}$

$$
\nu_{t i, j-\frac{1}{2}, k+\frac{1}{2}, k}=0.25\left(\nu_{t i, j-1, k}+\nu_{t i, j, k}+\nu_{t i, j-1, k+1}+\nu_{t i, j, k+1}\right) .
$$




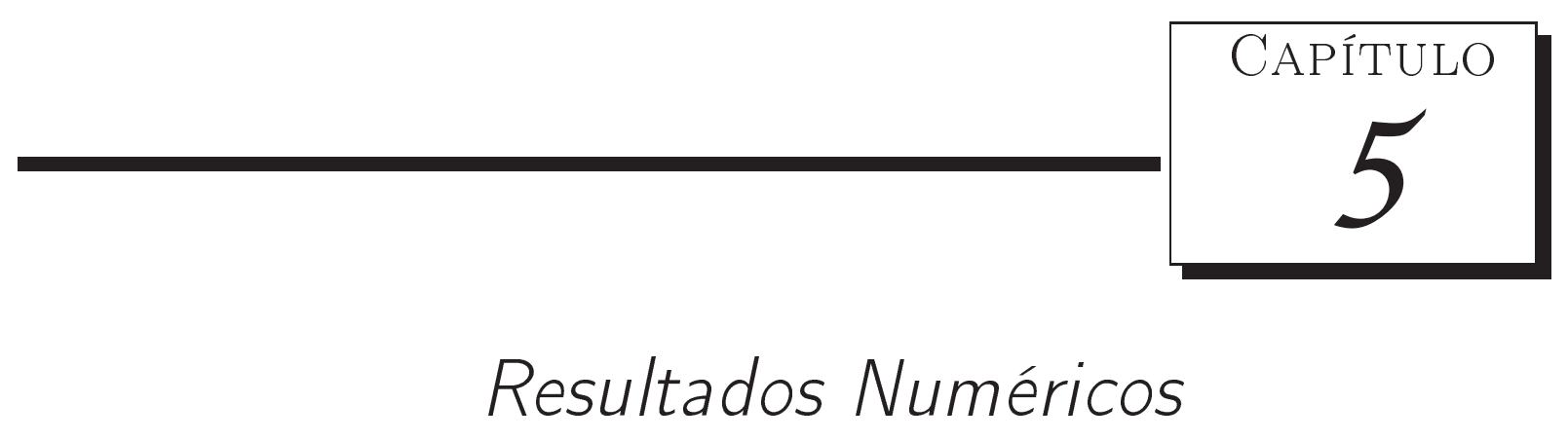

Neste capítulo são discutidos os resultados numéricos para a solução de três tipos de EDPs complexas: (i) leis de conservação 1D da dinâmica dos gases; (ii) equações de Navier-Stokes 2D e 3D para a simulação de escoamentos incompressíveis laminares com superfícies livres; e (iii) equações médias de Navier-Stokes $2 \mathrm{D}$ e $3 \mathrm{D}$, mais modelagem $\kappa-\varepsilon$, para a simulação de escoamentos incompressívies turbulentos com superfícies livres.

\subsection{Simulação de leis de conservação 1D}

Nesta seção são apresentados resultados de simulações numéricas das leis de conservação 1D. Objetiva-se mostrar que o novo esquema ADBQUICKEST, descrito na seção 3.5, é efetivo em várias situações de EDPs lineares e não lineares. Para isso, são considerados os seguintes problemas transientes: advecção de escalares, problemas de Riemann para as equações de Burgers, de Euler e águas rasas. Esses problemas são considerados na literatura importantes testes para avaliar o desempenho, a robustez e a precisão de esquemas numéricos.

\subsubsection{Equação linear de advecção}

Aqui, são apresentados os resultados da solução numérica da equação (2.1) com $a=1$ e condições iniciais distintas.

Problema teste 1: Nesse primeiro teste o esquema ADBQUICKEST é aplicado para resolver um problema com gradientes elevados. O problema, também simulado por Harten 
[55], consiste em resolver a equação (2.1) suplementada com a condição inicial

$$
u_{0}(x)= \begin{cases}-x \sin \left(\frac{3 \pi x^{2}}{2}\right), & -1 \leq x \leq-\frac{1}{3}, \\ |\sin (2 \pi x)|, & |x|<\frac{1}{3} \\ 2 x-1-\frac{1}{6} \sin (3 \pi x), & \frac{1}{3} \leq x \leq 1\end{cases}
$$

Este problema tem sido um bom teste combinando descontinuidades e regiões suave. Para a simulação deste problema foram utilizados os seguintes dados: malha de 200 células computacionais, dois números de Courant $(\theta=0.3$ e $\theta=0.8)$ e tempo final $t=1.0$.

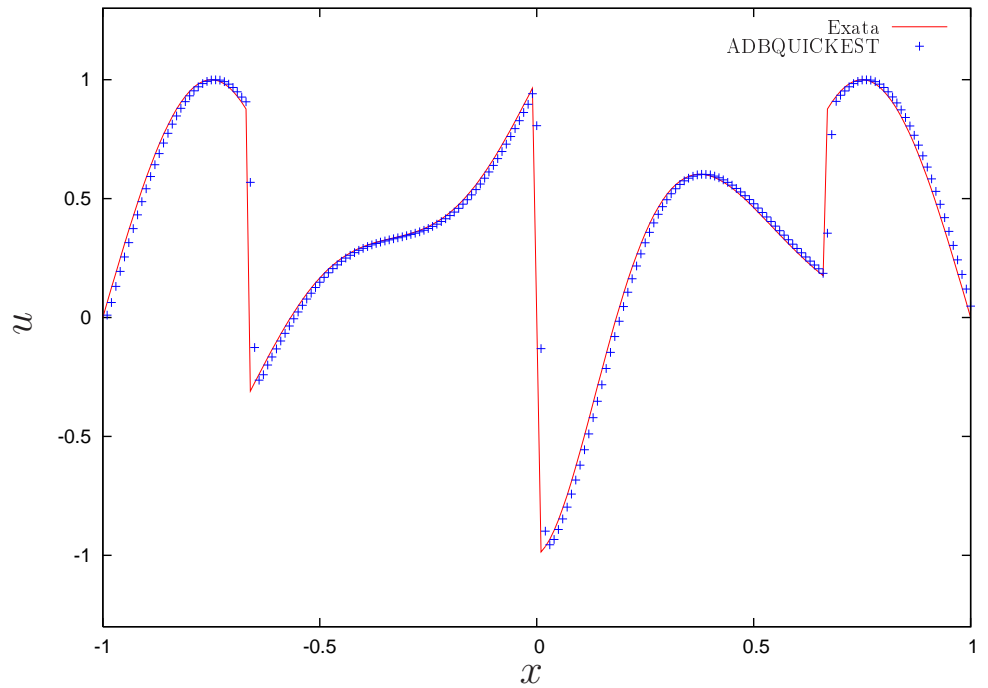

Figura 5.1: Soluções exata e numérica da equação linear de advecção usando as condições iniciais (5.1) $\operatorname{com} \theta=0.3$.

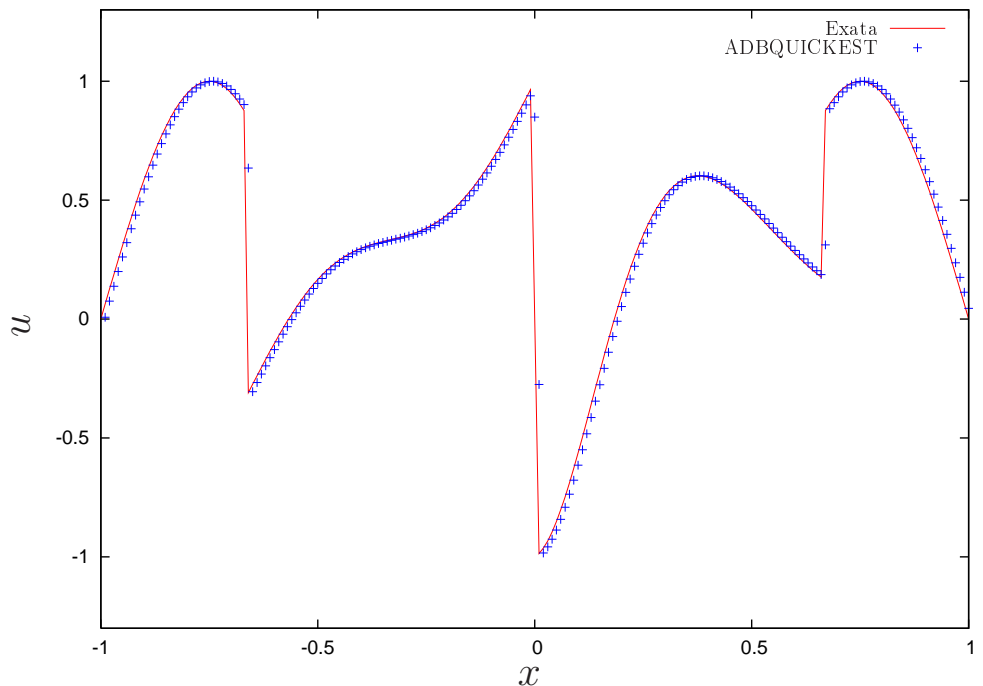

Figura 5.2: Soluções exata e numérica da equação linear de advecção usando as condições iniciais (5.1) $\operatorname{com} \theta=0.8$. 
As Figuras 5.1 e 5.2 mostram a comparação entre a solução exata e os resultados númericos obtidos com o esquema ADBQUICKEST. Observa-se claramente a partir dessas figuras, que o esquema ADBQUICKEST reproduziu resultados satisfatórios sobre todo o domínio, capturando a região de descontinuidade.

A Figura 5.3 representa o erro medido nas normas $L_{1}, L_{2}$ e $L_{\infty}$ e em três malhas entre a solução exata e os dados numéricos utilizando $\theta=0.8$ e tempo final $t=1.0$. Pode-se observar que, para este problema envolvendo gradientes elevados, a ordem de precisão do esquema ADBQUICKEST é, como era de se esperar, de primeira ordem. E esta observação está em concordância com os dados por Titarev e Toro [139].

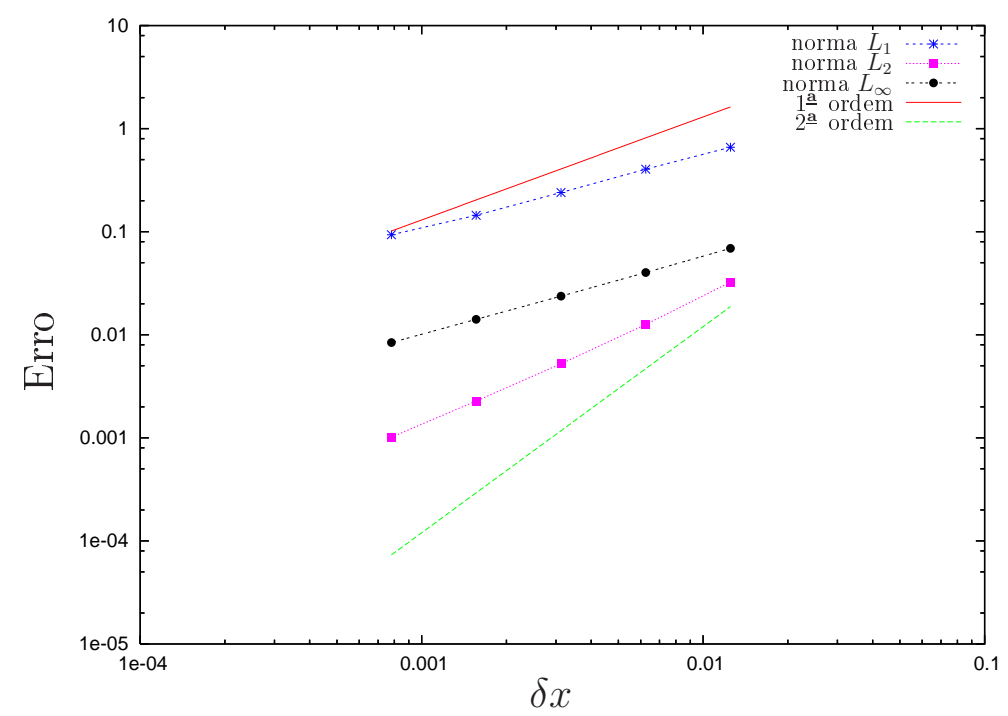

Figura 5.3: Erro na solução numérica da equação linear de advecção (2.1) com a condição inicial $(5.1)$.

Problema teste 2: O segundo teste considerado para examinar o desempenho do esquema ADBQUICKEST é a equação (2.1) com $0 \leq x \leq 1$ e a condição inicial

$$
u_{0}(x)= \begin{cases}\exp \left[\left(\frac{-\log 2}{9}\right)(200(x-0.1))^{2}\right], & 0.05 \leq x<0.15, \\ \sin \left(\pi\left(\frac{x-0.25}{0.1}\right)\right)^{4}, & 0.25<x<0.35, \\ 1, & 0.45<x<0.55, \\ 1-\frac{|x-0.07|}{0.05}, & 0.65 \leq x \leq 0.75, \\ \sqrt{1-\left(\frac{x-0.9}{0.05}\right)^{2}}, & 0.85<x<0.95,\end{cases}
$$

que compreende a perfis interessantes para investigar características diferentes do esquema, a saber: (i) um seno e uma exponencial, para acessar a precisão do esquema em regiões suaves; (ii) um retângulo, para testar a resolução nas proximidades de descontinuidades; (iii) um triângulo, 
para acessar o aparamento de picos ou vales; e (iv) uma semi-elipse, para avaliar o esquema em regiões de variações súbitas nos gradientes.

Os seguintes dados para a simulação deste problema foram considerados: malha uniforme de 200 células computacionais, dois números de Courant $(\theta=0.3$ e $\theta=0.8)$ e tempo final $t=1.0$. As Figuras 5.4 e 5.5 mostram comparação entre a solução exata e os resultados númericos com o ADBQUICKEST. Neste teste, como pode-se observar a partir dessas figuras, o esquema forneceu resultados satisfatórios.

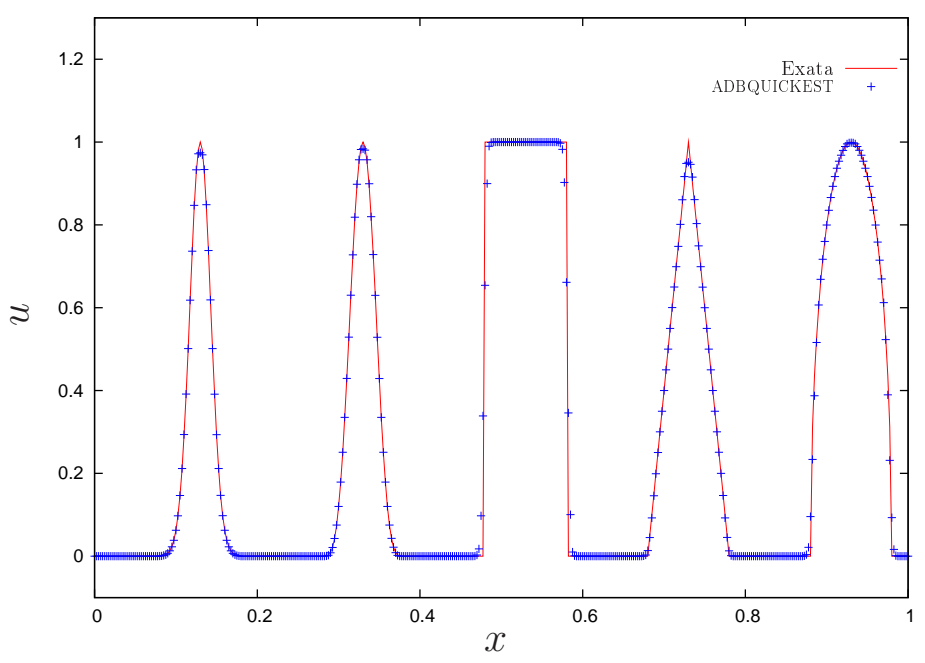

Figura 5.4: Soluções exata e numérica da equação linear de advecção usando as condições iniciais (5.2) $\operatorname{com} \theta=0.3$.

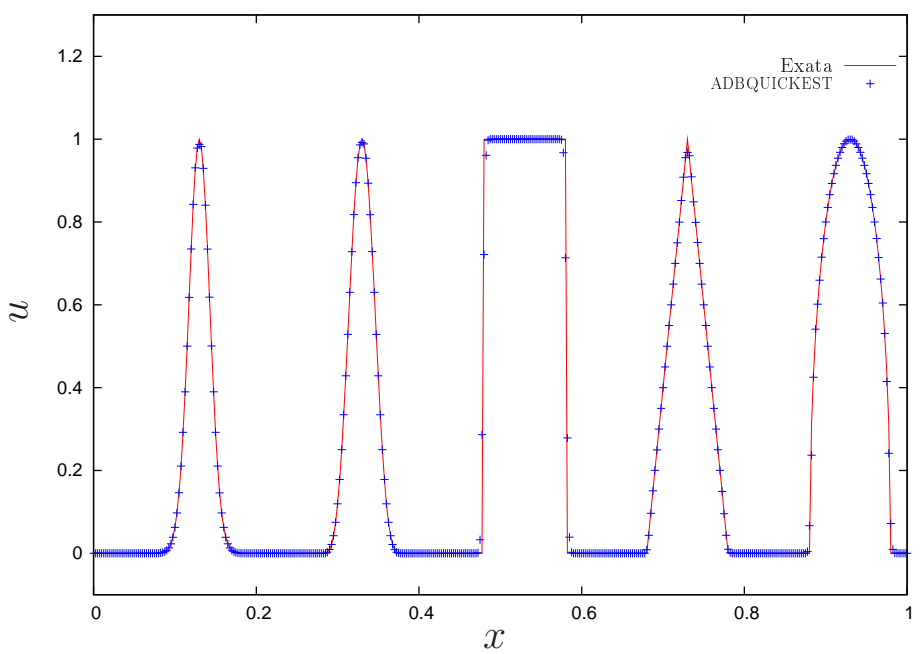

Figura 5.5: Soluções exata e numérica da equação linear de advecção usando as condições iniciais (5.2) $\operatorname{com} \theta=0.8$.

Problema teste 3: A fim de testar o comportamento do esquema em problemas mais complexos, é apresentado neste teste a solução numérica para o problema proposto por Wei e 
Gu [156] (a famosa forma "W-shape"), que corresponde resolver a equação (2.1) com a condição inicial

$$
u_{0}(x)= \begin{cases}1, & 0 \leq x \leq 0.2 \\ 4 x-\frac{3}{5}, & 0.2<x \leq 0.4 \\ -4 x+\frac{13}{5}, & 0.4<x \leq 0.6 \\ 1, & 0.6<x \leq 0.8 \\ 0, & \text { caso contrário. }\end{cases}
$$

A solução desse problema apresenta descontinuidades severas difíceis de se resolver (ver Toro [145], Wei e $\mathrm{Gu}[156])$.

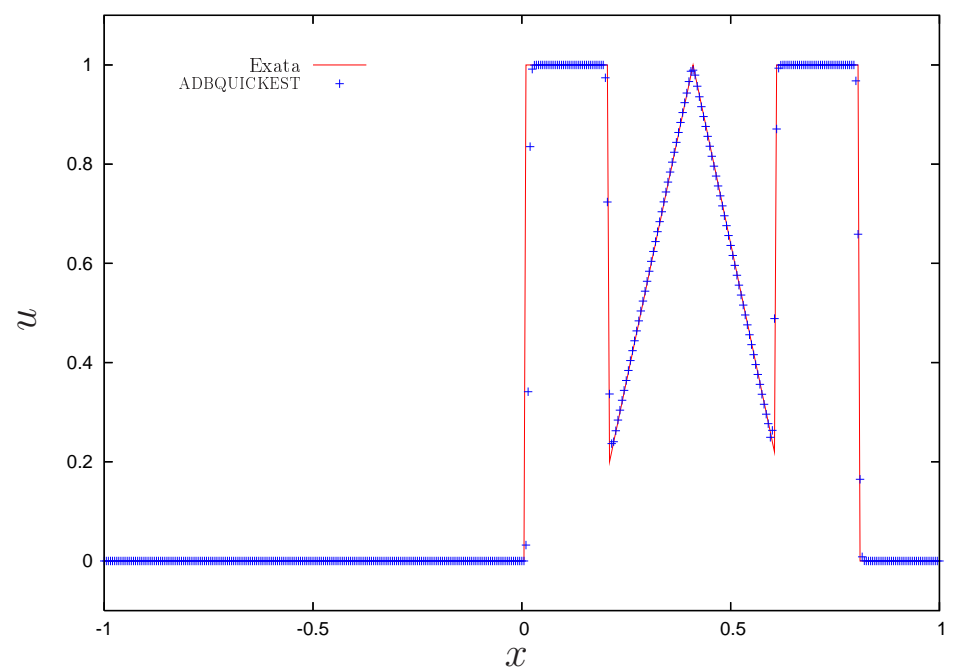

Figura 5.6: Soluções exata e numérica da equação linear de advecção usando as condições iniciais (5.3) $\operatorname{com} \theta=0.3$.

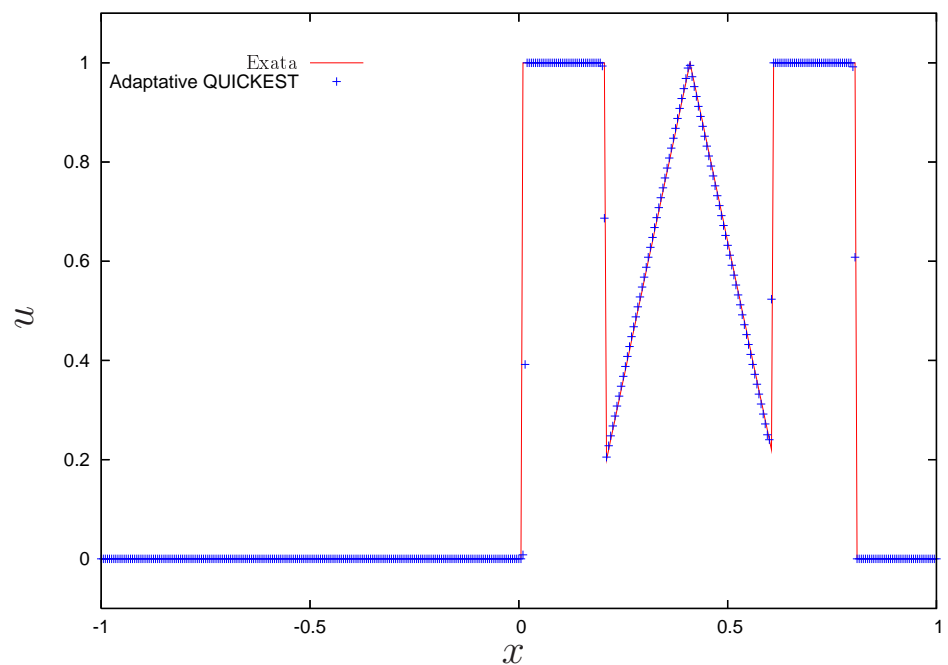

Figura 5.7: Soluções exata e numérica da equação linear de advecção usando as condições iniciais (5.3) $\operatorname{com} \theta=0.8$. 
Para a simulação deste problema foram utilizadas: malha uniforme de 200 células computacionais, dois números de Courant $(\theta=0.3$ e $\theta=0.8)$ e tempo final $t=1.0$. A solução exata e os resultados numéricos obtidos com o esquema ADBQUICKEST são apresentados nas Figuras 5.6 e 5.7. A partir dessas figuras, vê-se claramente que os picos e os vales são capturados satisfatoriamente pelo método numérico usando o ADBQUICKEST, sem apresentar qualquer vestígios de oscilação.

Problema teste 4: Este teste foi proposto com o objetivo de se comparar o esquema ADBQUICKEST com os esquema bem conhecidos na literatura CUBISTA (Alves et al. [2]), WACEB (Song et al. [126]) e SMART (Gaskell e Lau [47]), todos aplicados a equação (2.1), sobre o domínio $[-\pi, \pi]$, com a condição inicial suave $u_{0}(x)=\sin (x)$ (Herrmann et al. [58]).

Tabela 5.1: Teste de convergência: erros nas normas $L_{1}, L_{2}$ e $L_{\infty}$ e estimativa da ordem de convergência para os quatros esquemas com $u_{0}=\sin (x),-\pi \leq x \leq \pi$.

\begin{tabular}{|l|r|c|c|c|c|cc|}
\hline Esquema & $N$ & \multicolumn{2}{|c|}{$L_{1}$} & \multicolumn{2}{c|}{$L_{2}$} & \multicolumn{2}{c|}{$L_{\infty}$} \\
\cline { 2 - 8 } & & Erro & Ordem & Erro & Ordem & Erro & Ordem \\
\hline ADBQUICKEST & 20 & $0.2736 \mathrm{e}-1$ & - & $0.3182 \mathrm{e}-1$ & - & $0.5287 \mathrm{e}-1$ & - \\
& 40 & $0.7997 \mathrm{e}-2$ & 1.775 & $0.1121 \mathrm{e}-1$ & 1.505 & $0.2580 \mathrm{e}-1$ & 1.092 \\
& 80 & $0.2521 \mathrm{e}-2$ & 1.665 & $0.4532 \mathrm{e}-2$ & 1.306 & $0.1200 \mathrm{e}-1$ & 1.047 \\
& 160 & $0.7952 \mathrm{e}-3$ & 1.664 & $0.1821 \mathrm{e}-2$ & 1.315 & $0.6231 \mathrm{e}-2$ & 0.945 \\
& 320 & $0.2467 \mathrm{e}-3$ & 1.688 & $0.7394 \mathrm{e}-3$ & 1.300 & $0.2920 \mathrm{e}-2$ & 1.093 \\
\hline CUBISTA & 20 & $0.3728 \mathrm{e}-1$ & - & $0.4109 \mathrm{e}-1$ & - & $0.6456 \mathrm{e}-1$ & - \\
& 40 & $0.1151 \mathrm{e}-1$ & 1.695 & $0.1539 \mathrm{e}-1$ & 1.417 & $0.3321 \mathrm{e}-1$ & 0.937 \\
& 80 & $0.3877 \mathrm{e}-2$ & 1.582 & $0.6464 \mathrm{e}-2$ & 1.250 & $0.1804 \mathrm{e}-1$ & 0.880 \\
& 160 & $0.1233 \mathrm{e}-2$ & 1.652 & $0.2599 \mathrm{e}-2$ & 1.314 & $0.9092 \mathrm{e}-2$ & 0.988 \\
& 320 & $0.4903 \mathrm{e}-3$ & 1.688 & $0.1999 \mathrm{e}-2$ & 1.241 & $0.4460 \mathrm{e}-2$ & 1.027 \\
\hline WACEB & 20 & $0.2855 \mathrm{e}-1$ & - & $0.3198 \mathrm{e}-1$ & - & $0.5016 \mathrm{e}-1$ & - \\
& 40 & $0.8756 \mathrm{e}-2$ & 1.705 & $0.1139 \mathrm{e}-1$ & 1.489 & $0.2287 \mathrm{e}-1$ & 1.133 \\
& 80 & $0.2762 \mathrm{e}-2$ & 1.665 & $0.4318 \mathrm{e}-2$ & 1.402 & $0.1250 \mathrm{e}-1$ & 0.871 \\
& 160 & $0.8811 \mathrm{e}-3$ & 1.648 & $0.1663 \mathrm{e}-2$ & 1.377 & $0.7031 \mathrm{e}-2$ & 0.830 \\
& 320 & $0.2852 \mathrm{e}-3$ & 1.627 & $0.7534 \mathrm{e}-3$ & 1.142 & $0.3650 \mathrm{e}-2$ & 0.946 \\
\hline SMART & 20 & $0.1678 \mathrm{e}-1$ & - & $0.2070 \mathrm{e}-1$ & - & $0.6662 \mathrm{e}-1$ & - \\
& 40 & $0.6516 \mathrm{e}-2$ & 1.365 & $0.1027 \mathrm{e}-1$ & 1.021 & $0.3493 \mathrm{e}-1$ & 0.931 \\
& 80 & $0.2368 \mathrm{e}-2$ & 1.460 & $0.4544 \mathrm{e}-2$ & 1.176 & $0.1655 \mathrm{e}-1$ & 1.078 \\
& 160 & $0.8069 \mathrm{e}-3$ & 1.553 & $0.1758 \mathrm{e}-2$ & 1.370 & $0.9482 \mathrm{e}-2$ & 0.804 \\
& 320 & $0.3050 \mathrm{e}-3$ & 1.403 & $0.7621 \mathrm{e}-3$ & 1.205 & $0.4990 \mathrm{e}-2$ & 0.926 \\
\hline
\end{tabular}

Cincos malhas ( $N=20,40,80,160$ e 320 células computacionais) e número de Courant $\theta=0.5$ foram utilizados nesta comparação, e os erros nas normas $L_{1}, L_{2}$ e $L_{\infty}$, bem como estimativas para a ordem de convergência, estão apresentados na Tabela 5.1. A partir desta tabela, os dados numéricos indicam que, em tais normas, o esquema ADBQUICKEST apresenta 
ordem de convergência superior a dos esquemas CUBISTA, WACEB e SMART.

Problema teste 5: Neste teste é apresentado um estudo de convergência semelhante ao problema teste 4 , com a condição inicial $u_{0}(x)=\sin ^{4}(\pi x)$ para $x \in[-2,2]$ (ver detalhes em Balsara e Shu [10]). Conforme comentado por Balsara e Shu, essa condição inicial é mais acentuada que aquela apresentada no teste 4. Por esta razão, Rogerson e Meiburg [115] mostraram que os esquemas tipo ENO sofrem redução na precisão para este tipo de problema suave. Ao contrário, as estimativas para a ordem de convergência obtidas com o esquema ADBQUICKEST sofreu um acrescémo, como pode ser visto na Tabela 5.2 onde os erros nas normas $L_{1}, L_{2}$ e $L_{\infty}$ são apresentados. Além disso, comparados com os resultados dos esquemas CUBISTA, WACEB e SMART, pode ser visto por essa mesma tabela que, na norma $L_{1}$, o esquema ADBQUICKEST manteve segunda ordem, ao passo que os outros esquemas forneceram estimativas inferior a 2. E nas outras normas, os quatros esquemas forneceram resultados semelhantes. Nota-se também, por esta mesma tabela, que houve melhoria na ordem de precisão do ADBQUICKEST quando comparado a ordem atingida no problema teste 4 .

Tabela 5.2: Teste de convergência: erros nas normas $L_{1}, L_{2}$ e $L_{\infty}$ e estimativa da ordem de convergência para os quatros esquemas com $u_{0}(x)=\sin ^{4}(\pi x),-2 \leq x \leq 2$.

\begin{tabular}{|l|r|c|c|c|c|cc|}
\hline Esquema & $N$ & \multicolumn{2}{|c|}{$L_{1}$} & \multicolumn{2}{c|}{$L_{2}$} & \multicolumn{2}{c|}{$L_{\infty}$} \\
\cline { 2 - 9 } & & Erro & Ordem & Erro & Ordem & Erro & Ordem \\
\hline ADBQUICKEST & 20 & $2.072 \mathrm{e}-1$ & - & $2.111 \mathrm{e}-1$ & - & 2.486 e-1 & - \\
& 40 & $4.722 \mathrm{e}-2$ & 2.133 & $5.433 \mathrm{e}-2$ & 1.958 & $8.409 \mathrm{e}-2$ & 1.564 \\
& 80 & $1.139 \mathrm{e}-2$ & 2.052 & $1.439 \mathrm{e}-2$ & 1.917 & $2.994 \mathrm{e}-2$ & 1.490 \\
& 160 & $2.368 \mathrm{e}-3$ & 2.266 & $3.998 \mathrm{e}-3$ & 1.848 & $1.057 \mathrm{e}-2$ & 1.502 \\
& 320 & $5.269 \mathrm{e}-4$ & 2.168 & $1.123 \mathrm{e}-3$ & 1.832 & $3.744 \mathrm{e}-3$ & 1.497 \\
\hline CUBISTA & 20 & $2.138 \mathrm{e}-1$ & - & $2.197 \mathrm{e}-1$ & - & $2.588 \mathrm{e}-1$ & - \\
& 40 & $5.665 \mathrm{e}-2$ & 1.916 & $6.148 \mathrm{e}-2$ & 1.837 & $9.408 \mathrm{e}-2$ & 1.450 \\
& 80 & $1.564 \mathrm{e}-2$ & 1.857 & $1.937 \mathrm{e}-2$ & 1.666 & $3.597 \mathrm{e}-2$ & 1.387 \\
& 160 & $4.404 \mathrm{e}-3$ & 1.828 & $6.213 \mathrm{e}-3$ & 1.640 & $1.384 \mathrm{e}-2$ & 1.378 \\
& 320 & $1.125 \mathrm{e}-3$ & 1.968 & $1.935 \mathrm{e}-3$ & 1.683 & $5.247 \mathrm{e}-3$ & 1.399 \\
\hline WACEB & 20 & $2.097 \mathrm{e}-1$ & - & $2.144 \mathrm{e}-1$ & - & $2.525 \mathrm{e}-1$ & - \\
& 40 & $4.539 \mathrm{e}-2$ & 2.208 & $5.373 \mathrm{e}-2$ & 1.996 & $8.423 \mathrm{e}-2$ & 1.584 \\
& 80 & $1.212 \mathrm{e}-2$ & 1.905 & $1.557 \mathrm{e}-2$ & 1.787 & $3.102 \mathrm{e}-2$ & 1.441 \\
& 160 & $3.257 \mathrm{e}-3$ & 1.896 & $4.712 \mathrm{e}-3$ & 1.724 & $1.120 \mathrm{e}-2$ & 1.469 \\
& 320 & $8.725 \mathrm{e}-4$ & 1.900 & $1.399 \mathrm{e}-3$ & 1.752 & $4.016 \mathrm{e}-3$ & 1.479 \\
\hline SMART & 20 & $2.036 \mathrm{e}-1$ & - & $2.105 \mathrm{e}-1$ & - & $2.412 \mathrm{e}-1$ & - \\
& 40 & $3.832 \mathrm{e}-2$ & 2.409 & $4.341 \mathrm{e}-2$ & 2.278 & $8.349 \mathrm{e}-2$ & 1.530 \\
& 80 & $8.293 \mathrm{e}-3$ & 2.208 & $9.949 \mathrm{e}-3$ & 2.125 & $2.977 \mathrm{e}-2$ & 1.488 \\
& 160 & $2.104 \mathrm{e}-3$ & 1.978 & $2.525 \mathrm{e}-3$ & 1.978 & $1.049 \mathrm{e}-2$ & 1.505 \\
& 320 & $5.859 \mathrm{e}-4$ & 1.844 & $7.736 \mathrm{e}-4$ & 1.706 & $3.646 \mathrm{e}-3$ & 1.525 \\
\hline
\end{tabular}




\subsubsection{Equação de Burgers}

Resolvida a equação linear de advecção usando diferentes condições iniciais, aqui são apresentados resultados numéricos para a equação não linear de Burgers 1D.

Problema teste 6: Neste teste, é considerada a equação (2.2) definida para $x \in[0,2 \pi]$ com a condição inicial dada por

$$
u_{0}(x)=1+\cos (x)
$$

e condições de contorno

$$
\left\{\begin{array}{l}
u(0, t)=1+\cos (t), \\
u(1, t)=\cos (t)+\cos (2 \pi) .
\end{array}\right.
$$

Os dados utilizados para a simulação deste teste foram: coeficiente de viscosidade $\nu=0.001$, 10000 células computacionais e número de Courant $\theta=0.5$. Os resultados numéricos foram computados utilizando-se o esquema ADBQUICKEST em intervalos de tempos de 0.25 até atingir o tempo final $t=3.25$, e estão mostrados na Figura 5.8. Pode-se observar a partir dessa figura, que os resultados obtidos estão em boa concordância com os resultados numéricos de Fatkullin e Hesthaven [37], ou seja, com o passar do tempo a solução numérica é propagada para direita com o decaimento da amplitude e formação de choque em regiões que apresentam gradientes elevados.

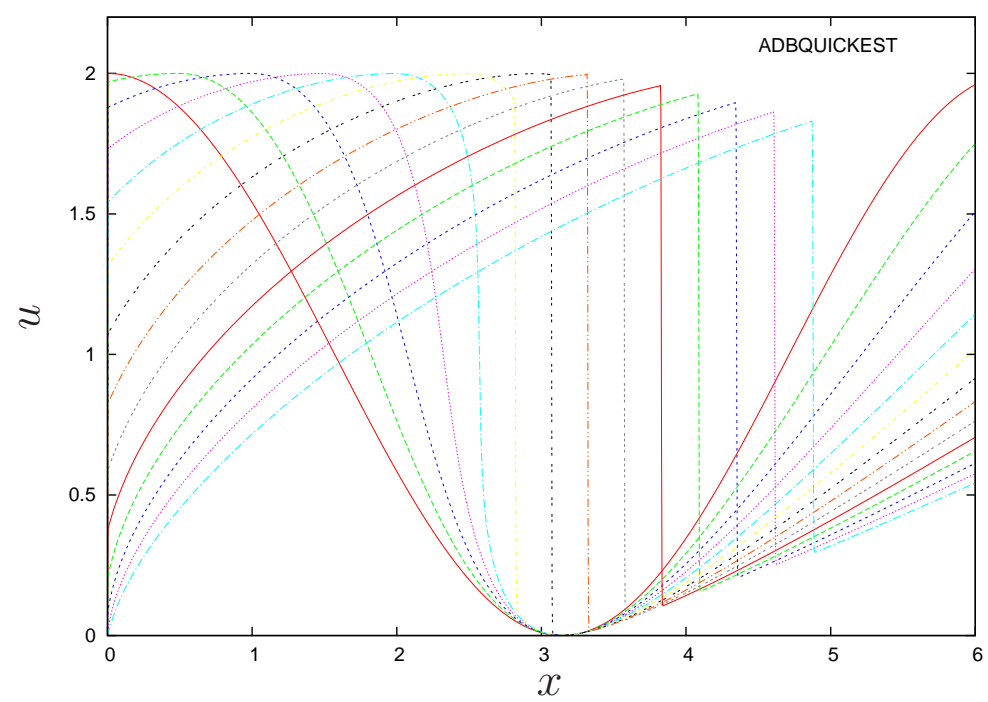

Figura 5.8: Resultados numéricos para a equação de Burgers com as condições (5.5) e (5.4) e $\nu=0.001$.

Problema teste 7: É considerado neste teste a equação $(2.2)$ definida para $x \in[0,1] \mathrm{e}$ suplementada com condição inicial

$$
u_{0}(x)=\sin (2 \pi x)
$$


e condições de contorno

$$
\left\{\begin{array}{l}
u(0, t)=0 \\
u(1, t)=0 .
\end{array}\right.
$$

Para resolver numericamente este problema, foram utilizados uma malha uniforme de 500 células computacionais e incremento temporal de 0.001. O esquema ADBQUICKEST foi aplicado usando-se o número de Courant $\theta=0.5$ e três valores de viscosidade: $\nu=0.01$, $\nu=0.001$ e $\nu=0.0001$. A Figura 5.9 mostra as soluções numéricas em seis tempos diferentes $(t=0.0,0.1,0.2,0.3,0.4,0.5)$. Em particular, pode-se observar nesta figura a formação do choque em $t=0.5$ e a capacidade do esquema ADBQUICKEST em reproduzir muito bem essa descontinuidade sem qualquer vestígios de oscilações espúrias. Nota-se, ainda, que os perfis transientes são totalmente simétricos em relação ao choque, que é uma característica desejável no procedimento numérico para este tipo de problema (ver Balsara e Shu [10]).

(a) $\nu=0.01$

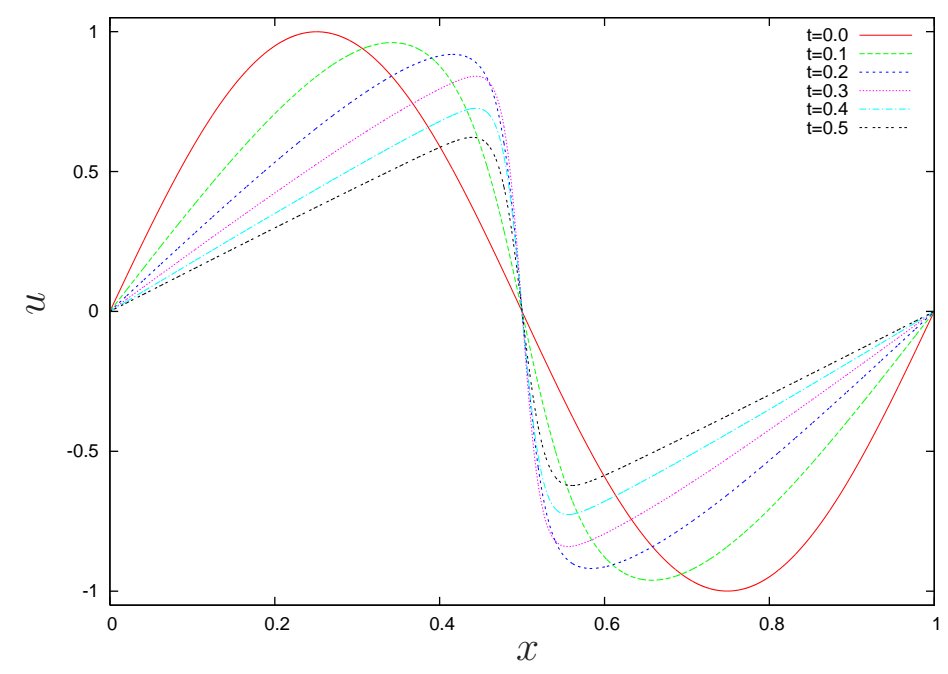

(b) $\nu=0.001$

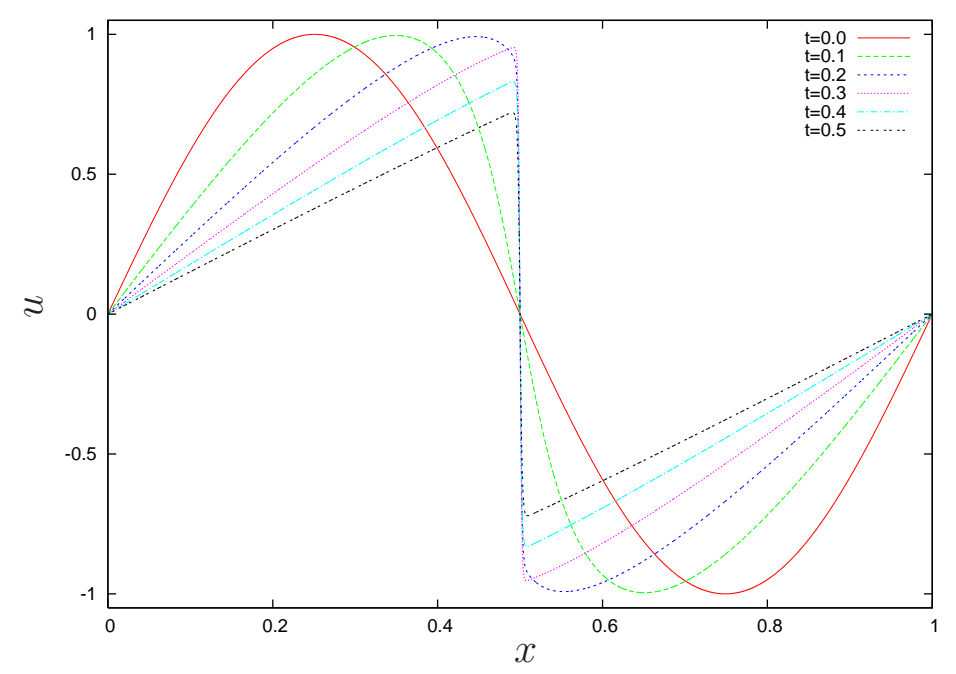


(c) $\nu=0.0001$

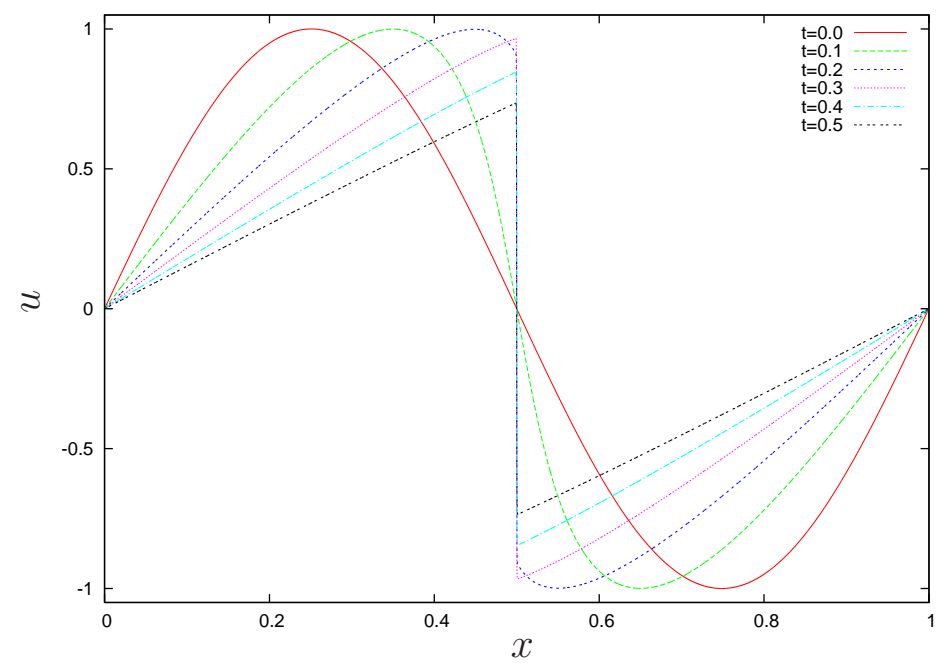

Figura 5.9: Resultados numéricos para a equação de Burgers com as condições (5.6)-(5.7).

\subsubsection{Sistemas de equações hiperbólicas}

Ao contrário dos testes anteriores, o esquema ADBQUICKEST é agora aplicado à sistemas de equações hiperbólicas, como as equações de Euler 1D, em particular, problemas do tubo de choque e as equações de águas rasas 1D, em particular, o problema "dam-break".

\subsubsection{Equações de águas rasas - problema "dam-break"}

Problema teste 8: Neste teste, o problema de Riemann para o sistema de equação (2.3) com $g=1$ é resolvido com condições iniciais

$$
u_{0}(x)=0
$$

para $u$ e para $h$,

$$
h_{0}(x)=\left\{\begin{array}{lll}
3, & \text { se } x \leq 0 \\
1, & \text { se } & x>0
\end{array}\right.
$$

e com condições homogêneas de Neumann no contorno (ver detalhes em LeVeque [82]).

Para a simulação do problema, foram escolhidos: o domínio $[-5,5]$, malha de 100 células computacionais, tempo final $t=2.0$ e número de Courant $\theta=0.8$. A solução de referência para este problema foi determinada pelo método de Godunov numa malha de 1000 células computacionais (ver LeVeque [82]). Os resultados dessa simulação, usando o ADBQUICKEST instalado no pacote CLAWPACK de LeVeque, e a solução de referência são apresentados na Figura 5.10 para a altura $h$ e na Figura 5.11 para a vazão $h u$. Pode-se observar nessas figuras que as soluções numéricas obtidas com o ADBQUICKEST estão próximas da solução de referência. As discrepâncias em algumas regiões são atribuídas à malha grossa empregada. 


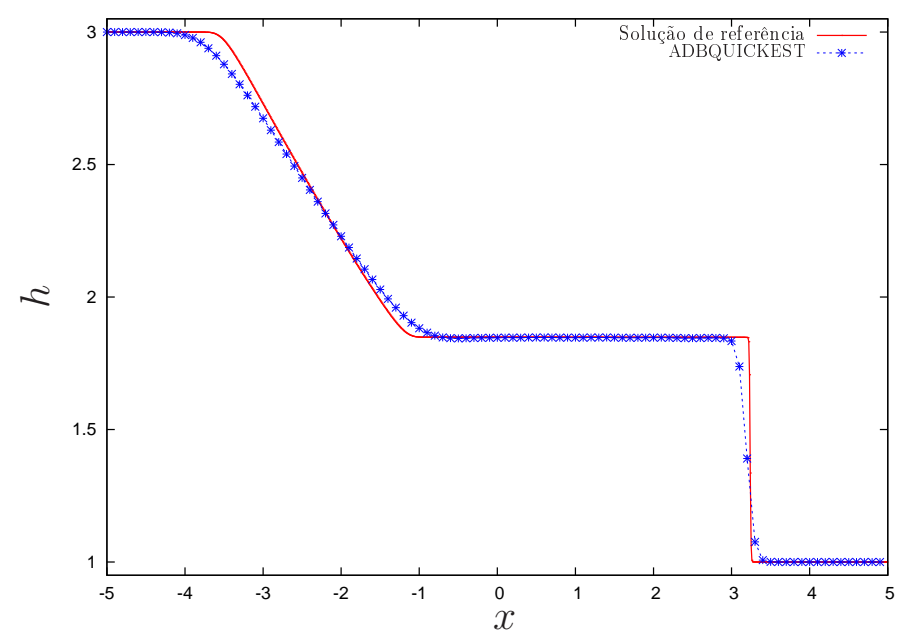

Figura 5.10: Soluções de referência e numérica para a profundidade $h$ no problema de águas rasas.

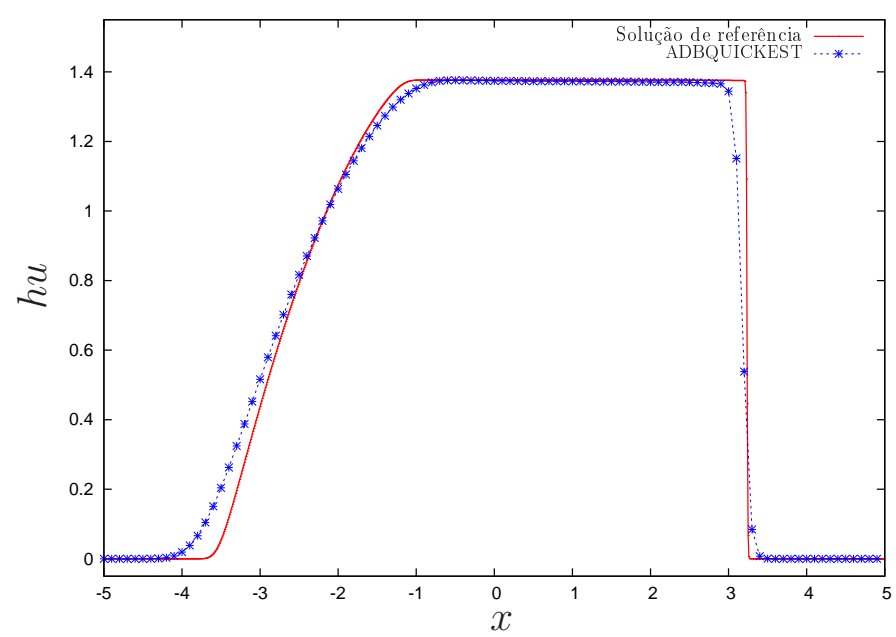

Figura 5.11: Soluções de referência e numérica da quantidade $h u$ para o problema de águas rasas.

\subsubsection{Equações de Euler - tubos de choque}

Tubos de choque vem sendo frequentemente utilizados na literatura para avaliar o desempenho, a robustez e a precisão de esquemas de alta resolução (ver, por exemplo, Titarev e Toro [139], Toro [145], Yang [162] entre outros).

Problema teste 9: Este problema de Riemann para as equações de Euler foi proposto por Sod [124], cuja solução consiste de uma rarefação a esquerda, um contato e um choque a direita. O problema é formulado pelo sistema de equações (2.4) sujeito à condição inicial

$$
\mathbf{u}_{0}(x)= \begin{cases}(1,0,1)^{T}, & x<0.5 \\ (0.125,0,0.1)^{T}, & x \geq 0.5\end{cases}
$$


Os seguintes dados para a simulação deste problema teste foram considerados: domínio $[0,1]$, malha uniforme composta de 400 células computacionais, dois números de Courant $(\theta=$ 0.2 e $\theta=0.6$ ) e tempo final $t=0.2$. Os resultados numéricos para a densidade $\rho$ obtidos com o ADBQUICKEST e a solução de referência (obtida aplicando-se o esquema "upwind" de $1^{\text {a }}$ ordem em uma malha de 1600 células) estão descritos nas Figuras 5.12 e 5.13.

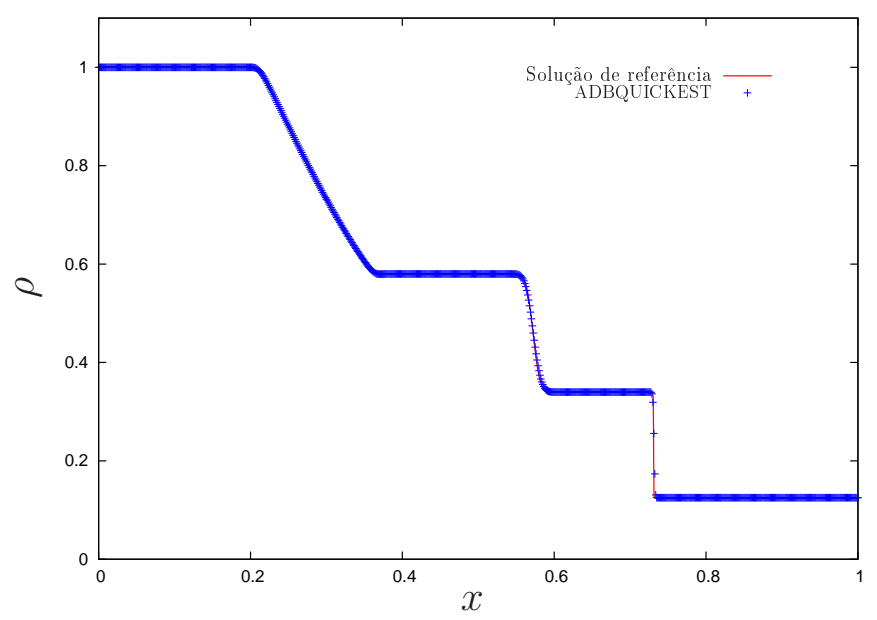

Figura 5.12: Perfil da densidade do problema teste $9 \operatorname{com} \theta=0.2$.

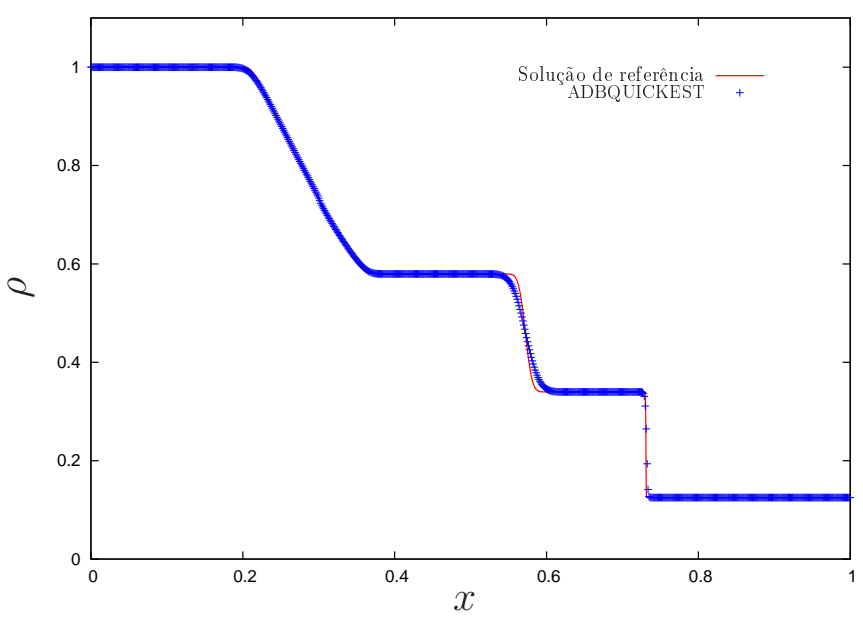

Figura 5.13: Perfil da densidade do problema teste $9 \operatorname{com} \theta=0.6$.

Nota-se por essas figuras que, de maneira geral, os resultados foram satisfatórios, sendo que o caso em que $\theta=0.2$ forneceu uma solução numérica bastante próxima da solução de referência.

Problema teste 10: Este problema teste, proposto por Lax [76] é formulado pelo sistema de equações (2.4) com $0 \leq x \leq 1$ e a condição inicial dada por

$$
\mathbf{u}_{0}(x)= \begin{cases}(0.445,0.3111,8.928)^{T}, & x<0.5 \\ (0.5,0,0.4275)^{T}, & x \geq 0.5\end{cases}
$$


A solução deste problema consiste de uma forte onda de choque, uma superfície de contato e uma onda de rarefação a esquerda. Os dados para a simulação deste problema de Riemann foram: malha uniforme de 400 células computacionais, dois números de Courant $(\theta=0.2$ e $\theta=0.6$ ) e tempo final $t=0.02$. Nas Figuras 5.14 e 5.15 estão apresentadas a solução de referência (computada de forma semelhante à do problema de Sod) e os resultados numéricos para a distribuição de densidade produzidos pelo esquema ADBQUICKEST. Vê-se claramente nessas figuras que, no geral, as soluções numéricas estão em boa concordância com a solução de referência, sendo que a solução numérica $\operatorname{com} \theta=0.2$ apresentou o melhor resultado.

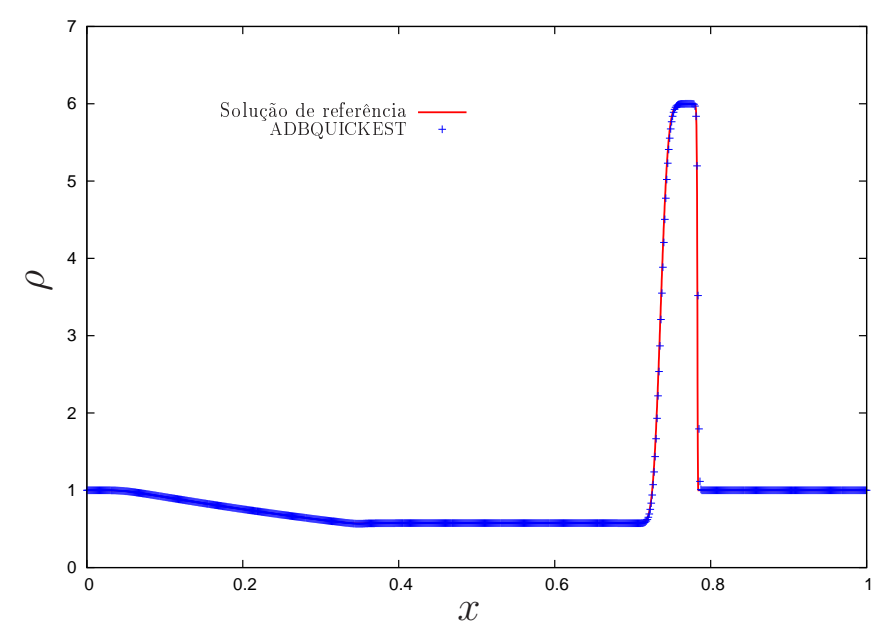

Figura 5.14: Perfil da densidade do problema teste $10 \operatorname{com} \theta=0.2$.

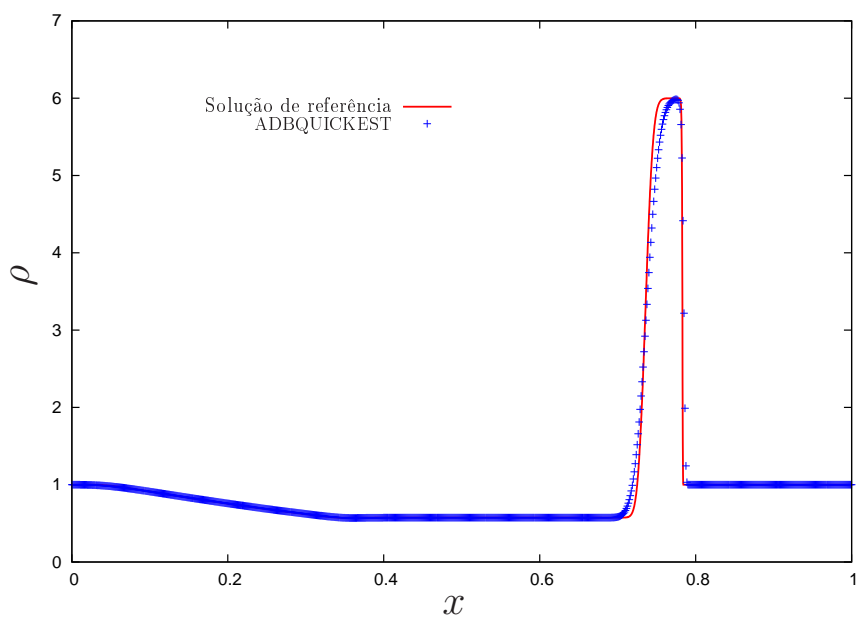

Figura 5.15: Perfil da densidade do problema teste $10 \operatorname{com} \theta=0.6$.

Problema teste 11: O próximo teste selecionado consiste em resolver o sistema (2.4) com $0 \leq x \leq 1$ e a seguinte condição inicial

$$
\mathbf{u}_{0}(x)= \begin{cases}(5.99924,195975,460.894)^{T}, & x<0.4 \\ (5.99242,-6.19633,46.0950)^{T}, & x \geq 0.4\end{cases}
$$


A solução deste teste consiste de três descontinuidades severas que se deslocam para à direita.

Para a simulação numérica deste teste com o esquema ADBQUICKEST, os seguintes dados foram empregados: malha uniforme de 400 células computacionais, dois números de Courant $(\theta=0.2$ e $\theta=0.6)$ e tempo final $t=0.035$. Os resultados numéricos para o perfil de densidade, $\rho$, e de referência (esquema "upwind" de $1^{\mathbf{a}}$ ordem com 4000 células) estão apresentados na Figura 5.16 para $\theta=0.2$ e na Figura 5.17 para $\theta=0.6$.

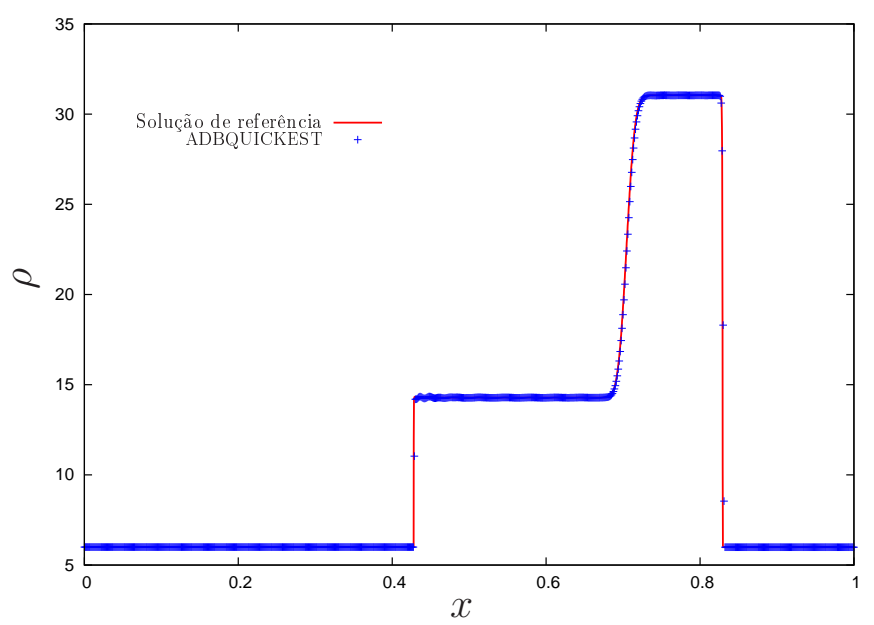

Figura 5.16: Perfil da densidade do problema teste $11 \operatorname{com} \theta=0.2$.

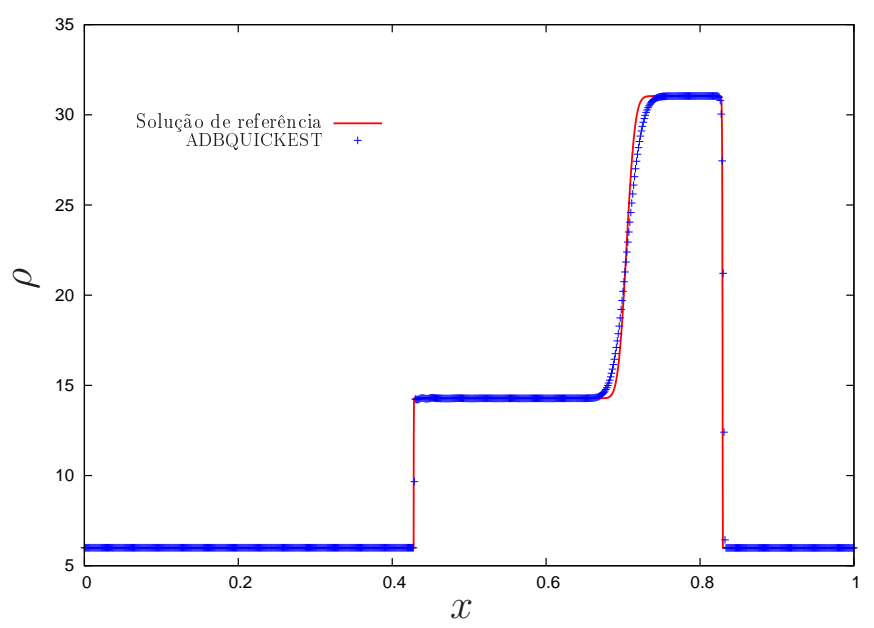

Figura 5.17: Perfil da densidade do problema teste $11 \operatorname{com} \theta=0.6$.

Novamente, pode-se ver a partir dessas figuras que os resultados numéricos foram bastante satisfatórios, sendo que o caso $\operatorname{com} \theta=0.2$ capturou melhor as descontinuidades.

Problema teste 12: Este teste é conhecido como tubo de choque de Shu-Osher [120], um modelo padrão de iteração choque/turbulência, que descreve uma onda acústica interagindo com uma pertubação na densidade tipo senoide (Balsara e Shu [10], Billet e Louedin [12], Levy 
et al. [84], Titarev e Toro [139]). Este problema é formulado pelo sistema de equações (2.4) sobre domínio $[-1,3]$ com a condição inicial dada por

$$
\mathbf{u}_{0}(x)= \begin{cases}(3.86,2.63,10.33)^{T}, & x<-0.8 \\ (1+0.2 \sin (5 x), 0,1)^{T}, & x \geq-0.8\end{cases}
$$

Para essa simulação desse tubo de choque, foram utilizados os seguintes dados: malha uniforme de 400 células computacionais, dois números de Courant $\theta=0.2$ e $\theta=0.6$ e tempo final $t=1.0$.

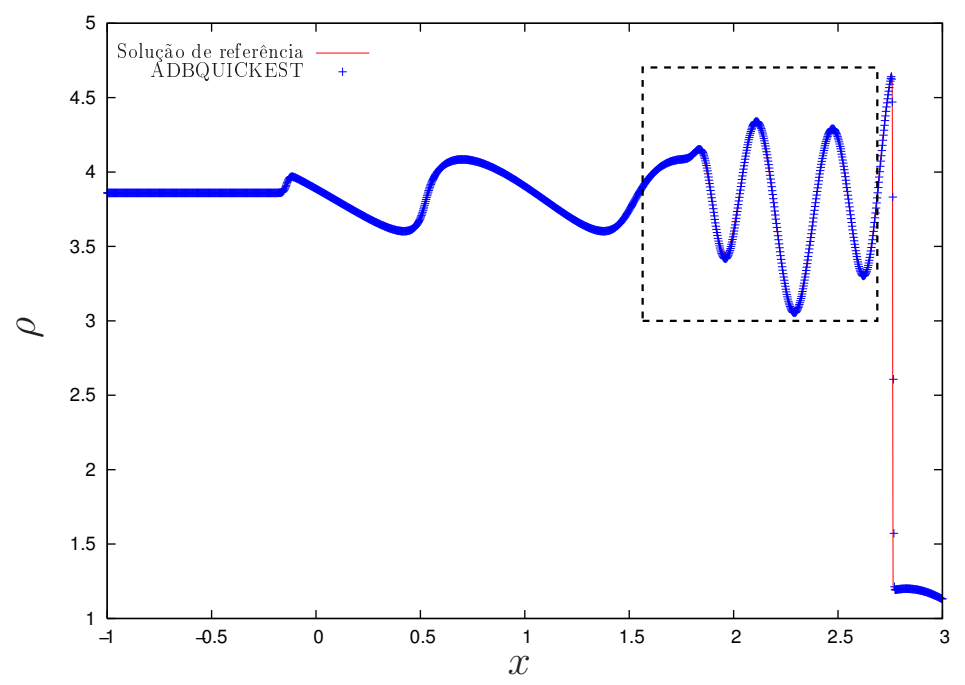

Figura 5.18: Perfil da densidade do problema teste $12 \operatorname{com} \theta=0.2$.

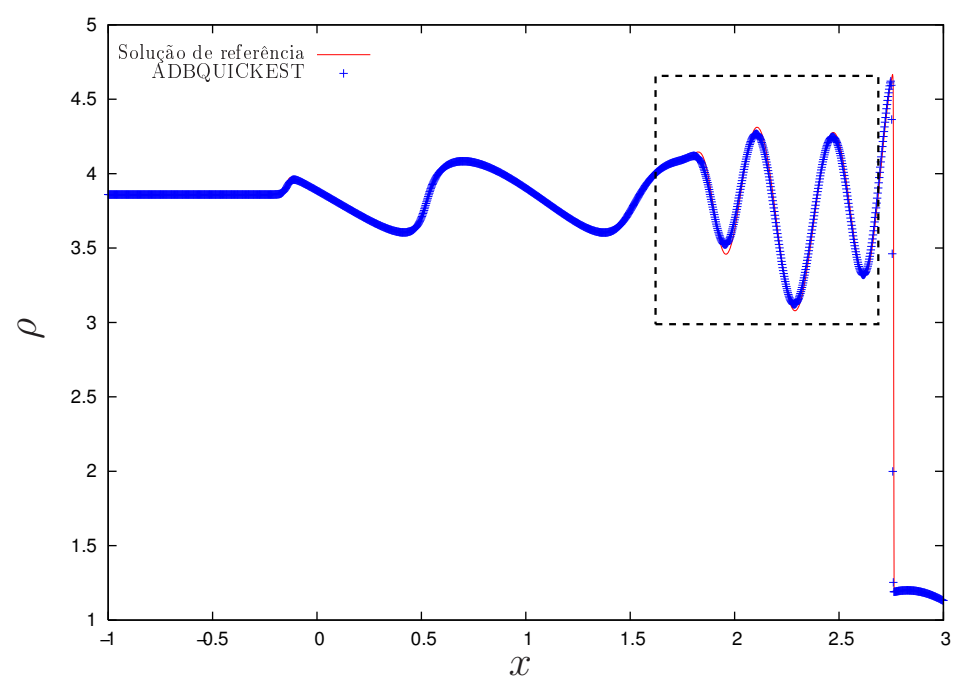

Figura 5.19: Perfil da densidade do problema teste $12 \operatorname{com} \theta=0.6$.

Apresentados nas Figuras 5.18 e 5.19 estão os resultados numéricos para a densidade obtidos com o esquema ADBQUICKEST e a solução de referência (esquema "upwind" de $1^{\mathrm{a}}$ ordem em 
uma malha de 1600 células). As Figuras 5.20 e 5.21 mostram a ampliação de ambas as soluções no caso $\theta=0.2$ e $\theta=0.6$, respectivamente. Pode ser visto nessas figuras que os resultados numéricos estão em harmonia com a solução de referência, especialmente próximo do ponto $x=2.0$, onde é a região mais crítica. Nota-se também, que os picos e vales estão bem resolvidos (livre de oscilações), sendo que a solução numérica $\operatorname{com} \theta=0.2$ apresentou o melhor resultado.

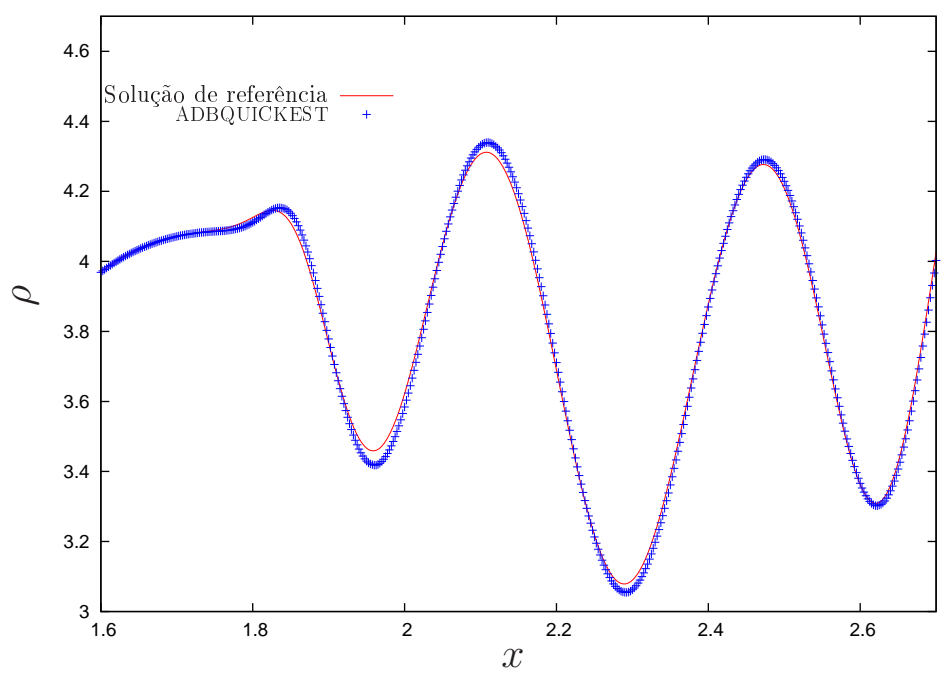

Figura 5.20: Ampliação das soluções numérica e de referência da Figura 5.18 na região [1.6, 2.7].

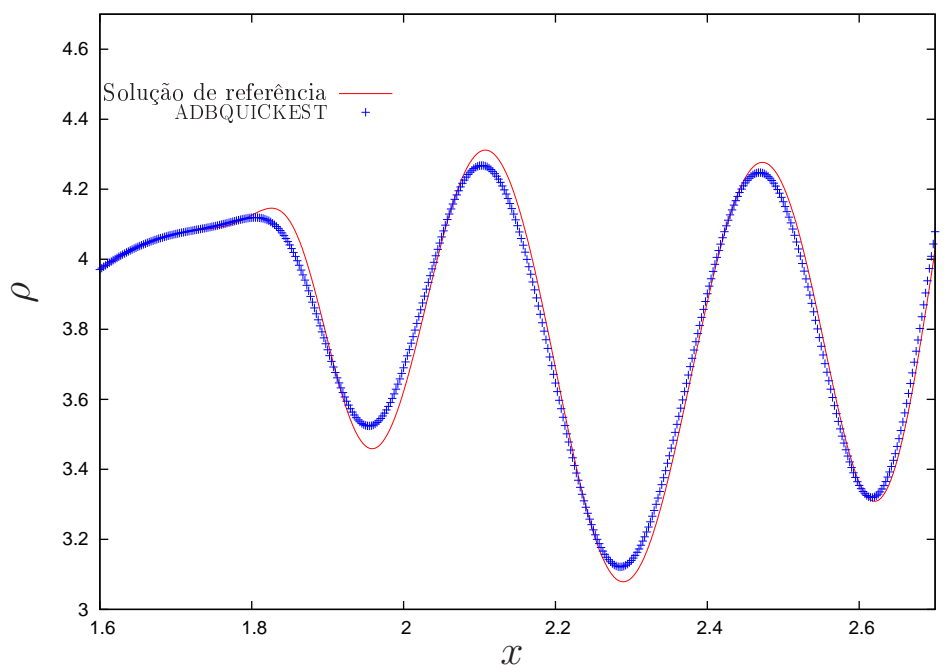

Figura 5.21: Ampliação das soluções numérica e de referência da Figura 5.19 na região [1.6, 2.7].

Problema teste 13: Este problema de Riemann, proposto por Titarev e Toro [139], é uma variação do tubo de choque de Shu-Osher e bastante difícil de se simular numericamente. Neste teste resolve-se o sistema de equações $(2.4)$ no domínio $[-5,5]$ com a condição inicial

$$
\mathbf{u}_{0}(x)= \begin{cases}(1.515695,0.523346,1.80500)^{T}, & x<-4.5 \\ (1+0.1 \sin (20 \pi x), 0,1)^{T}, & x \geq 4.5\end{cases}
$$


Para a simulação do problema, foram empregados os seguintes dados: duas malhas uniformes consistindo de 12500 e 18500 células computacionais, dois números de Courant, $\theta=0.2$ e $\theta=0.6$ e tempo final $t=5.0$. As Figuras 5.22 e 5.23 mostram a solução de referência (esquema "upwind" de 1ํㅡ ordem em uma malha de 25000 células) e os resultados numéricos computados com o esquema ADBQUICKEST para a densidade nas malhas 12500 e 18500 com $\theta=0.2$. As Figuras 5.24-5.27 mostram as ampliações das soluções numéricas e de referência nessas malhas para o caso em que $\theta=0.2$. Da mesma forma como feito para o caso em que $\theta=0.2$, mostram-se nas Figuras 5.28-5.33 as soluções e suas ampliações no caso em que $\theta=0.6$.

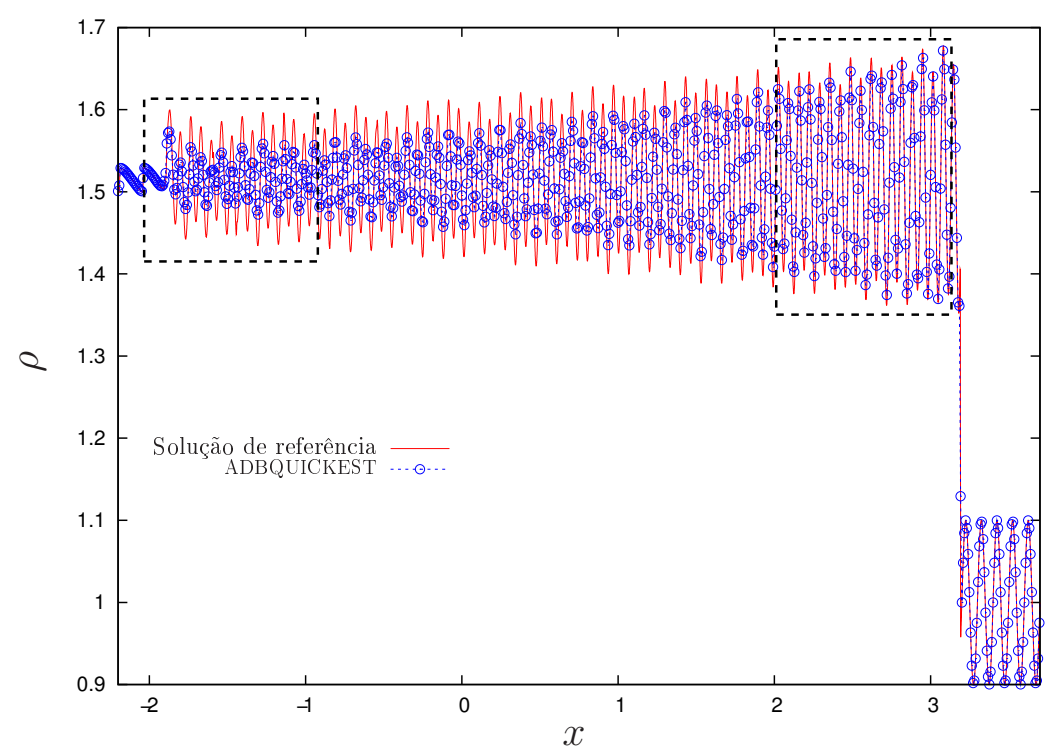

Figura 5.22: Perfil da densidade do problema teste $13 \operatorname{com} \theta=0.2$ e 12500 células.

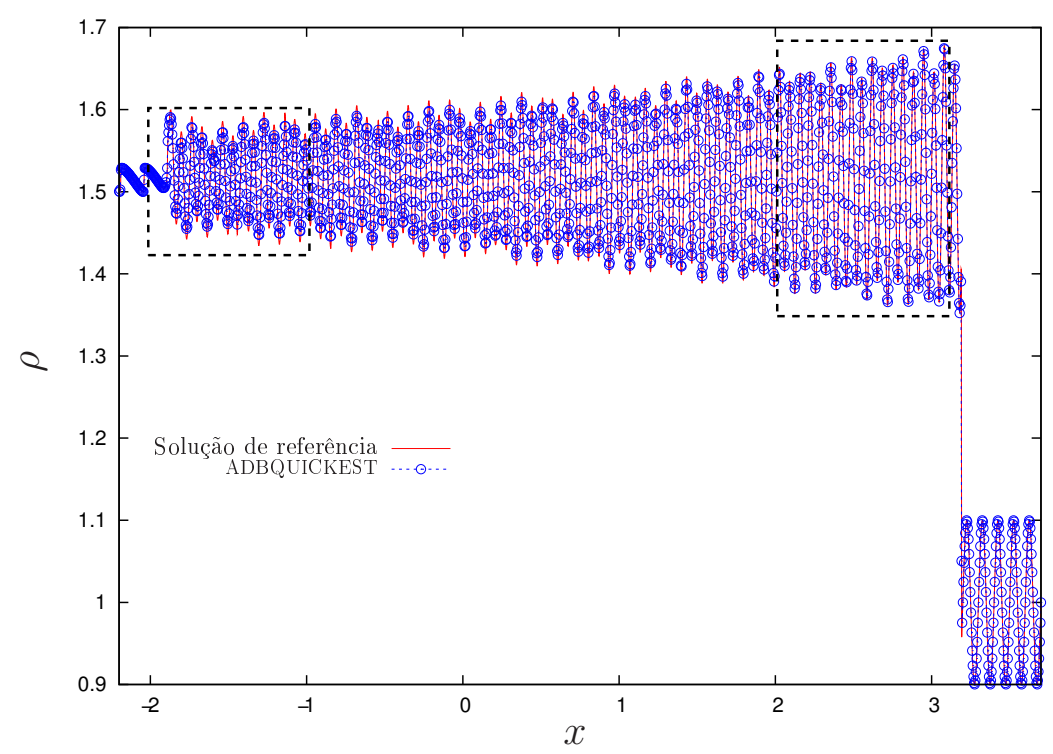

Figura 5.23: Perfil da densidade do problema teste $13 \operatorname{com} \theta=0.2$ e 18500 células. 


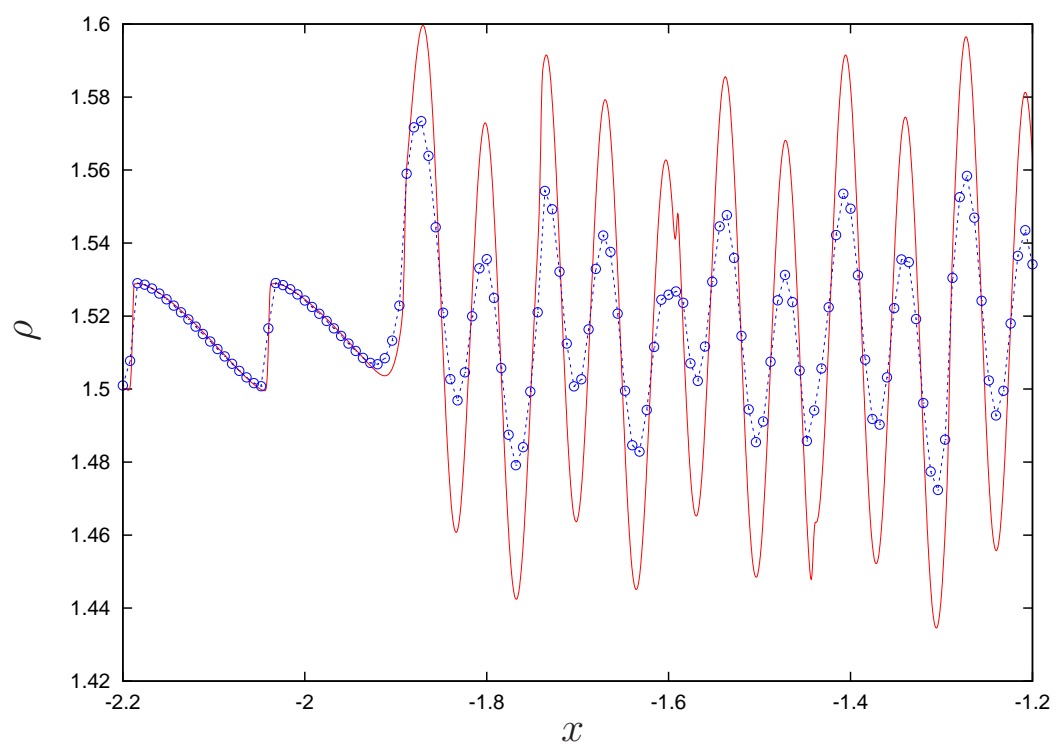

Figura 5.24: Ampliação das soluções numérica e de referência da Figura 5.22 na região $[-2.2,1.2]$.

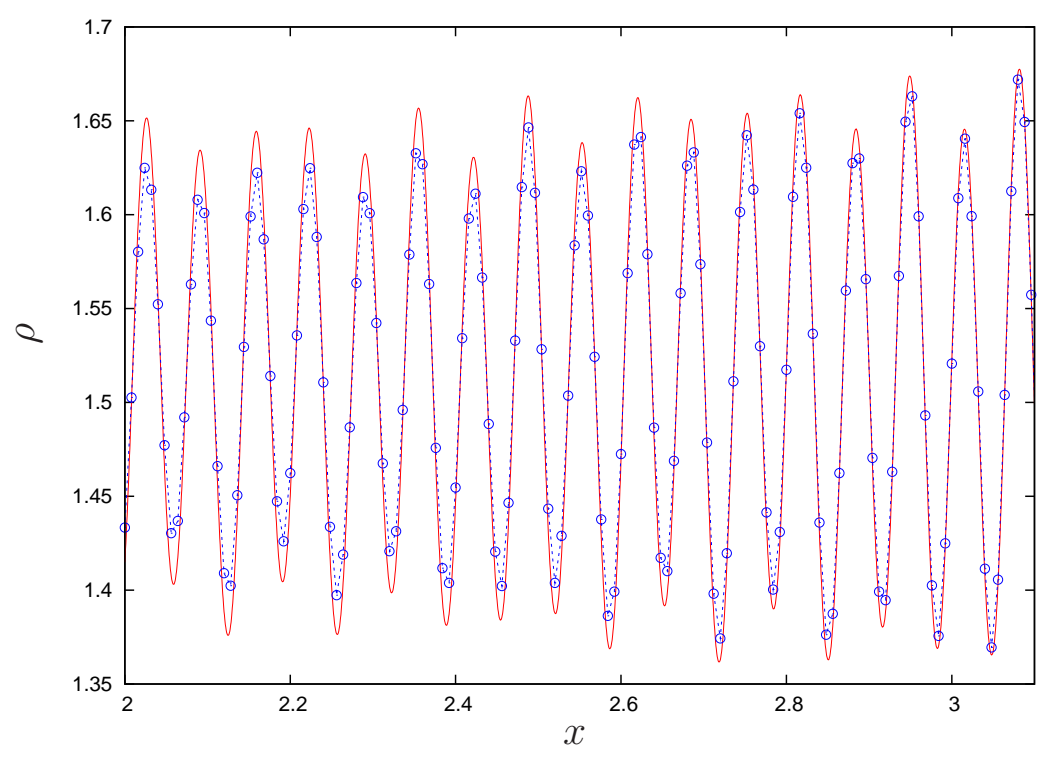

Figura 5.25: Ampliação das soluções numérica e de referência da Figura 5.22 na região [2.0, 3.2]. 


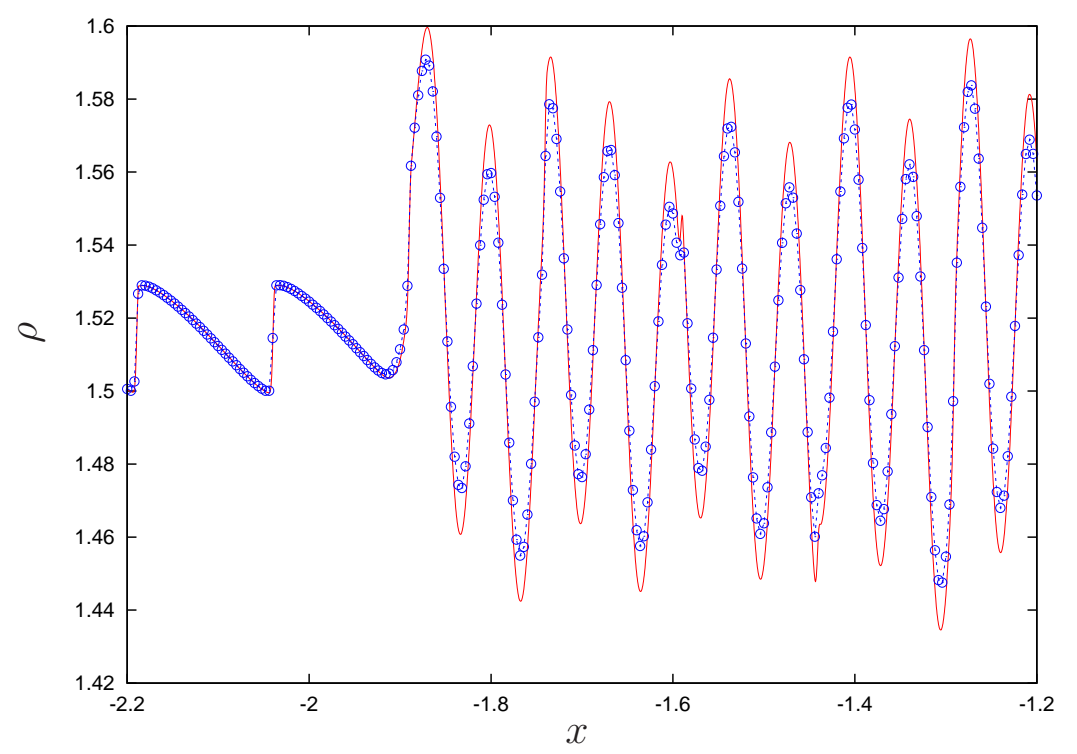

Figura 5.26: Ampliação das soluções numérica e de referência da Figura 5.23 na região $[-2.2,1.2]$.

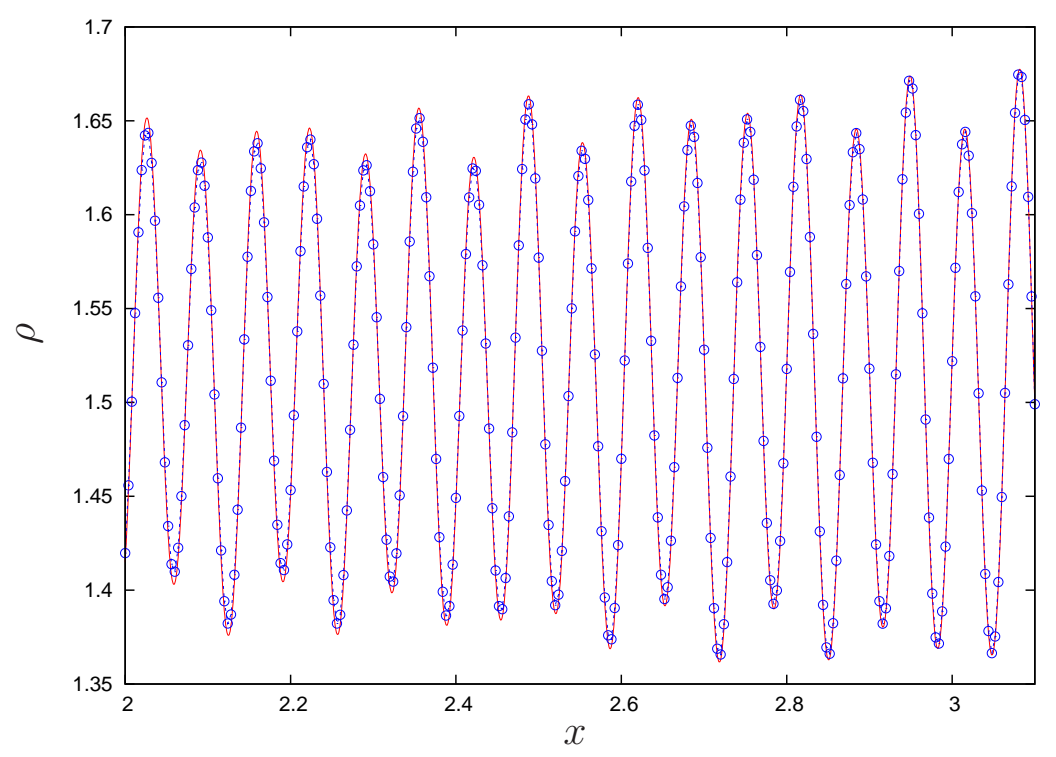

Figura 5.27: Ampliação das soluções numérica e de referência da Figura 5.23 na região [2.0, 3.2]. 


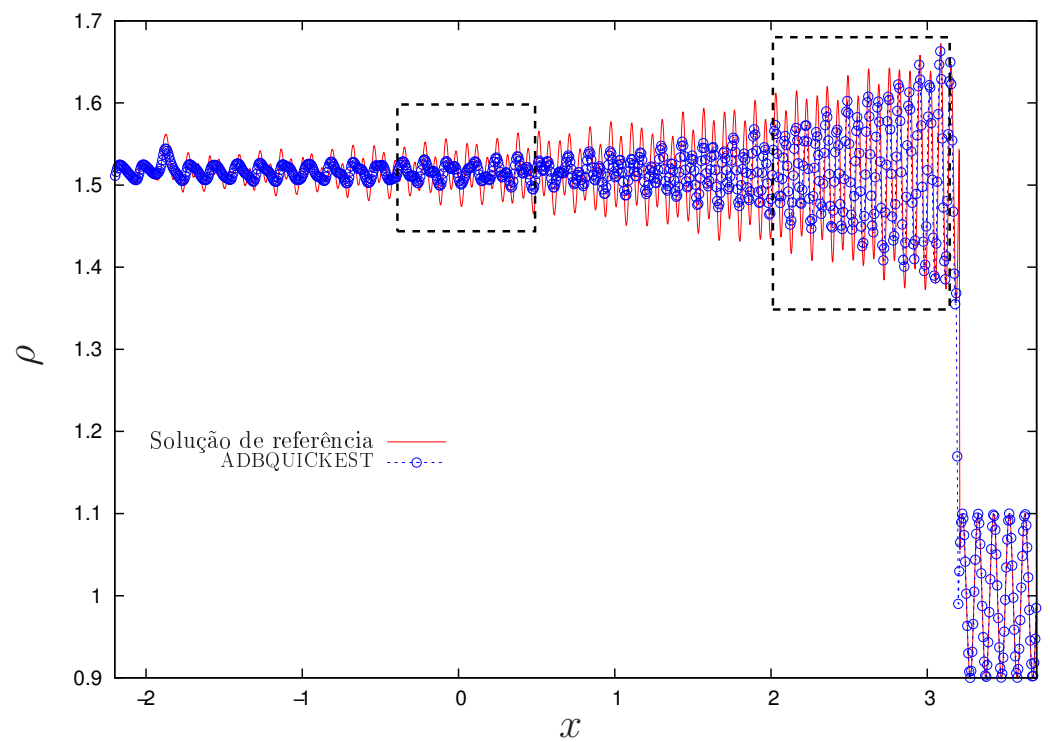

Figura 5.28: Perfil da densidade do problema teste $13 \operatorname{com} \theta=0.6$ e 12500 células.

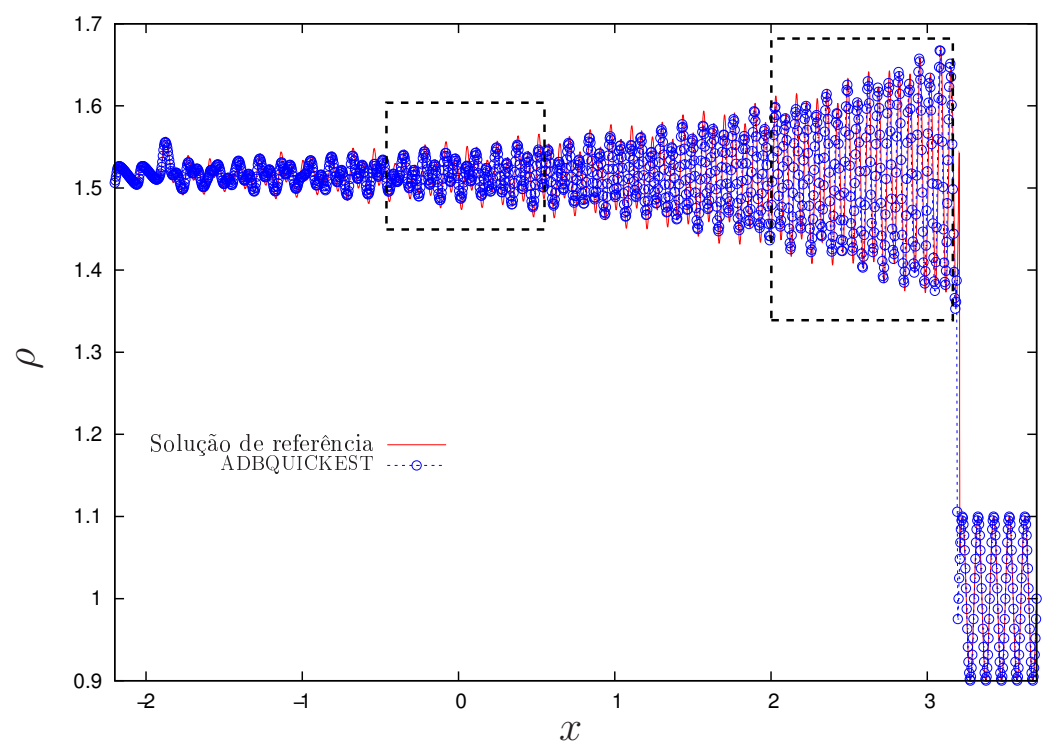

Figura 5.29: Perfil da densidade do problema teste $13 \operatorname{com} \theta=0.6$ e 18500 células. 


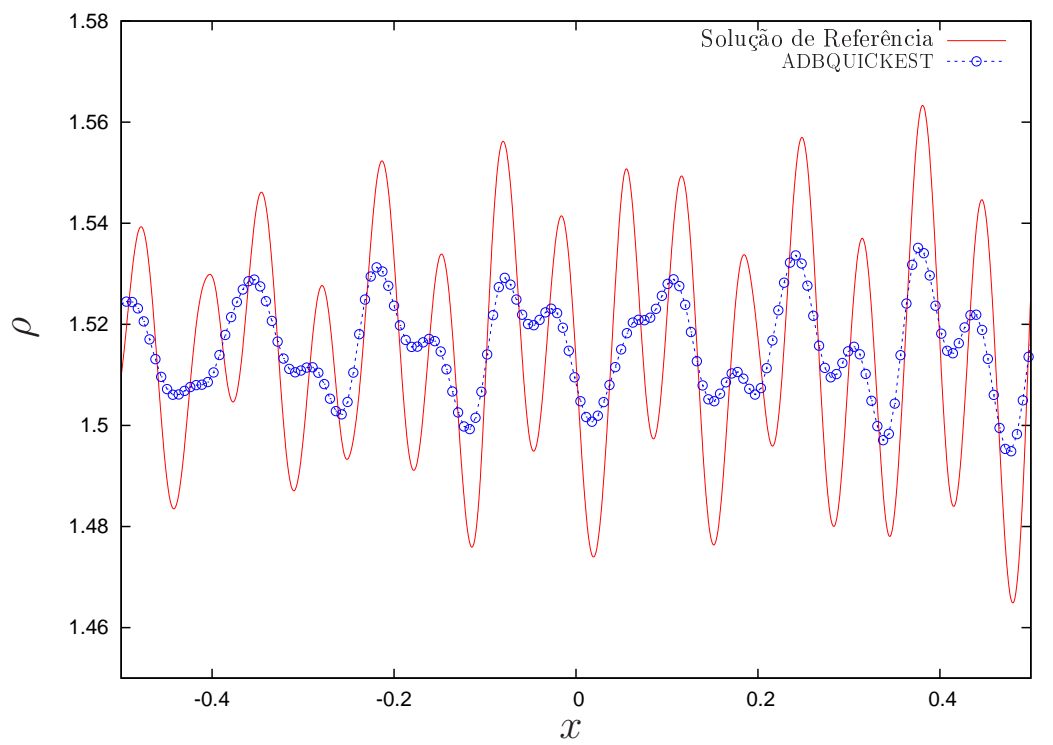

Figura 5.30: Ampliação das soluções numérica e de referência da Figura 5.28 na região $[-0.5,0.5]$.

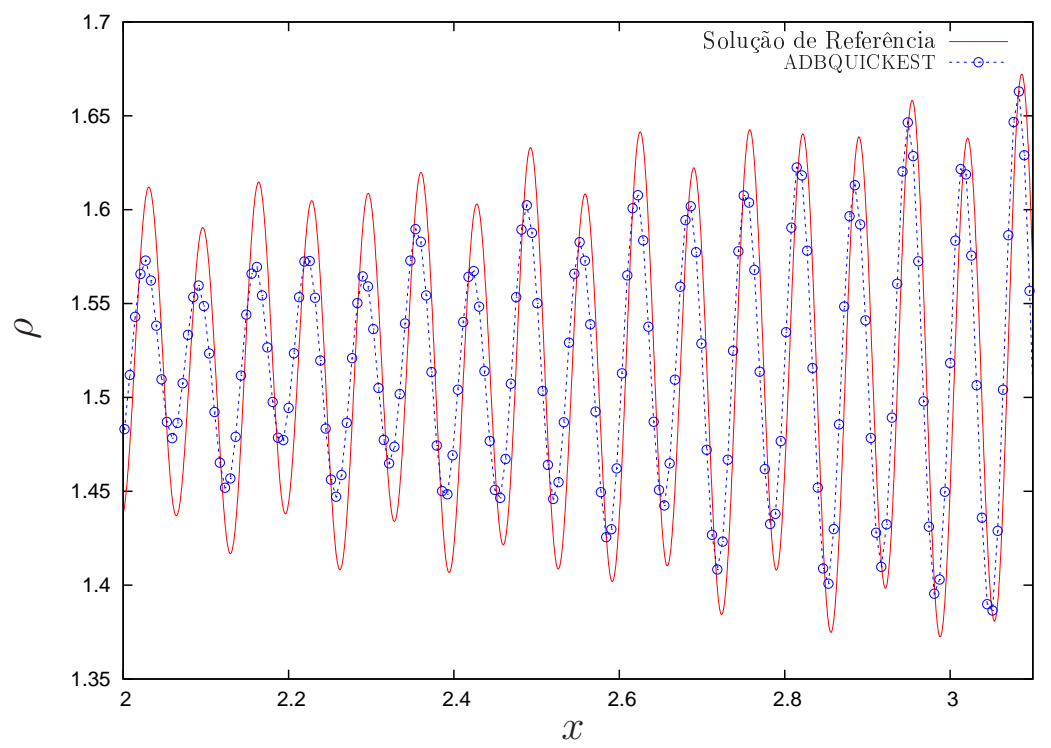

Figura 5.31: Ampliação das soluções numérica e de referência da Figura 5.28 na região [2.0, 3.2]. 


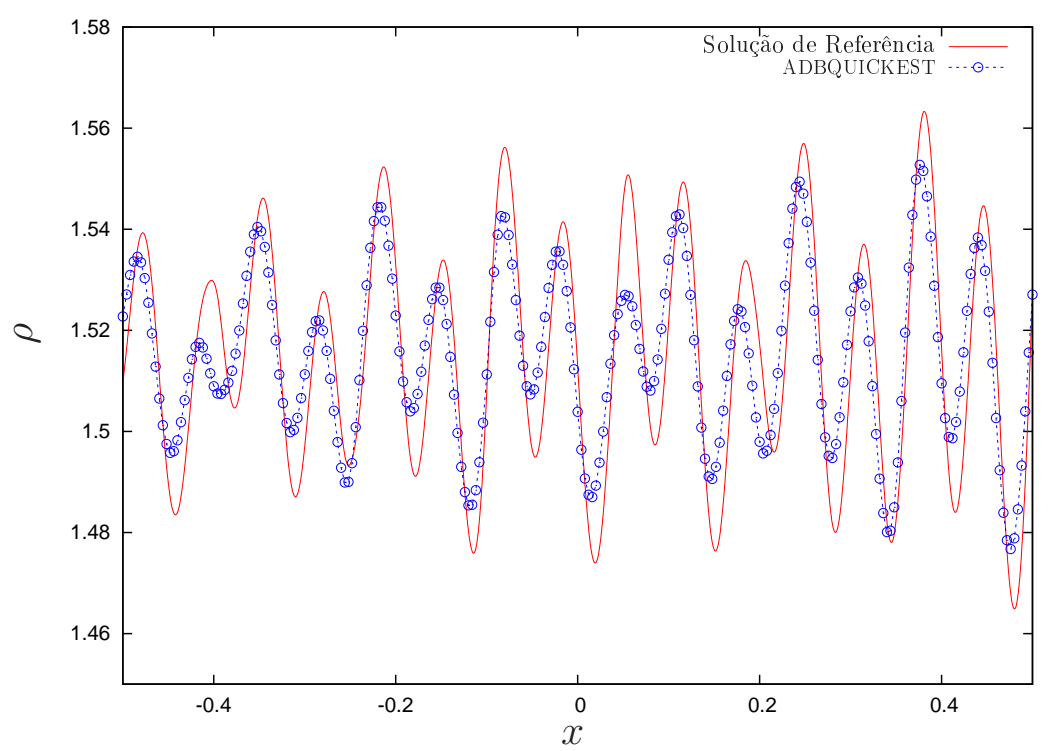

Figura 5.32: Ampliação das soluções numérica e de referência da Figura 5.29 na região $[-0.5,0.5]$.

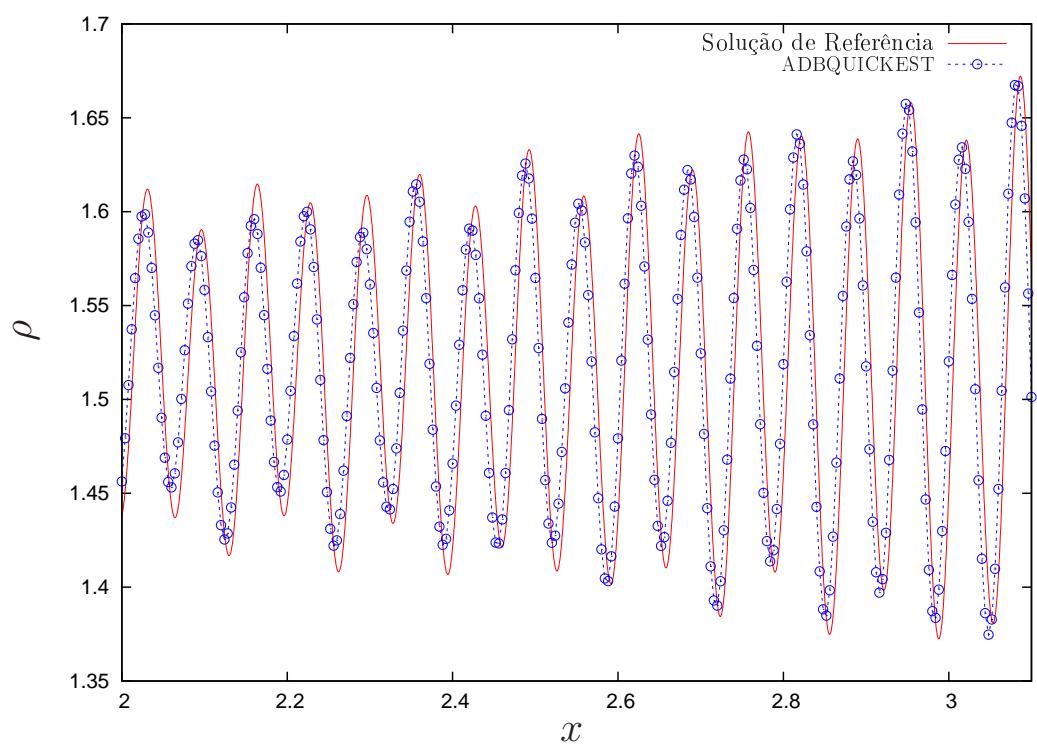

Figura 5.33: Ampliação das soluções numérica e de referência da Figura 5.29 na região [2.0, 3.2]. 
Para este problema teste não linear, pode-se ver por esses resultados apresentados que o esquema ADBQUICKEST na malha 18500 reproduz uma solução bastante próxima da solução de referência.

\subsubsection{Comentários}

Nesta seção o esquema ADBQUICKEST foi utilizado com sucesso para resolver uma variedade de problemas complexos de advecção-difusão 1D (advecção de escalares e Burgers) e problemas de Riemann 1D para as equações de Euler e águas rasas. De maneira geral, os resultados numéricos mostraram-se bastante satisfatórios, confirmando assim, a robustez e a capacidade desse esquema "upwind" de alta resolução em controlar oscilações não-físicas nas proximidades de descontinuidades com a introdução de pouca dissipação numérica. Os resultados referentes a essa pesquisa estão reunidos em um artigo publicado "online" no portal do periódico International Journal for Numerical Methods in Fluids (ver Ferreira et al. [41]). 


\subsection{Simulação de escoamentos laminares 2D e 3D}

Neste seção são apresentados os resultados numéricos de simulações de escoamentos laminares $2 \mathrm{D}$ e 3D utilizando-se o esquema "upwind" ADBQUICKEST implementado nos ambientes de simulações Freeflow 2D e 3D de Castelo et al. [19]. Em particular, seu desempenho é examinado na solução dos seguintes problemas: expansão brusca 2D ("backward facing step"), jato livre $2 \mathrm{D}$ sob à ação da gravidade incidindo perpendicularmente à uma superfície rígida impermeável e colapso de uma coluna de fluido em repouso nos casos 2D e 3D ("Broken Dam" ou "Dam Break").

\subsubsection{Expansão brusca 2D}

Um problema interessante em dinâmica dos fluidos e bem estudado ao longo dos anos é o escoamento laminar numa expansão brusca (Stuart e Dochan [130]), onde há uma recirculação principal causada pela súbita expansão. Esse problema de escoamento aparece em muitas aplicações de engenharia, como por exemplo, reatores, combustão de máquinas e dutos industriais. Ele foi escolhido aqui porque existe uma grande quantidade de dados, tanto numéricos como experimentais, para comparação.

O objetivo aqui é validar o código Freeflow $2 D$ atual, incrementado com o novo esquema ADBQUICKEST. Para isso, o comprimento da recirculação $x_{1}$ (ver Figura 5.59) é estimado e comparado com dados experimentais e numéricos. Este comprimento é determinado como sendo o ponto na primeira fila de células adjacentes ao contorno rígido inferior, em que a componente $u$ de velocidade troca de sinal. A geometria do problema está ilustrada na Figura 5.59, em que a altura da expansão brusca $h$ é metade da largura do canal. Para a simulação deste problema foram empregados diferentes números de Reynolds.

$\mathrm{Na}$ entrada do canal, o perfil de velocidade imposto é parabólico, isto é,

$$
u(y)=-\frac{4 U}{h^{2}}\left(y-\frac{h}{2}\right)^{2}+U
$$

em que $U$ é a escala de velocidade. O contorno de saída de fluido está localizado a $30 h$ do degrau. O número de Reynolds para esse problema é calculado por

$$
R e=\frac{2 h \bar{v}}{\nu}
$$

em que

$$
\bar{v}=\frac{1}{h} \int_{0}^{h} u(y) d y
$$

é a velocidade média na entrada do canal. 
Resolvendo a integral (5.16) e substituindo na equação (5.15), obtém-se

$$
R e=\frac{2}{3} U \frac{2 h}{\nu}
$$

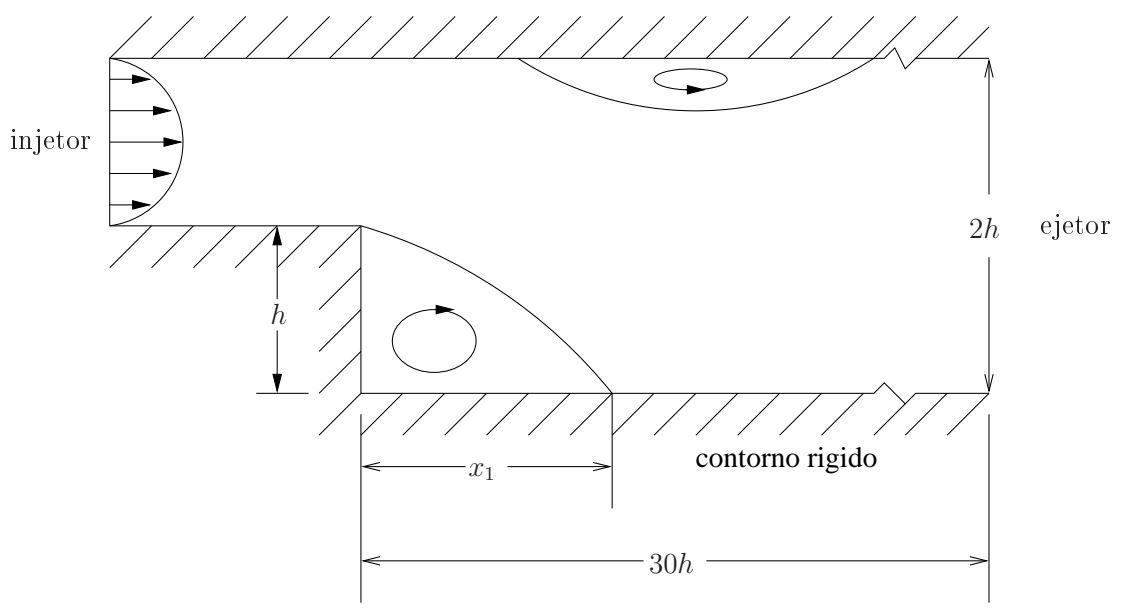

Figura 5.34: Geometria do problema da expansão brusca laminar.

Para a solução numérica do problema, são envolvidos dois números de Reynolds: o Reynolds numérico, $R e_{\text {num }}$, definido por

$$
R e_{\text {num }}=\frac{2 h U_{\max }}{\nu},
$$

na qual $U_{\max }=1.0 \mathrm{~ms}^{-1}$ é a velocidade máxima no perfil (5.14); e o Reynolds dado pela equação (5.17). Assim, comparando (5.17) e (5.18) obtêm-se a seguinte relação

$$
R e=\frac{2}{3} R e_{\text {num }}
$$

Os dados empregado para a simulação deste problema são:

- Dimensão do domínio: $4.0 \mathrm{~m} \times 0.2 \mathrm{~m}$;

- Entrada do injetor: $h=0.1 \mathrm{~m}$;

- Velocidade de injeção: $U_{\max }=1.0 \mathrm{~ms}^{-1}$;

- Parâmetros de escala: $2 h=0.2 \mathrm{~m}$ e $U_{\max }=1.0 \mathrm{~ms}^{-1}$;

Foram realizadas cinco simulações numa malha $400 \times 20$ células $(\delta x=\delta y=0.01 \mathrm{~m})$ utilizando-se os números de Reynolds 100, 200, 400, 600 e 800, que equivalem aos Reynolds numéricos 150, 300, 600, 900 e 1200, respectivamente. Essas simulações estão ilustradas nas Figuras 5.35-5.37 no tempo final 1000 s. Em particular, elas contêm os campos de pressão e as componentes $u$ e $v$ de velocidade, calculados usando o esquema ADBQUICKEST. Como pode-se observar nessas figuras, o comprimento da recirculação $x_{1}$ aumenta com o aumento do 
número de Reynolds. E, a partir do número de Reynolds $400\left(R e_{n u m}=600\right)$, pela Figura 5.36 (Campo de velocidade na direção $x$ ), nota-se o aparecimento de uma segunda recirculação no topo do canal. Isto está qualitativamente de acordo com os dados encontrados na literatura (ver as referências Kim e Moin [69], Stuart e Dochan [130], Liu e Chieng [87] e Wan et al. [152]).

\section{Campo de pressão}

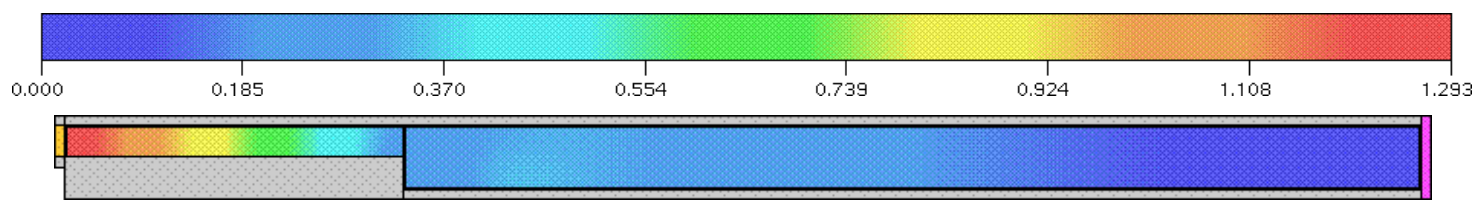

Campo de velocidade na direção $x$

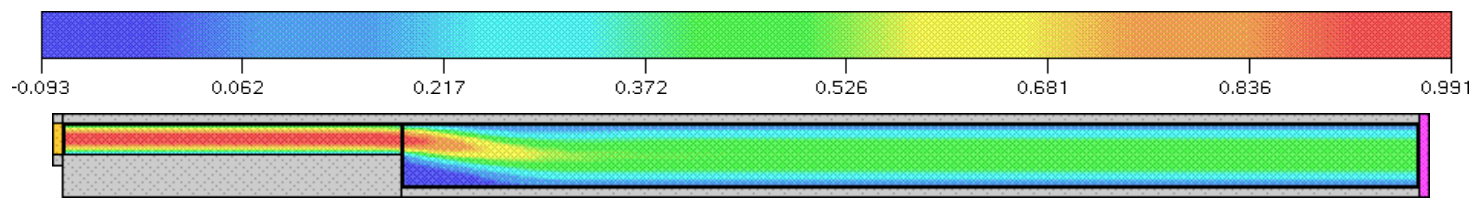

Campo de velocidade na direção $y$

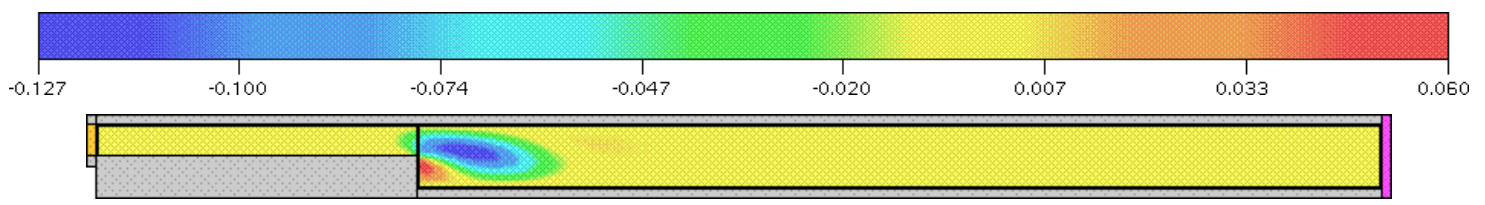

Figura 5.35: Problema da expansão brusca sem modelagem de turbulência para $R e=100$.

\section{Campo de pressão}

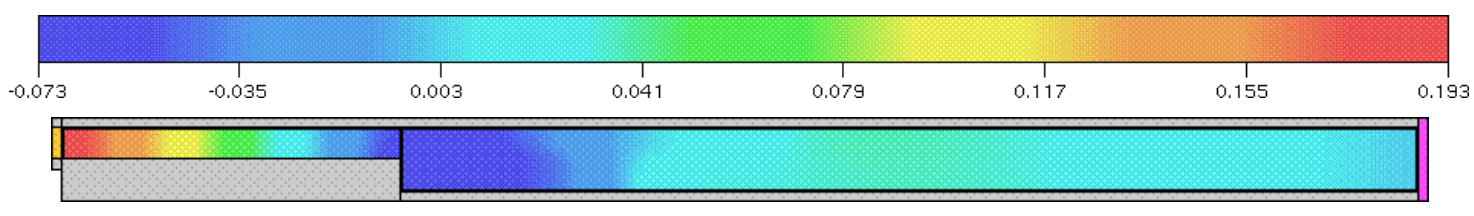

Campo de velocidade na direção $x$

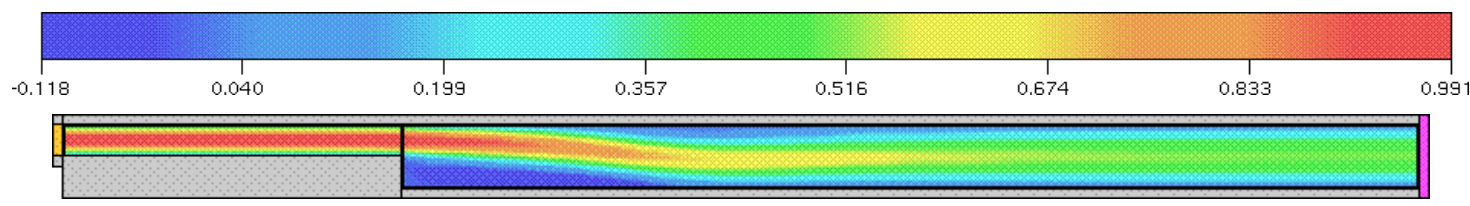

Campo de velocidade na direção $y$

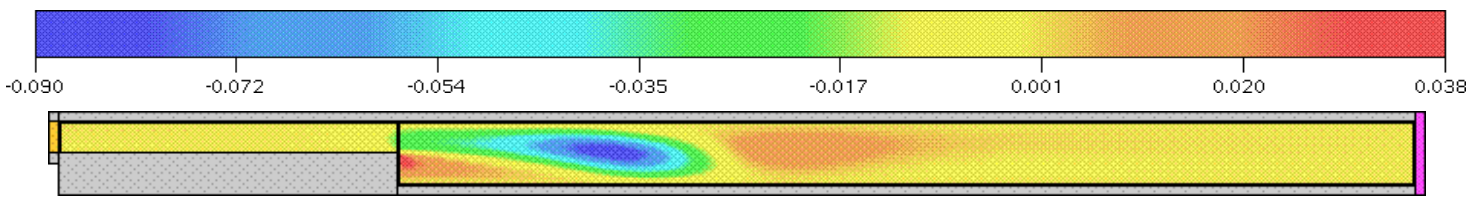

Figura 5.36: Problema da expansão brusca sem modelagem de turbulenta para $R e=400$. 


\section{Campo de pressão}

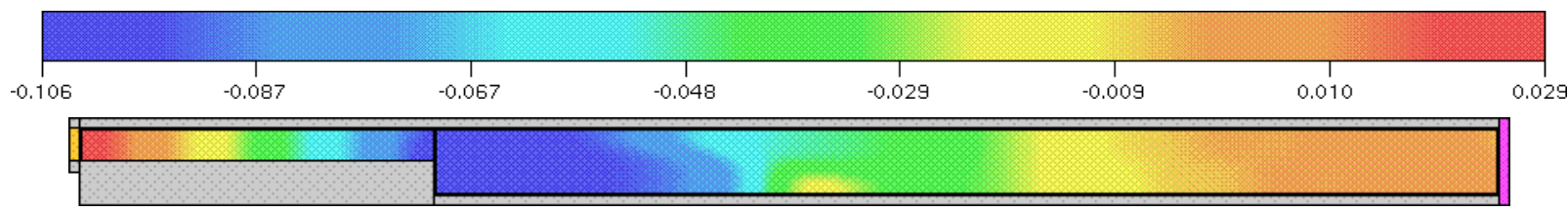

Campo de velocidade na direção $x$

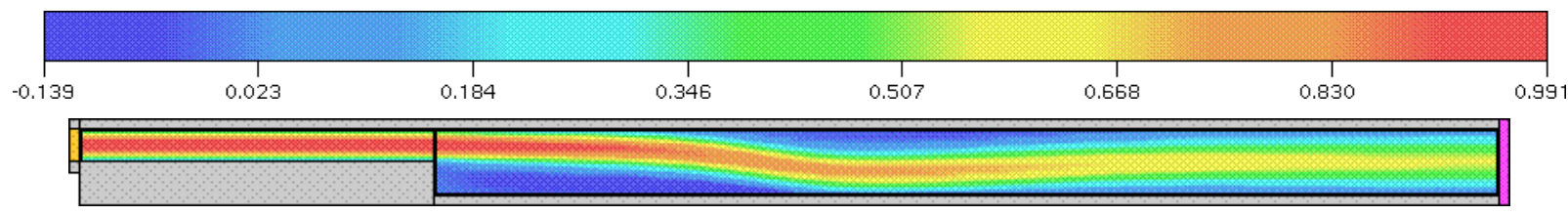

Campo de velocidade na direção $y$

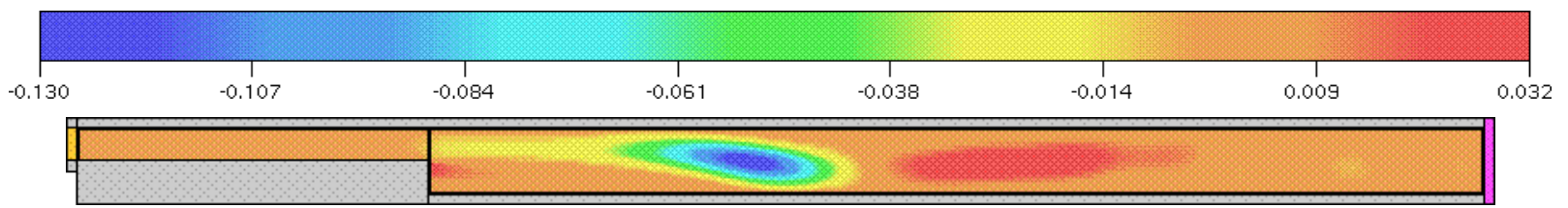

Figura 5.37: Problema da expansão brusca sem modelagem de turbulenta para $R e=800$.

Tabela 5.3: Valores do comprimento da recirculação $x_{1}$ dos dados experimentais e numéricos para diferentes números de Reynolds.

\begin{tabular}{|c|c|c|c|c|}
\hline \multirow{2}{*}{$R e$} & Experimental & \multicolumn{3}{|c|}{ Numérico } \\
\cline { 2 - 5 } & Armaly & Armaly & Stuart & ADBQUICKEST \\
\hline \hline 100 & 3.06 & 2.95 & 2.86 & 3.08 \\
\hline 200 & 5.16 & 4.82 & 4.97 & 5.14 \\
\hline 400 & 8.72 & 8.04 & 8.03 & 8.24 \\
\hline 600 & 11.28 & 8.18 & 10.25 & 9.71 \\
\hline 800 & 14.34 & 7.50 & 11.47 & 11.68 \\
\hline
\end{tabular}

A Tabela 5.3 mostra estimativas, tanto as experimentais de Armaly et al. [6] quanto as numéricas de Stuart e Dochan [130] mais aquelas obtidas usando o ADBQUICKEST, para o comprimento da recirculação $x_{1}$ a diferentes números de Reynolds $(100 \leq R e \leq 800)$. Observa-se nessa tabela que, os resultados obtidos com o ADBQUICKEST, para baixos números de Reynolds $(R e \leq 400)$, estão em boa concordância com os dados experimentais de Armaly et al. Por outro lado, para $R e>400$, os resultados numéricos não são tão bons. Essa diferença pode ser atribuída aos efeitos 3D e, possivelmente, ao efeito de transição para turbulência no problema. Por essa mesma tabela, vê-se também, que no geral os resultados com ADBQUICKEST 
são melhores que os numéricos de Armaly et al. e compatíveis com os resultados de Stuart e Dochan. Em particular, para uma melhor visualização dos resultados, os dados da Tabela 5.3 são apresentados na Figura 5.38, que ilustra graficamente os valores do comprimento da recirculação $x_{1}$ contra o com o Reynolds.

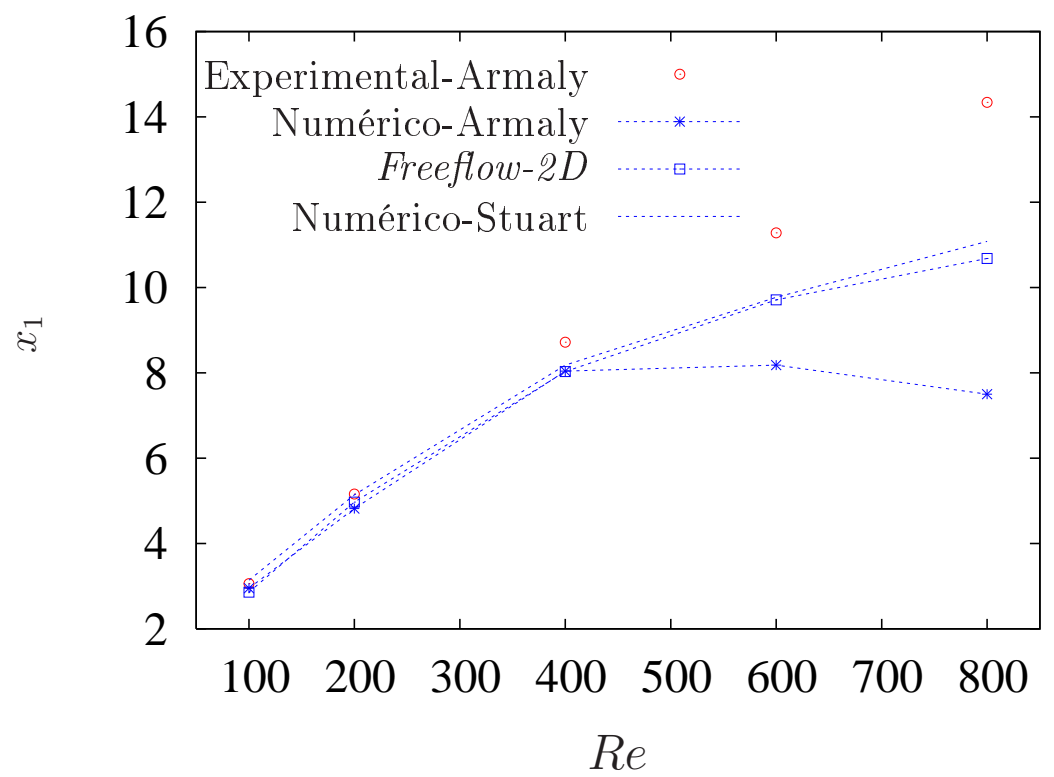

Figura 5.38: Comparação dos resultados experimentais e numéricos usando o esquema ADBQUICKEST.

Além disso, um teste de convergência do método numérico foi realizado com número de Reynolds experimental 266 e usando quatro malhas uniformes consistindo de $175 \times 10$ células $(\delta x=\delta y=0.02 \mathrm{~m}), 350 \times 20$ células $(350 \times 20$ células, $\delta x=\delta y=0.01 \mathrm{~m}), 700 \times 40$ células $(\delta x=\delta y=0.005 \mathrm{~m})$ e $875 \times 50$ células $(\delta x=\delta y=0.004 \mathrm{~m})$. Para esse teste, foram utilizados os seguintes dados:

- Dimensão do domínio: $3.5 \mathrm{~m} \times 0.2 \mathrm{~m}$;

- Entrada do injetor: $h=0.1 \mathrm{~m}$;

- Velocidade de injeção: $U_{\max }=1.0 \mathrm{~ms}^{-1}$;

- Coeficiente de viscosidade cinemática: $\nu \approx 0.00075 \mathrm{~m}^{2} \mathrm{~s}^{-1}$;

- Parâmetros de escala: $2 h=0.2 \mathrm{~m}$ e $U_{\max }=1.0 \mathrm{~ms}^{-1}$;

- Número de Reynolds: $R e=266$.

A Figura 5.39 mostra os perfis de velocidade $u$ adjacentes ao contorno rígido inferior, nas quatro malhas. A partir dessa figura, pode-se notar que, a medida que se refina a malha, a interseç̧ão desses perfis com a reta $y=0$ (posição onde a componente $u$ troca de sinal) converge para um valor próximo de 1.632. Assim, o valor estimado para o comprimento $x_{1}$, que neste caso, é em torno de 6.32, está bastante próximo do experimental (ver Tabela 5.3). 


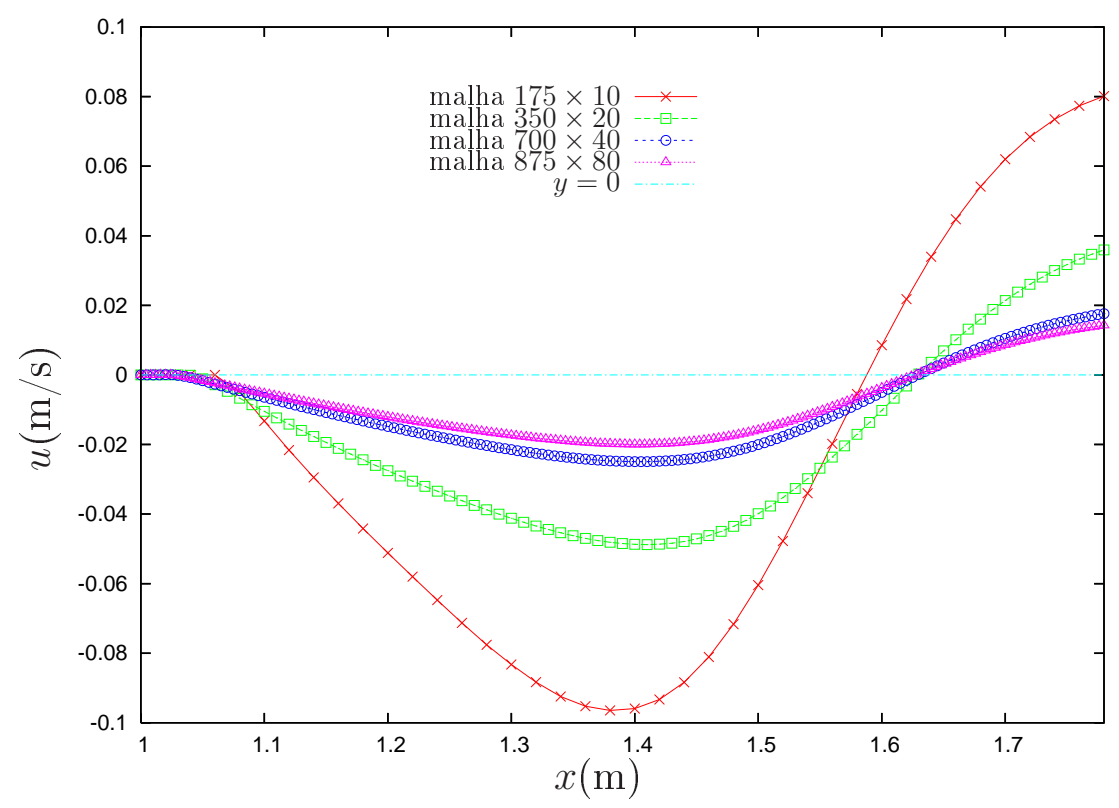

Figura 5.39: Perfis da componente $u$ da velocidade do problema da expansão brusca, mostrando a convergência da solução numérica nas quatro malhas usando o esquema ADBQUICKEST.

\subsubsection{Jato livre 2D sobre uma superfície rígida impermeável}

O problema 2D considerado agora foi escolhido para a verificação do código Freeflow $2 D$ incrementado com o novo esquema ADBQUICKEST. O problema em questão é o escoamento de um jato livre, em regime laminar, incidindo perpendicularmente a uma superfície rígida impermeável e sob o efeito do campo gravitacional. Este problema com superfície livre móvel tem solução analítica, dada por Watson [155], para a altura entre a superfície rígida e a superfície livre do fluido (ver Figura 5.40). A seguir é apresentada a solução analítica de Watson nesse caso.

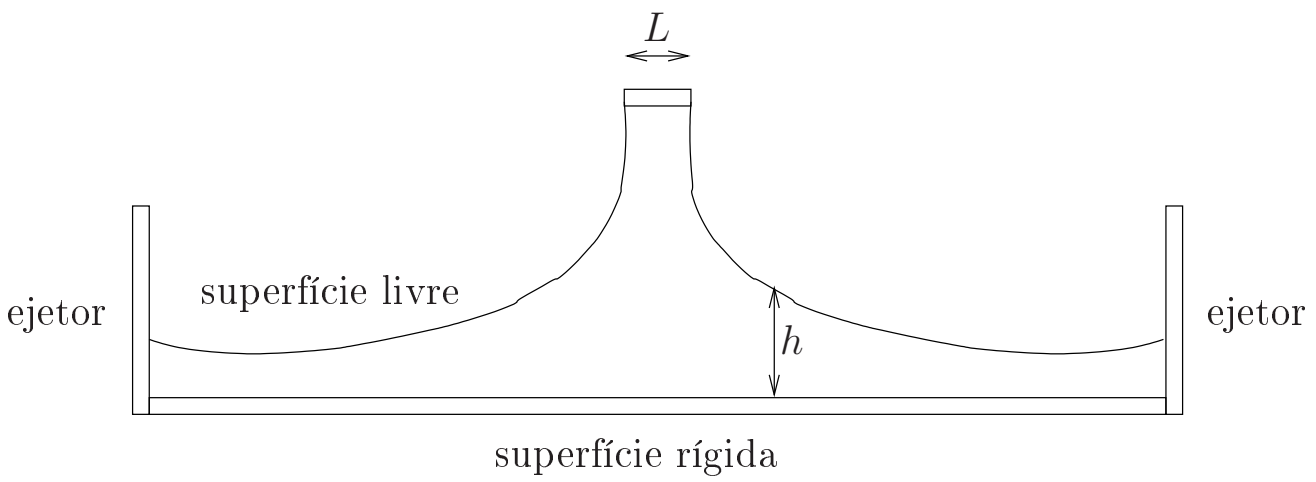

Figura 5.40: Ilustração da superfície livre de um jato de fluido incidindo sobre uma superfície rígida.

Desprezando os efeitos de tensão superficial, após o escoamento atingir o estado estacionário, Watson deduziu a seguinte relação explícita para a altura $h$ entre a superfície rígida e a superfície 
livre do fluido

$$
h(x)=\left\{\begin{array}{lll}
\frac{\pi}{\sqrt{3}} \frac{\nu(x+l)}{Q}, & \text { se } & x \geq x_{0} \\
a+\left(1-2 \pi / 3 \sqrt{3} c^{2}\right) \delta, & \text { se } & x<x_{0}
\end{array}\right.
$$

em que

$$
\begin{aligned}
x_{0} & =\left(\frac{3 \sqrt{3}(1.402)(\pi-(1.402) \sqrt{3})}{2 \pi^{2}}\right) a R e \\
l & =\left(\frac{3 \sqrt{3}(1.402)(2 \sqrt{3}(1.402)-\pi)}{2 \pi^{2}}\right) a R e
\end{aligned}
$$

$\mathrm{e}$

$$
\delta^{2}(x)=\frac{3 \sqrt{3} c^{3}}{2(\pi-c \sqrt{3})} \frac{\nu x}{U},
$$

na qual $c$ é uma constante dada por $c=1.402$ e $U$ a velocidade de injeção.

Na equação (5.20) $Q$ é a vazão definida por $Q=a U$, em que $a=L / 2$. Mais detalhes sobre a descrição da solução analítica de Watson podem ser encontrados no próprio artigo de Watson [155] ou em Ferreira et al. [44]. Para esta simulação deste problema, o seguinte modelo foi empregado:

- Dimensão do domínio: $0.5 \mathrm{~m} \times 0.05 \mathrm{~m}$;

- Diâmetro do injetor: $L=0.01 \mathrm{~m}$;

- Velocidade de injeção: $U=1.0 \mathrm{~ms}^{-1}$;

- Constante gravitacional: $g=9.81 \mathrm{~ms}^{-2}$;

- Coeficiente de viscosidade cinemática: $\nu=5.0 \times 10^{-6} \mathrm{~m}^{2} \mathrm{~s}^{-1}$;

- Parâmetros de escala: $L=0.01 \mathrm{~m}$ e $U=1.0 \mathrm{~ms}^{-1}$;

- Altura do injetor a partir da superfície rígida: $H=0.047 \mathrm{~m}$;

- Número de Reynolds: $R e=U L / \nu=2000$;

- Número de Froude: $F r=U / \sqrt{g L}=3.19275$.

O domínio utilizado para essa simulação está ilustrado na Figura 5.41.

As Figuras 5.42 e 5.43 mostram, além da evolução da superfície livre, os contornos de pressão e de velocidade $u$, respectivamente, na malha $500 \times 50$ células computacionais. A Figura 5.44 (e sua ampliação mostrada na Figura 5.45) mostra no tempo $t=5.0 \mathrm{~s}$ a comparação entre a solução analítica de Watson e a superfície livre calculada nas quatro malhas $100 \times 10$ células computacionais $(\delta x=\delta y=0.005 \mathrm{~m}), 250 \times 25$ células computacionais $(\delta x=\delta y=0.002 \mathrm{~m})$, $500 \times 50$ células computacionais $(\delta x=\delta y=0.001 \mathrm{~m})$ e $1000 \times 100$ células computacionais $(\delta x=\delta y=0.0005 \mathrm{~m})$. 


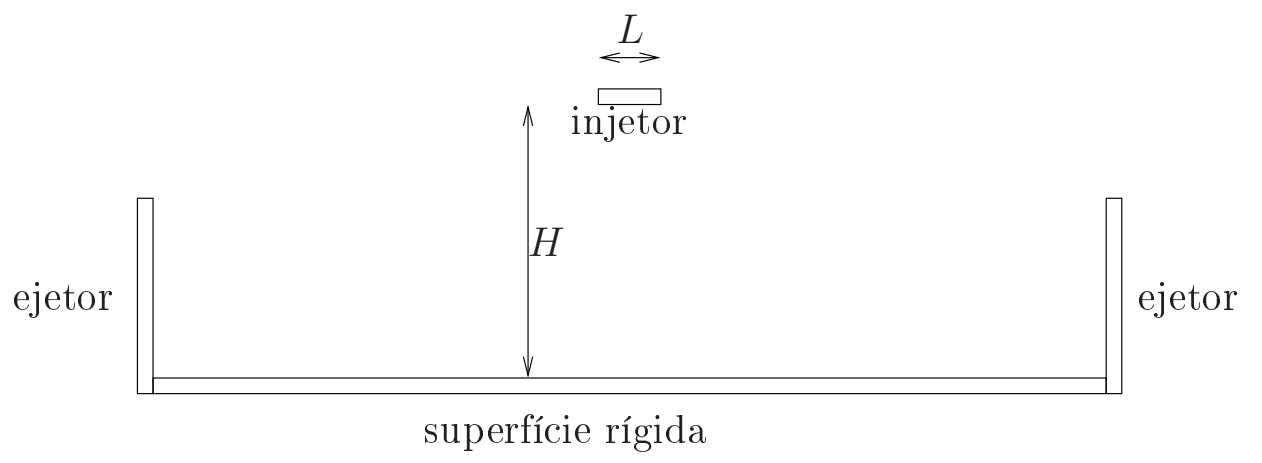

Figura 5.41: Domínio do problema do jato livre sobre uma superfície rígida.

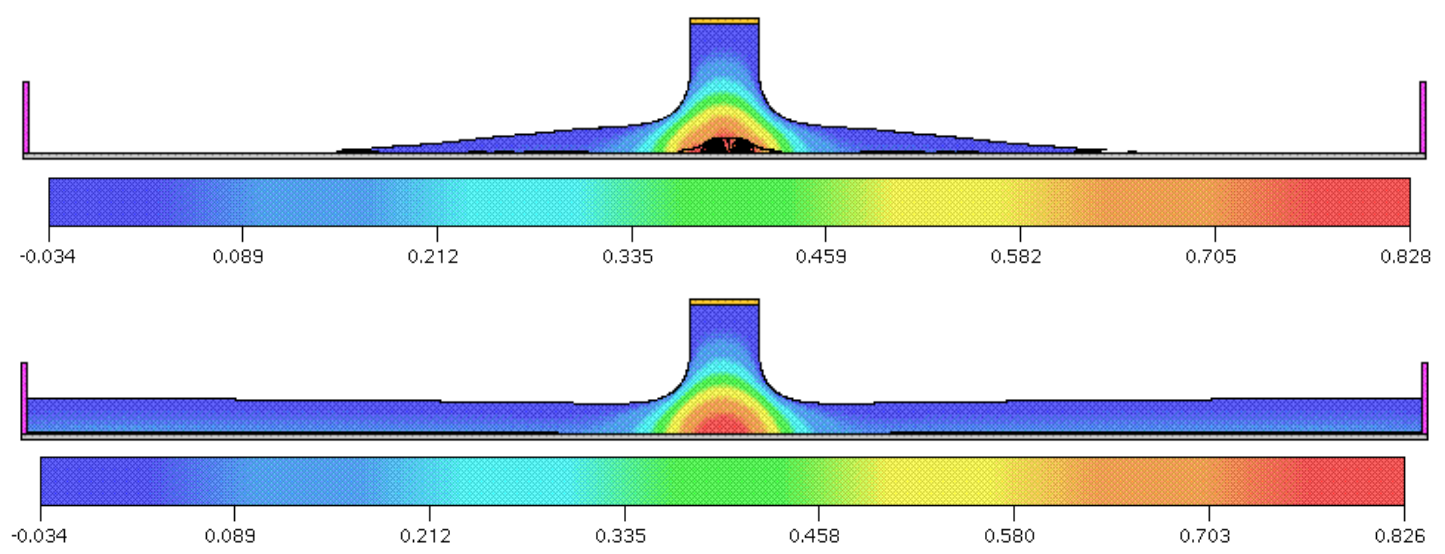

Figura 5.42: Evolução do perfil da superfície livre e contorno da pressão para o problema do jato livre no tempo $t=5.0 \mathrm{~s}$.

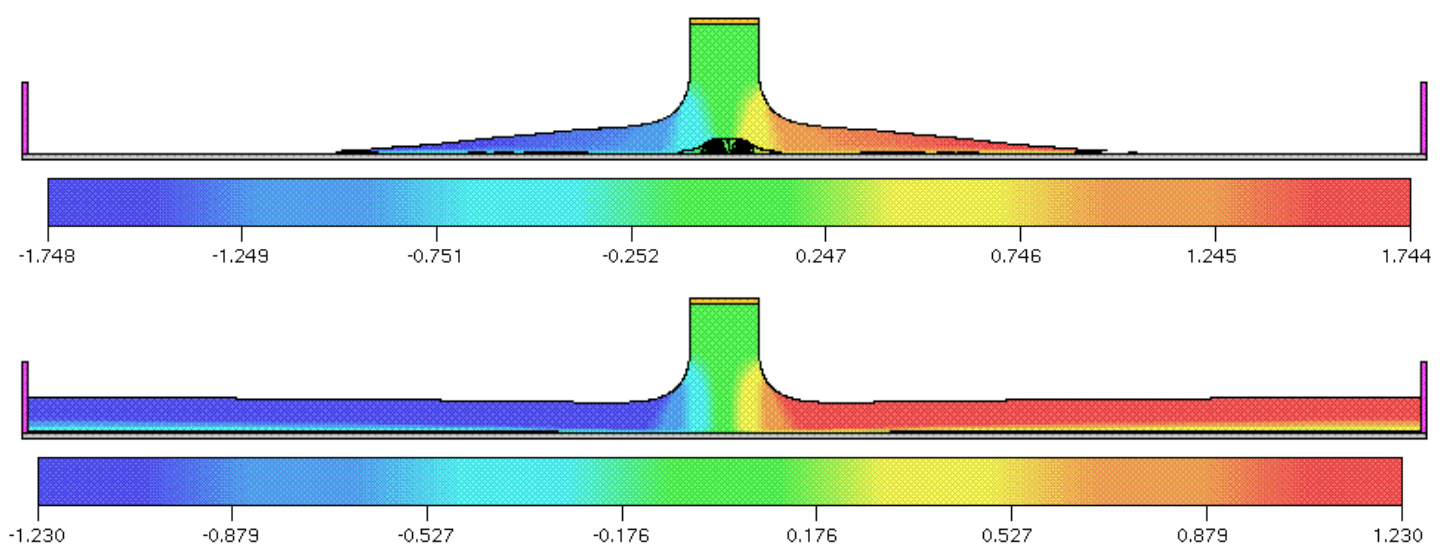

Figura 5.43: Evolução do perfil da superfície livre e contorno da velocidade na direção $x$ para o problema do jato livre no tempo $t=5.0 \mathrm{~s}$. 


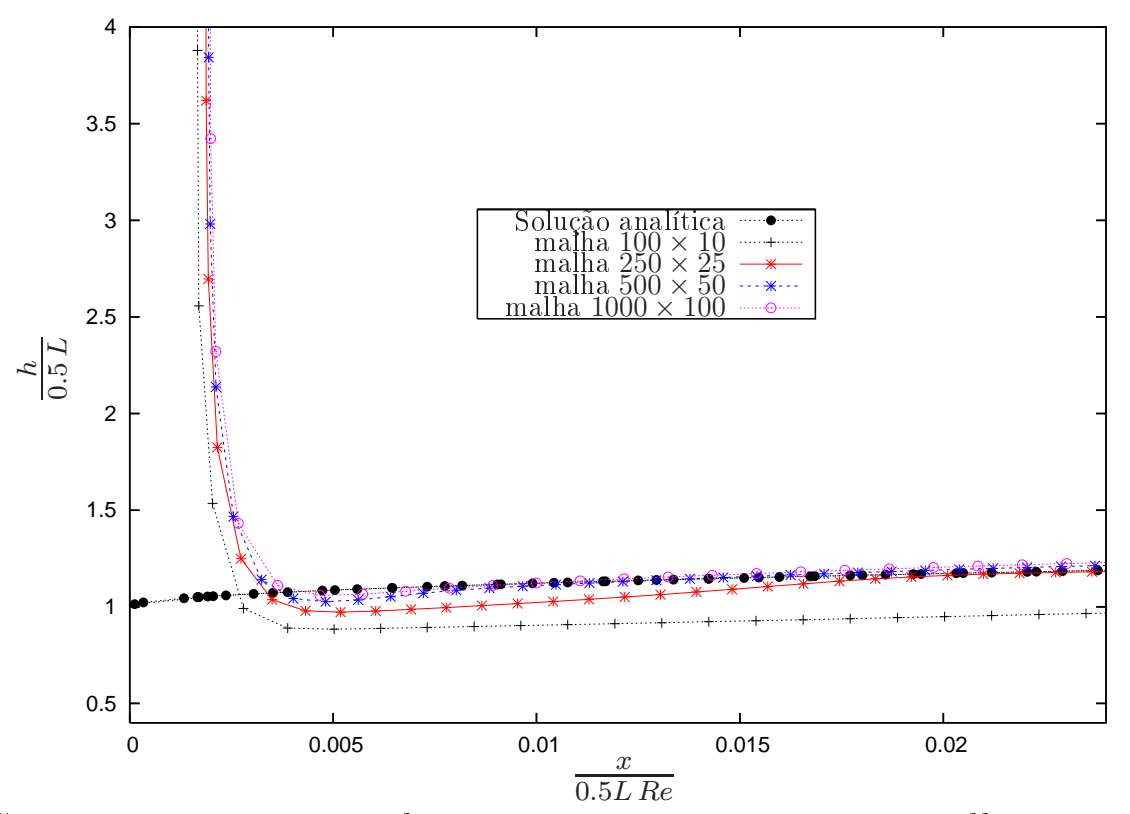

Figura 5.44: Comparação entre as soluções numéricas nas quatro malhas utilizando o esquema ADBQUICKEST e a solução analítica de Watson.

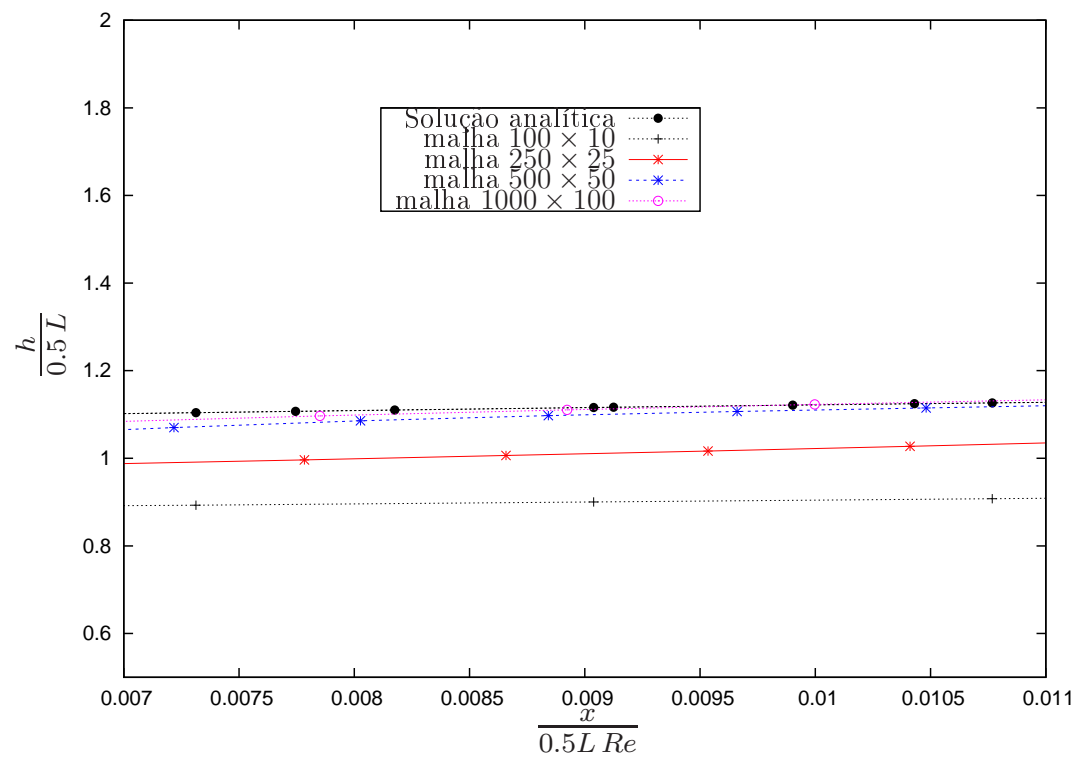

Figura 5.45: Ampliação das soluções numéricas e da solução analítica de Watson.

Pode-se observar a partir dessa figura que os resultados numéricos nas malhas mais finas estão em boa concordância com a solução analítica, porém, indicando pequenas diferenças em algumas regiões. Essas diferenças podem ser atribuídas ao número insuficiente de pontos da malha junto à parede rígida. Além disso, pode ser visto a partir dessa mesma figura que a solução numérica converge monotonicamente para uma solução muito próxima da solução analítica. A convergência pode ser melhor visualizada na ampliação das soluções numéricas e da solução analítica de Watson na região [0.007, 0.011] ilustrada na Figura 5.45. 


\subsubsection{Colapso de fluido: casos 2D e 3D}

A validação dos resultados numéricos com o esquema ADBQUICKEST são realizados aqui no problema do colapso de uma coluna de fluido nos casos 2D e 3D. Este tipo de escoamento com superfície livre móvel é conhecido na literatura como "Broken Dam" ou "Dam-Break". Esse problema é clássico em engenharia civil e útil para o entendimento do escoamento de água em barragens (Ataie-Ashtiani Farhadi [8]). O problema foi estudado experimentalmente no passado por Martin e Moyce [93] com o objetivo de investigar o espalhamento horizontal do fluido (no caso água) e a taxa de decaimento (altura) da coluna de fluido com o tempo. Mais tarde, Koshizuka e Oka [70] repetiram o experimento de Martin e Moyce para validar um código semi-implícito de simulação de escoamentos. Desde então, o problema tem sido investigado numericamente por vários pesquisadores (ver, por exemplo, Greaves [49]), como um teste de validação de métodos numéricos para escoamentos com superfícies livres móveis.

Para a simulação deste problema, foi considerada uma coluna de fluido retangular com $a=0.05 \mathrm{~m}$ de largura e $b=0.1 \mathrm{~m}$ de altura (no caso 3D, um bloco de $a=0.05 \mathrm{~m}$ de comprimento, $b=2 a$ largura e $c=2 a$ de altura) em equilíbrio hidrostático confinada entre paredes fixas.

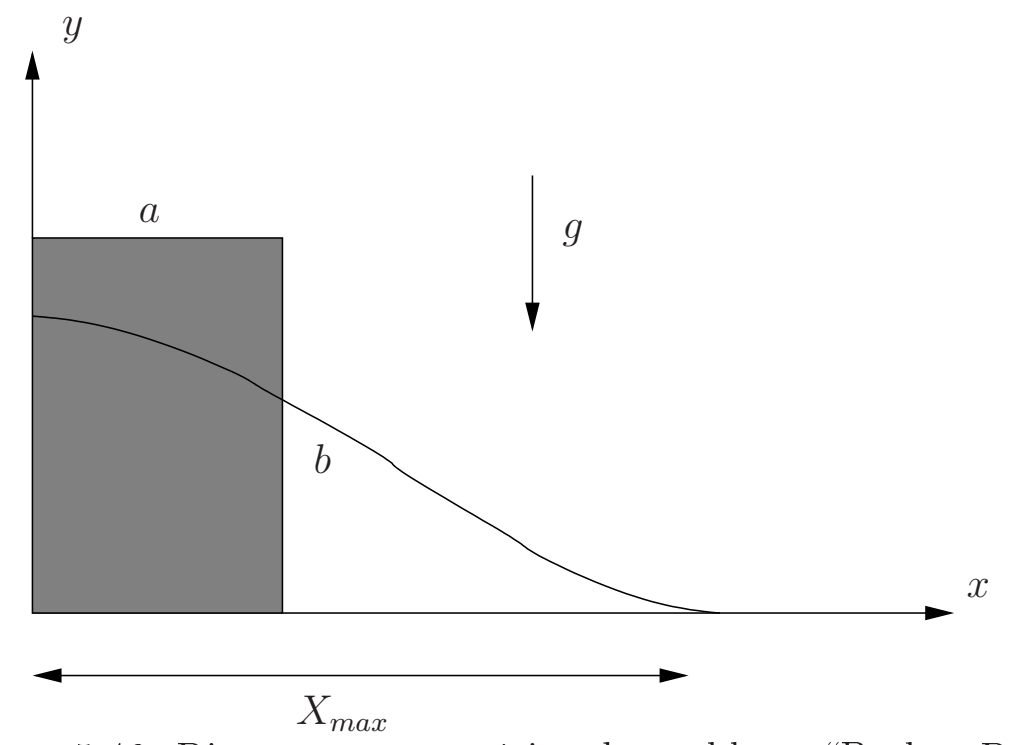

Figura 5.46: Diagrama esquemático do problema "Broken Dam".

A Figura 5.46 descreve a geometria do problema no caso 2D. No tempo $t=0 \mathrm{~s}$, a coluna de fluido é instantaneamente posta à ação da gravidade e, consequentemente, sofrendo um colapso deslizando-se na direção horizontal. A fim de comparar os resultados numéricos com os dados experimentais, a condição "free-slip" foi utilizada nas paredes.

Para a simulação numérica do problema, foram utilizadas as malhas $150 \times 75(\delta x=\delta y=$ $0.002 \mathrm{~m})$ células, $300 \times 150(\delta x=\delta y=0.001 \mathrm{~m})$ células e $600 \times 300(\delta x=\delta y=0.0005 \mathrm{~m})$ células no caso $2 \mathrm{D}$ e, $150 \times 50 \times 75(\delta x=\delta y=0.002 \mathrm{~m})$ no caso $3 \mathrm{D}$, mais os seguintes dados: 
- Dimensão do domínio 2D: $0.3 \mathrm{~m} \times 0.15 \mathrm{~m}$;

- Dimensão do domínio 3D: $0.3 \mathrm{~m} \times 0.10 \times 0.15 \mathrm{~m}$;

- Constante gravitacional: $g=9.81 \mathrm{~ms}^{-2}$;

- Coeficiente de viscosidade cinemática: $\nu=1.0 \times 10^{-6} \mathrm{~m}^{2} \mathrm{~s}^{-1}$;

- Parâmetros de escala: $L=0.1 \mathrm{~m}$ e $U=\sqrt{g L}=0.99045444 \mathrm{~ms}^{-1}$;

- Número de Reynolds: $R e=U L / \nu=99.045,44$;

- $\quad$ Número de Froude: $\operatorname{Fr}=U / \sqrt{g L}=1.0$.

A Figura 5.47 mostra a comparação dos dados experimentais de Koshizuka e Oka [70] e Martin e Moyce [93] com os resultados numéricos 2D nas três malhas para a posição frontal do movimento do fluido $\left(X_{\max }\right)$ versus o tempo.

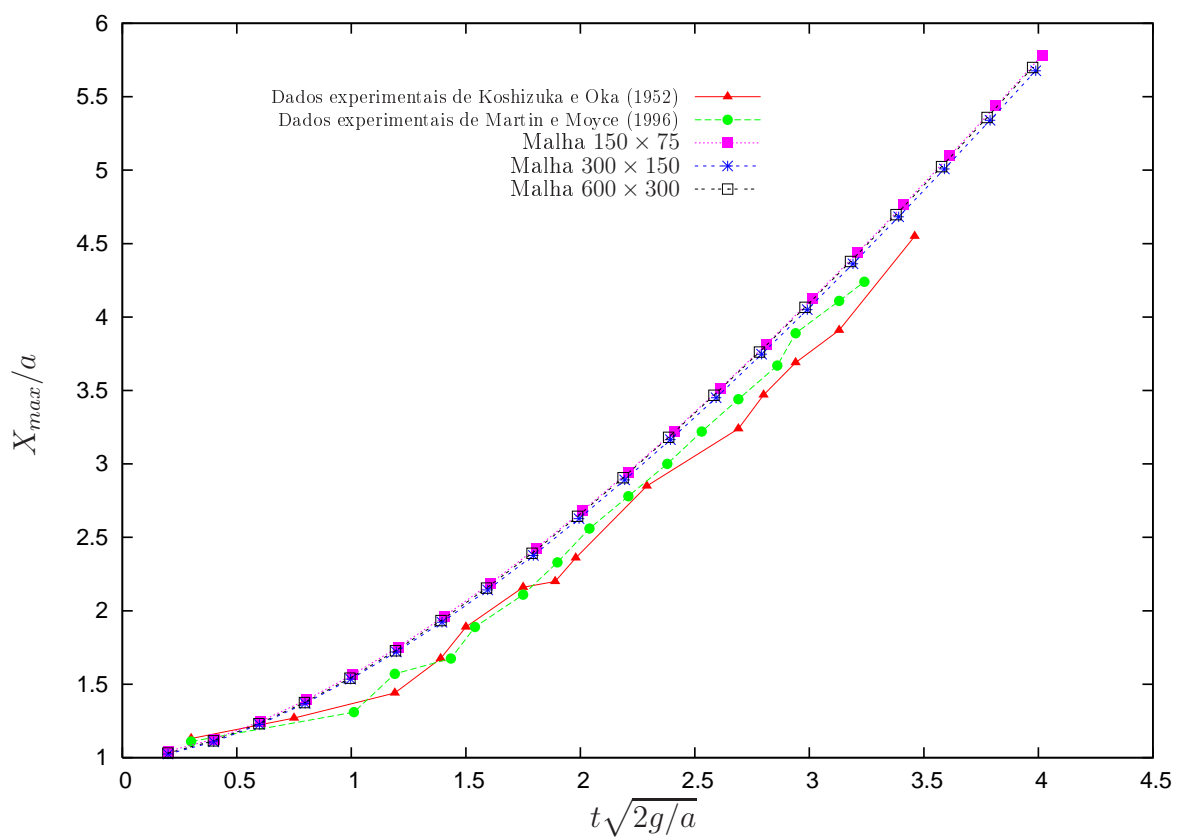

Figura 5.47: Comparação entre as soluções numéricas 2D nas três malhas e os dados experimentais para a posição frontal do movimento do fluido $\left(X_{\max }\right)$.

A Figura 5.48 mostra a comparação dos dados experimentais com os resultados numéricos 2D e 3D (a solução numérica no caso 3D foi computada a partir do corte do plano $x z$ na posição $y=0.05 \mathrm{~m})$.

Observa-se nessas figuras que ambos os resultados numéricos 2D e 3D, computados sem modelagem de turbulência, estão em boa concordância com os dados experimentais, especialmente quando comparados com os resultados de Martin e Moyce. E por esses dados vê-se a confiabilidade das soluções numéricas obtidas com os códigos Freeflow 2D e 3D ambos equipados com o esquema ADBQUICKEST. 


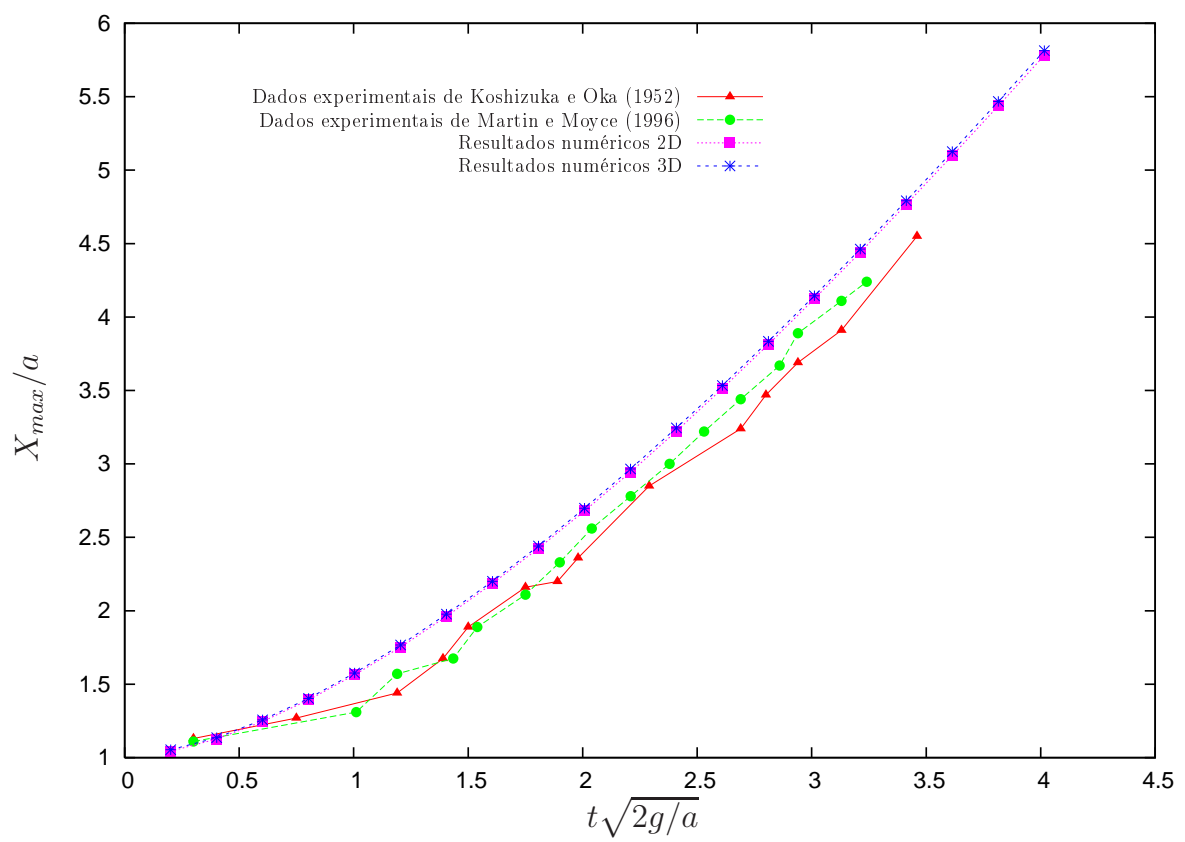

Figura 5.48: Comparação entre os resultados numéricos 2D e 3D com os dados experimentais para a posição frontal do movimento do fluido $\left(X_{\max }\right)$.
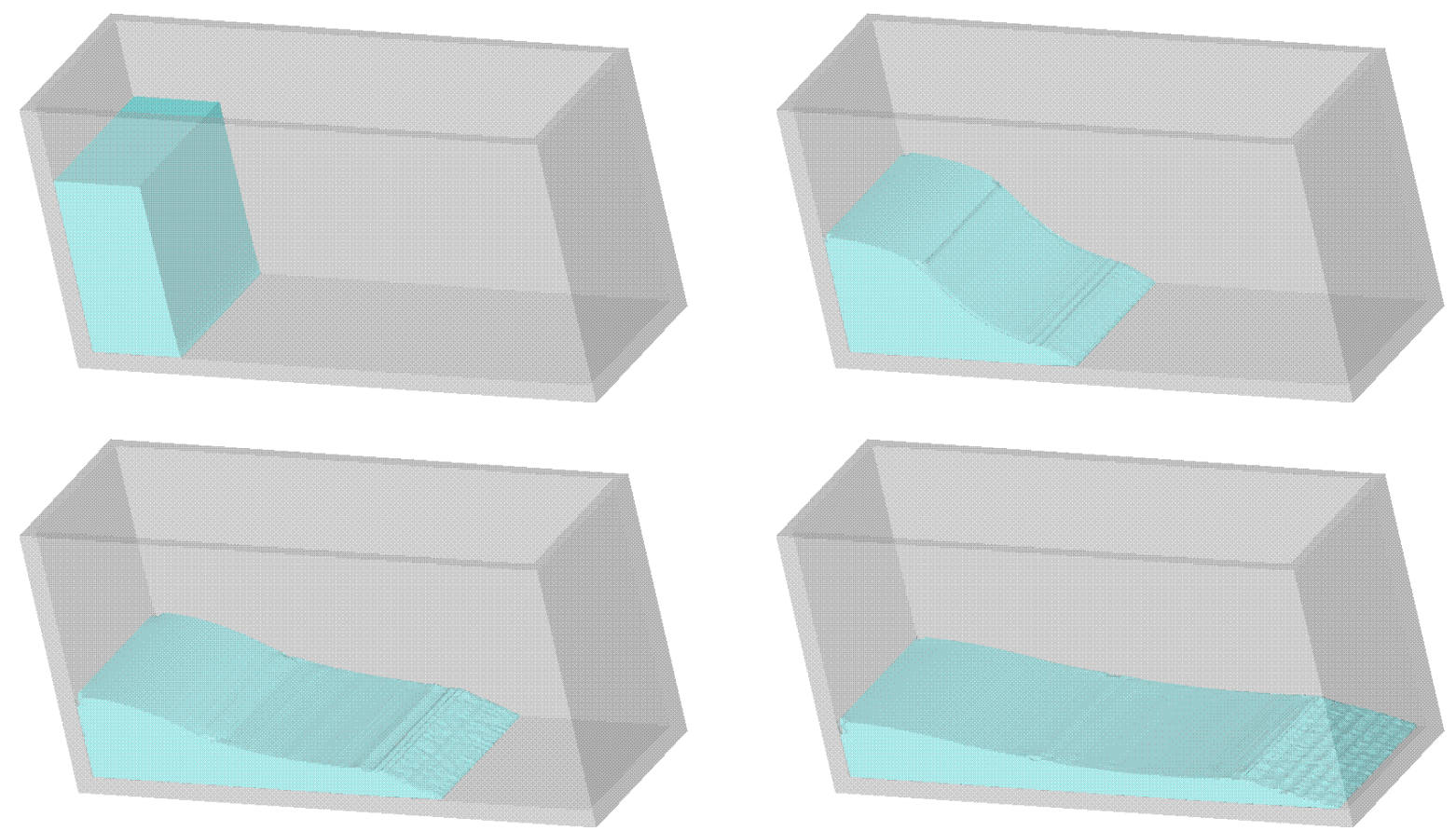

Figura 5.49: Simulação numérica 3D utilizando o esquema ADBQUICKEST ilustrando a evolução da superfície livre em diferentes tempos. 
A Figura 5.49 mostra a simulação numérica em 3D da evolução da superfície livre do fluido nos tempos $t=0.0 \mathrm{~s}, t=1.0 \mathrm{~s}, t=1.5 \mathrm{~s}$ e $t=2.0 \mathrm{~s}$, e as Figuras 5.50 e 5.51 mostram, respectivamente, os contornos de pressão e velocidade $2 \mathrm{D}$ e $3 \mathrm{D}$ nas malhas $150 \times 75$ células e $150 \times 50 \times 75$ células e nos tempos $t=1.0 \mathrm{~s}$ e $t=2.0 \mathrm{~s}$.

\section{Campo de pressão}
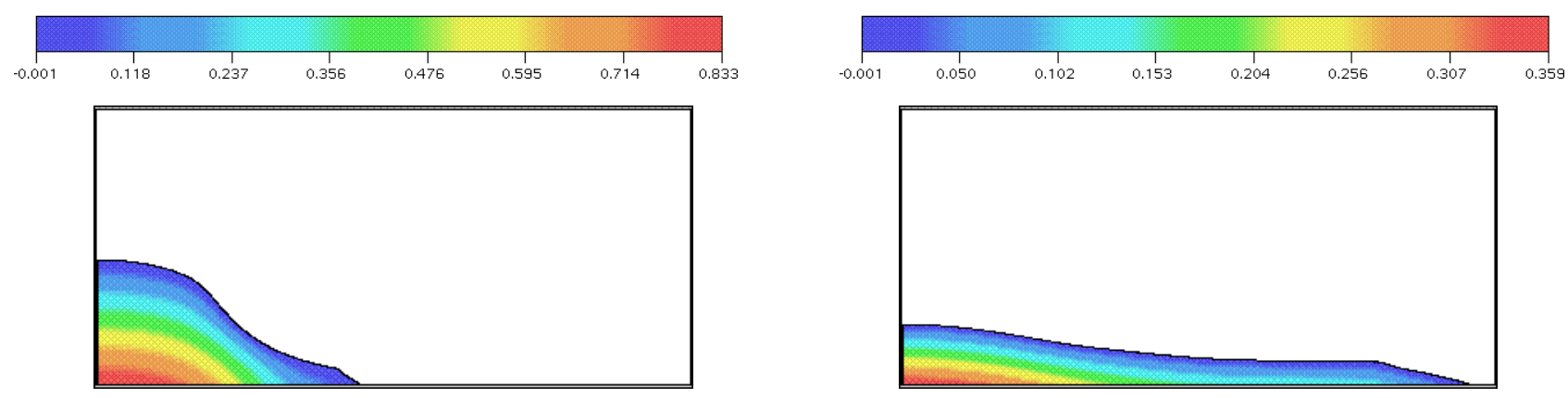

$t=1.0 \mathrm{~s}$

$$
t=2.0 \mathrm{~s}
$$

Campo de velocidade na direção $x$

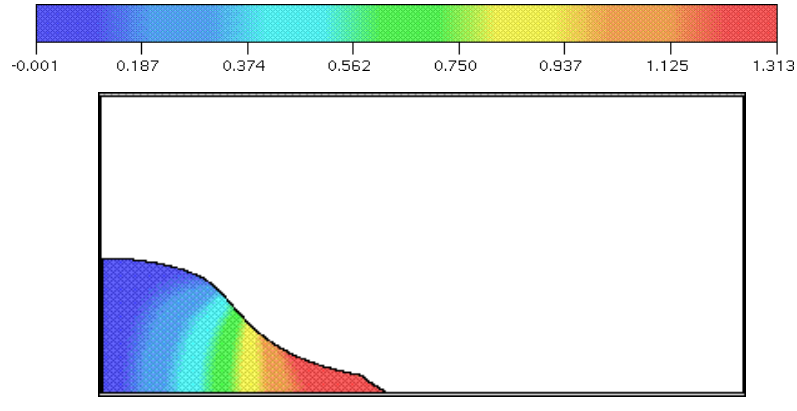

$t=1.0 \mathrm{~s}$

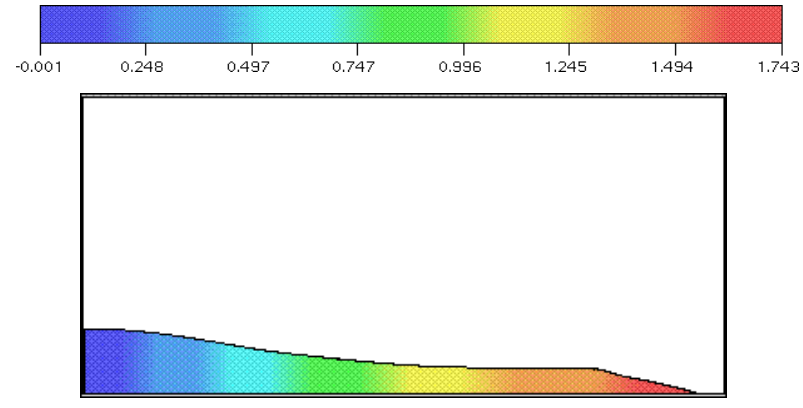

$t=2.0 \mathrm{~s}$

Campo de velocidade na direção $y$

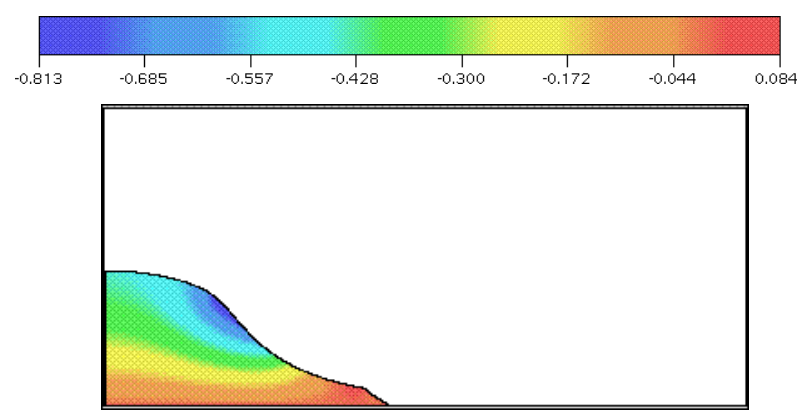

$t=1.0 \mathrm{~s}$

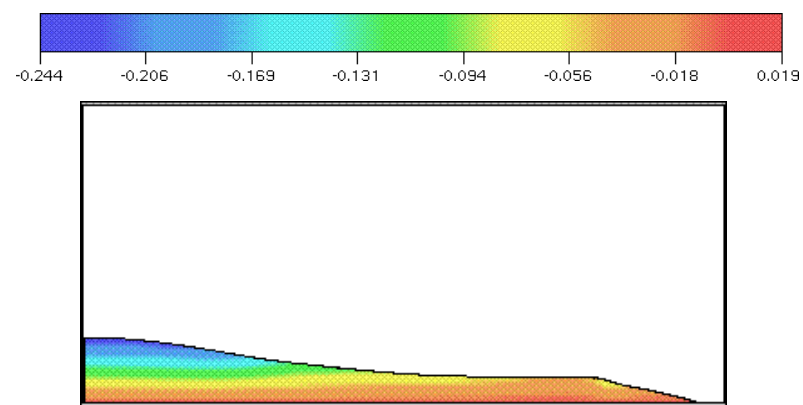

$t=2.0 \mathrm{~s}$

Figura 5.50: Simulação numérica 2D do colapso da coluna de fluido utilizando o esquema ADBQUICKEST com $R e=99.045,44$. 
Campo de pressão
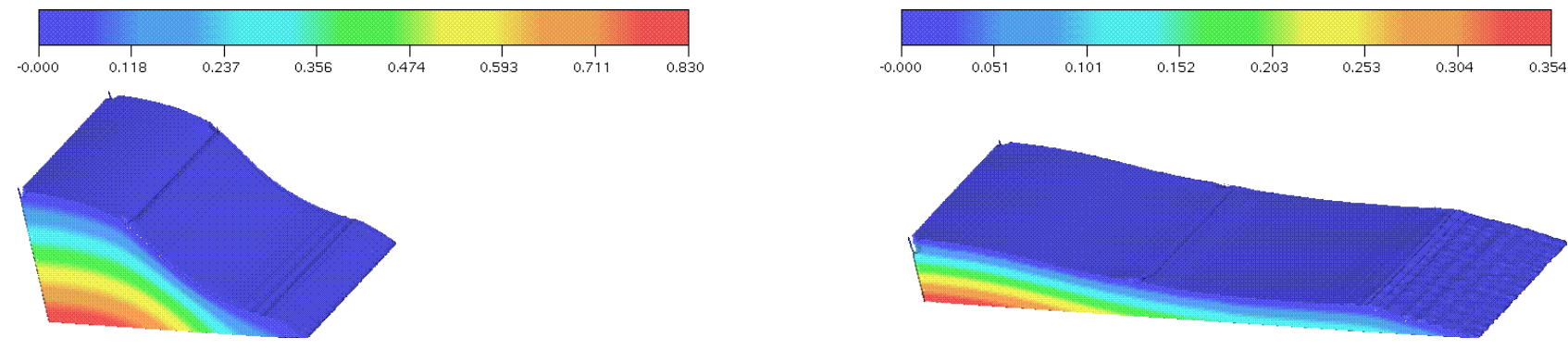

$$
t=1.0 \mathrm{~s}
$$

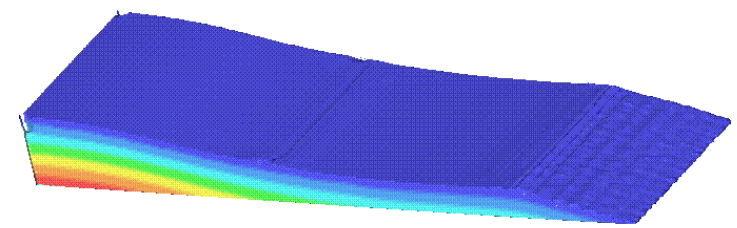

$$
t=2.0 \mathrm{~s}
$$

Campo de velocidade na direção $x$
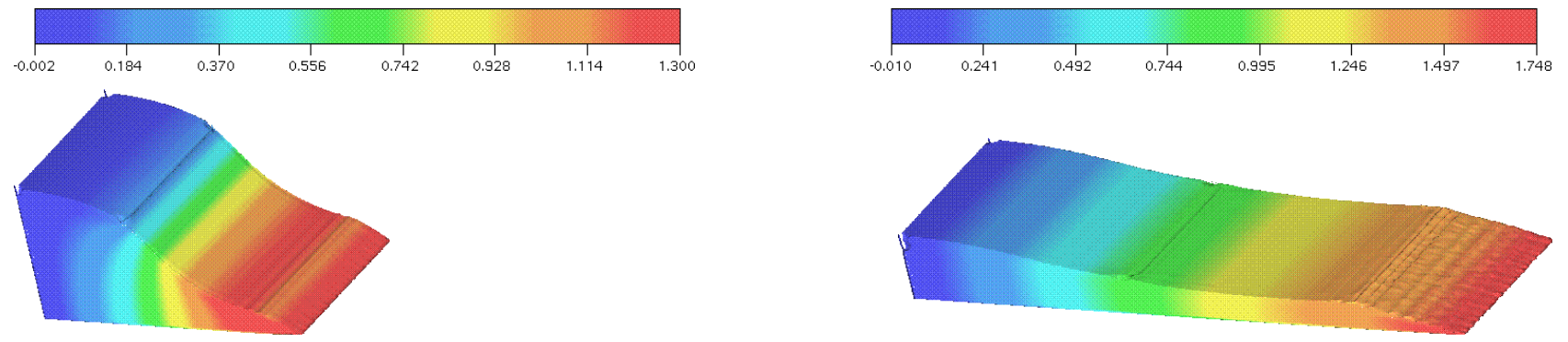

$$
t=1.0 \mathrm{~s}
$$

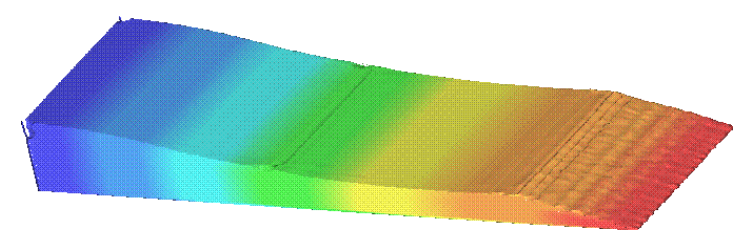

$$
t=2.0 \mathrm{~s}
$$

Campo de velocidade na direção $z$
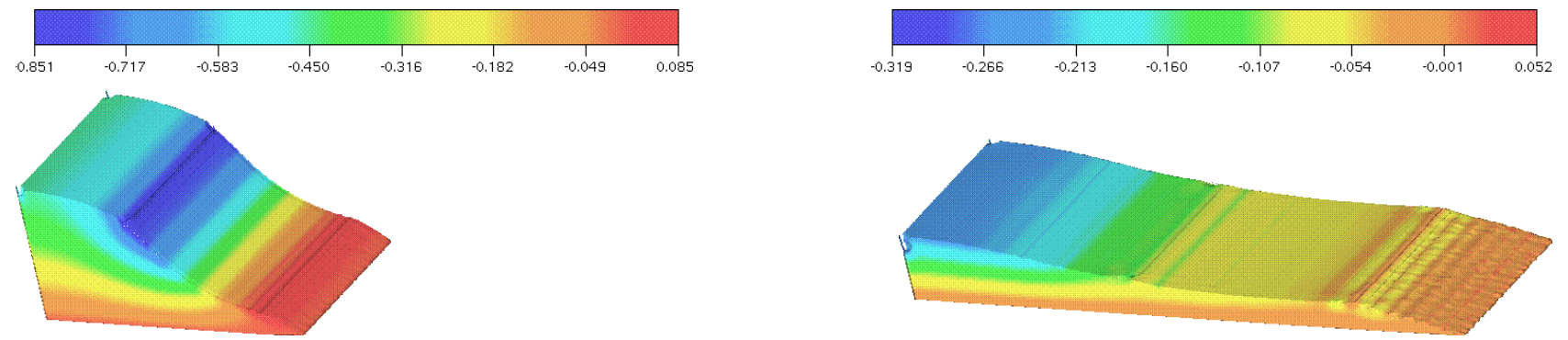

$$
t=1.0 \mathrm{~s}
$$

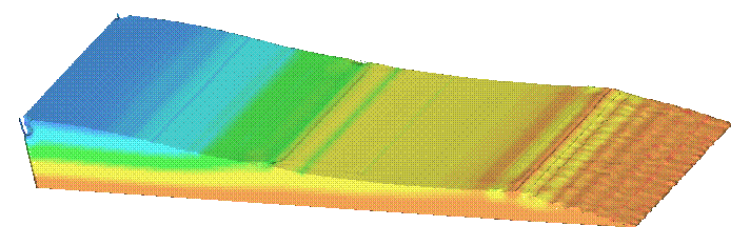

$$
t=2.0 \mathrm{~s}
$$

Figura 5.51: Simulação numérica 3D do colapso da coluna de fluido utilizando o esquema ADBQUICKEST com $R e=99.045,44$. 


\subsubsection{Aplicações: escoamentos 3D com superfícies livres móveis}

Nesta subseção, são apresentadas algumas simulações numéricas de escoamentos 3D com superfícies livres móveis. O objetivo aqui é mostrar que o ambiente de simulação Freeflow 3D atualizado com o esquema "upwind" de alta resolução ADBQUICKEST está apto para simular problemas 3D altamente complexos a altos e baixos número de Reynolds.

\subsubsection{Ressalto hidráulico circular}

Um fenômeno bastante interessante pode ser visto no dia-a-dia ao se abrir a torneira de cozinha que libera um jato circular de água. Esse jato incide perpendicularmente à cuba da pia (superfície rígida) e espalha radialmente a partir do ponto de estagnação e, após um determinado raio, surge uma onda estacionária (descontinuidade súbita), como ilustrado na Figura 5.52. De acordo com Kurihara [72] e Tani [132], essa descontinuidade ocorre porque, durante o espalhamento, a espessura do fluido decresce e, então, a uma certa distância cresce repentinamente sob à ação de um gradiente de pressão adverso. Esse gradiente de pressão causa separação da película em alguma distância radial, levando à formação de um fenônmeno conhecido como ressalto hidráulico circular.

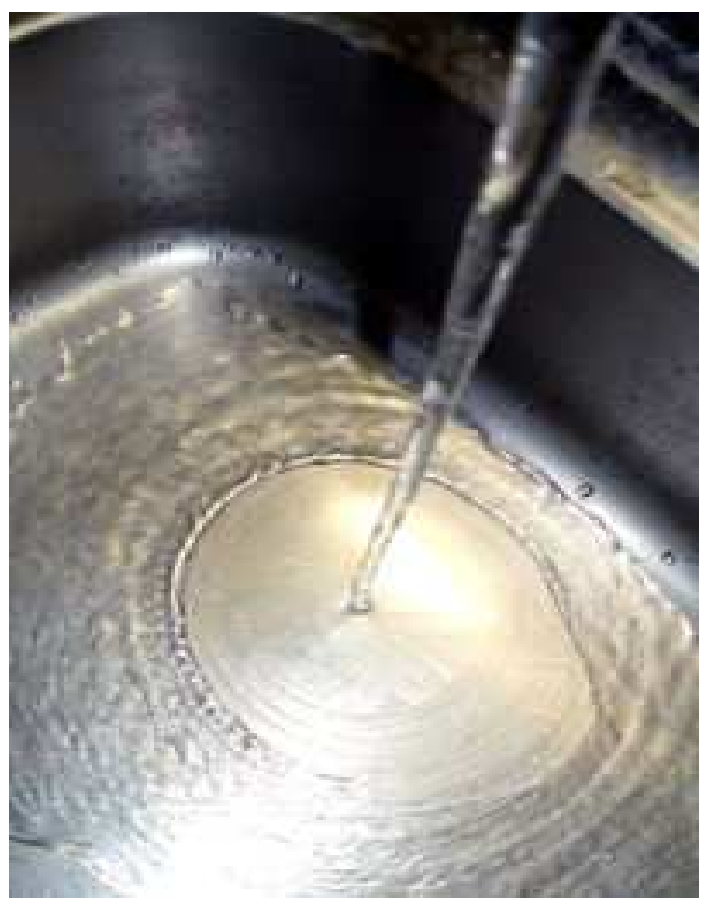

Figura 5.52: Visualização de um exemplo muito comum de ressalto hidráulico circular.

O entendimento dos fenômenos envolvidos nesse problema com superfície livre móvel é de grande interesse comercial, em particular, na indústria. Por exemplo, o sistema de refrigeração automotivo, na qual o escoamento lento do líquido depois do ressalto pode degradar a eficiência do sistema. Nesse tipo de problema, sempre estão presentes o transporte de energia, a transferência de massa e, sobre tudo, a presença de uma descontinuidade no movimento, análoga 
às ondas de choque que aparecem nos escoamentos compressíveis em altas velocidades. Tais peculiaridades mais a presença da superfície livre móvel criam sérias dificuldades no tratamento numérico desse tipo de escoamento. Dessa forma, o conhecimento do campo de velocidades em várias regiões do escoamento é, portanto, indispensável para o bom entendimento dos fenômenos envolvidos.

A primeira tentativa em simular este problema se deve a Rayleigh [110] que derivou as propriedades do ressalto. Provavelmente, o primeiro autor a estudar a influência da viscosidade do fluido sobre o ressalto foi Watson [155]. Desde então, poucos estudos têm sido direcionados para compreender o fenômeno com maior rigor. Ellegaard et al [36] descobriram que, se o jato de fluido é constituído de um líquido mais viscoso que a água, podem-se formar estruturas poligonais estacionárias, quebrando a simetria axial. Avedisian e Zhao [9] realizaram uma simulação experimental do ressalto hidráulico circular a baixa gravidade, e seus resultados mostraram que o diâmetro do ressalto a essa gravidade é maior que aquele em gravidade normal. Bush e Aristoff [16] investigaram a influência da tensão superficial no ressalto. E mais recentemente, Bush et al. [17] fizeram novas experimentações sobre as estruturas do ressalto, ou seja, além das formas poligonais, eles descobriram uma nova classe de ressaltos, como por exemplo, estruturas semelhantes a olhos de gato, trevos de três e quatro folhas, borboletas, entre outras.

Hoje em dia, as formas mais comuns do ressalto hidráulico circular (com película suave após o ressalto (ver Figura 5.53 (a)) e a com instabilidades na película após o ressalto (ver Figura 5.53 (b))) e as várias estruturas poligonais constituem excelentes problemas com superfícies livres para a validação de códigos baseados na técnica "front-tracking". E não é do nosso conhecimento a existência de um código computacional capaz de simular essas várias estruturas. Isso também tem motivado a presente investigação científica.

Para a simulação do ressalto hidráulico circular do tipo mostrado na Figura 5.53 (b), o seguinte modelo foi empregado:

- Dimensão do domínio: $0.6 \mathrm{~m} \times 0.6 \mathrm{~m} \times 0.05 \mathrm{~m}$;

- Diâmetro do injetor: $D=0.08 \mathrm{~m}$;

(O injetor está posicionado a $0.020 \mathrm{~m}$ da base);

- Altura do injetor: $H=0.015 \mathrm{~m}$;

- Velocidade de injeção: $U=1.0 \mathrm{~ms}^{-1}$;

- Constante gravitacional: $g=9.81 \mathrm{~ms}^{-2}$;

- Coeficiente de viscosidade cinemática: $\nu=5.0 \times 10^{-5} \mathrm{~m}^{2} \mathrm{~s}^{-1}$;

- Parâmetros de escala: $D=0.08 \mathrm{~m}$ e $U=1.0 \mathrm{~ms}^{-1}$;

- Número de Reynolds: $R e=D U / \nu=1000$;

- Número de Froude: $F r=U / \sqrt{g L}=0.700357$. 
O código Freeflow 3D, equipado com o esquema ADBQUICKEST, foi executado com uma malha de $120 \times 120 \times 10(\delta x=\delta y=\delta z=0.005 \mathrm{~m})$ células computacionais e tempo final de simulação $t=2.7 \mathrm{~s}$. A simulação numérica resultante é apresentado na Figura 5.54. Pode ser visto a partir dessa figura, que o ressalto hidráulico circular com instabilidades na película após o ressalto pode ser simulado usando o esquema numérico proposto aqui. Isso mostra mais uma vez a utilidade do esquema "upwind" ADBQUICKEST de alta resolução em simular escoamentos 3D altamente complexos com superfícies livres.

(a)

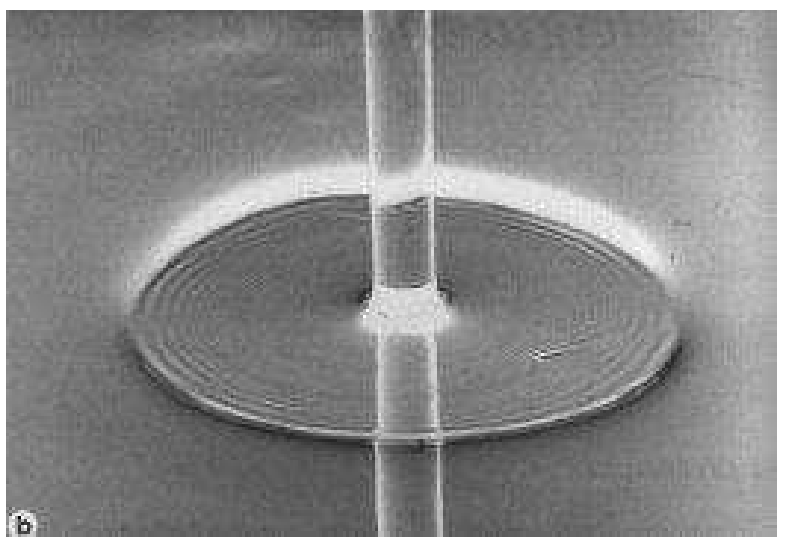

(b)

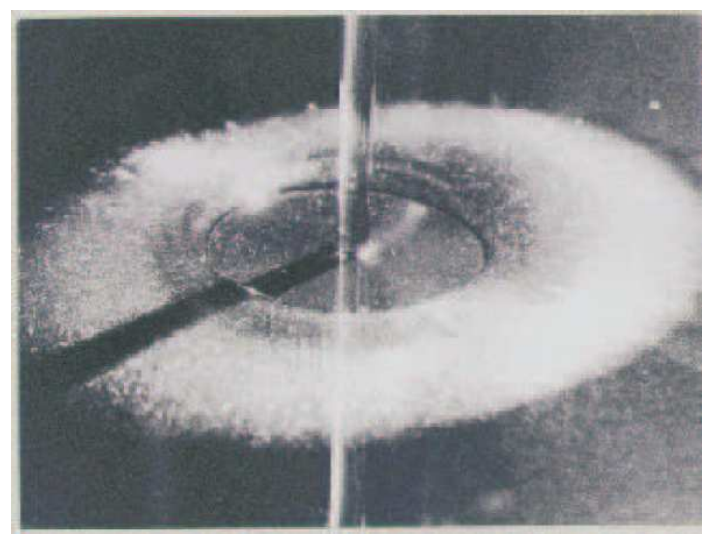

Figura 5.53: Visualização da estrutura do ressalto hidráulico circular a número de Reynolds:(a) moderado; (b) elevado.

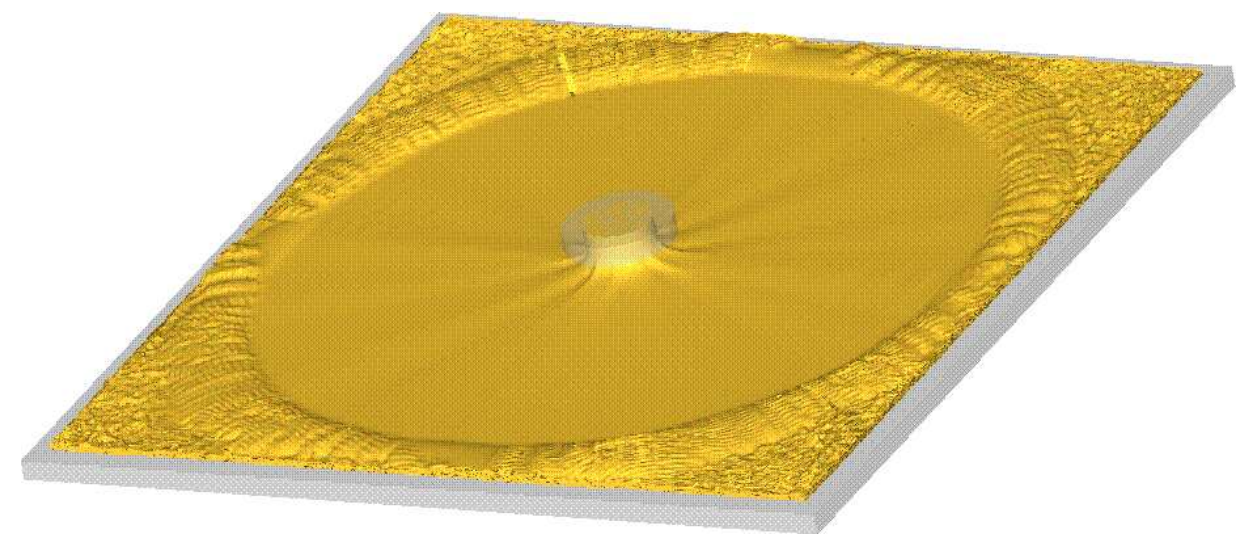

Figura 5.54: Simulação numérica 3D obtida pelo código Freeflow 3D utilizando-se o esquema ADBQUICKEST, ilustrando o ressalto hidráulico circular em $t=2.7 \mathrm{~s}$.

\subsubsection{Experimentos de Taylor}

Esse problema, um antigo experimento realizado por Taylor [135], consiste de um jato incidindo perpendicularmente sobre uma caixa contendo um corpo de fluido (de mesma viscosidade do incidente) em repouso. As simulações foram feitas com o código Freeflow $2 D$ para problemas 
com simetria radial, no qual foi incluído o esquema ADBQUICKEST numa malha $50 \times 100$. A Figura 5.55 mostra a comparação, em dois tempos, entre os experimentos de Taylor e as simulações numéricas deste problema a $R e=200$. Em particular, no caso (c) desta figura, o jato alcança o fundo da caixa formando um estrutura toroidal. É importante observar neste caso que Tomé et al. ([141], Figura 9, p.458) também simulou este problema com superfície livre usando o esquema diferença centrais para os termos advectivos; e seus resultados, entretanto, falharam em capturar essa estrutura complexa.

(a)

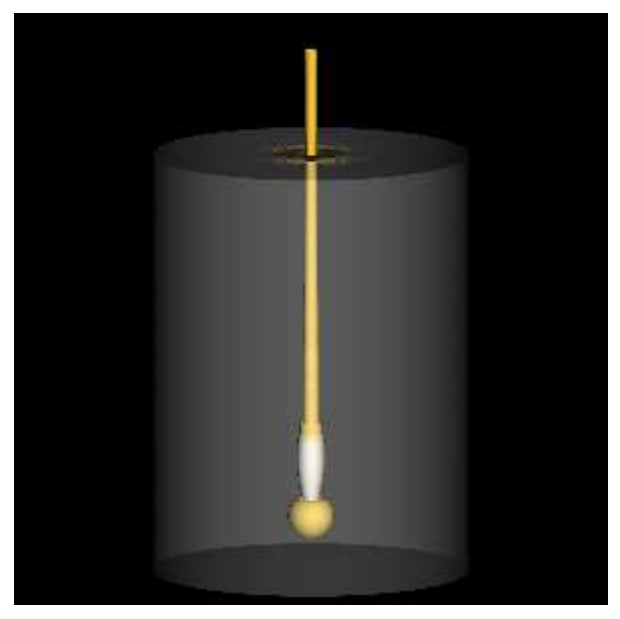

(c)

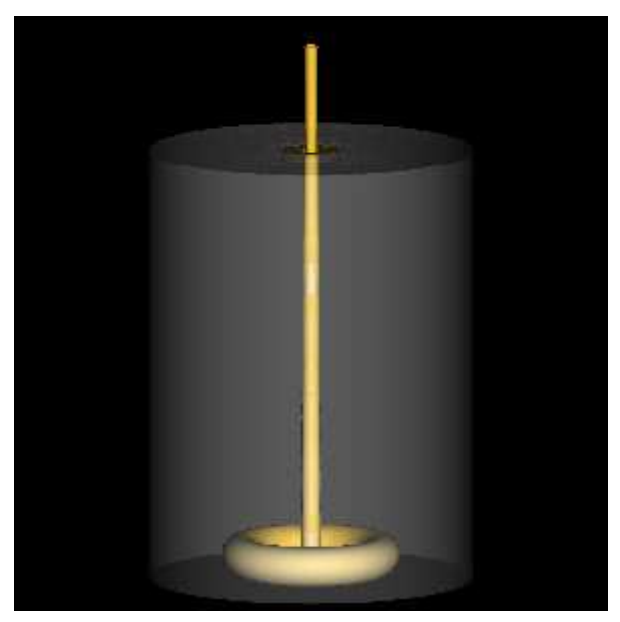

(b)

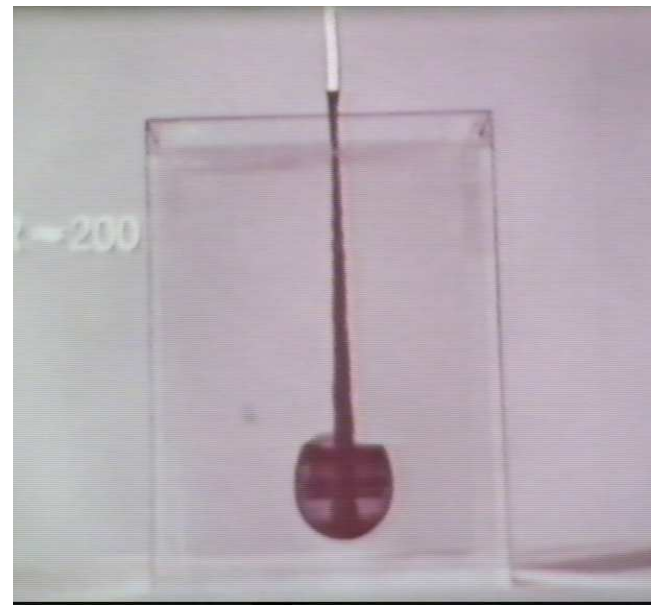

(d)

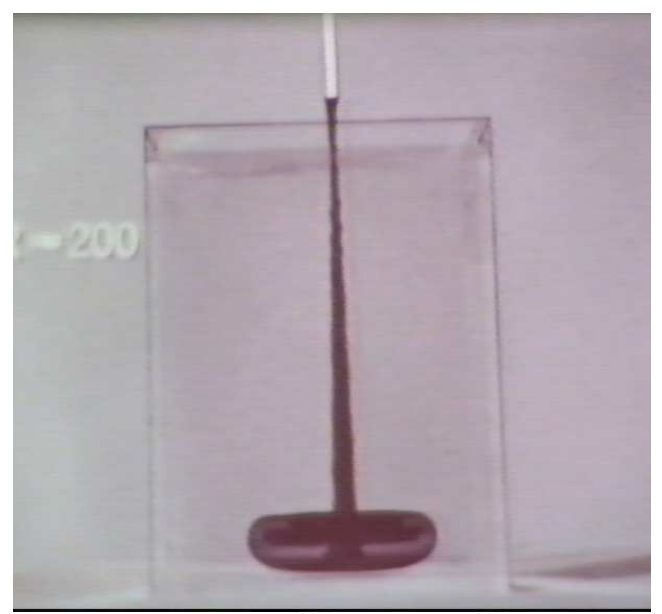

Figura 5.55: Comparação entre a solução numérica obtida pelo esquema ADBQUICKEST, e o experimento realizado por Taylor com $R e=200$ mostrando: (a) a simulação numérica 3D no tempo $t=40 \mathrm{~s}$; (b) a visualização do experimento de Taylor no tempo $t=40 \mathrm{~s}$; (c) simulação numérica 3D no tempo $t=100 \mathrm{~s}$; e (d) a visualização do experimento de Taylor no tempo $t=100 \mathrm{~s}$. 


\subsubsection{Jato oscilante}

As instabilidades físicas (ou flambagem) que aparecem em um jato suficientemente viscoso incidindo numa superfície rígida é um fenômeno interessante em dinâmica dos fluidos com várias aplicações em indústrias de alimentos, processamento de polímero, entre muitas outras áreas. Este problema tem atraído a atenção de muitos pesquisadores desde os tempos de Taylor [134], e resultados numéricos/experimentais mostrando detalhes deste fenômeno para fluidos newtoniano podem ser encontrados nos trabalhos de Cruickshank e Munson [24], Tomé et al. [144], Ribe [112], Nóbrega et al. [101], Huppert [63] e Maleki et al. [90]. Em particular, Cruickshank e Munson [24] realizaram uma série de experimentos para esse tipo de problema e concluíram que o efeito de flambagem ocorre se as seguintes condições estão satisfeitas

$$
R e<1.2 \text { e } H / D>2 \pi
$$

em que $H$ é altura do injetor até o fundo da superfície rígida e $D$ é o diâmetro do injetor. Para a simulação dessas instabilidades físicas os seguintes dados foram empregados:

- Dimensão do domínio: $0.06 \mathrm{~m} \times 0.06 \times 0.11 \mathrm{~m}$;

- Diâmetro do injetor: $D=0.006 \mathrm{~m}$

(O injetor está posicionado a $H=0.11 \mathrm{~m}$ da base);

- Velocidade de injeção: $U=1.0 \mathrm{~ms}^{-1}$;

- Constante gravitacional: $g=9.81 \mathrm{~ms}^{-2}$;

- Coeficiente de viscosidade cinemática: $\nu=1.2 \times 10^{-2} \mathrm{~m}^{2} \mathrm{~s}^{-1}$;

- Parâmetros de escala: $D=0.006 \mathrm{~m} \mathrm{e} U=1.0 \mathrm{~ms}^{-1}$;

- Número de Reynolds: $R e=D U / \nu=0.5$.

Na simulaçao desse problema são utilizados uma malha de $100 \times 100 \times 100(\delta x=\delta y=\delta z=$ $0.001 \mathrm{~m}$ ) células computacionais e tempo de simulação $t=1.5 \mathrm{~s}$. Como pode ser observado na Figura 5.56, as instabilidades físicas foram capturadas com sucesso a esse número de Reynolds. Isto mostra que o esquema ADBQUICKEST, embora proposto para resolver problemas a altos valores do número de Reynolds, pode ser utilizado também, com sucesso, na simulação de escoamentos a baixos número de Reynolds. 

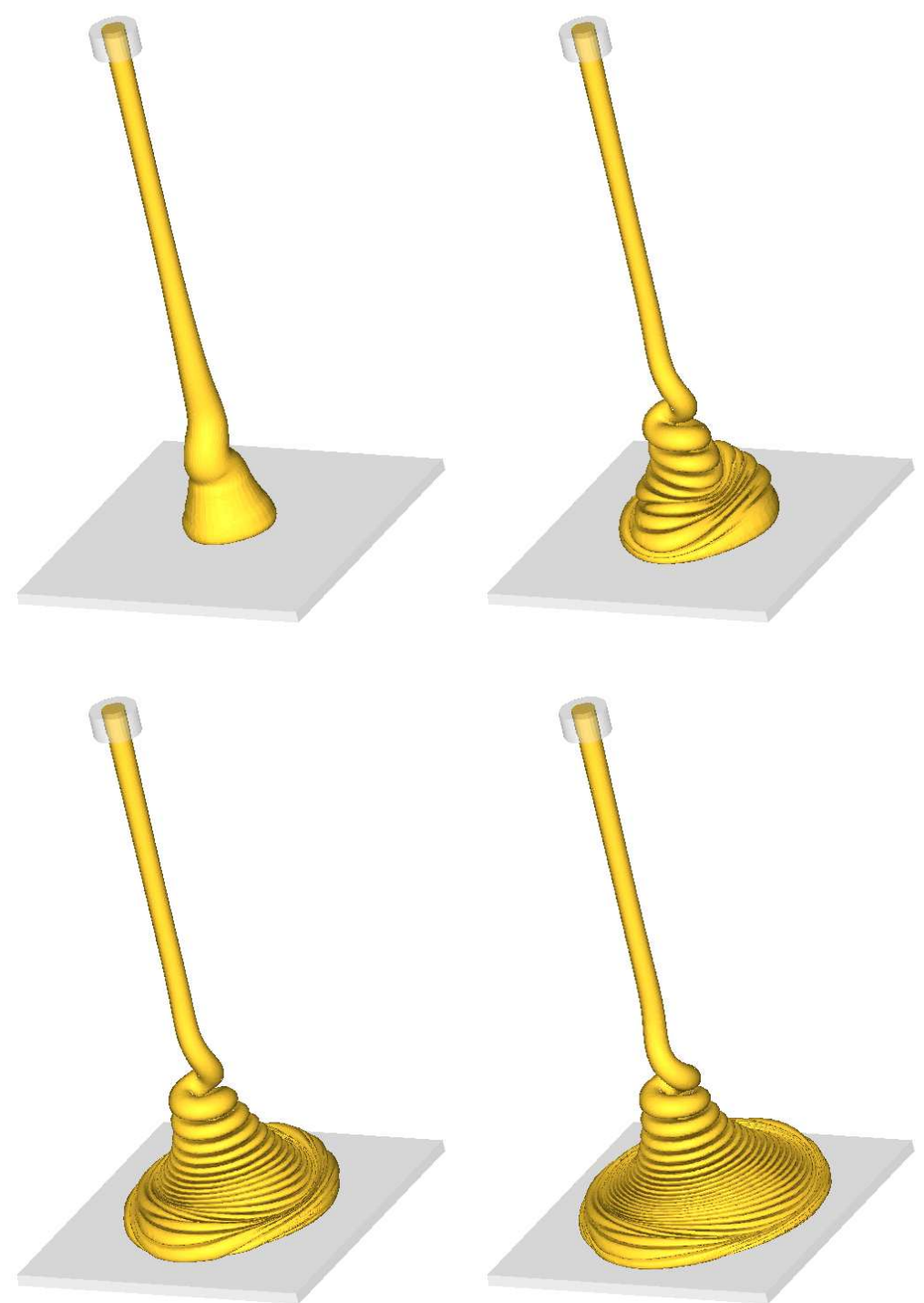

Figura 5.56: Simulação numérica 3D do jato circular oscilante em diferentes tempos. 


\subsubsection{Colapso de um bloco de fluido com obstáculo}

O objetivo aqui é testar o código Freeflow 3D atualizado com o esquema ADBQUICKEST e sem modelagem de turbulência, para problemas complexos a alto número de Reynolds. Esse problema é um exemplo clássico da interação fluido-estrutura.

Interação fluido-estrutura é um tópico importante em muitos campos da engenharia. Exemplos incluem o estudo de ondas contra estruturas (ver Dalrymple et al. [25] e Fontaine et al. [46]), e a simulação de quebra de ondas por Löhner et al. [88]. Uma situação bastante simplificada de fluido-estrutura e útil para validar métodos numéricos é o efeito combinado da superfície livre do fluido e sua viscosidade, tal como o choque de uma onda de superfície contra um obstáculo (ver Gómez-Gesteira e Dalrymple[51] e Lin et al. [86]).

Um tanque de $1.6 \mathrm{~m}$ de comprimento, $0.6 \mathrm{~m}$ de largura e $0.6 \mathrm{~m}$ de altura contém dois blocos de fluido (água) em equilíbrio hidrostático. O primeiro com $0.3 \mathrm{~m}$ de comprimento por $0.6 \mathrm{~m}$ de largura e $0.3 \mathrm{~m}$ de altura e o segundo $1.3 \mathrm{~m}$ de comprimento por $0.6 \mathrm{~m}$ de largura e $0.03 \mathrm{~m}$ de altura. Dentro deste tanque há uma estrutura fixa (pilastra) de $0.12 \mathrm{~m}$ de comprimento $\times$ $0.12 \mathrm{~m}$ de largura $\times 0.6 \mathrm{~m}$ de altura posicionada no fundo a $0.5 \mathrm{~m}$ do primeiro bloco de fluido e a $0.24 \mathrm{~m}$ das paredes laterais (ver Figura 5.57). No tempo $t=0 \mathrm{~s}$, os dois blocos de fluido são instantaneamente colocados à ação da gravidade sofrendo colapso. O movimento resultante do volume da água após o colapso em diferentes tempos está ilustrado na Figura 5.58. Os parâmetros adimensionais utilizado neste problema foram $\operatorname{Re}=514.655,22$ e $F r=1.0$. Os dados restantes estão dispostos na modelo abaixo.

- Dimensão do domínio: $1.6 \mathrm{~m} \times 0.6 \times 0.6 \mathrm{~m}$;

- Constante gravitacional: $g=9.81 \mathrm{~ms}^{-2}$;

- Coeficiente de viscosidade cinemática: $\nu=1.0 \times 10^{-6} \mathrm{~m}^{2} \mathrm{~s}^{-1}$;

- Parâmetros de escala: $L=0.30 \mathrm{~m}$ e $U=\sqrt{g L}=1.715517 \mathrm{~ms}^{-1}$;

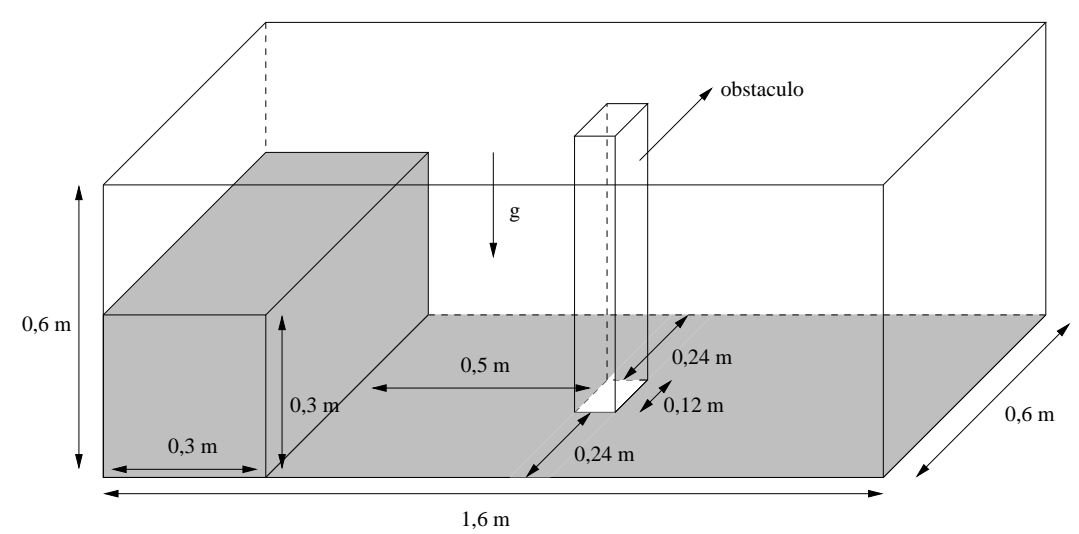

Figura 5.57: Diagrama esquemático do problema do colapso de uma bloco de fluido com obstáculo. 
O código FreeFlow-3D incrementado com o esquema ADBQUICKEST e sem modelagem da turbulência simulou este problema numa malha $160 \times 60 \times 60(\delta x=\delta y=\delta z=0.010 \mathrm{~m})$ e com os dados de entrada mostrados acima. A Figura 5.58 exibe, em diferentes tempos, a simulação numérica da interação fluido envolvendo superfície livre com a estrutura.
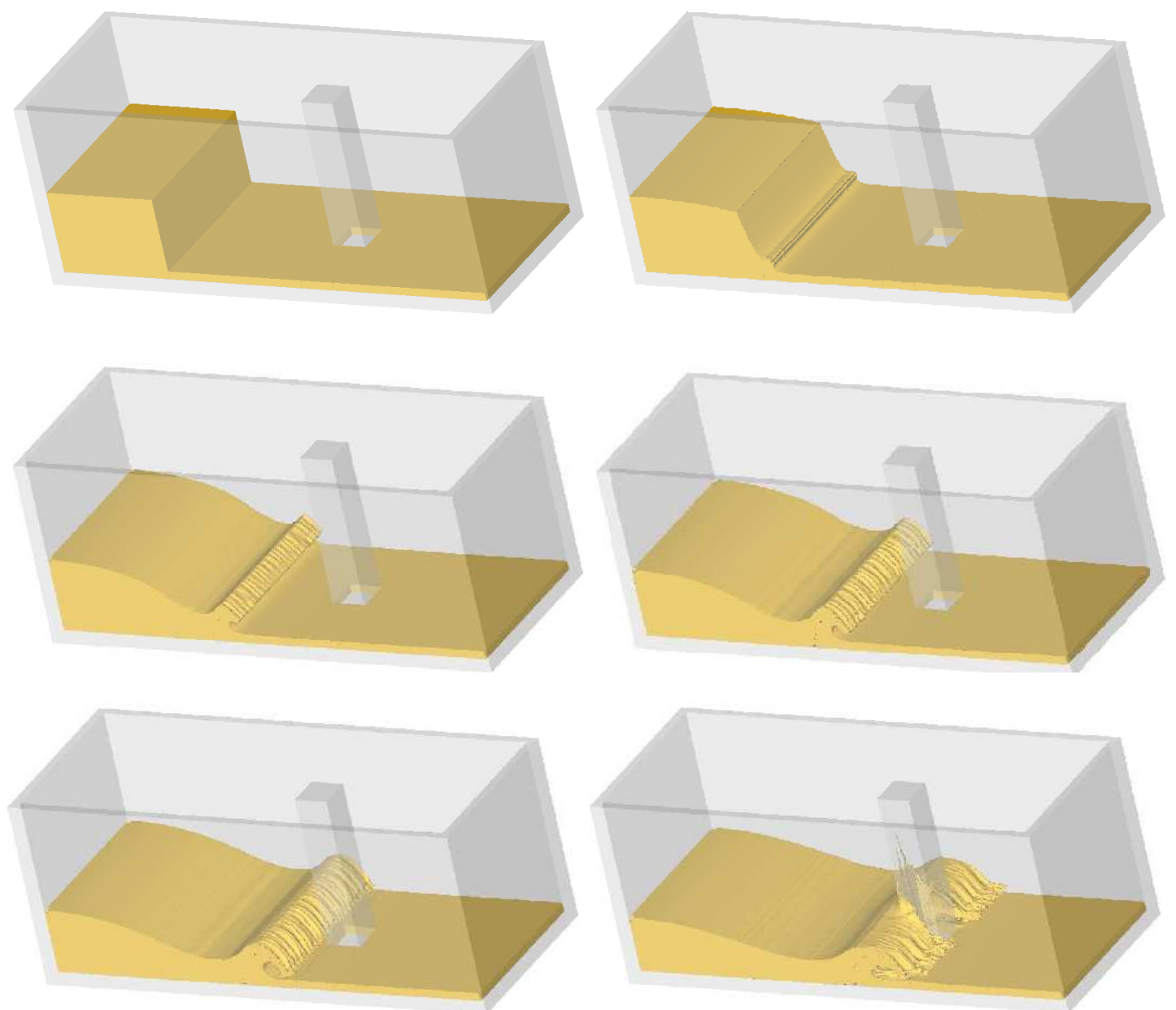

Figura 5.58: Simulação numérica 3D do colapso de uma bloco de fluido com obstáculo em diferentes tempos.

\subsubsection{Comentários}

O esquema ADBQUICKEST foi testado em escoamentos 2D e 3D laminares, sendo que a maioria deles envolveu superfícies livres móveis. Os resultados numéricos mostraram uma boa concordância quando comparados com soluções analíticas e dados experimentais existentes na literatura. Isso, mais uma vez, evidenciou a robustez e flexibilidade do esquema ADBQUICKEST em resolver problemas laminares com superfícies livres a uma variedade de Reynolds. Os resultados numéricos sobre a expansão brusca 2D e o jato livre 2D incidindo sobre uma superfície rígida estão publicados no periódico Communications in Numerical Methods in Engineering (Ferreira et al. [43]) e os demais resultados 3D estão disponíveis no periódico Mathematics and Computers in Simulation (Ferreira et al. [40]). 


\subsection{Simulação de escoamentos turbulentos 2D e 3D}

Nesta seção são apresentados os resultados das simulações de problemas de escoamentos incompressíveis 2D e 3D em regime turbulento envolvendo superfícies livres móveis. Esses resultados foram obtidos com os códigos Freeflow 2D e 3D atualizados com o esquema ADBQUICKEST e o modelo de turbulência $\kappa-\varepsilon$. É importante ressaltar aqui que, diferentemente de como é feito em muitos códigos comerciais, todos os termos não-lineares nas equações de transporte são discretizados pelo esquema ADBQUICKEST apresentado na seção 3.5.

\subsubsection{Expansão brusca 2D}

O problema da expansão brusca 2D apresentado na subseção 5.2.1 é agora retomado e simulado com as modelagens ADBQUICKEST e $\kappa-\varepsilon$ da turbulência. Novamente, o objetivo é estimar o comprimento da recirculação principal $x_{1}$ (ver Figura 5.59). Nesse caso, os resultados numéricos obtidos são comparados com os dados experimentais de Eaton e Johnston [33].

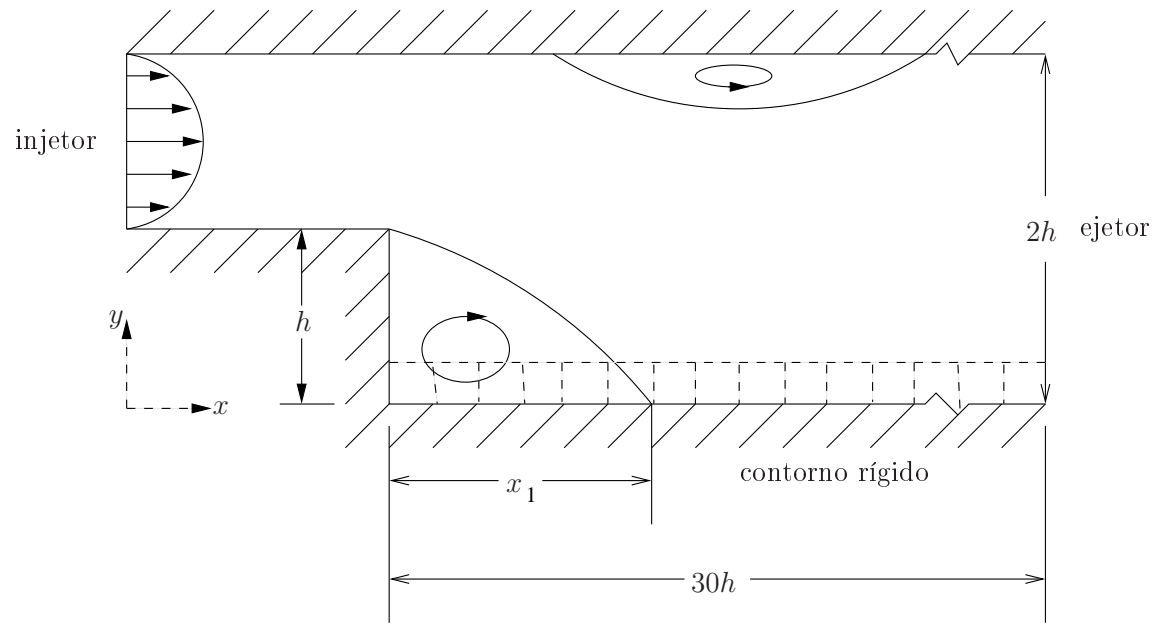

Figura 5.59: Geometria do problema da expansão brusca turbulento.

Analogamente ao caso laminar (ver subseção 5.2.1), o perfil de velocidade imposto na entrada do canal é parabólico (ver equação (5.14)), e o número de Reynolds utilizado nessa simulação é o número de Reynolds numérico, $R e_{\text {num }}=1.98 \times 10^{5}$ que equivale ao Reynolds do problema, $R e=1.32 \times 10^{5}$ (ver equação (5.19)). O seguinte modelo foi adotado:

- Dimensão do domínio: $4.0 \mathrm{~m} \times 0.2 \mathrm{~m}$;

- Entrada do injetor: $h=0.1 \mathrm{~m}$;

- Velocidade de injeção: $U_{\max }=1.0 \mathrm{~ms}^{-1}$;

- Coeficiente de viscosidade cinemática: $\nu \approx 2.272 \times 10^{-6} \mathrm{~m}^{2} \mathrm{~s}^{-1}$;

- Parâmetros de escala: $2 h=0.2 \mathrm{~m}$ e $U_{\max }=1.0 \mathrm{~ms}^{-1}$; 
O problema foi simulado utilizando três malhas diferentes, a saber, a malha grossa $(200 \times 15$ células, $\delta x=\delta y=0.02 \mathrm{~m})$; a malha intermediária $(400 \times 30$ células, $\delta x=\delta y=0.01 \mathrm{~m})$; e a malha fina $(800 \times 60$ células, $\delta x=\delta y=0.005 \mathrm{~m})$. O número de Reynolds crítico $y_{c}^{+}$, solução de (2.88), e as constantes $K$ e $B$, nas leis de parede, assumiram os seguintes valores: $y_{c}^{+} \approx 10.804871, K=0.41$ e $B=5.0$, respectivamente. O critério de convergência para a equação de Poisson e o tamanho do passo temporal utilizados nas três simulações foram $1.0 \times 10^{-12}$ e $4.167 \times 10^{-5}$, respectivamente. Os processos foram finalizados quando o escoamento atingiu o regime estacionário. O tempo final de simulação foi $t=1000 \mathrm{~s}$.

Tabela 5.4: Valores do comprimento da recirculação, $x_{1}$.

\begin{tabular}{|c|c|c|c|c|}
\hline Malha & ADBQUICKEST & CUBISTA & WACEB & VONOS \\
\hline \hline Grossa & 6.86 & 7.13 & 7.10 & 6.12 \\
\hline Intermediária & 6.03 & 6.10 & 6.06 & 5.76 \\
\hline Fina & 5.50 & 5.51 & 5.50 & 5.42 \\
\hline
\end{tabular}

A Tabela 5.4 apresenta os valores do comprimento da recirculação $x_{1}$, nas três malhas, usando quatro esquemas advectivos, incluindo o esquema ADBQUICKEST. Os dados dessa tabela foram comparados com o dado numérico de Thangam e Speziale [138], cujo valor adimensional é $x_{1}=6.0$, e com o dado experimental de Eaton e Johnston [33], cujo valor adimensional é $x_{1}=7.1$. Como pode-se observar a partir dessa tabela, os resultados obtidos com o modelo $\kappa-\varepsilon$ subestimou o valor de $x_{1}$ em aproximadamente $20-25 \%$, e o esquema CUBISTA na malha grossa forneceu um valor acima de 7.1. Este comportamento do modelo está em concordância com os dados de Speziale [128] e de Thangam e Speziale [138]. Observa-se também, dessa mesma tabela, que os esquemas WACEB, CUBISTA e ADBQUICKEST apresentaram melhores resultados, enquanto que o resultado com esquema VONOS não foram bons. Esse dado ruim obtido com o esquema VONOS pode ser atribuído ao fato dele não ser um esquema TVD.

Tabela 5.5: Outros valores do comprimento da recirculação.

\begin{tabular}{|c|c|c|c|c|c|}
\hline Esquema & HOAB & STOIC & SMART & CLAM & FOU \\
\hline \hline$x_{1}$ & 5.405 & 5.396 & 5.377 & 5.357 & 4.607 \\
\hline
\end{tabular}

Para simples comparação, a Tabela 5.5 mostra valores do comprimento $x_{1}$ obtidos com outros esquemas bem conhecidos na literatura: o esquema HOAB ("High Order Accurate and Bounded") (Wei el al. [157]), o STOIC ("Second- and Third-Order Interpolation for Convection") (Darwish [26]), o SMART ("Sharp and Monotonic Algorithm for Realistic Transport") 
(Gaskell e Lau [47]), o CLAM (van Leer [148]) e o esquema FOU ("First Order Upwind") (Courant et al. [23]). Como pode ser confirmado por essa tabela, o esquema ADBQUICKEST, de fato, forneceu resultados consistentes. Além disso, um teste de convergência da solução numérica obtida com o esquema ADBQUICKEST nas três malhas foi realizado e o resultado está mostrado na Figura 5.60. Vê-se nessa figura que ambos o perfil de velocidade e o comprimento $x_{1}$ convergiram a medida que se refinou a malha. As Figuras 5.61 e 5.62 ilustram os campos de velocidade na direção $x$ e $y$, respectivamente, obtidos com o esquema ADBQUICKEST.

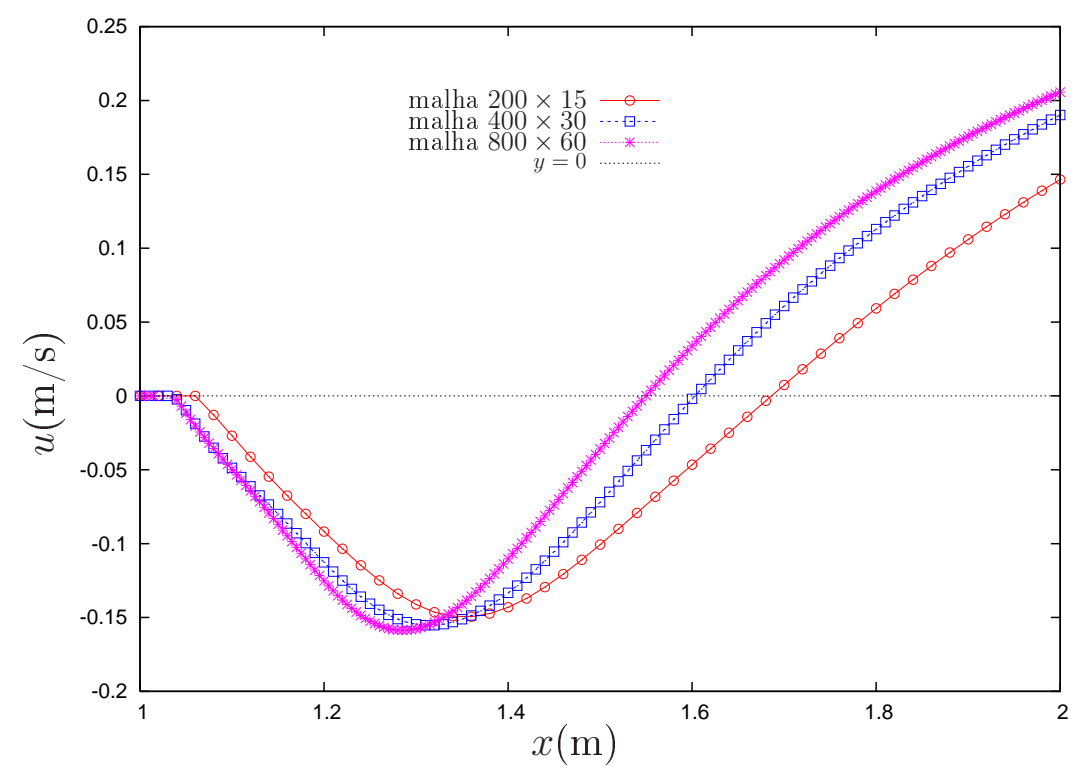

Figura 5.60: Perfis da componente $u$ de velocidade do problema da expansão brusca no regime turbulento, mostrando a convergência da solução numérica nas três malhas usando o esquema ADBQUICKEST.

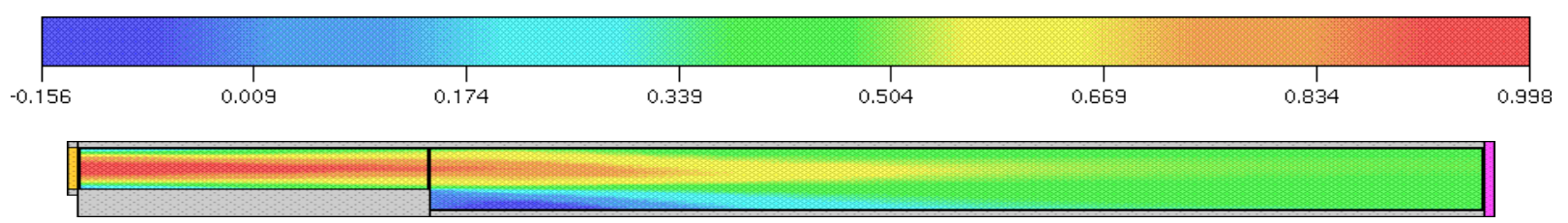

Figura 5.61: Problema da expansão brusca ilustrando o perfil da componente $u$ de velocidade com $R e=1.32 \times 10^{5}$.

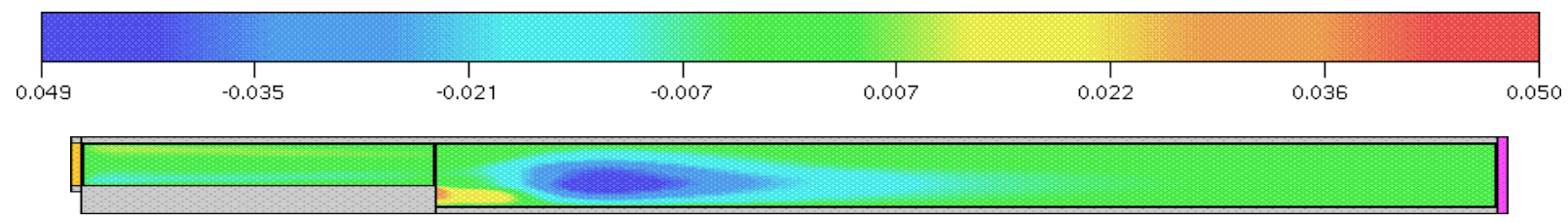

Figura 5.62: Problema da expansão brusca ilustrando o perfil da componente $v$ de velocidade com $R e=1.32 \times 10^{5}$. 


\subsubsection{Jato livre 2D sobre uma superfície rígida impermeável}

O problema de um jato livre 2D incidindo numa superfície rígida impermeável apresentado na subseção 5.2.2 é retomado e simulado usando o código Freeflow $2 D$ equipado com as modelagens ADBQUICKEST e $\kappa-\varepsilon$ da turbulência. Novamente, o objetivo é comparar a altura da superfície livre computada com a solução analítica de Watson [155] para o caso do problema em regime turbulento.

Watson deduziu a seguinte relação explícita para a altura $h$ entre a superfície rígida e a superfície livre do fluido para o caso em que o escoamento é turbulento

$$
h(x)= \begin{cases}\frac{81(7 A)^{\frac{1}{4}} k}{800}\left(\frac{\nu}{Q}\right)^{\frac{1}{4}}(x+l), & \text { se } \quad x \geq x_{0}, \\ a+\left(1-\frac{A}{k}\right) \delta, & \text { se } x<x_{0},\end{cases}
$$

em que

$$
\begin{gathered}
x_{0}=\frac{320(9 A-2)}{81 \times 7^{\frac{1}{4}} A^{\frac{5}{4}}} a R e^{\frac{1}{4}}, \\
l=\frac{160(1-2 A)}{9 \times 7^{\frac{1}{4}} A^{\frac{5}{4}}} a R e^{\frac{1}{4}}
\end{gathered}
$$

e

$$
\delta(x)=\left(\frac{81}{320(9 A-2)}\right)^{\frac{4}{5}} 7^{\frac{1}{5}} k\left(\frac{a \nu}{Q}\right)^{\frac{1}{5}} x^{\frac{4}{5}},
$$

na qual $A=0.239$ e $k=0.260$.

O modelo adotado para a simulação é

- Dimensão do domínio: $0.2 \mathrm{~m} \times 0.05 \mathrm{~m}$;

- Diâmetro do injetor: $L=0.01 \mathrm{~m}$;

- Velocidade de injeção: $U=1.0 \mathrm{~ms}^{-1}$;

- Constante gravitacional: $g=9.81 \mathrm{~ms}^{-2}$;

- Coeficiente de viscosidade cinemática: $\nu=2.0 \times 10^{-7} \mathrm{~m}^{2} \mathrm{~s}^{-1}$;

- Parâmetros de escala: $L=0.01 \mathrm{~m}$ e $U=1.0 \mathrm{~ms}^{-1}$;

- Altura do injetor a partir da superfície rígida: $H=0.015 \mathrm{~m}$;

- Número de Reynolds: $R e=U L / \nu=50000$;

- Número de Froude: $F r=U / \sqrt{g L}=3.19275$.

Nas simulações, foram utilizadas três malhas, a saber: malha grossa $(200 \times 50$ células, 
$\delta x=\delta y=0.001 \mathrm{~m})$; malha intermediária $(400 \times 100$ células, $\delta x=\delta y=0.0005 \mathrm{~m})$; e malha fina $(800 \times 200$ células, $\delta x=\delta y=0.00025 \mathrm{~m})$. O número de Reynolds crítico $y_{c}^{+}$, e as constantes $K$ e $B$, nas leis de parede, assumiram os seguintes valores: $y_{c}^{+} \approx 10.804871, K=0.41$ e $B=5.0$. O critério de convergência para a equação de Poisson foi $1.0 \times 10^{-12}$ e o tamanho do passo temporal $3.125 \times 10^{-6}$ para as três simulações. O tempo final de simulação foi $t=1.0 \mathrm{~s}$.

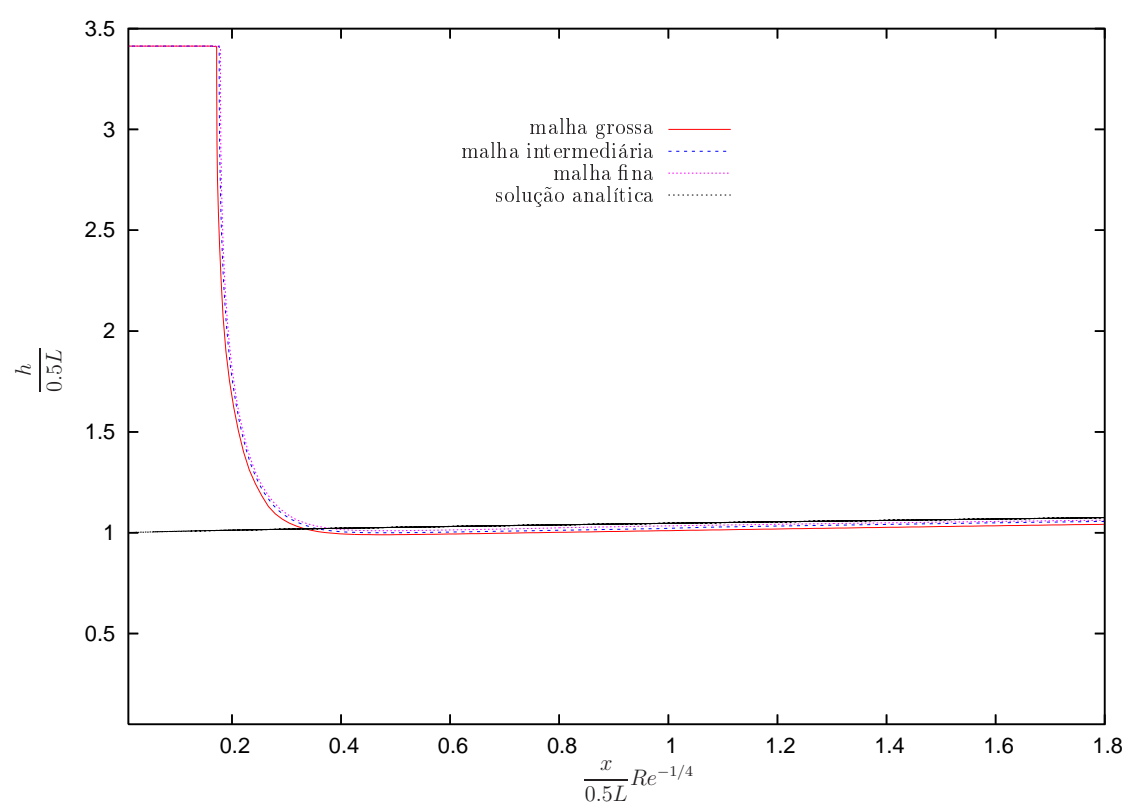

Figura 5.63: Comparação entre as soluções numéricas nas três malhas utilizando o esquema ADBQUICKEST e a solução analítica de Watson.

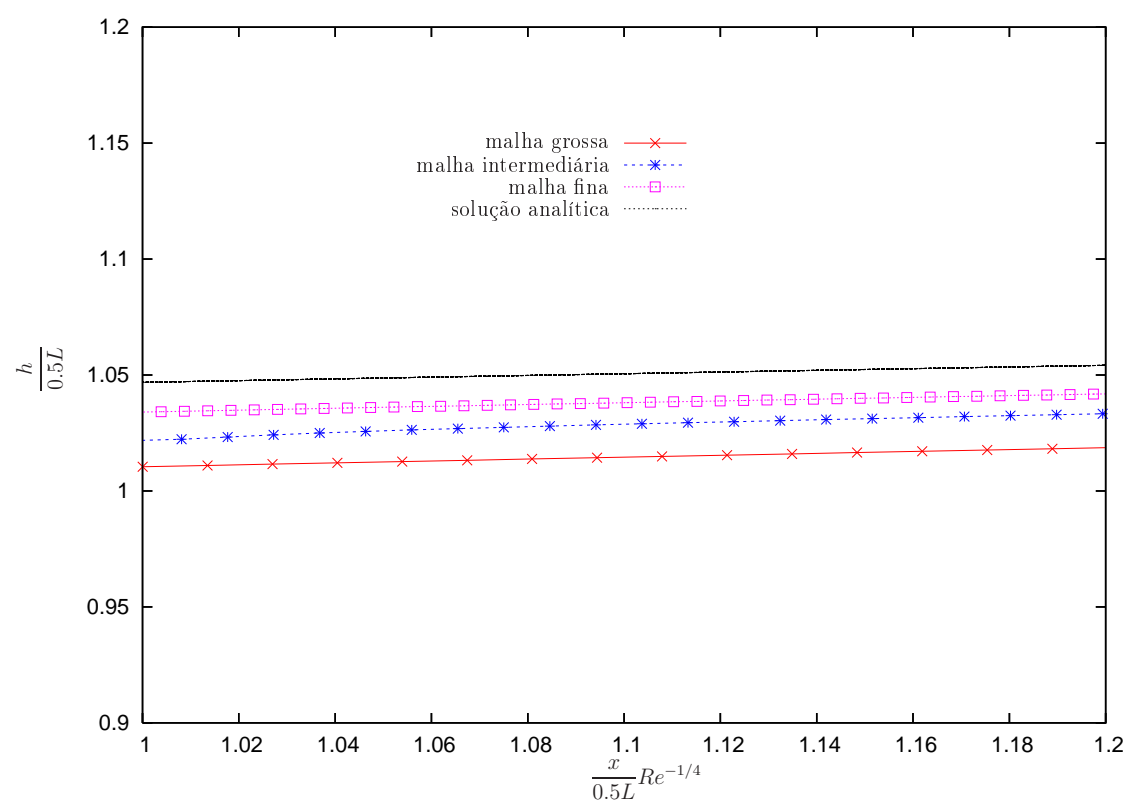

Figura 5.64: Ampliação das soluções numéricas e da solução analítica de Watson.

A Figura 5.63 e sua ampliação na Figura 5.64 mostram a comparação entre a altura da 
superfície livre computada pelo código Freeflow $2 D$ atual nas três malhas e a solução analítica de Watson no tempo $t=1.0 \mathrm{~s}$ (tempo em que o escoamento atinge o estado estacionário). Pode-se observar a partir dessa figura que os resultados numéricos nas três malhas estão em boa concordância com a solução analítica, embora, indicando pequenas diferenças em algumas regiões. Como no caso laminar (ver subseção 5.2.2), essas diferenças podem ser atribuídas ao número insuficiente de pontos junto a parede rígida. Observa-se ainda, dessa mesma figura, que a medida que se refina a malha a solução numérica converge para uma solução muito próxima da solução analítica.

A Figura 5.65 ilustra a simulação do problema no tempo $t=1.0 \mathrm{~s}$ para os campo de pressão e de velocidade nas direções $x$ e $y$ na malha intermediária utilizando o esquema ADBQUICKEST.

\section{Campo de pressão}

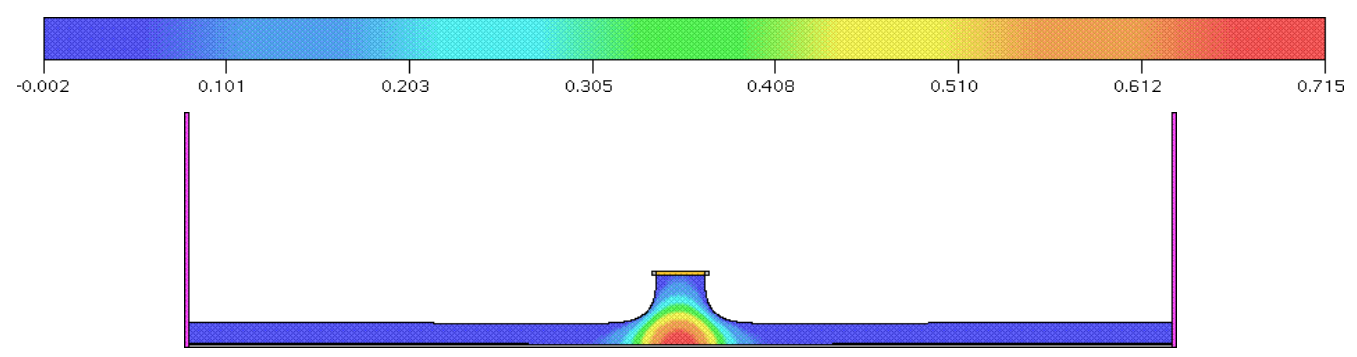

Campo de velocidade na direção $x$

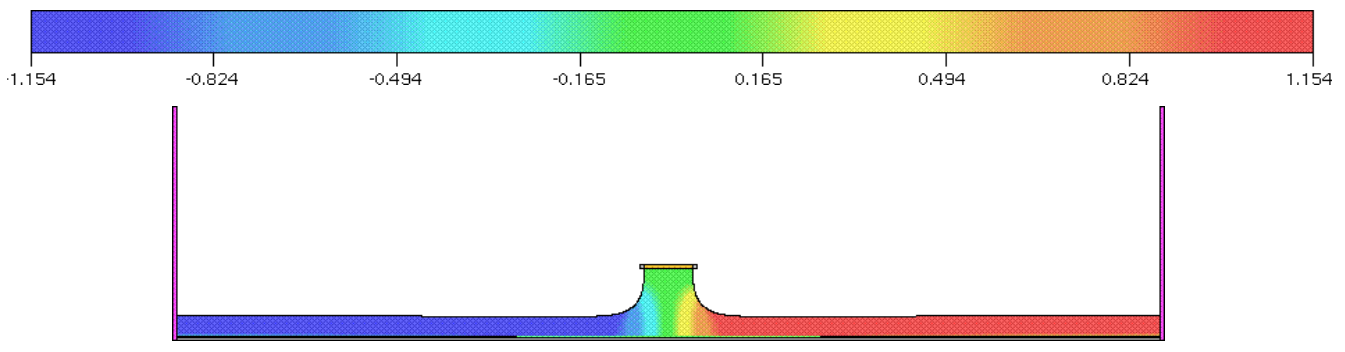

Campo de velocidade na direção $y$

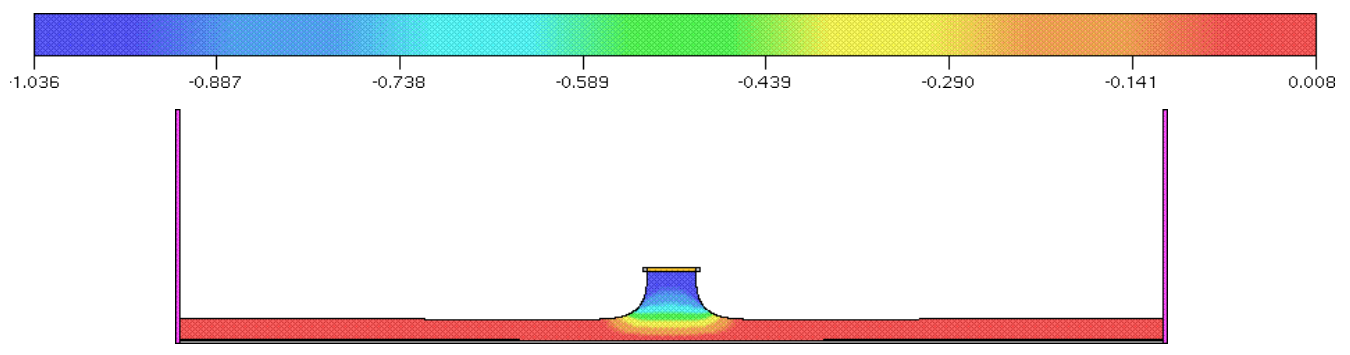

Figura 5.65: Jato livre 2D no regime turbulento utilizando $R e=5.0 \times 10^{4}$. 


\subsubsection{Aplicações 2D}

\subsubsection{Jato planar numa porção de fluido em repouso}

Nesta subseção são apresentados os resultados da simulação numérica de um jato horizontal turbulento, a uma distância $H=6.0 \mathrm{~m}$ de uma superfície livre, penetrando em uma porção de fluido em repouso (ver Figura 5.66). O objetivo nesta simulação é mostrar que o código FreeFlow $2 D$ incrementado com o ADBQUICKEST e o modelo $\kappa-\varepsilon$ é capaz de simular estruturas coerentes e suas interações com uma superfície livre. O diagrama esquemático desse problema com superfície livre e os parâmetros empregados na simulação são mostrados na Figura 5.66. $\mathrm{Na}$ simulação, os números de Reynolds e de Froude assumiram os valores $R e=U D / \nu=$ $5.0 \times 10^{4}$ e $F r=U / \sqrt{g D} \approx 12.77$, respectivamente. A malha utilizada foi de $100 \times 100$ células computacionais $(\delta x=\delta y=0.010 \mathrm{~m})$.

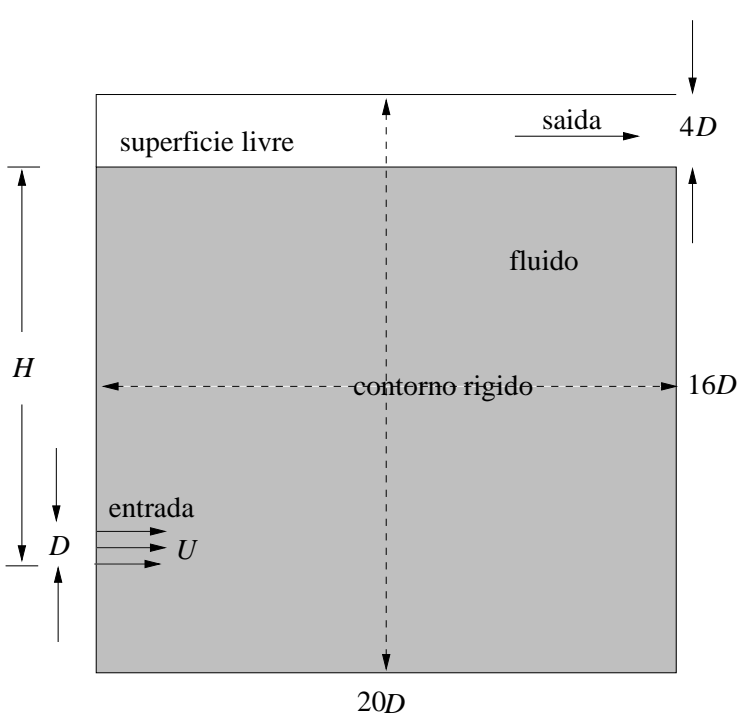

Figura 5.66: Geometria e parâmetros para o problema de um jato horizontal turbulento penetrando em uma porção de fluido estacionário: $U=2.0 \mathrm{~ms}^{-1}$ e $D=0.05 \mathrm{~m}$.

O desenvolvimento do campo de pressão e as distribuições das componentes $u$ e $v$ de velocidade, juntamente com a superfície livre, em vários tempos estão ilustrados nas Figuras 5.67, 5.68 e 5.69. Pode-se observar a partir dessas figuras a formação de um par de vórtices com orientações contrárias no início da simulação. Nota-se ainda, na região próxima a entrada do jato, outras estruturas maiores. No tempo final de simulação $t=5.28 \mathrm{~s}$, observa-se a presença de estruturas turbilhonares por todo o campo do escoamento. Essa simulação a altos números de Reynolds e de Froude fornece resultados consistentes com os encontradas na literatura (ver, por exemplo, Walker et al. [151] e Ferreira et al. [42]). É importante observar que, muito embora esta simulação seja feita em duas dimensões, pode-se considerar que ela assemelha-se à uma simulação com LES. Simulações de escoamentos turbulentos usando a metodologia LES e DNS têm sido realizadas por outros autores, mas estas são restritas a baixos números de Froude (ver Mangiavacchi et al. [91] e Shen e Yue [118]). 

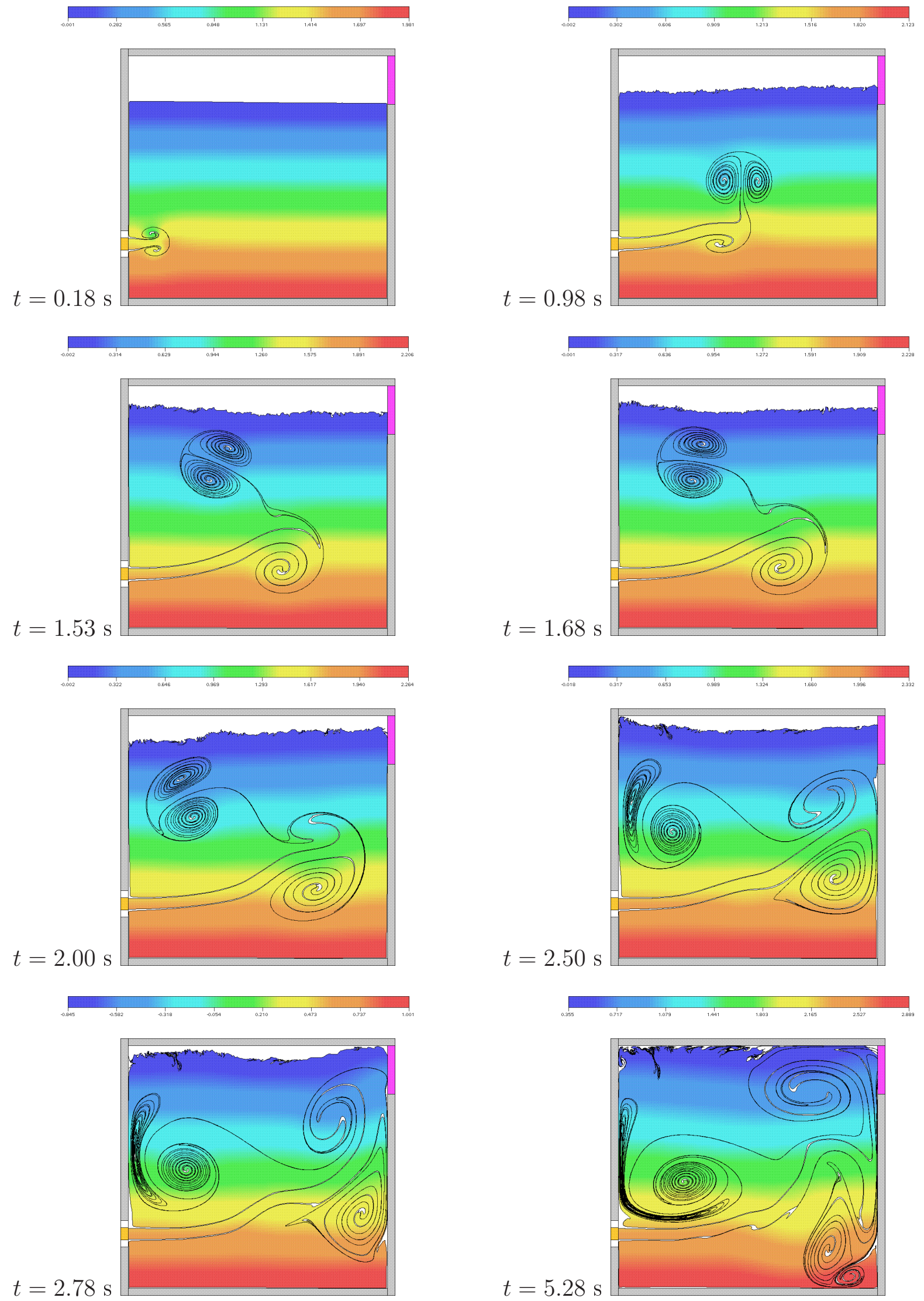

Figura 5.67: Evolução dos contornos de pressão de um jato penetrando em uma porção de fluido. 

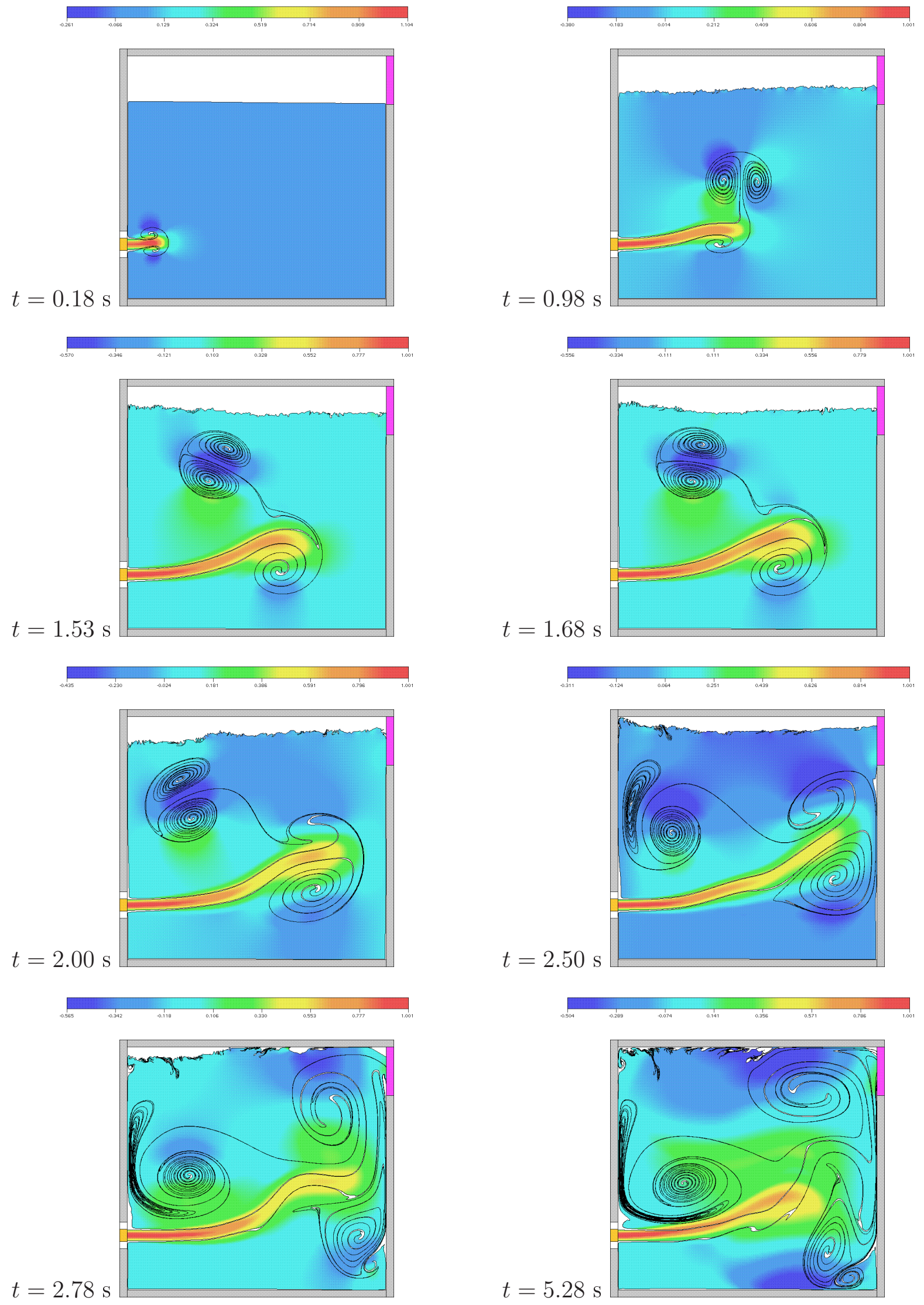

Figura 5.68: Evolução dos contornos de velocidade $u$ de um jato penetrando em uma porção de fluido. 

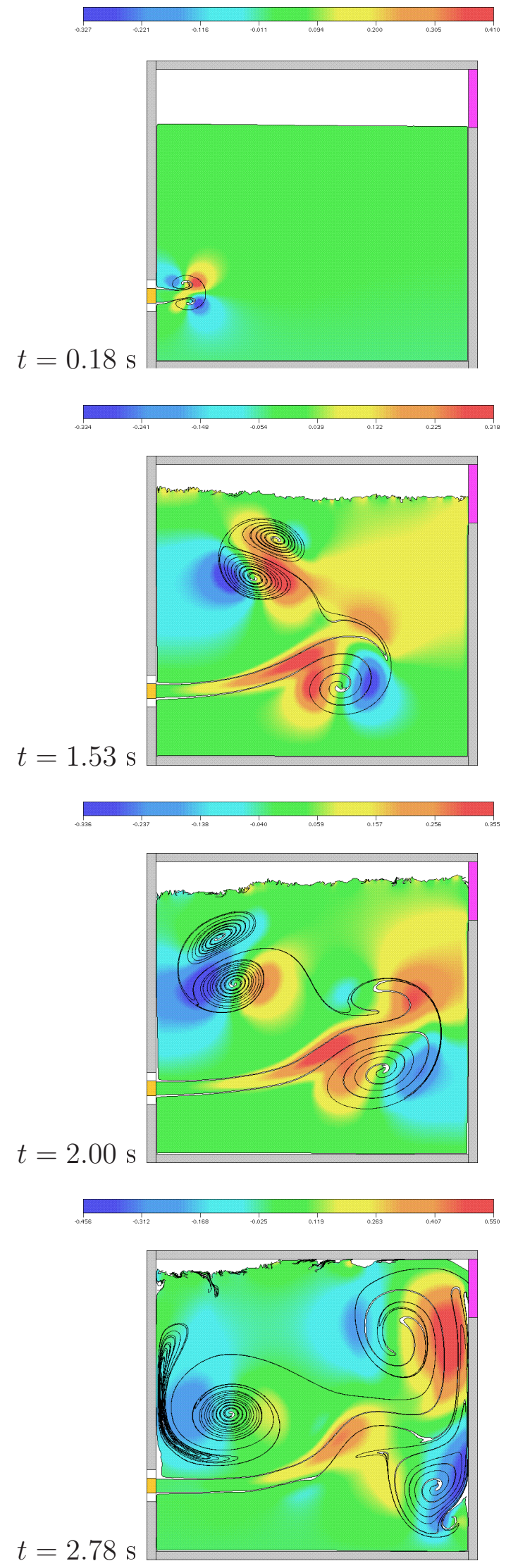
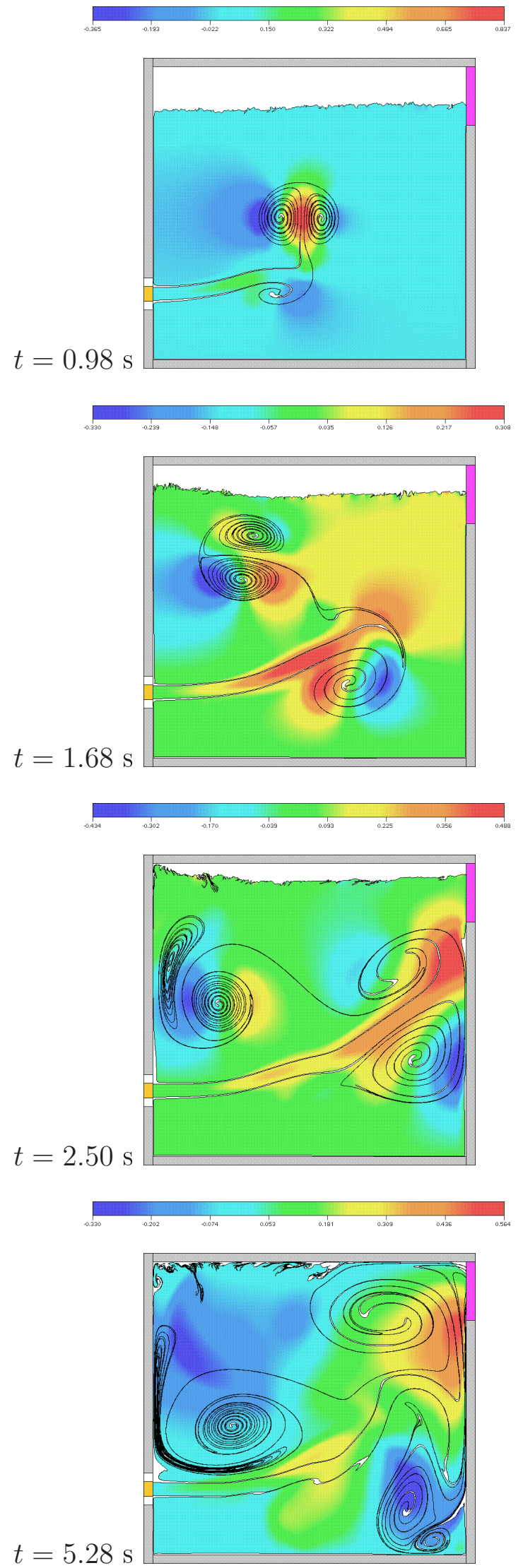

Figura 5.69: Evolução dos contornos de velocidade $v$ de um jato penetrando em uma porção de fluido. 


\subsubsection{Problema "sluice gate"}

Esta subseção é finalizada apresentando a simulação numérica de um outro tipo de colapso de uma coluna de fluido (Harlow e Welch [53]) que corresponde a um problema conhecido na literatura como "sluice gate". Este problema é geralmente utilizado como um modelo para investigar escoamentos em estruturas hidráulicas, onde os efeitos da superfície livre, das flutuações de turbulência e da distribuição de pressão são frequentemente requeridos (ver, por exemplo, Mouaze et al. [97], Sankaranarayanan e Suresh Rao [117]). Os parâmetros utilizados nessa simulação são as mesmos daqueles utilizados no problema do colapso 2D simulado anteriormente. O desenvolvimento do campo de pressão mais a evolução da superfície livre em diferentes tempos estão ilustrados na Figura 5.70. Pode-se observar a partir dessa figura, uma visível formação das estruturas coerentes por todo o escoamento. Em particular, esta figura representa a interação dessas grandes estruturas com a superfície livre.

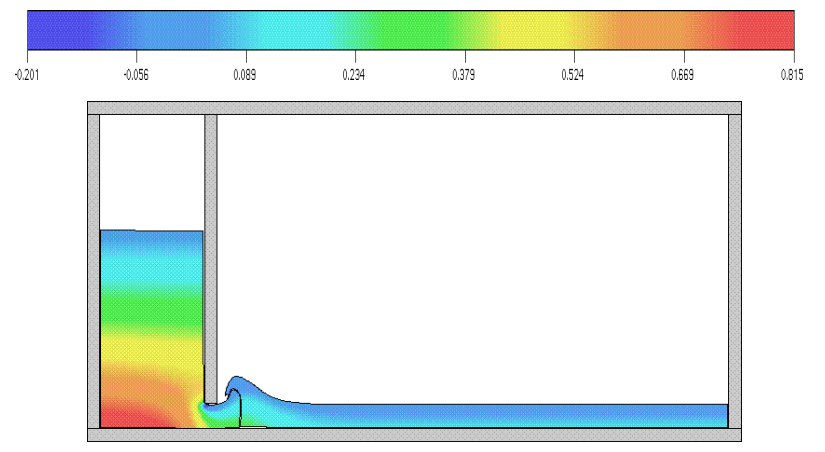

$t=0.50 \mathrm{~s}$

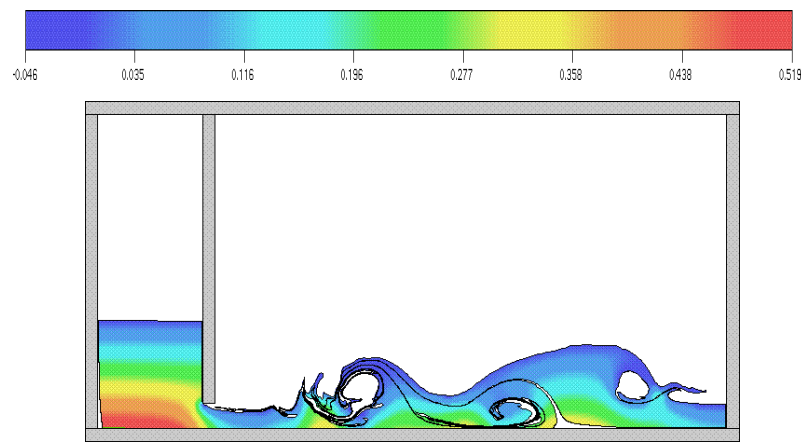

$t=3.5 \mathrm{~s}$
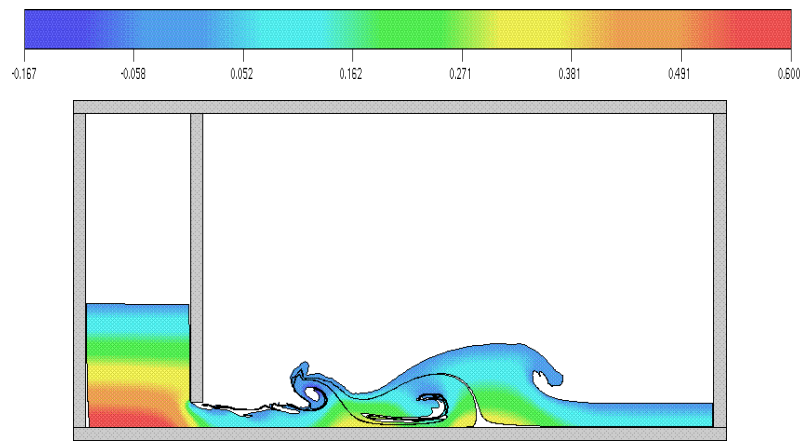

$t=2.5 \mathrm{~s}$

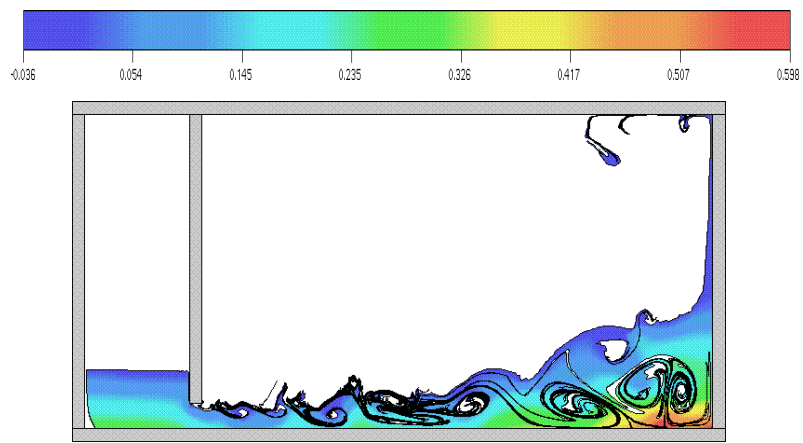

$t=5.0 \mathrm{~s}$

Figura 5.70: Desenvolvimento do campo de pressão e a evolução do perfil da superfície livre da simulação numérica 2D do problema "sluice gate". 
Neste ponto, são apresentados resultados numéricos de problemas 3D no regime turbulento e com superfícies livres móveis. E para a simulação desses problemas altamente complexos, o código Freeflow $3 D$ foi atualizado com o esquema "upwind" de alta resolução ADBQUICKEST e o modelo $\kappa-\varepsilon$ de turbulência. Validação, verificação e aplicações são também fornecidas.

\subsubsection{Colapso de um bloco de fluido}

O problema do colapso 3D é novamente considerado aqui para validar o método numérico. Vale lembrar que este problema foi estudado experimentalmente por Martin e Moyce [93] e Koshizuka e Oka [70], e mais recentemente Violeau e Issa [150] investigaram o desempenho do método SPH ("Smoothed Particle Hydrodynamics") nesse problema.

De maneira análoga ao caso laminar (ver subseção 5.2.3), considera-se um bloco de fluido com $a=0.05 \mathrm{~m}$ de comprimento, $b=2 a$ largura, $c=2 a$ de altura em equilíbrio hidrostático confinado entre paredes fixas (ver Figura 5.71). Em $t=0 \mathrm{~s}$, o bloco é instantaneamente posto à ação da gravidade e, consequentemente, sofre colapso. A fim de comparar os resultados numéricos com os dados experimentais de Martin e Moyce [93] e de Koshizuka e Oka [70], a condição "free-slip" foi implementada.

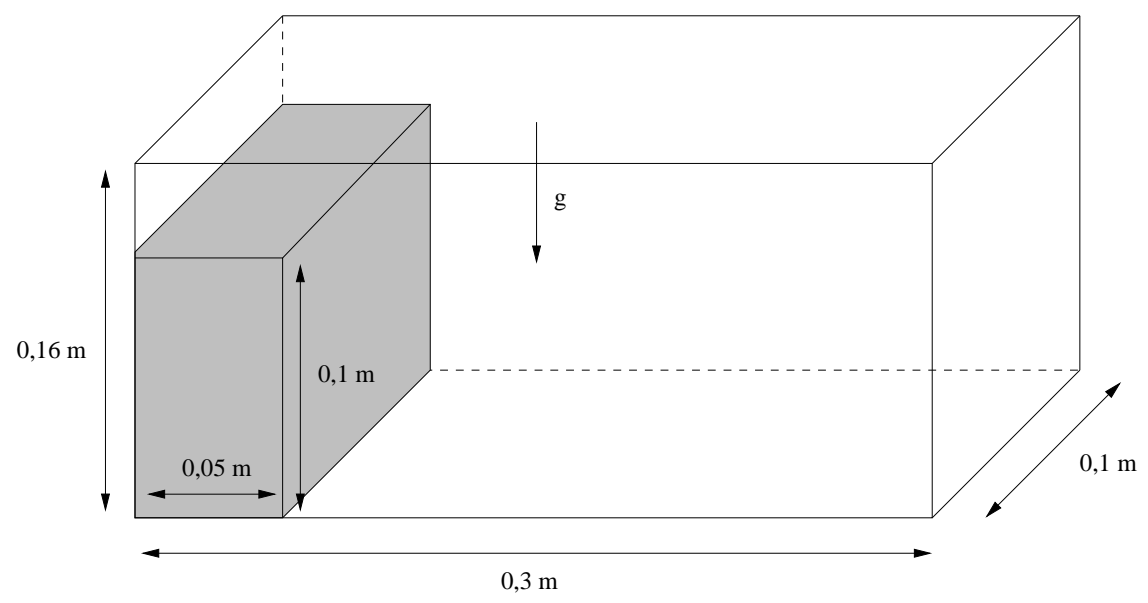

Figura 5.71: Geometria do problema do colapso de um bloco de fluido.

Para a simulação do problema, os seguintes dados foram empregados:

- Dimensão do domínio: $0.3 \mathrm{~m} \times 0.1 \times 0.16 \mathrm{~m}$;

- Constante gravitacional: $g=9.81 \mathrm{~ms}^{-2}$;

- Coeficiente de viscosidade cinemática: $\nu=1.0 \times 10^{-6} \mathrm{~m}^{2} \mathrm{~s}^{-1}$;

- Parâmetros de escala: $L=0.1 \mathrm{~m}$ e $U=\sqrt{L g}=0.99045444 \mathrm{~ms}^{-1}$;

- Número de Reynolds: $R e=U L / \nu=99.045,444$;

- Número de Froude: $\operatorname{Fr}=U / \sqrt{g L}=1.0$. 
Quatro malhas $(60 \times 20 \times 32(\delta x=\delta y=0.005 \mathrm{~m}), 75 \times 25 \times 40(\delta x=\delta y=0.004 \mathrm{~m})$, $150 \times 50 \times 80(\delta x=\delta y=0.0002 \mathrm{~m})$ e $300 \times 100 \times 260(\delta x=\delta y=0.001 \mathrm{~m}))$ foram utilizadas nas simulações. O número de Reynolds crítico $y_{c}^{+}$e as constantes $K$ e $B$, nas leis de parede, assumiram os seguintes valores: $y_{c}^{+} \approx 10,87, K=0.41$ e $B=5.0$, respectivamente. O critério de convergência para a equação de Poisson foi $1.0 \times 10^{-12}$ e o tamanho do passo temporal $1.056 \times 10^{-6}$.

Um teste de validação é apresentada na Figura 5.72 para este problema. Essa figura mostra a comparação dos dados experimentais de Koshizuka e Oka [70] e de Martin e Moyce [93] com os resultados numéricos nas quatro malhas, da posição frontal do movimento do fluido $\left(X_{\max }\right)$ versus o tempo. A solução numérica foi computada do corte do plano $x z$ na posição $y=0.05$ m. Pode-se observar nessa figura que os resultados numéricos estão em boa concordância com os dados experimentais, proporcionando confiabilidade do código Freeflow 3D, adaptado com as modelagens ADBQUICKEST e $\kappa-\varepsilon$, em resolver escoamentos turbulentos com superfícies livres. Observa-se, ainda, por essa mesma figura que, ao se refinar a malha, a solução numérica converge para uma solução próxima dos dados experimentais.

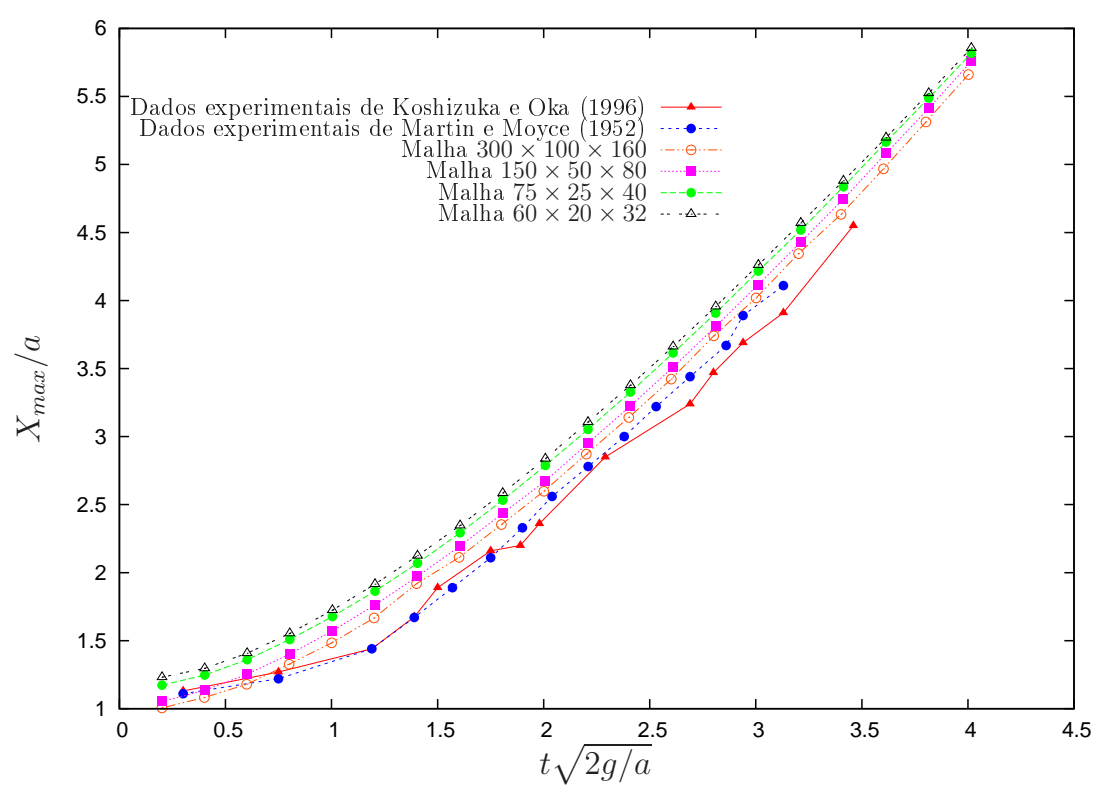

Figura 5.72: Comparação entre os dados experimentais de Martin e Moyce e Koshizuka e Oka e as soluções numéricas $3 \mathrm{D}$ nas quatro malhas da posição frontal do movimento do fluido $\left(X_{\max }\right)$.

A Figura 5.73 mostra a comparação dos dados experimentais com os resultados numéricos obtidos nos casos: (i) usando a modelagem ADBQUICKEST; e (ii) usando ambas as modelagens ADBQUICKEST e $\kappa-\varepsilon$. Observa-se nessa figura que a solução numérica obtida no caso (ii) (isto é, com o modelo $\kappa-\varepsilon$ ) forneceu os melhores resultados.

A Figura 5.74 mostra a simulação numérica desse problema com superfície livre 3D, obtida pelo código Freeflow $3 D$ equipado com as modelagens ADBQUICKEST e $\kappa-\varepsilon$, nos tempos $t=0.0 \mathrm{~s}, t=1.0 \mathrm{~s}, t=1.5 \mathrm{~s}$ e $t=2.0 \mathrm{~s}$. E as Figuras $5.75,5.76$ e 5.77 mostram, respectivamente, 


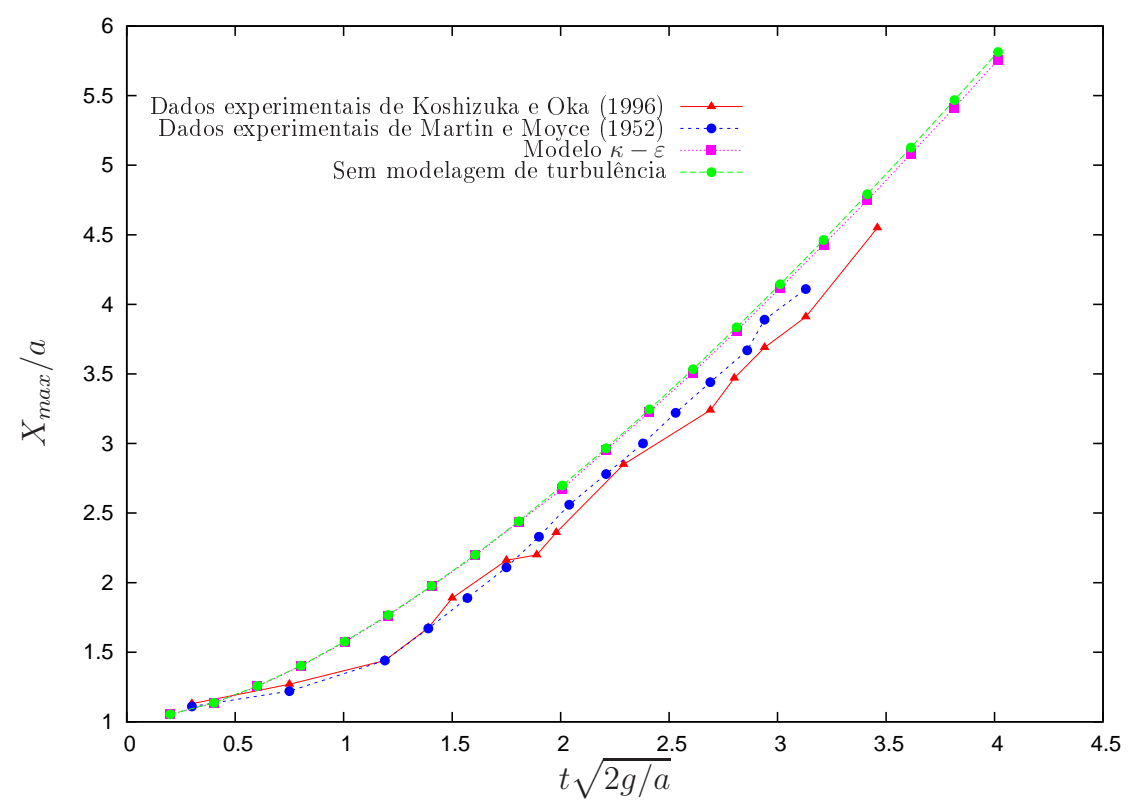

Figura 5.73: Comparação entre os os dados experimentais e os resultados numéricos obtidos sem modelagem de turbulência e com o modelo $\kappa-\varepsilon$.

os contornos de pressão e de velocidade nas direções $x$ e $z$ na malha $150 \times 50 \times 80$ nos tempos $t=0.5 \mathrm{~s}, t=1.0 \mathrm{~s}, t=1.5 \mathrm{~s}$ e $t=2.0 \mathrm{~s}$.
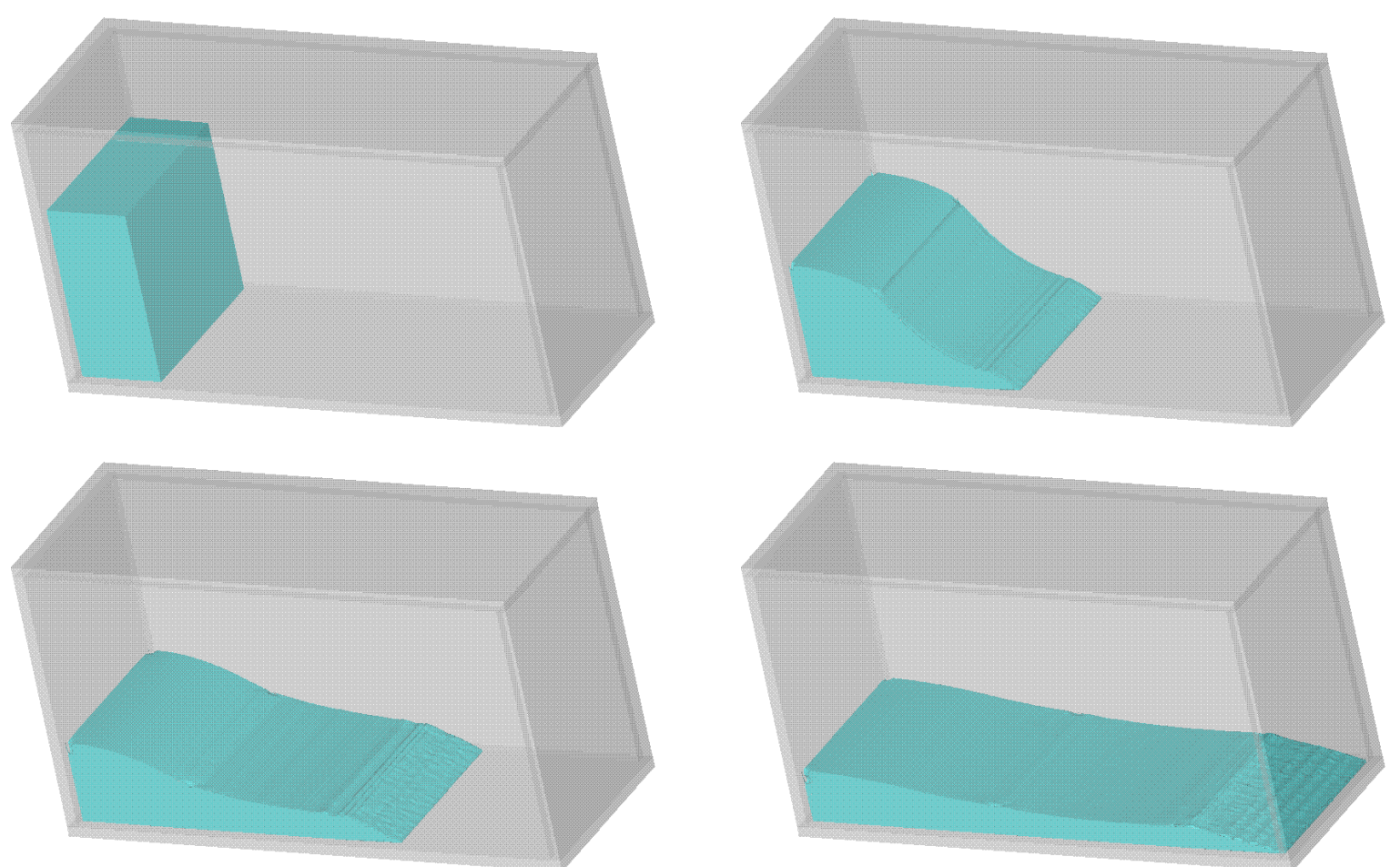

Figura 5.74: Simulação numérica 3D mostrando a evolução da superfície livre em diferentes tempos. 

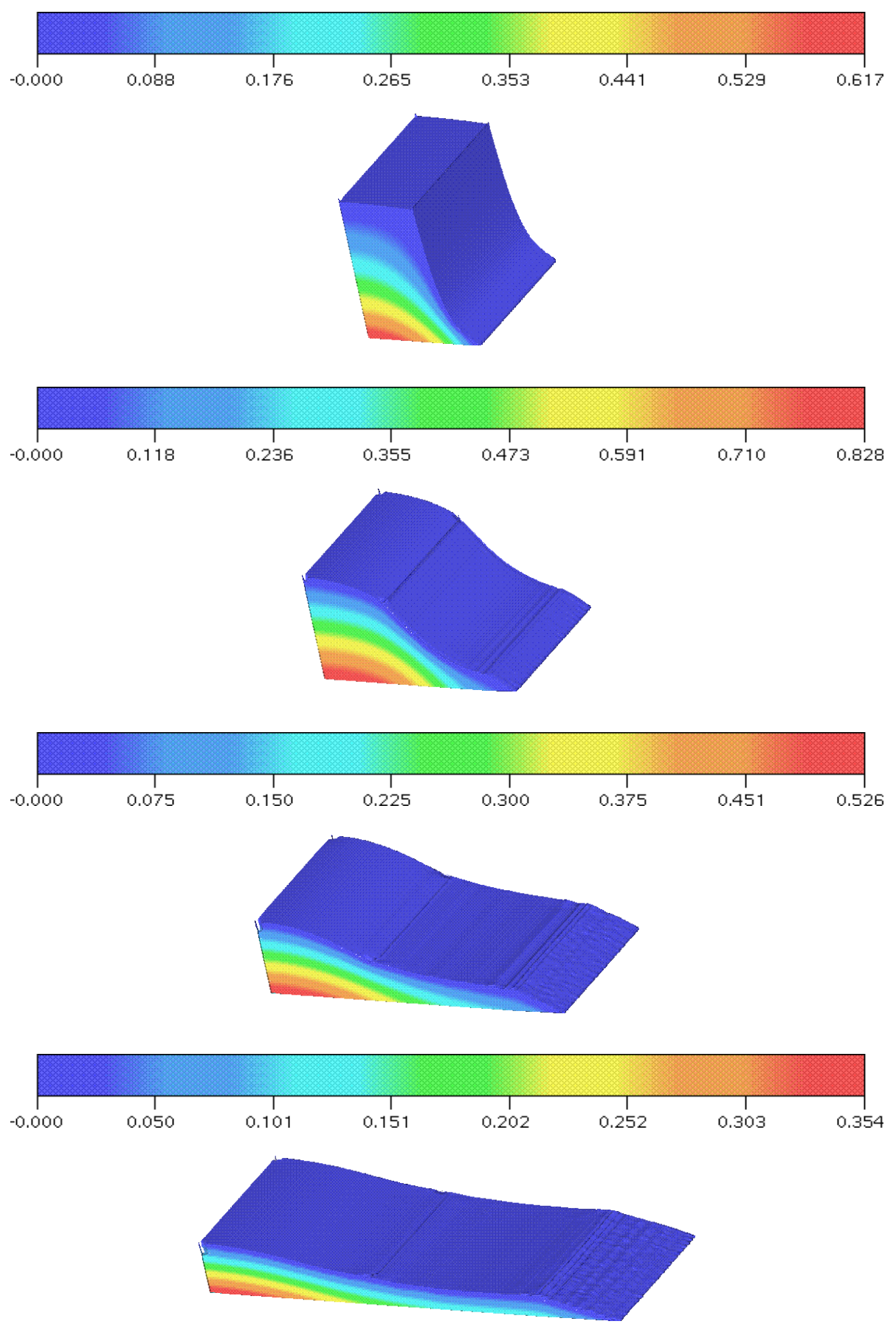

Figura 5.75: Simulação numérica 3D mostrando o contorno da pressão em diferentes tempos. 

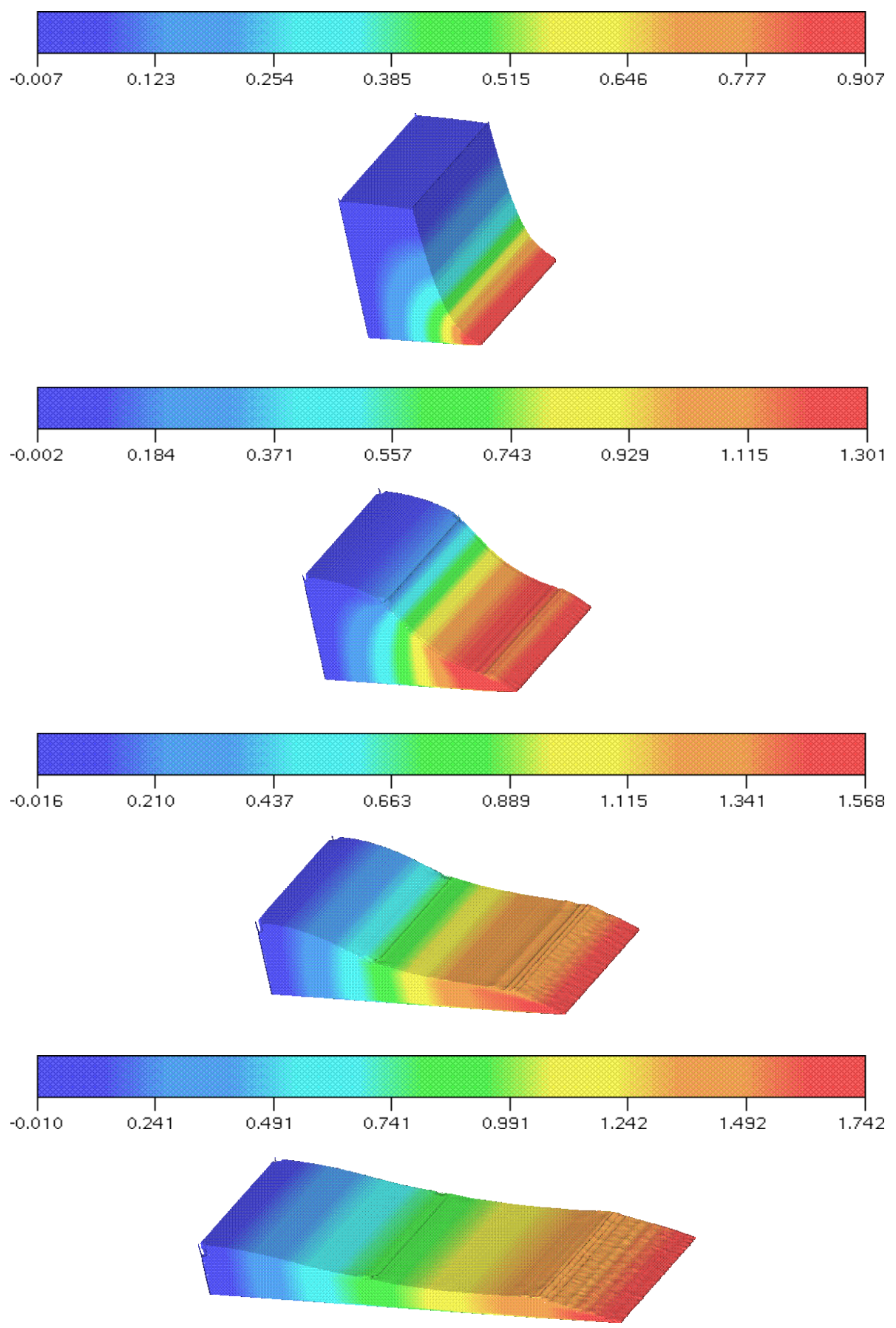

Figura 5.76: Simulação numérica 3D mostrando o contorno da velocidade na direção $x$ em diferentes tempos. 

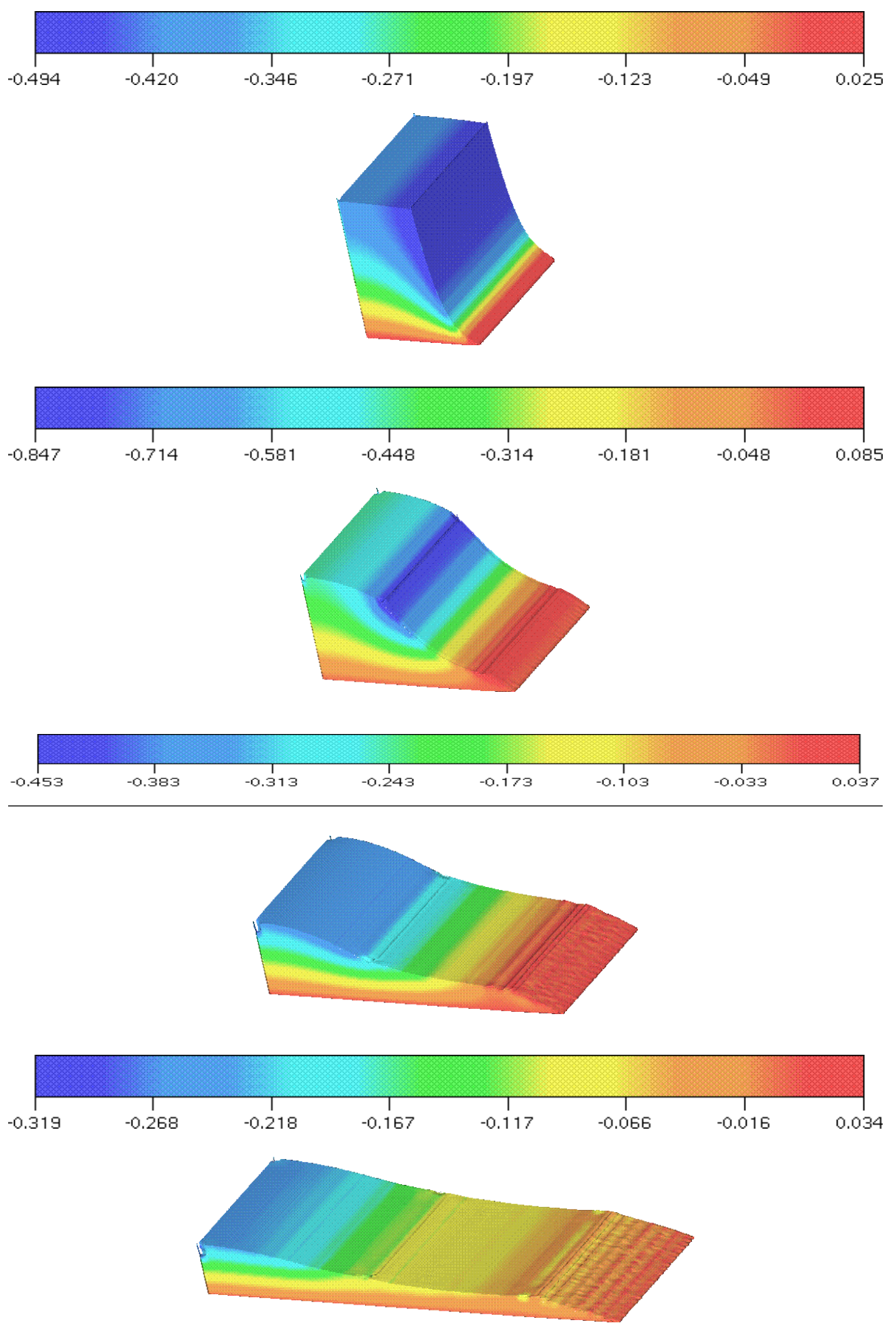

Figura 5.77: Simulação numérica 3D mostrando o contorno da velocidade na direção $z$ em diferentes tempos. 


\subsubsection{Jato livre sobre uma superfície rígida impermeável}

Nesta subseção é apresentada uma comparação entre a solução numérica obtida com o código Freeflow 3D equipado com as modelagens ADBQUICKEST e $\kappa-\varepsilon$ e as soluções analíticas (viscosa e não viscosa) de Watson [155]. O problema em questão é o de um jato livre sobre uma superfície rígida impermeável e posterior espalhamento nas direções radiais, cuja geometria 2D e os parâmetro de simulação estão descritos na Figura 5.78.

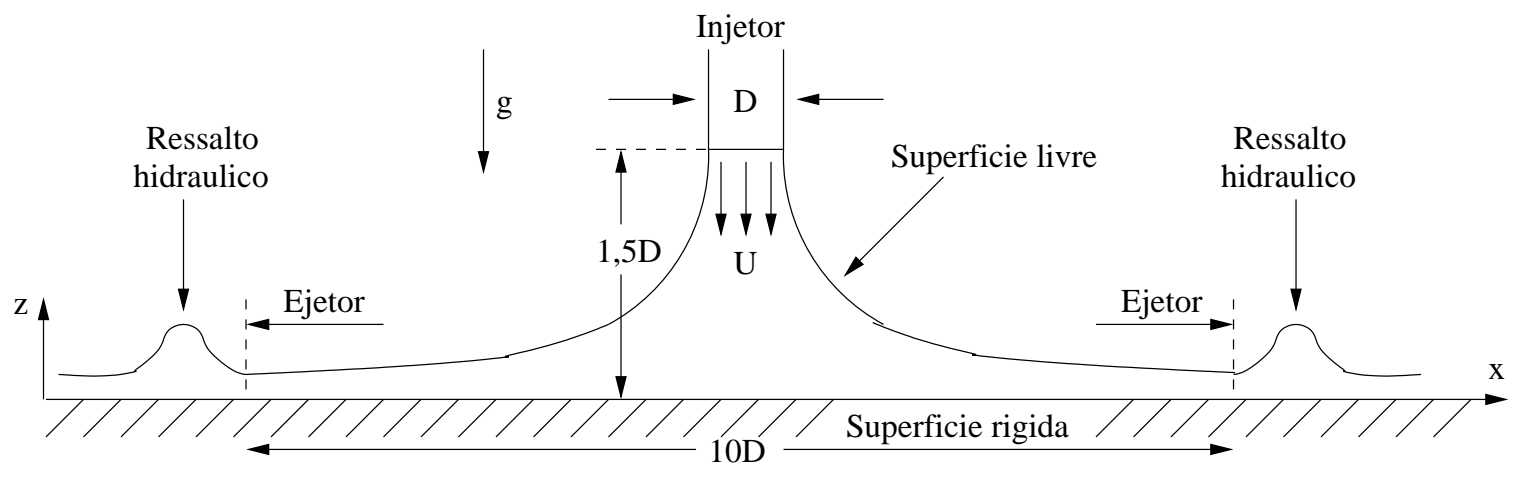

Figura 5.78: Configuração esquemática 2D mostrando a geometria e os parâmetros: $U=2.0$ $\mathrm{ms}^{-1}$ e $D=0.01 \mathrm{~m}$.

Em resumo, a solução analítica de Watson para este caso de escoamento turbulento é dada como segue.

$$
h(x)= \begin{cases}\frac{9 k}{200}(14 \pi A)^{1 / 4}\left(\frac{x^{9 / 4}+l^{9 / 4}}{x}\right), & \text { se } x \geq x_{0}, \\ \frac{1}{2 x}+\left(\frac{k-A}{k}\right) \delta, & \text { se } x<x_{0},\end{cases}
$$

em que $k=0.260, A=0.239 \mathrm{e}$

$$
\delta=\left[80\left(A-\frac{2}{9}\right)\right]^{-4 / 5} k(7 \pi)^{1 / 5} x^{4 / 5} .
$$

A constante $x_{0}$ foi derivada por Watson como

$$
x_{0}=(7 \pi)^{-1 / 9}(2 A)^{-5 / 9}\left[80\left(A-\frac{2}{9}\right)\right]^{4 / 9} .
$$

E, a constante $l$ que aparece em (5.29) foi deduzida por Watson como sendo

$$
l=20(1-2 A)(14 \pi)^{-1 / 4} A^{-5 / 4} .
$$

Para a simulação do problema com superfície livre em questão, o seguinte modelo foi adotado: 
- Dimensão do domínio: $0.1 \mathrm{~m} \times 0.1 \mathrm{~m} \times 0.02 \mathrm{~m}$;

- Diâmetro do injetor: $D=0.01 \mathrm{~m}$;

- Velocidade de injeção: $U=2.0 \mathrm{~ms}^{-1}$;

- Constante gravitacional: $g=9.81 \mathrm{~ms}^{-2}$;

- Coeficiente de viscosidade cinemática: $\nu=6.25 \times 10^{-7} \mathrm{~m}^{2} \mathrm{~s}^{-1}$;

- Parâmetros de escala: $D=0.01 \mathrm{~m} \mathrm{e} U=2.0 \mathrm{~ms}^{-1}$;

- Altura do injetor a partir da superfície rígida: $H=0.015 \mathrm{~m}$;

- Número de Reynolds: $R e=\frac{\pi}{2} U D / \nu=50000$;

- Número de Froude: $F r=U / \sqrt{g D}=2.019275$.

Três malhas (malha grossa $50 \times 50 \times 10-\delta x=\delta y=\delta z=0.002 \mathrm{~m}$; malha intermediária $100 \times 100 \times 20-\delta x=\delta y=\delta z=0.001 \mathrm{~m}$; e malha fina $200 \times 200 \times 40-\delta x=\delta y=\delta z=0.0005$ $\mathrm{m})$ e tempo final $t=4.0 \mathrm{~s}$ foram empregados na simulação. O número de Reynolds crítico $y_{c}^{+}$e as constantes $K$ e $B$ nas leis de parede assumiram os seguintes valores: $y_{c}^{+} \approx 11.048$, $K=0.41$ e $B=5.0$, respectivamente. O critério de convergência para a equação de Poisson foi $1.0 \times 10^{-12}$ e o tamanho do passo temporal $1.67 \times 10^{-5}$.

Testes de verificação do Freeflow 3D atualizado com o esquema ADBQUICKEST e modelo $\kappa-\varepsilon$ são apresentados nas Figuras 5.79 e 5.80. Em particular, na Figura 5.79 são apresentadas as soluções numéricas (nas três malhas) para a altura $h$ da superfície livre calculadas do corte do plano $x z$ na posição $y=0.05$, e na Figura 5.80 corresponde às soluções numéricas (nas três malhas) para a altura da superfície livre calculadas do corte do plano $y z$ na posição $x=0.05 \mathrm{~m}$. Pode-se observar a partir da Figura 5.79 (d) e da Figura 5.80 (d), que as soluções numéricas nas malhas grossas e intermediárias convergem para a solução da malha fina. Além disso, os resultados numéricos obtida na malha fina (Figura 5.79 (c) e Figura 5.80 (c)) mostram concordância com a solução analítica viscosa de Watson. As diferenças entre a solução analítica viscosa e a numérica em algumas regiões podem ser atribuídas ao número insuficiente de pontos da malha junto ao contorno rígido e as hipóteses adotadas por Watson (segundo ele, no caso turbulento, sua solução não concorda bem com dados experimentais). 
(a)

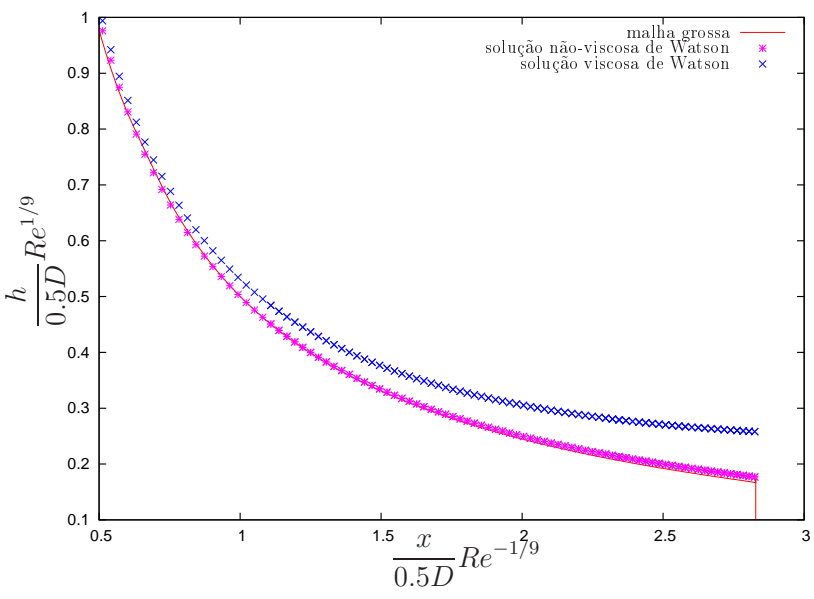

(c)

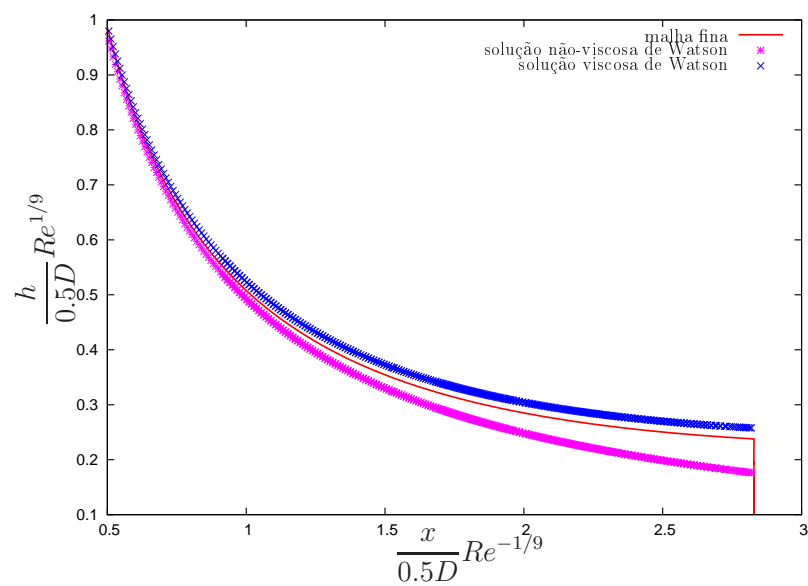

(b)

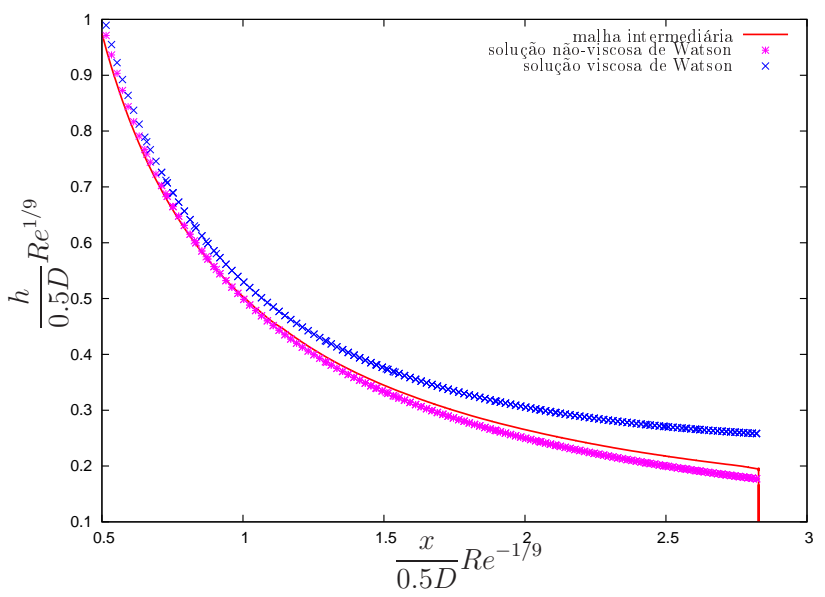

(d)

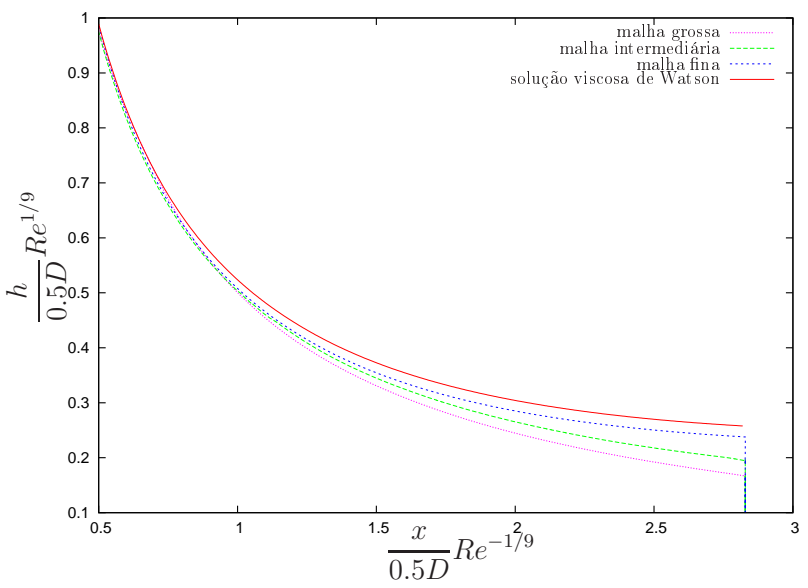

Figura 5.79: Soluções numéricas (corte do plano $x z$ ) obtida pelo código Freeflow 3D com modelo $\kappa-\varepsilon$ mostrando: (a) comparação na malha grossa; (b) comparação na malha intermediária; (c) comparação na malha fina; (d) comparação nas três malhas. 
(a)

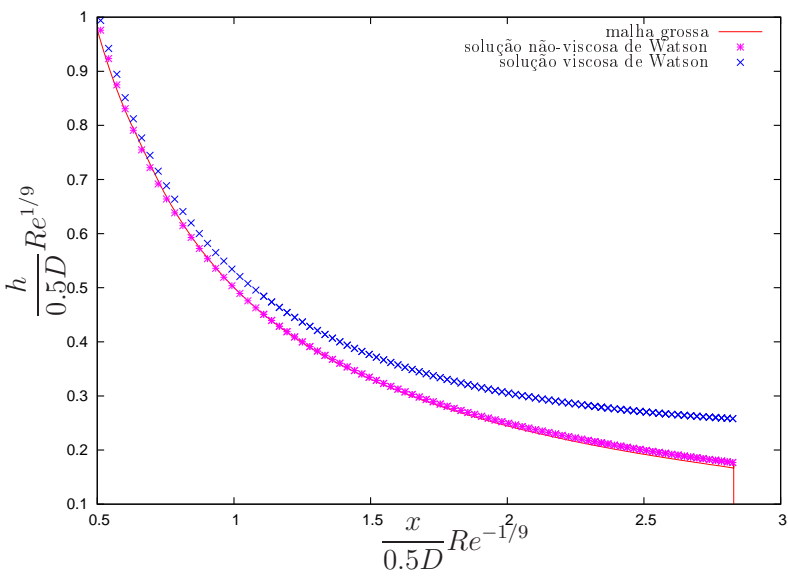

(c)

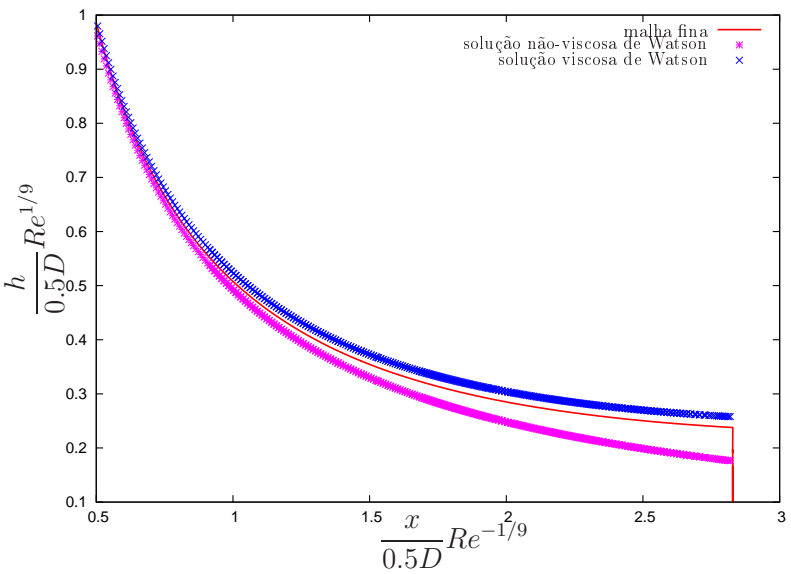

(b)

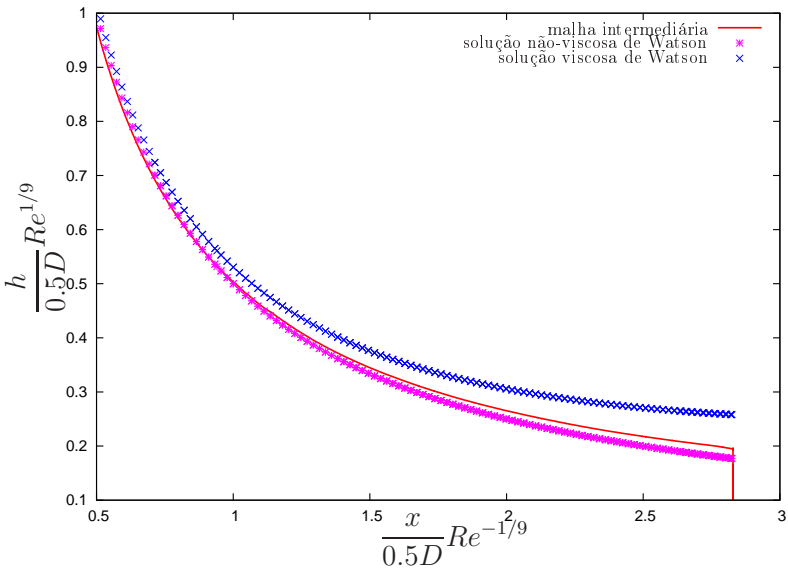

(d)

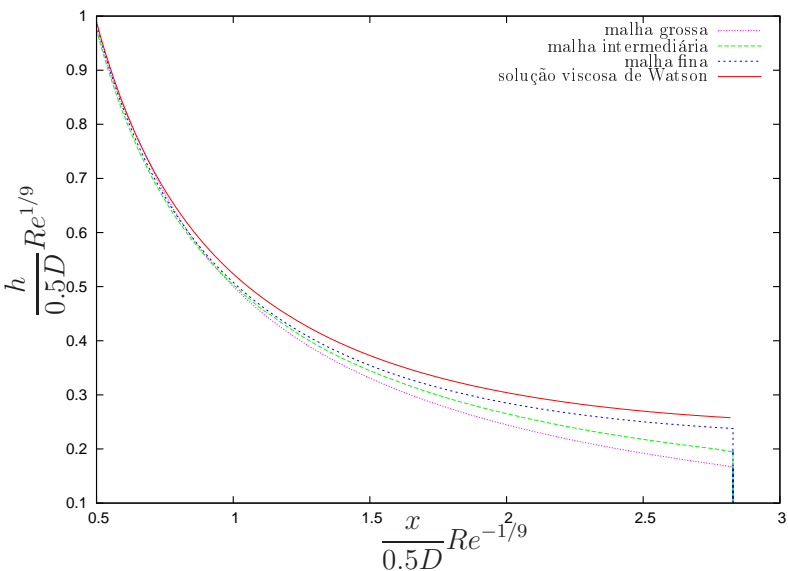

Figura 5.80: Soluções numéricas (corte do plano yz) obtida pelo código Freeflow 3D com modelo $\kappa-\varepsilon$ mostrando: (a) comparação na malha grossa; (b) comparação na malha intermediária; (c) comparação na malha fina; (d) comparação nas três malhas. 
A Figura 5.81 mostra a superfície livre calculada, com as modelagens investigadas nesse trabalho, na malha fina. Vale mencionar que o problema foi simulado antes que ocorresse o fenômeno do ressalto hidráulico.

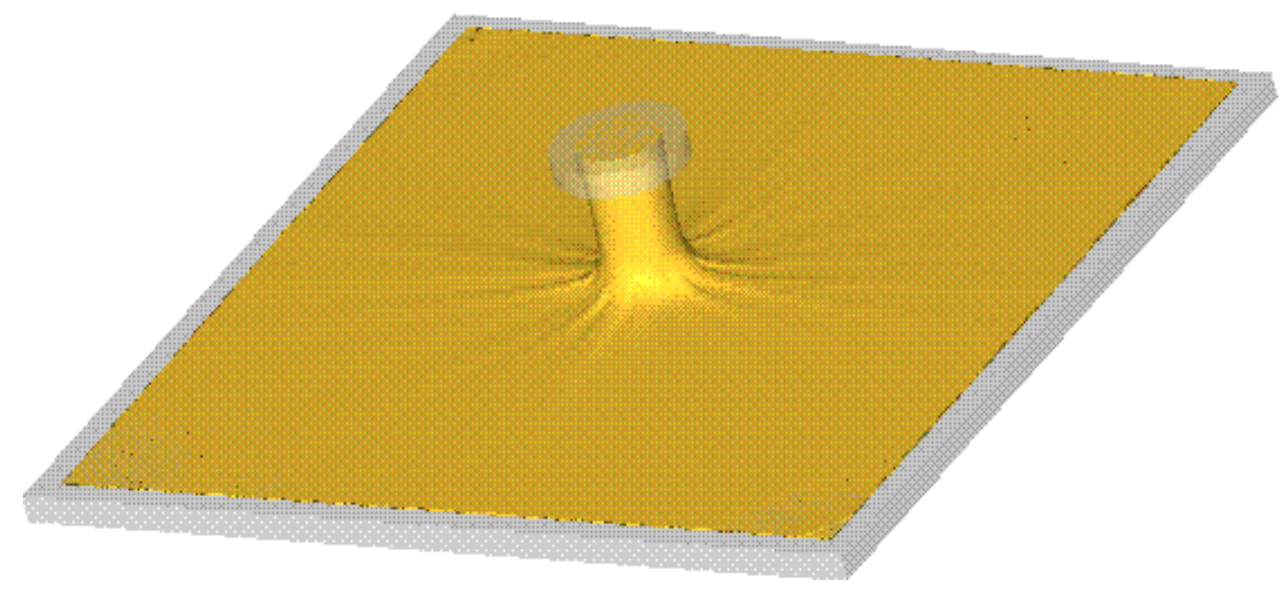

Figura 5.81: Simulação numérica 3D de um jato incidindo sobre uma superfície rígida.

As Figuras 5.82 e 5.83 mostram os cortes dos planos $x z$ e $y z$ nas posições $y=0.05$ e $x=0.05$, respectivamente, no tempo $t=4.0 \mathrm{~s}$, ilustrando os campos de pressão e velocidade.

\section{Campo de pressão}

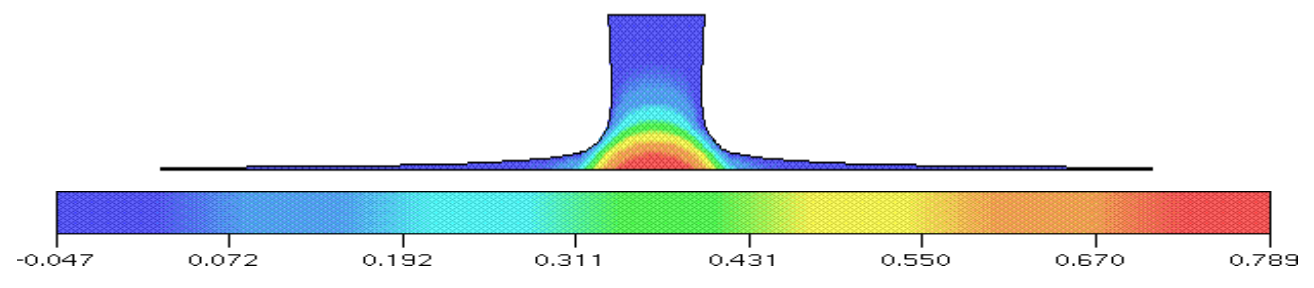

Campo de velocidade na direção $x$

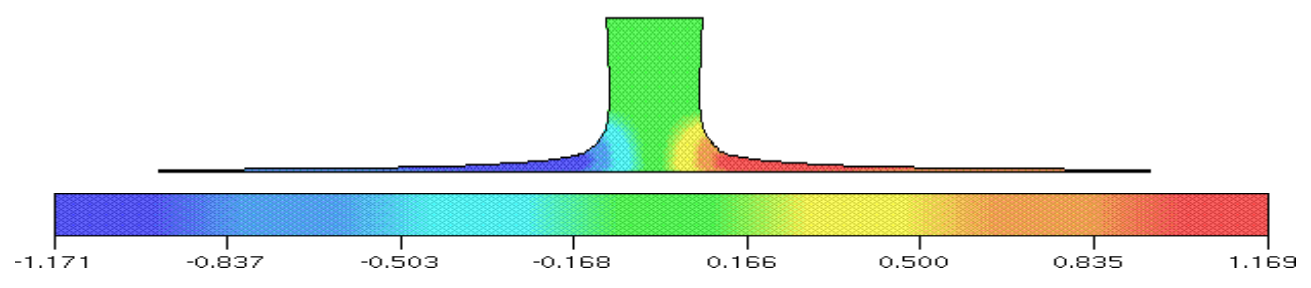

Campo de velocidade na direção $z$

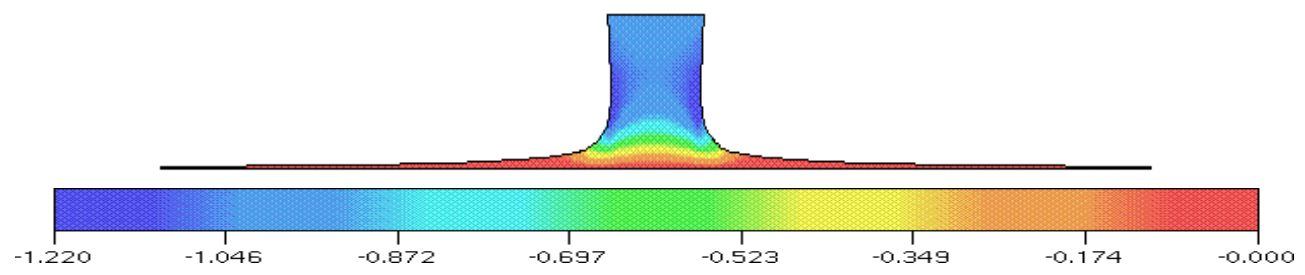

Figura 5.82: Visualizações do corte do plano $x z$ na posição $y=0.05$ da simulação numérica 3D do jato incidindo sobre uma superfície rígida. 


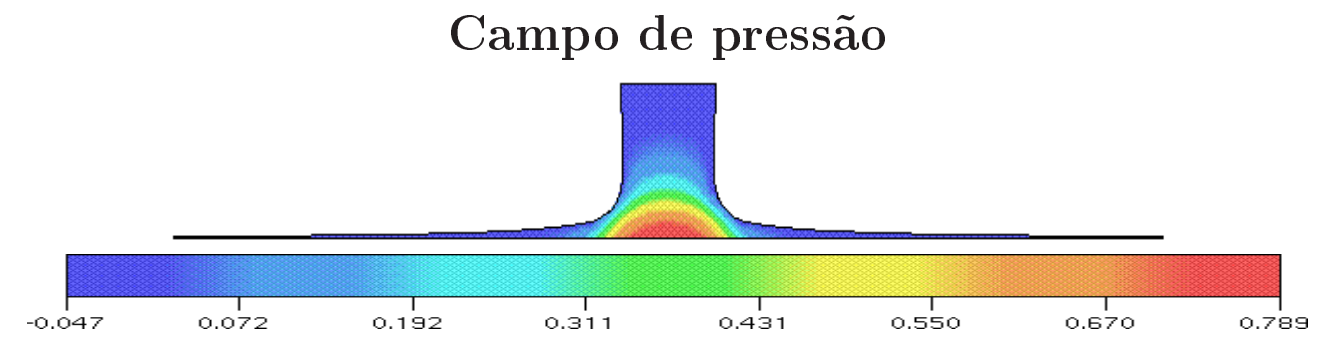

Campo de velocidade na direção y

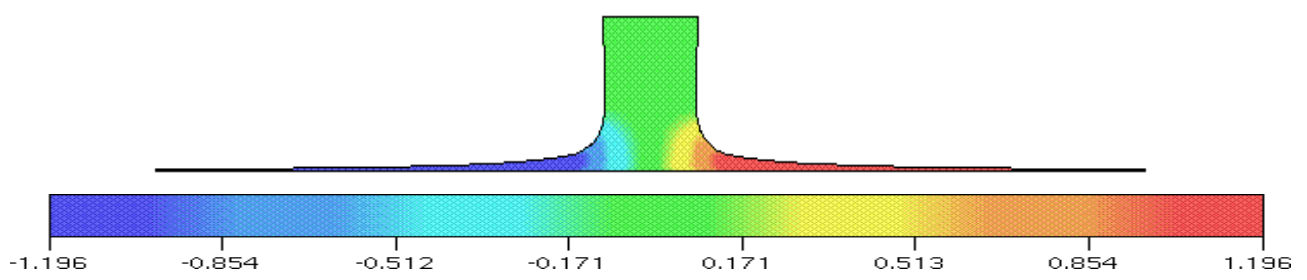

Campo de velocidade na direção $z$

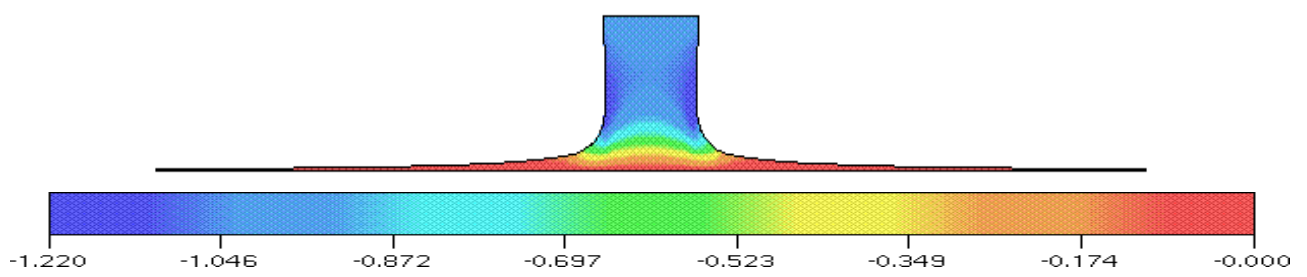

Figura 5.83: Visualizações do corte do plano $y z$ na posição $x=0.05$ da simulação numérica 3D do jato incidindo sobre uma superfície rígida no tempo. 


\subsubsection{Aplicações 3D}

Esta tese é finalizada apresentando mais simulações de problemas 3D com superfícies livres em regime turbulento utilizando o ambiente de simulação Freeflow $3 D$ equipado com as modelagens ADBQUICKEST e $\kappa-\varepsilon$.

\subsubsection{Experimento de Storr e Behnia}

Este problema, um experimento realizado por Storr e Behnia [129], consiste de um jato circular de água incidindo perpendicularmente sobre corpo de fluido (de mesma viscosidade do incidente) em repouso. Na simulação foram utilizadas uma malha de $50 \times 50 \times 50(\delta x=\delta y=$ $\delta z=0.002 \mathrm{~m}$ ) células computacionais, como critério de convergência na equação de Poisson a tolerância de $1.0 \times 10^{-8}$ e o tamanho do passo temporal de $4.167 \times 10^{-4}$. O número de Reynolds crítico $y_{c}^{+}$e as constantes $K$ e $B$ nas leis de parede assumiram os seguintes valores: $y_{c}^{+} \approx 11.048$, $K=0.41$ e $B=5.0$, respectivamente. Os demais dados para a simulação do problema estão descrito no modelo abaixo.

- Dimensão do domínio: $0.1 \mathrm{~m} \times 0.1 \times 01 \mathrm{~m}$;

- Diâmetro do injetor: $D=0.006 \mathrm{~m}$;

- Velocidade de injeção: $U=1.0 \mathrm{~ms}^{-1}$;

- Constante gravitacional: $g=9.81 \mathrm{~ms}^{-2}$;

- Coeficiente de viscosidade cinemática: $\nu=2.0 \times 10^{-6} \mathrm{~m}^{2} \mathrm{~s}^{-1}$;

- Parâmetros de escala: $D=0.006 \mathrm{~m} \mathrm{e} U=1.0 \mathrm{~ms}^{-1}$;

- Número de Reynolds: $R e=U D / \nu=3000$;

- Número de Froude: $F r=U / \sqrt{g D}=4.12183$;

- Altura do injetor a partir da base do container: $H=0.08 \mathrm{~m}$.

A Figura 5.84 mostra a comparação qualitativa entre o experimento realizado por Storr e Behnia e a simulação numérica no tempo final $t=6.0 \times 10^{-2} \mathrm{~s}$. As diferenças entre a simulação e o experimento podem ser atribuídas aos fatos de que o modelo utilizado para a simulação não ser fiel ao modelo físico adotado por Storr e Behnia e o código não levar em conta a tensão superficial. 

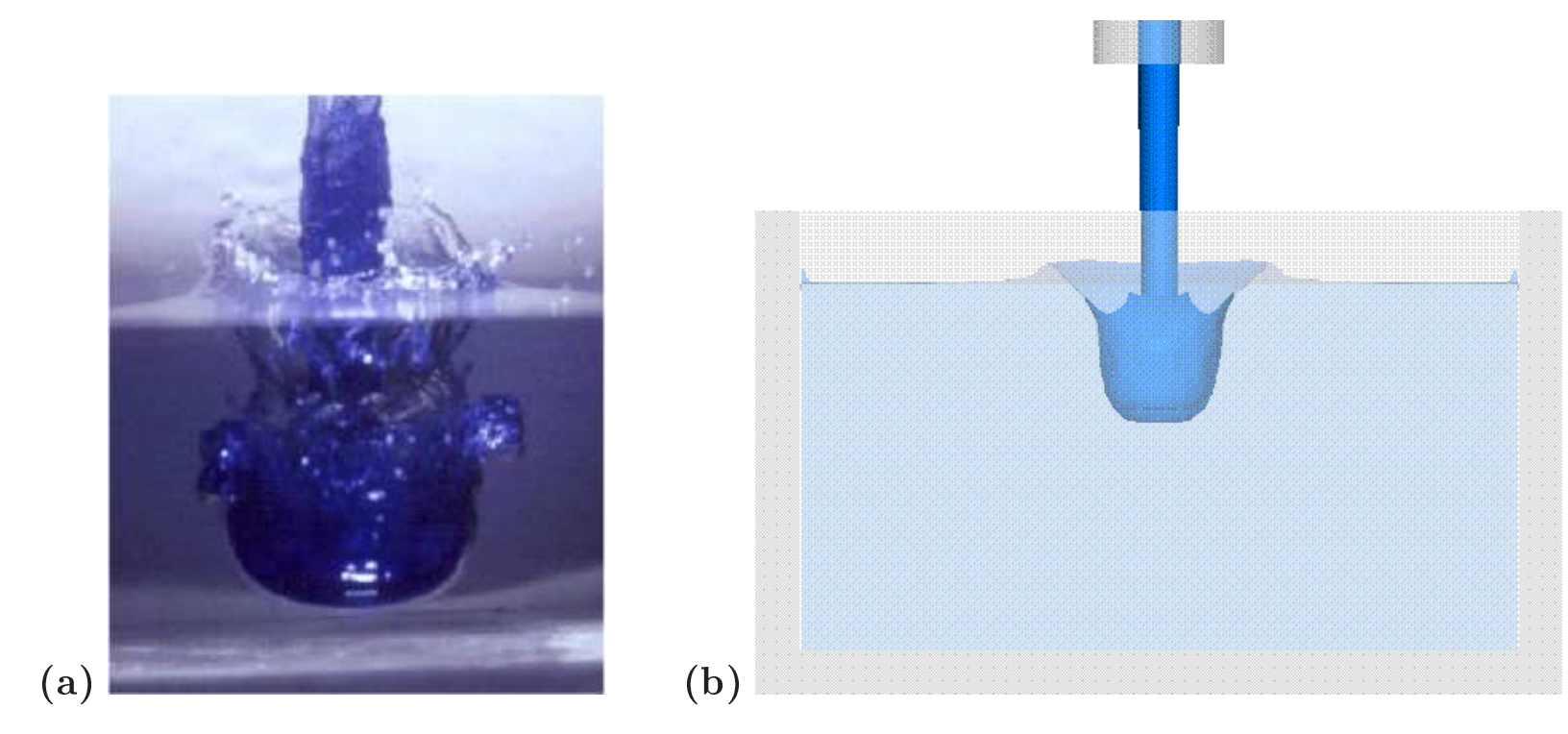

(c)

Figura 5.84: Comparação da simulação numérica obtida pelo código Freeflow 3D equipado com as modelagens ADBQUICKEST e $\kappa-\varepsilon$ e o experimento realizado por Storr e Behnia, para o caso em que $R e=3000$, mostrando: (a) visualização experimental; (b) a visão frontal da simulação numérica; (c) visão 3D da simulação numérica. 


\subsubsection{Colapso de um bloco de fluido com obstáculos}

O problema do colapso de fluido com posterior interação com obstáculos, já apresentado na subseção 5.2.4.4, é retomado e simulado usando o código Freeflow $3 D$ atualizado com o esquema ADBQUICKEST e o modelo $\kappa-\varepsilon$.

Novamente é considerado um tanque de $1.6 \mathrm{~m}$ de comprimento, $0.6 \mathrm{~m}$ de largura e $0.6 \mathrm{~m}$ de altura contendo dois blocos de água em equilíbrio hidrostático, e uma estrutura fixa de $0.12 \mathrm{~m}$ $\times 0.12 \mathrm{~m} \times 0.6 \mathrm{~m}$. O primeiro bloco medindo $0.3 \mathrm{~m}$ de comprimento por $0.6 \mathrm{~m}$ de largura por $0.3 \mathrm{~m}$ de altura e o segundo $1.3 \mathrm{~m}$ de comprimento por $0.6 \mathrm{~m}$ de largura por $0.03 \mathrm{~m}$ de altura. Três configurações são consideradas no estudo, a saber:

- Configuração I: uma estrutura está localizada a $0.5 \mathrm{~m}$ do primeiro bloco de água e 0.24 m da parede lateral (ver Figura 5.85);

- Configuração II: uma estrutura está posicionada $0.5 \mathrm{~m}$ do primeiro bloco de água e junto a parede de fundo (ver Figura 5.86);

- Configuração III: duas estruturas, uma posicionada a $0.5 \mathrm{~m}$ do primeiro bloco de água e $0.10 \mathrm{~m}$ da parede frontal, e a outra estrutura localizada a $0.5 \mathrm{~m}$ do primeiro bloco e junto à parede ao fundo, como mostra a Figura 5.87.

Para a simulação do problema fluido-estrutura nas três configurações anteriores, os seguintes dados foram adotados:

- Dimensão do domínio: $1.6 \mathrm{~m} \times 0.6 \times 0.6 \mathrm{~m}$;

- Malha: $160 \times 60 \times 60(\delta x=\delta y=\delta z=0,010 \mathrm{~m})$;

- Constante gravitacional: $g=9.81 \mathrm{~ms}^{-2}$;

- Coeficiente de viscosidade cinemática: $\nu=1.0 \times 10^{-6} \mathrm{~m}^{2} \mathrm{~s}^{-1}$;

- Parâmetros de escala: $L=0.30 \mathrm{~m}$ e $U=\sqrt{g L}=1.715517 \mathrm{~ms}^{-1}$;

- Número de Reynolds: $R e=U L / \nu=514.655,22$;

- Número de Froude: $F r=U / \sqrt{g L}=1.0$.

O critério de convergência na equação de Poisson foi de $1.0 \times 10^{-8}$ e o tamanho do passo temporal de $5.95 \times 10^{-5}$. O número de Reynolds crítico $y_{c}^{+}$e as constantes $K$ e $B$ nas leis de parede assumiram os seguintes valores: $y_{c}^{+} \approx 11.068, K=0.41$ e $B=5.0$, respectivamente. No tempo $t=0 \mathrm{~s}$, os blocos de fluido são postos sob à ação da gravidade e ambos sofrem colapso. O movimento resultante do volume da água após o colapso em diferentes tempos estão mostrados nas Figuras 5.88, 5.89 e 5.90 para as configurações Configuração I, Configuração II e Configuração III, respectivamente. 
Pode-se dizer por essas figuras que o método numérico simulou bem a interação da superfície livre com as estruturas e que essas simulações são bastantes consistentes com àquelas apresentadas por Raad e Bidoae [109].

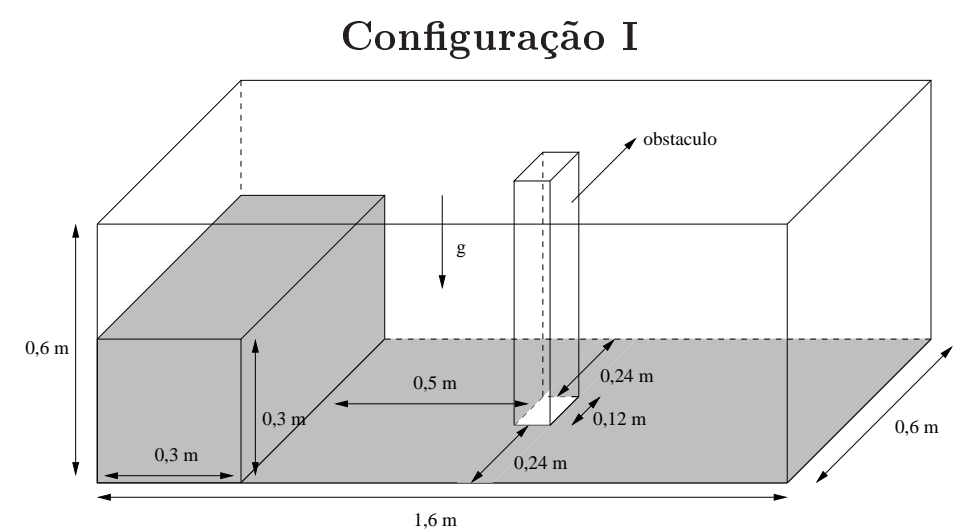

Figura 5.85: Geometria do problema do colapso de fluido com obstáculo localizado no centro.

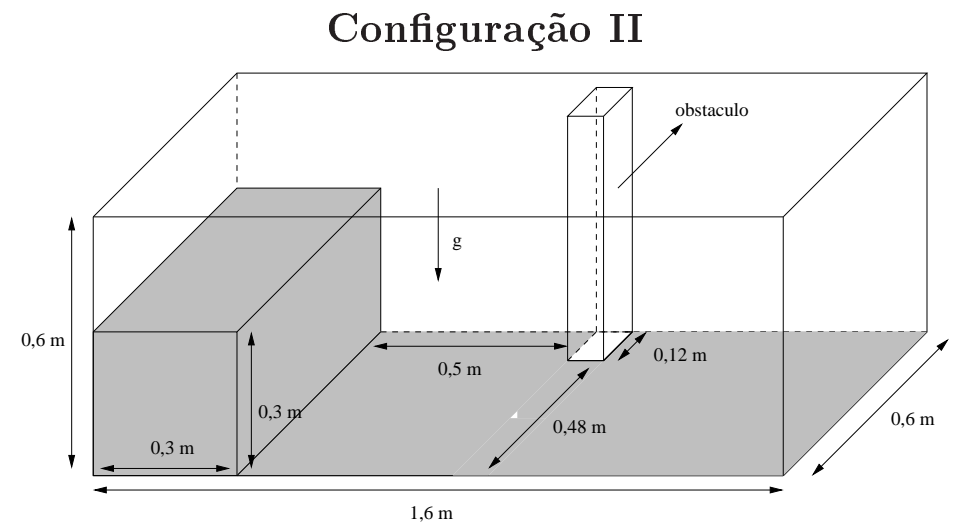

Figura 5.86: Geometria do problema do colapso de fluido com obstáculo localizado junto a parede.

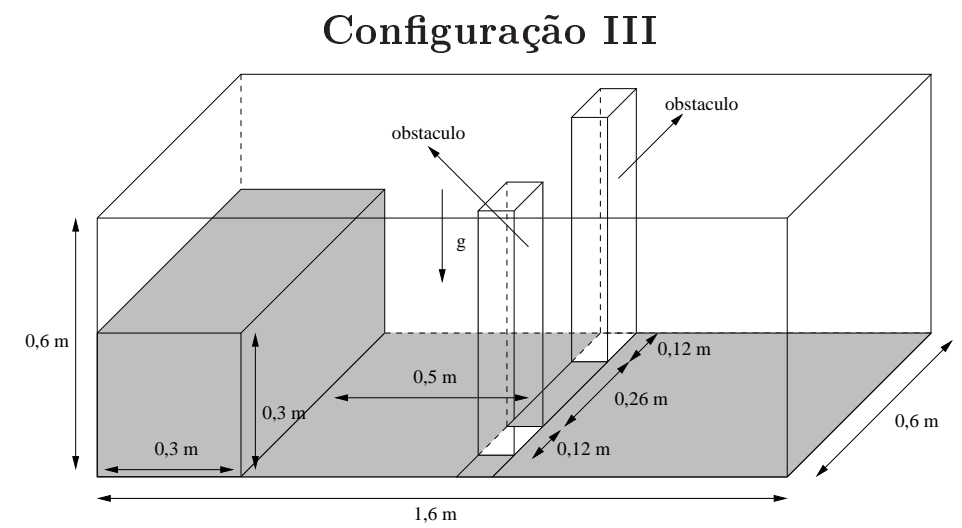

Figura 5.87: Geometria do problema do colapso de fluido com dois obstáculos. 

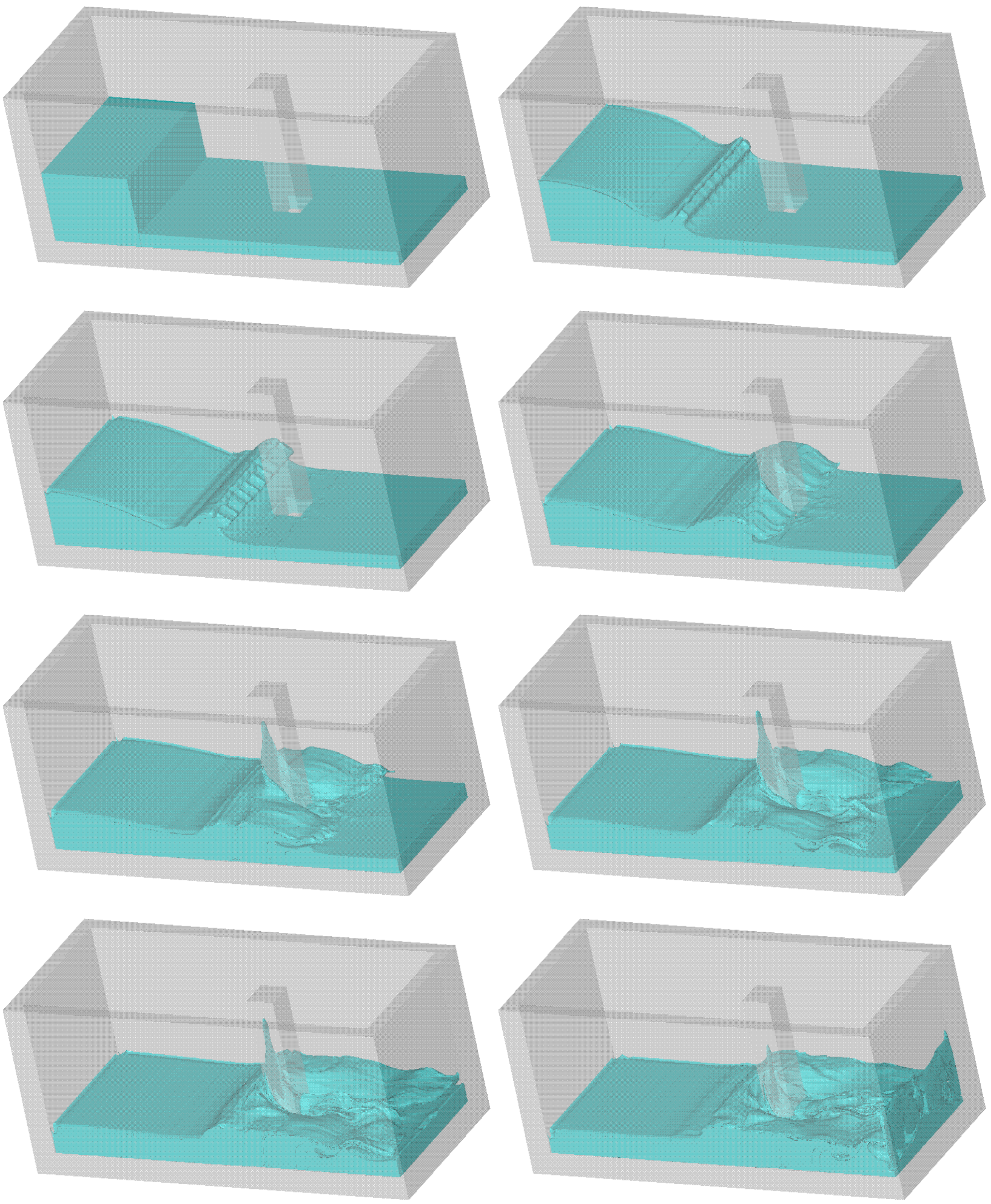

Figura 5.88: Simulação numérica 3D usando o esquema ADBQUICKEST e o modelo $\kappa-\varepsilon$ do problema interação fluido-estrutura com o obstáculo localizado no centro (Configuração I). 

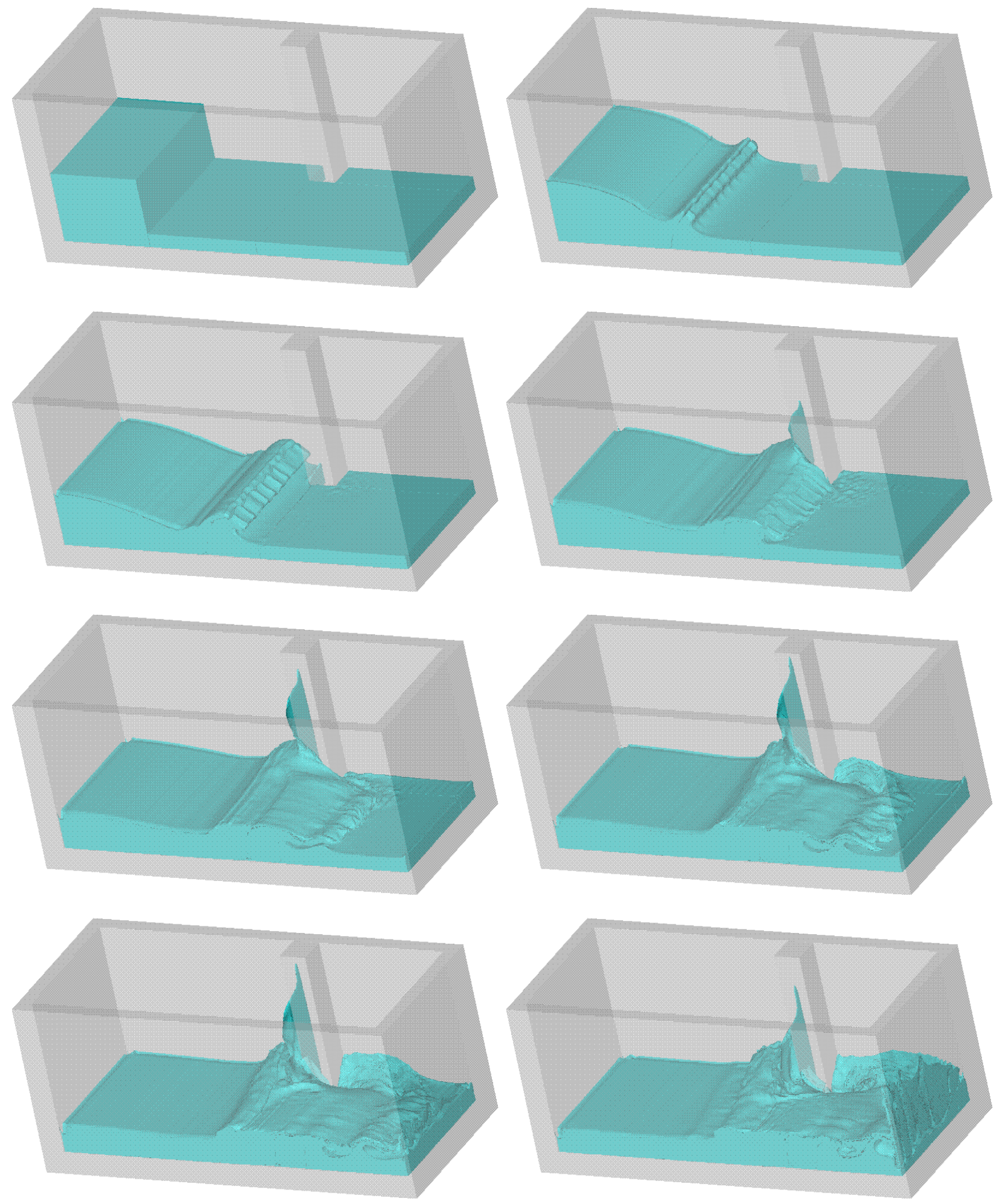

Figura 5.89: Simulação numérica 3D usando o esquema ADBQUICKEST e o modelo $\kappa-\varepsilon$ do problema interação fluido-estrutura com o obstáculo localizado junto a parede (Configuração II). 

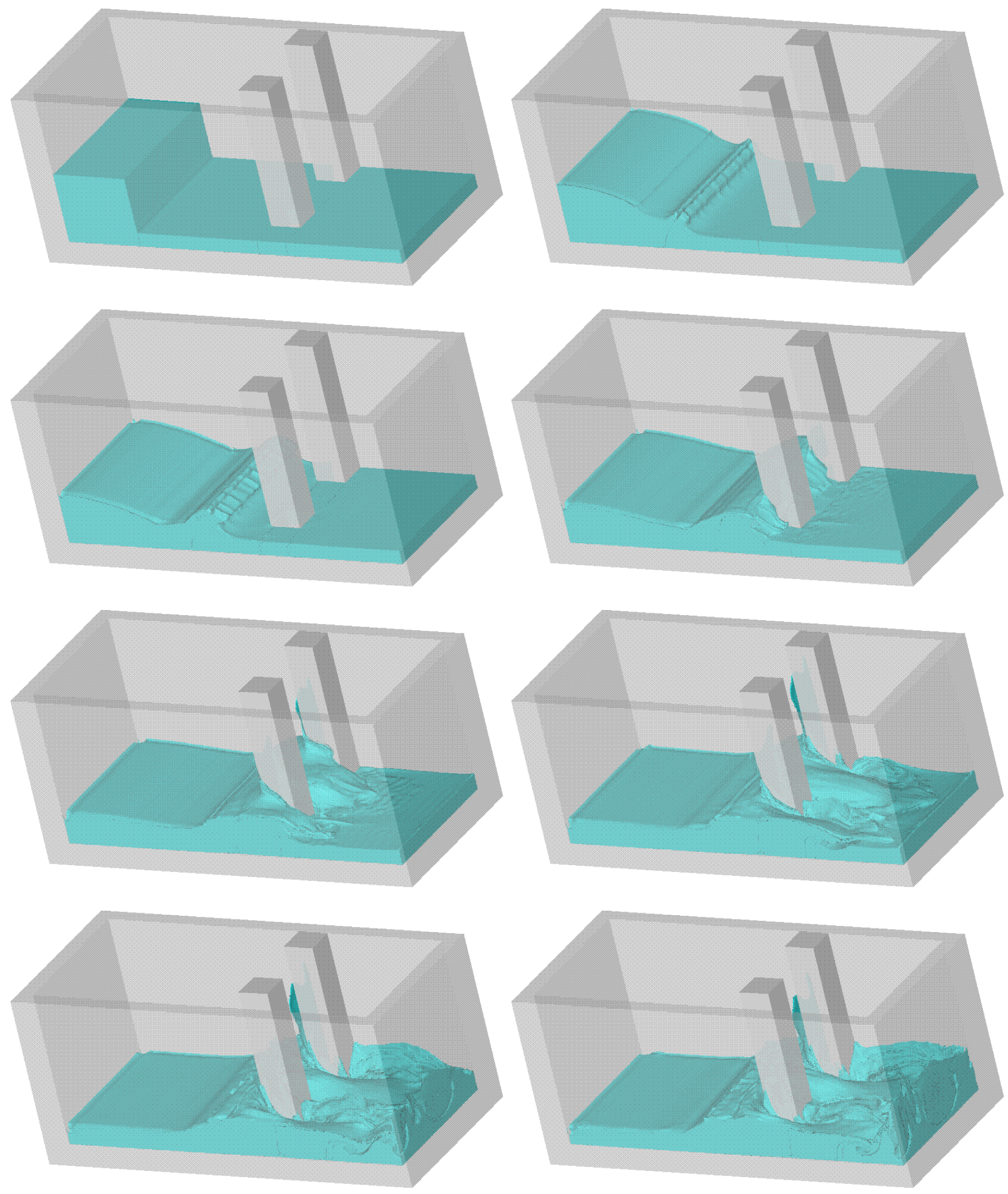

Figura 5.90: Simulação numérica 3D usando o esquema ADBQUICKEST e o modelo $\kappa-\varepsilon$ do problema interação fluido-estrutura com dois obstáculos (Configuração III). 


\subsubsection{Comentários}

Em geral, os códigos Freeflow 2D e 3D adaptados com o modelo $\kappa-\varepsilon$ e esquema de advecção ADBQUICKEST foram aplicados para simular problemas altamente complexos. De acordo com os resultados numéricos, ambos os códigos podem, de fato, simular com sucesso escoamentos turbulentos confinados e com superfície livre..

Os resultados numéricos 2D apresentados aqui estão publicados no periódico Mathematical Problems in Engineering (ver Ferreira et al. [39]). E os resultados numéricos sobre escoamentos 3D turbulentos com superfícies livres estão sendo reunidos em um artigo para ser submetido em um periódico internacional. 


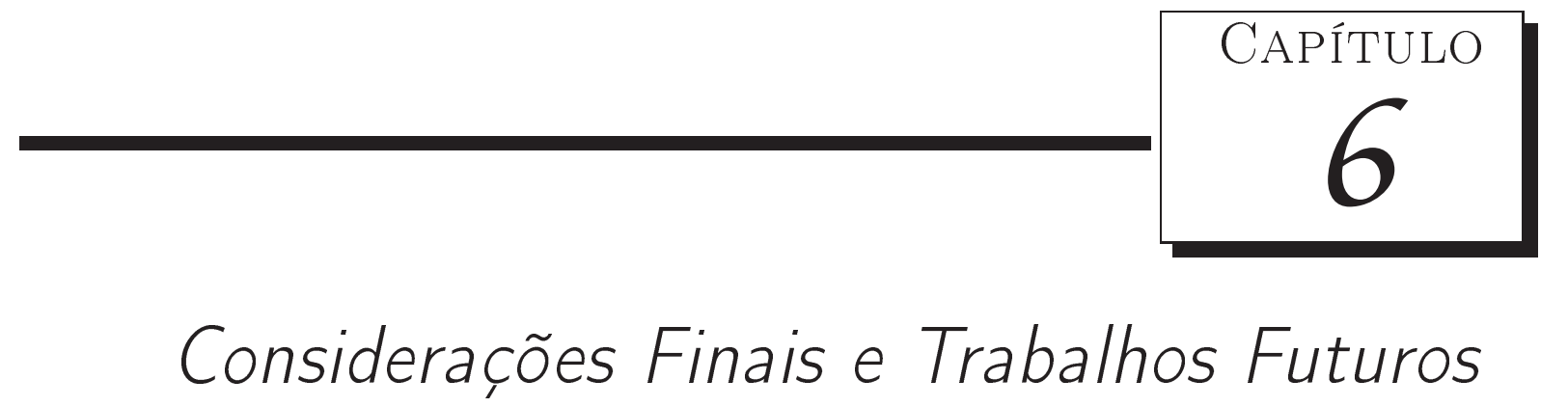

O objetivo deste projeto de doutorado foi analisar e implementar um novo esquema "upwind" de alta resolução que satisfaz os critérios de estabilidade CBC e TVD, para a discretização dos termos advectivos (lineares e não lineares ) de equações de conservações gerais e das equações de Navier-Stokes. Outro objetivo foi incorporar a associação das modelagens ADBQUICKEST e $\kappa-\varepsilon$ no ambiente de simulação Freeflow $2 D$ e $3 D$ para a simulação computacional de problemas de escoamentos incompressíveis com superfícies livres móveis.

Antes de aplicar o esquema ADBQUICKEST em problemas 2D e 3D, vários problemas transientes 1D foram resolvidos com uma variedade de condições iniciais (descontínuas ou não), tais como, transporte de escalares, problemas de Riemann para as equações de Burgers, Euler e águas rasas. O propósito foi mostrar a flexibilidade e a robustez desse esquema em problemas 1D. Os resultados numéricos obtidos nesses problemas foram comparados com soluções analíticas e dados numéricos. De um modo geral, pode-se dizer que o esquema ADBQUICKEST mostrou-se efetivo em simular essa classe de problemas.

Com o sucesso obtido na simulação de problemas 1D, o esquema ADBQUICKEST foi, então, aplicado para a solução de problemas 2D e 3D newtonianos de caráter predominantemente convectivo e em regime laminar. Em particular, seu desempenho foi examinado no problema da expansão brusca, de um jato livre incidindo sobre uma superfície rígida e colapso de fluido. Os resultados numéricos obtidos foram comparados com dados experimentais, soluções analíticas e dados numéricos de outros autores. No caso do problema da expansão brusca, os resultados numéricos obtidos utilizando o esquema ADBQUICKEST mostrou estar em boa concordância com os dados experimentais/numéricos de Armaly et al. [6] e com os dados numéricos de Stuart e Dochan [130]. Com respeito a simulação de um jato livre, os resultados numéricos foram comparados com a solução analítica de Watson [155], e pode-se observar boa concordância. 
Sobre a simulação do colapso de fluido, os resultados numéricos foram confrontados com os dados experimentais de Martin e Moyce [93] e de Koshizuka e Oka [70]. Observou-se neste caso boa concordância com dados experimentais. E ainda, foram apresentadas quatro simulações numéricas 3D de problemas envolvendo superfícies livres a vários valores do número de Reynolds, como por exemplo, um ressalto hidráulico com transição para turbulência, o experimento de Taylor [135], um jato livre oscilante e o colapso de um bloco de fluido com obstáculo. O objetivo aqui foi mostrar que os códigos Freeflow $2 D$ e $3 D$ equipados com a modelagem ADBQUICKEST são ferramentas úteis para simular numericamente escoamentos incompressíveis altamente complexos.

O esquema ADBQUICKEST foi implementado também no código Freeflow $2 D$ equipado com o modelo $\kappa-\varepsilon$ para a simulação de problemas $2 \mathrm{D}$ em regime turbulento. Nesta caso foram simulados os problemas da expansão brusca, e os resultados mostraram boa concordância com os dados numéricos de Thangam e Speziale [138] e os dados experimentais de Eaton e Johnston [33]. Para o jato livre, os resultados numéricos foram comparados com as solução analítica de Watson [155], cujos resultados mostraram ser satisfatórios. Além disso, foram simulados um jato planar fluindo numa porção de fluido em repouso e o problema "sluice gate", na qual, os resultados numéricos apresentaram várias estruturas turbilhonares por todo o domínio de cálculo.

A combinação do esquema ADBQUICKEST com a modelagem $\kappa-\varepsilon$ da turbulência foi, então, utilizada para a simulação de escoamentos 3D com superfícies livres em regime turbulento. Nesse caso, para validação e verificação da modelagem numérica, foram considerados os seguintes problemas: jato livre 3D incidindo sobre uma superfície rígida impermeável e o colapso de um bloco de fluido. Para o jato 3D, os resultados numéricos foram comparados com a solução analítica de Watson, apresentando boa concordância. No colapso, os resultados numéricos foram comparados com os dados experimentais de Martin e Moyce [93] e Koshizuka e Oka [70], e os resultados foram bastante satisfatórios.

Com base nos resultados numéricos apresentados nesta tese, fica claro que é extremamente benéfico estimar os termos não-lineares advectivos das equações de transporte pelo esquema "upwind" limitado de alta resolução ADBQUICKEST. As propriedades dissipativas dessa aproximação são suficientes para controlar as possíveis oscilações/instabilidades produzidas durante os cálculos. Assim, o esquema de advecção aqui apresentado e o modelo de turbulência investigado contribuíram para a área de simulação numérica de escoamentos 3D transientes com superfícies livres móveis.

A pesquisa científica descrita neste trabalho pode ser incrementada de várias maneiras, sobretudo no que diz respeito à combinação de esquemas de diferenças "upwind" de alta resolução e modelagem da turbulência por duas equações. Os aspectos mais relevantes dos temas que serão considerados na pesquisa futura incluem:

- a combinação do esquema "upwind" ADBQUICKEST e a modelagem $\kappa-\varepsilon$ da turbulência 
podem ser aplicado também numa variedades de problemas de escoamentos incompressíveis com superfícies livres móveis. Como exemplos pode-se citar os problemas envolvendo tensão superficial, transferência de calor, fenômenos de convecção e difusão que ocorrem nos fluidos não-newtonianos com efeitos de elasticidade e os problemas multifásicos (em particular gás-sólido).

- o problema fundamental da precisão do esquema ADBQUICKEST em escoamentos 3D realísticos é um assunto importante que precisa ser bem explorado. Para tanto, a simulação das diferentes estruturas poligonais do ressalto hidráulico de Ellegard e colaboradores (ver Ellegaard et al. [36] e Ellegaard et al. [35]) constitui um excelente exemplo de validação. Além disso, objetiva-se comparar os resultados das simulações do problema interação fluido-estrutura com os dados experimentais de Raad e Bidoae [109].

- os códigos Freeflow atuais empregam malhas uniformes para discretizar o domínio de solução. Um refinamento localizado da malha computacional é, então, fator essencial para uma análise mais elaborada dos métodos aqui apresentados, quando aplicados nas vizinhanças de paredes rígidas e camadas cizalhantes, pois é muito importante resolver com boa precisão a camada-limite, uma vez que é nela onde os gradientes elevados aparecem.

- a captura correta do choque, isto é a ausência de pontos soluções no choque, é um tema atual e merece ser investigado no contexto da pesquisa aqui apresentada. Para tanto, pretende-se associar os esquemas ADBQUICKEST com a técnica RCM (Randon Choice Method) de Glimm [48] (ver também aplicação em [163]). 


\section{Referências Bibliográficas}

[1] K. Alhuimaizi. Flux-limiting solution techniques for simulaion of reaction-diffusion-convection system. Communications in Nonlinear Sciences and Numerical Simulation, 12:953-965, 2007.

[2] M.A. Alves, P.J. Oliveira, and F.T. Pinho. A convergent and universally bounded interpolation scheme for the treatment of advection. International Journal for Numerical Methods in Fluids, 41:47-75, 2003.

[3] W.F. Ames. Nonlinear partial differential equations in engineering, Vol.I. Academic Press, New York, 1965.

[4] A.A. Amsden and F.H. Harlow. A simplified mac technique for incompressible fluid flow calculations. Journal of Computational Physics, 6:332-335, 1970.

[5] D.A. Anderson, J.C. Tannehill, and R.H. Pletcher. Computational fluid mechanics and heat transfer. Hemisphere Publishing Corporation, New York, 1984.

[6] B.F. Armaly, F. Durst, J.C.F. Pereira, and B. Schonung. Experimental and theoretical investigation of backward-facing step flow. Journal of Fluid Mechanics, 127:473-496, 1983.

[7] M. Arora and P.L. Roe. A well-behaved tvd limiter for high-resolution calculations of unsteady flow. Journal of Computational Physics, 132:3, 1997.

[8] B. Ataie-Ashtiani and L. Farhadi. A stable moving-particle semi-implicit method for free surface flows. Fluid Dynamics Research, 38:241-256, 2006.

[9] C.T. Avedisian and Z. Zhao. The circular hydraulic jump in low gravity. In Proceedings of the Royal Society of London, Series A, volume 456, pages 2127-2151, 2000. 
[10] D.S Balsara and C.-W. Shu. Monotonicity preserving weighted essentially non-oscillatory schemes with increasingly high order of accuracy. Journal of Computational Physics, 160:405-452, 2000.

[11] G.K. Batchelor. An introduction to fluid dynamics. Cambridge University Press, 1967.

[12] G. Billet and O. Louedin. Adaptive limiters for improving the accuracy of the muscl approach for unsteady flows. Journal of Computational Physics, 170:161-183, 2001.

[13] J. Boussinesq. Memoires presentes par divers savants sciences mathematique at physiques theorie de l'ecoulement tourbillant. Academie des Sciences, Paris, 23, 1877.

[14] P. Bradshaw. Turbulence, Topics in Applied Physics V. 12. Springer-Verlag, Berlin, 1976.

[15] J. Burguete, P. García-Navarro, and J. Murillo. Preserving bounded and conservative solutions of transport in one-dimensional shallow-water flow with upwind numerical schemes: Application to fertigation and solute transport in rivers. International Journal for Numerical Methods in Fluids, 456:1731-1764, 2008.

[16] J.W.M. Bush and J.M. Aristoff. The influence of surface tension on the circular hydraulic jump, journal of fluid mechanics. Journal of Fluid Mechanics, 489:229-238, 2003.

[17] J.W.M. Bush, J.M. Aristoff, and A.E. Hosoi. An experimental investigation of the stability of the circular hydraulic jump. Journal of Fluid Mechanics, 558:33-52, 2006.

[18] A.F. Castelo, N. Mangiavacchi, M.F. Tomé, J.A. Cuminato, V.G. Ferreira, and S. McKee. Surface tension implementation for gensmac2d. Journal of the Brazilian Society of Mechanical Sciences, 4:523-532, 2001.

[19] A.F. Castelo, M.F. Tomé, C.N.L. César, S. McKee, and J.A. Cuminato. reeflow: An integrated simulation system for three-dimensional free-surface flows. Journal of Computers and Visualization in Science, 2:199-210, 2000.

[20] S.K. Choi, H.Y. Nam, and M. Cho. A comparison of high-order bounded convection schemes. Computational Methods in Applied Mechanics and Engineering, 121:281-301, 1995.

[21] A.J. Chorin. Numerical solution of the navier-stokes. Mathematics of Computation, 2:745-762, 1968.

[22] R. Courant, K. Friedrichs, and H. Lewy. On the partial differential equations of mathematical physics. IBM Journal Research and Development, 11:215-234, 1967.

[23] R. Courant, E. Isaacson, and M. Rees. On the solution of nonlinear hyperbolic differential equations by finite differences. Community Pure Applied Mathematics, 243-255:5, 1952. 
[24] J.O. Cruickshank and B.R. Munson. Viscous fluid buckling of plane and axisymmetric jets. Journal of Fluid Mechanics, 113:221-239, 1981.

[25] R.A. Dalrymple, O. Knio, D.T. Cox, M. Gesteira, and S. Zou. Using a lagrangian particle method for deck overtoppingem equilibrio hidrostático. ASCE, Reston, Va., pages 1082-1091, 2002.

[26] M.S. Darwish. A new high-resolution scheme based on the normalized variable formulation. Numerical Heat Transfer, 353-373:24, 1993.

[27] S.F. Darwish. Numerical methods for partial differential equations. Shock capturing, p. 175-198, 1986.

[28] F.M. Denaro. On the applications of the helmoltz-hodge decomposition in projection methods for incompressible flows with general boundary conditions. International Journal for Numerical Method in Fluid, 43:43-69, 2003.

[29] C.R. Dennis, D.B. Ingham, and R.N. Cook. Finite-difference methods for calculating flows in three dimensions. Journal of Computational Physics, 33:329-335, 1979.

[30] M.O. Deville. Numerical experiments on the mac code for a slow flow. Journal of Computational Physics, 15:362-374, 1974.

[31] T. Dubois, F. Jauberteau, and R. Teman. Dynamic multilevel methods and the numerical simulation of turbulence. University Press, Cambridge, 1999.

[32] P.A. Durbin. On the $\kappa-\varepsilon$ stagnation point anomaly. Journal Heat and Fluid Flow, Cambridge, 17:89-90, 1996.

[33] J. Eaton and J.P. Johnston. Turbulent flow reattachment: an experimental study of the flow and structure behind a backward-facing step. Stanford University, TR MD-39, CA, 1980 .

[34] D. Elkaim, M. Reggio, and R. Camarero. Simulating two-dimensional turbulent flow by using the $\kappa-\varepsilon$ model and the vorticity-stream function formulation. International Journal for Numerical Methods in Fluids, 14:961-980, 1992.

[35] C. Ellegaard, A.E. Hansen, A. Haaning, K. Hansen, A. Marcussen, and T. Bohr. Cover illustration: Poligonal hydraulic jumps. Nonlinearity, 12:1-7, 1999.

[36] C. Ellegaard, A.E. Hansen, A. Haaning, K. Hansen, A. Marcussen, T. Bohr, J.L. Hansen, and S. Watanabe. Creating corners in kitchen sinks. Nature, 23:767-768, 1998.

[37] I. Fatkullin and J.S. Hesthaven. Adaptive high-order finite-difference metho for nonlinear wave problems. Journal of Scientific Computing, 16 (1):47-67, 2001. 
[38] V.G. Ferreira. Análise e implementação de esquemas de convecção e modelos de turbulência para simulação de escoamentos incompressiveis envolvendo superfícies livres. Tese de Doutorado - Instituto de Ciências Matemáticas e de Computação (ICMC-USP), São Carlos, SP, 2001.

[39] V.G. Ferreira, A.C. Brandi, F.A. Kurokawa, P. Seleghim Jr., A. Castelo, and J.A. Cuminato. Incompressible turbulent flow simulation using the $\kappa-\varepsilon$ model and upwind schemes. Mathematical Problems in Engineering, 2007, Article ID 12741:26 pages, 2007.

[40] V.G. Ferreira, F.A. Kurokawa, C.M. Oishi, M.K. Kaibara, A. Castelo, and J.A. Cuminato. Evaluation of a bounded high order upwind scheme for $3 \mathrm{~d}$ incompressible free surface flow computations. Mathematics and Computers in Simulation, 79:1895-1914, 2009.

[41] V.G. Ferreira, F.A. Kurokawa, R.A.B. Queiroz, M.K. Kaibara, C.M. Oishi, J.A. Cuminato, A. Castelo, M. F. Tomé, and S. McKee. Assessment of a high-order finite difference upwind scheme for the simulation of convection?diffusion problems. International Journal for Numerical Methods in Fluids, In press, 2008.

[42] V.G. Ferreira, N. Mangiavacchi, M.F. Tomé, A. Castelo, J.A. Cuminato, and S. McKee. Numerical simulation of turbulent free surface flow using two-equation $\kappa-\varepsilon$ viscosity models. International Journal for Numerical Methods in Fluids, 44:347-375, 2004.

[43] V.G. Ferreira, C.M. Oishi, F.A. Kurokawa, M.K. Kaibara, J.A. Cuminato, A. Castelo, N. Mangiavacchi, M.F. Tomé, and S. McKee. A combination of implicit and adaptative upwind tools for the numerical solution of incompressible free surface flows. Communications in Numerical Methods in Engineering, 23:419-445, 2007.

[44] V.G. Ferreira, M.F. Tomé, N. Mangiavacchi, A. Castelo, J.A. Cuminato, A.O. Fortuna, and S. McKee. High-order upwinding and the hydraulic jump. International Journal for Numerical Methods in Fluids, 39:549-583, 2002.

[45] J.A. Ferziger and M. Perić. Computational methods for fluid dynamics. Springer, Germany, 1984.

[46] E. Fontaine, M. Landrini, and M. Tulin. Breaking: Splashing and ploughing phases. In Proceedings of International Workshop on Waterwaves and Floating Bodies, pages 34-38, 2000.

[47] P.H. Gaskell and A.K. Lau. Curvature-compensated convective transport: Smart, a new boudedness-preserving transport algorithm. International Journal for Numerical Methods in Fluids, 8:617-641, 1988. 
[48] J. Glimm. Solution in the large for nonlinear hyperbolic systems of equations. Solution in the large for nonlinear hyperbolic systems of equations, 18:697-715, 1965.

[49] D.M. Greaves. Simulation of viscous water column collapse using adapting hierarchical grids. International Journal for Numerical Methods in Fluids, 50:693-711, 2006.

[50] E.S. Gross, J.R. Koseff, and S.G. Monismith. Evaluation of advective scheme for estuarine salinity simulation. Journal of Hydraulic Engineering, 125:32-46, 1999.

[51] M. Gómez-Gesteira and R.A. Dalrymple. Using a three-dimensional smoothed particle hydrodynamics method for wave impact on a tall structure. J. Waterway, Port Coast. Ocean Eng., 130:63-69, 2004.

[52] F.H. Harlow and P.I. Nakayama. Turbulence transport equations. The Physics of Fluids, 10:2323-2333, 1967.

[53] F.H. Harlow and E. Welch. Numerical calculation of time-dependent viscous incompressible flow of fluid with free surface. The Physics of Fluids, 8:2182-2189, 1965.

[54] A. Harten. High resolution schemes for hyperbolic conservation laws. Journal of Computational Physics, 49:357-393, 1983.

[55] A. Harten. On a class of high resolution total-variation-stable finite-difference schemes. SIAM Journal of Numerical Analysis, 21:1-23, 1984.

[56] A. Harten. Eno scheme with subcell resolution. Journal of Computational Physics, 83:148-184, 1989.

[57] A. Harten, B. Engquist, S. Osher, and S. Chakravarthy. Unifomly high order essentially nonoscillatory schemes, iii. Journal of Computational Physics, 71:321-303, 1987.

[58] M. Herrmann, G. Blanquart, and V. Raman. Flux corrected finite volume scheme for preserving scalar boundedness in reacting large-eddy simulations. AIAA Journal, 44:2879-2886, 2006.

[59] C. Hirsch. Numerical computation of internal and external flows, Volumes 1 e 2. John Wiley \& Sons, Chichester, 1988.

[60] C.W. Hirt and J.L. Cook. Free surface stress conditions for incompressible flow calculations. Journal of Computational Physics, 2:403-411, 1972.

[61] C.W. Hirt and J.P. Shannon. Calculating three dimensional flows around structures and over rough terrain. Journal of Computational Physics, 10:324-340, 1968. 
[62] W.V.D. Hodge. The Theory and Applications of Harmonic Integrals. Cambridge University Press, Cambridge, 1952.

[63] H.E. Huppert. The intrusion of fluid mechanics into geolog. Journal of Fluid Mechanics, 173:557-594, 1986.

[64] M.Y. Hussaini, V. van Leer, and J. van Rosendale. Upwind and High-Resolution Scheme. Springer-Verlag, Heidelberg, 1997.

[65] W.P. Jones and B.E. Launder. The prediction of laminarization with a two-equation model of turbulence. International Journal Heat Mass Transfer, 15:301?314, 1972.

[66] M.K. Kaibara, V.G. Ferreira, and H.A. Navarro. Upwinding finite-difference schemes for convection dominated problems, Part I: Theoretical Results. Notas do ICMC-USP, 80, 2004 .

[67] V.I. Karpman. Non-linear waves in dispersive media. Fluid Mechanics, Pergamon, Elmsford: New York, 1975.

[68] M. Kato and B.E. Launder. The modelling of turbulent flow around stationary and vibrating square cylinders. In Proceedings 9th. Symposium on Turbulent Shear Flows, pages 10-14, 1993.

[69] J. Kim and P. Moin. Application of a fractional-step methods to incompressible navier-stokes equations. Journal of Computational Physics, 59:308-323, 1985.

[70] S. Koshizuka and S. Oka. Moving-particle semi-implicit method for fragmentation of incompressible fluids. Nuclear Science and Engineering, 123:421-434, 1996.

[71] R. Kupferman and E. Tadmor. A fast high-resolution second-order central scheme for incompressible flows. Proc. Nat. Acad. Sci., 94:4848-4852, 1997.

[72] M. Kurihara. A consideration about hydraulic jump. Rep. Res. Inst. Fluid Eng., Kyusyu Imp. Univ., 3:11, 1946.

[73] O.A. Ladyzhenskaja. The mathematical theory of viscous incompressible flow. Gordon and Breach, New York, 1963.

[74] B.E. Launder and D.B.Spalding. The numerical computation of turbulent flows. International Journal for Numerical Methods in Fluids, 15:127-146, 1974.

[75] B.E. Launder and D.B. Spalding. Lectures in mathematical models of turbulence. London: Academic Press, 1972. 
[76] P.D. Lax. Weak solutions of nonlinear hyperbolic equations and their numerical computation. Communication in Pure Applied Mathematics, 7:59-193, 1954.

[77] P.D. Lax and B. Wendroff. Systems of conservations laws. Communications in Pure and Applied Mathematics, 13:217-237, 1960.

[78] H. Lei, L-Z. Wang, , and Z-N. Wu. Applications of upwind and downwind schemes for calculating electrical conditions in a wire-plate electrostatic precipitator. Journal of Computational Physiscs, 193:697-707, 2004.

[79] B.P. Leonard. The quick algorithm: a uniformly third-order finite difference method for highly convective flows. Comput. Methods Appl. Mech. Eng, 71:321-303, 1979.

[80] B.P. Leonard. Simple high-accuracy resolution program for convective modeling of discontinuities. International Journal for Numerical Methods in Fluids, 8:1291-1318, 1988.

[81] B.P. Leonard. The ultimate conservative difference scheme applied to unsteady one-dimensional advection. Computer Methods in Applied Mechanics and Engineering, 88:17-74, 1991.

[82] R.J. LeVeque. Finite Volume Methods for Hyperbolic Problems. Cambridge University Press, New York, 2004.

[83] R.J. LeVeque. Finite difference methods for ordinary and partial differential equations, steady-state and time-dependent problems. In SIAM Society for Industrial and Applied Mathematics, Philadelphia,, 2007.

[84] D. Levy, G. Puppo, and G. Russo. A fourth-order central weno scheme for multidimensional hyperbolic systems of conservation laws. SIAM Journal of Scientific Computing, 24:480-506, 1991.

[85] Y. Li. Wave number-extended high-order upwind-biased schemes for convective scalar transport. Journal of Computational Physiscs, 133:235-255, 1997.

[86] C.-L. Lin, H. Lee, T. Lee, and L.J. Weber. A level set characteristic galerkin finite element method for free surface flows. International Journal for Numerical Methods in Fluids, 49:521-547, 2005.

[87] C.H. Liu and D.Y.C. Leung. Development of a finite element solution for the unsteady navier-stokes equations using projection method and fractional- $\theta$-scheme. Computer Methods in Applied Mechanics and Engineering, 190(3):4301-4317, 2001. 
[88] R. Löhner, C. Yang, and E. Onate. Simulation of flows with violent free surface motion and moving objects using unstructured grids. International Journal for Numerical Methods in Fluids, 53:1315-1338, 2007.

[89] J.M. MacDonough. Introductory lectures on turbulence: physics, mathematics and modeling. Department of Mechanical Engineering and Mathematics, University of Kentucky, 2004 .

[90] M. Maleki, M. Habibi, R. Golestanian, N.M. Ribe, and D. Bonn. Liquid rope coiling on a solid surface. Physical Review Letter, 93:214, 2004.

[91] N. Mangiavacchi, R. Gundlapalli, and R. Alchavan. Dynamics of a turbulent jet interacting with a free surface. ASME Symposium on Free Surface Turbulence, pages Lake Tahoe, NV, USA, 1994.

[92] H. Manouzi and M. Fortin. A treatment of wall boundaries for turbulent flows by the use of a transmission finite element method. International Journal for Numerical Methods in Engineering, 31:113-126, 1991.

[93] J.C. Martin and W.J. Moyce. An experimental study of the collapse of liquid columns on a rigid horizontal plate. Philosophical Transactions of the Royal Society of London, series A. Mathematical, Phisical and Engineering Sciences, 244:312-324, 1952.

[94] W.D. McComb. The physics of fluid turbulence. Oxford University Press, New York, 1990.

[95] S. McKee, M.F. Tomé, V.G. Ferreira, J.A. Cuminato, A. Castelo, F.S. Sousa, and N. Mangiavacchi. The mac method. Computers \& Fluids, 37:907-930, 2008.

[96] G.L. Mellor and H.J. Herring. A survey of the mean turbulent field closure models. AIAA Journal, 11:590-599, 1973.

[97] D. Mouaze, F. Murzyn, and J.R. Chaplin. Free surface length scale estimation in hydraulics jumps. Journal of Fluids Engineering, 127:1191-1193, 2005.

[98] L.R. Mudryk and N.W. Murray. A fast resolution flux-conservative algorithm designed for planet-disk interactions. New Astronomy, In Press, 2008.

[99] B.D. Nichols and C.W. Hirt. Methods for calculating multidimensional, transient free surface flows past bodies. In Proceedings of the First International Conf. On Num. Ship Hydrodynamics, 1975.

[100] H.L. Norris and W.C. Reynolds. Turbulent channel flow with a moving wavy boundary. Stanford University Departament of Mechanics Engineering, pages TR TF-7, 1975. 
[101] M. Nóbrega, O.S. Carneiro, F.T. Pinho, G.S. Paulo, M.F. Tomé, A. Castelo, and J.A. Cuminato. The phenomenon of jet buckling: experimental results and numerical predictions. Proceedings of The Polymer Processing Society 23rd Annual Meeting, Salvador/Brasil, 2007.

[102] C.M. Oishi. Análise e implementação de métodos implicitos e de projeção para escoamentos com superfície livre. In Tese de Doutorado - Instituto de Ciências Matemáticas e de Computação (ICMC-USP), volume São Carlos, 2008.

[103] C.M. Oishi, M.F. Tomé, J.A. Cuminato, and S. McKee. An implicit technique for solving 3d low reynolds number moving free surface flows. Journal of Computational Physics, page DOI:10.1016/j.jcp.2008.04.017, 2008.

[104] C.M. Oldenburg and K. Pruess. Simulation of propagating fronts in geothermal reservoirs with the implicit leonard total variation diminishing scheme. Geothermics, 29:1-25, 2000.

[105] A. Ouahsine and H. Smaoui. Flux-limiter schemes for oceanic tracers: application to the english channel tidal model. Computer Methods in Applied Mechanics and Engineering, 1999:307-325, 179.

[106] S.V. Patankar. Numerical heat transfer and fluid flows. Hemisphere Publishing Co., New York, 1980.

[107] V.C. Patel. Perspective: flow at high reynolds number and over rough surfaces - achilles heel of cfd. Transaction of the ASME Journal on Fluids Engineering, 120:434-444, 1998.

[108] B. Perot and R. Nallapati. A moving unstructured staggered mesh method for the simulation of incompressible free-surface flows. Journal of Computational Physics, 184:192-214, 2003.

[109] P.E. Raad and R. Bidoae. The three-dimensional eulerian-lagrangian marker and micro cell method for the simulation of free surfaces flows. Journal of Computational Physics, 203:668-699, 2004.

[110] L. Rayleigh. On the theory of long waves and bores. In Proceedings of the Royal Society of London, Series A, volume 90, pages 324-328, 1948.

[111] O. Reynolds. On the dynamical theory of incompresssible viscous fluids and the determination of the criterion. Phil. Trans. Roy. Soc. London Ser. A, 186:123-164, 1895.

[112] N.M. Ribe, H.E. Huppert, M.A. Hallworth, M. Habibie, and D. Bonn. Multiple coexisting states of liquid rope coiling. Journal of Fluid Mechanics, 555:275-297, 2006. 
[113] D.i Robinson and H.A. Hassan. Two-equation turbulence closure model for wall bounded and free shear flows. AIAA Journal, 115:147-152, 1994.

[114] W. Rodi. Turbulence models and their application in hydraulics - A state of the Art Review. 2 ed. International Association for Hydraulic Research, University of Karlsruhe, Germany, 1984.

[115] A. Rogerson and E. Melberg. A numerical study of the convergence properties of eno schemes. Journal of Scientific Computing, 18:555, 1994.

[116] S. Le Roy, G. Teyssendre, and G. Laurent. Numerical methods in the simulation of charge transport in solid dielectrics. IEEE Transaction on Dielectrics and Electrical Instalation, 13:239-246, 2006.

[117] S. Sankaranarayanan and H. Suresh Rao. Finite element analysis of free surface flow through gates. International Journal for Numerical Methods in Fluids, 22:375-392, 1996.

[118] L. Shen and D.K.P. Yue. Large-eddy simulation of free-surface turbulence. Journal of Fluid Mechanics, 440:75-116, 2001.

[119] T.W.H. Sheu, C.C. Fang, S.F. Tsai, and C-Y. Huang. On an adaptive monotonic convection-diffesion flux discretization scheme. Computer Methods in Applied Mechanics and Engineering, 173:201-215, 1999.

[120] C.-W. Shu and S. Osher. Efficient implementation of essentially non-oscillatory shock capturing schemes, ii. Journal of Computational Physics, 83:32, 1989.

[121] S.Z. Shuja and B.S. Yilbas. Gas-assisted laser repetitive pulsed heating of a steel surface. In Proceedings Instn. Mech. Engrs. Part C, volume 212, pages 741-757, 1998.

[122] J.M. Silva. Simulação numérica de escoamentos com superfícies livres e com influência da temperatura. In Dissertação de mestrado - Instituto de Ciências Matemáticas e de Computação (ICMC-USP), volume São Carlos, 2002.

[123] L.M. Smith and W.C. Reynolds. On the yakhot-orszag renormalization group method for deriving turbulence statistics and models. Physics of Fluids A, 4:364?390, 1992.

[124] G. Sod. A survey of several finite difference methods for systems of nonlinear hyperbolic conservation laws. Journal of Computational Physics, 27:1, 1978.

[125] D.L. Sondak and R.H. Pletcher. Application of wall functions to generalized nonorthogonal curvilinear coordinate systems. AIAA Journal, 33:33-41, 1995.

[126] B. Song, G.R. Liu, K.Y. Lam, and R.S. Amano. On a higher-order bounded discretization scheme. International Journal for Numerical Methods in Fluids, 32:881-897, 2000. 
[127] D.B Spalding. A novel finite difference formulation for differential expressions involving both first and second derivatives. International Journal for Numerical Methods in Fluids, 4:551-559, 1972.

[128] C.G. Speziale. On non-linear $\kappa-l$ and $\kappa-\varepsilon$ models of turbulence. AIAA Journal, 178:459-475, 1987.

[129] G.J. Storr and M. Behnia. Comparisons between experiment and numerical simulation using a free surface technique of free-falling liquid jets. Exp. Th. Fluids Science, 22:79-91, 1999.

[130] E.R. Stuart and K. Dochan. An upwind differencing scheme for incompressible navier-stokes equations. Applied Numerical Mathematics, 8:43-64, 1991.

[131] P.K. Sweby. High resolution schemes using flux limiters for hyperbolic conservation laws. SIAM Journal of Numerical Analysis, 21(5):995-1011, 1984.

[132] I. Tani. Flow separation in thin liquid layers. Journal of the Physical Society of Japan, $4: 212-215,1948$.

[133] J.C. Tannehill, D.A. Anderson, and R.H. Pletcher. Computational fluid mechanics and heat transfer. 2. ed. Washington, DC: Taylor \& Francis, 1997.

[134] G.I. Taylor. Instability of jets, threads and sheets of viscous fluids. Proc. Int. Cong. Appl. Mech., Springer, Berlin, 1968.

[135] G.I. Taylor. Low-reynolds number flows. National Committee for Fluid Mechanics Films. Illustrated experiments in fluid mechanics, 2o. Ed.:47-54, 1974.

[136] T. Temam. Sur l'approximation de la solution des equations de navier-stokes par la methode de pas fractionnaires (ii). Archieves of Rational Mechanics and Analysis, 33:377-385, 1969.

[137] H. Tennekes and L. Lumley. A first course in turbulence. Masschussets Institute of Technology, USA, 1972.

[138] S. Thangam and C.G. Speziale. Turbulent flow past a backward-facing step: A critical evaluation of two-equation models. AIAA Journal, 30(5):1314-1320, 1992.

[139] V.A. Titarev and E.F. Toro. Weno schemes based on upwind and centred tvd fluxes. Computers \& Fluids, 35:705-720, 2005.

[140] M.F. Tomé, A. Castelo, J.A. Cuminato, N. Mangiavacchi, and S. McKee. Gensmac3d: a numerical method for solving unsteady three-dimensional free surface flows. International Journal for Numerical Methods in Fluids, 37:747-796, 2001. 
[141] M.F. Tomé, A. Castelo, J. Murakami, J.A. Cuminato, R. Minghim, M.C.F. Oliveira, N. Mangiavacchi, and S. McKee. Numerical simulation of axisymmetric free surface flows. Journal of Computational Physics, 157:441-472, 2000.

[142] M.F. Tomé, N. Mangiavacchi, A. Castelo, J.A. Cuminato, and S. McKee. A finite difference technique for simulating unsteady viscoelástic free surface flows. Journal Non-Newtonian Fluid Mechanics, 106:61-106, 2002.

[143] M.F. Tomé and S. McKee. Gensmac: A computational marker-and-cell method for free surface flows in general domains. Journal of Computational Physics, 110:171-186, 1994.

[144] M.F. Tomé, S. McKee, L. Barratt, D.A. Jarvis, and A.J. Patrick. An experimental and numerical investigations of container filling with viscous liquids. International Journal for Numerical Methods in Fluids, 31:1333-1353, 1999.

[145] E.F. Toro. Riemann Solvers and Numerical methods for Fluid Dynamics, A practical introduction. 2nd Ed., Springer, 1991.

[146] H. Trac and H-L. Pen. A primer on eulerian computational fluid dynamics for astrophysics. Publication of the Astronomical Society of the Pacific, 115:303-321, 2003.

[147] E.R. van Driest. On turbulence flow near a wall. Journal of Aeronautical Sciences, 37:1007-1011, 1956.

[148] B. van Leer. Towards the ultimate conservative difference scheme ii. monotonicity and conservation combined in a second order scheme. Journal of Computational Physics, 361-370:14, 1974.

[149] A. Varonos and G. Bergeles. Development and assessament of a variable-order non-oscilatory scheme for convection term discretization. International Journal for $\mathrm{Nu}$ merical Methods in Fluids, 26:1-16, 1998.

[150] D. Violeau and R. Issa. Numerical modelling of complex turbulent free-surface flows with the sph method: an overview. International Journal for Numerical Methods in Fluids, 53:277-304, 2007.

[151] D.T Walker, R.I. Leighton, and L.O. Garza-Rios. Shear-free turbulence near a flat free surface. Journal of Fluid Mechanics, 320:19-51, 1996.

[152] D.C. Wan, B.S.V. Patnak, and G.W. Wei. Discret singula convolution-finite subdomain method for the solution of incompressible viscous flows. Journal of Computational Physics, 180:229-255, 2002. 
[153] R.F. Warming and R.M. Beam. Upwind second-order difference schemes and applications in aerodynamics. AIAA Journal, 4:12-41, 1976.

[154] N.P. Waterson and H. Deconinck. Design principles for bounded higher-order convection schemes - a unified approach. Journal of Computational Physics, 224:182-207, 2007.

[155] E.J. Watson. The radial spread of a liquid jet over a horizontal plane. Journal of Fluid Mechanics, 20:481-499, 1964.

[156] G.W. Wei and Y. Gu. Conjugated filter approach for solving burger's equation. Journal of Computational and Applied Mathematics, 149:439-456, 2002.

[157] J.J. Wei, B. Yu, W.Q. Tao, Y. Kawaguchi, and H.S. Wang. A new high-order-accurate and bounded scheme for incompressible flow. Numerical Heat Transfer, 43:19-41, 2003.

[158] F.M. White. Fluid Mechanics. McGRAW-HILL, 1979.

[159] D.C. Wilcox. Turbulence modeling for CFD. DCW Industries, Inc, California, 1993.

[160] V. Yakhot and S.A. Orszag. Renormalization group analysis of turbulence i. basic theory. Journal of Scientific Computing, 1:3-51, 1986.

[161] V. Yakhot, S.A. Orszag, S. Thangam, T.B. Gatski, and C.G. Speziale. Development of turbulence models for shear flows by a double expansion technique. The Physics of Fluids, 4:1510-1520, 1992.

[162] H. Yang. An artificial compression method for eno schemes: the slope modification method. Journal of Computational Physics, 89:125-160, 1990.

[163] Y.H. Zahran. Rcm-tvd hybrid scheme for hyperbolic conservation laws. International Journal for numerical Methods in Fluids, 57:745-750, 2008.

[164] X. Zhao, P.G. Richards, S.J. Zhang, and J. Liu. High-resolution schemes for bubbling flow computations. Applied Mathematical Modelling, 29:1232-1251, 2005.

[165] J. Zhu. On the higher-order bounded discretization schemes for finite volume computations of incompressible flows. Computer Methods in Applied Mechanics and Engineering, 98:345-360, 1992.

[166] D.W. Zingg, H. Lomax, and H. Jurgens. High accuracy finite difference schemes for linear wave propagation. SIAM J. Sci. Comput., 17:328-346, 1996. 